\title{
WestVirginiaUniversity
}

THE RESEARCH REPOSITORY @ WVU

Graduate Theses, Dissertations, and Problem Reports

2007

\section{Feasibility of using wearable devices for collecting pedestrian travel data}

Rohit Ajmera

West Virginia University

Follow this and additional works at: https://researchrepository.wvu.edu/etd

\section{Recommended Citation}

Ajmera, Rohit, "Feasibility of using wearable devices for collecting pedestrian travel data" (2007). Graduate Theses, Dissertations, and Problem Reports. 4284.

https://researchrepository.wvu.edu/etd/4284

This Thesis is protected by copyright and/or related rights. It has been brought to you by the The Research Repository @ WVU with permission from the rights-holder(s). You are free to use this Thesis in any way that is permitted by the copyright and related rights legislation that applies to your use. For other uses you must obtain permission from the rights-holder(s) directly, unless additional rights are indicated by a Creative Commons license in the record and/ or on the work itself. This Thesis has been accepted for inclusion in WVU Graduate Theses, Dissertations, and Problem Reports collection by an authorized administrator of The Research Repository @ WVU. For more information, please contact researchrepository@mail.wvu.edu. 


\title{
Feasibility of Using Wearable Devices for Collecting \\ Pedestrian Travel Data
}

\author{
Rohit Ajmera \\ Thesis submitted to the \\ College of Engineering and Mineral Resources \\ at West Virginia University \\ in partial fulfillment of the requirements \\ for the degree of \\ Master of Science \\ in \\ Civil Engineering
}

Ronald W. Eck, P.E., Ph.D., Chair

Darrell R. Dean, Jr., Ph.D.

Lloyd J. French, P.E., Ph.D.

Department of Civil and Environmental Engineering

Morgantown, West Virginia

2007

Keywords: Pedestrian Travel Data, Travel Diaries, Pedometer, GPS Devices, Hand-Held GPS Unit, Wearable GPS Data-Logger, Travel Log 


\section{ABSTRACT \\ Feasibility of Using Wearable Devices for Collecting \\ Pedestrian Travel Data}

\section{Rohit Ajmera}

Information on the travel characteristics of pedestrians is needed in the planning and design of pedestrian facilities. Desired information includes route selected, travel speed, trip origin and destination, and delay. Conventional methods of acquiring pedestrian travel data such as trip diaries suffer from a number of limitations.

Pedometers are simple wearable devices that are receiving considerable attention in the health promotion and physical activity fields. In recent years, there have been significant developments in global positioning system (GPS) technology. User-friendly devices are now available for under $\$ 100$. At the same time, more expensive wearable GPS data loggers are available in the market that are capable of collecting more extensive data. While the technology offers great potential in terms of data collection capabilities, questions about accuracy, reliability, user acceptability, and post-processing requirements must be addressed.

A formal assessment was conducted of pedometers, a hand-held GPS unit and a wearable data logger to determine their feasibility in collecting pedestrian travel data. Experiments were devised and conducted to assess the accuracy and reliability of the devices in a variety of conditions including heavy precipitation, dense vegetative cover and between tall buildings. In addition, devices were given to a number of subjects who used them outdoors for a 24-hour period. Each subject also completed a brief questionnaire intended to assess user acceptability of these devices. Results indicated that the pedometer is not suitable for collecting pedestrian travel data. The GPS devices hold promise as data collection devices as long as their limitations are taken into account. The paper presents recommendations about the suitability of each device for collecting pedestrian travel data. 


\section{ACKNOWLEDGEMENTS}

I wish to express my appreciation to all persons who have assisted, helped and advised in the completion of this report. Special thanks and recognition is extended to Dr. Ronald W. Eck, the Advisory Committee Chairman. His valuable suggestions, continued support and encouragement helped me in the completion of this project. I also extend my sincere gratitude to Dr. Darrell Dean and Dr. James French, my advisory committee members, for their assistance and suggestions. I am also grateful to Dr. Jim Rye for his help and input during the course of study. Grateful appreciation is also extended to the volunteer subjects who donated their time to assist me in meeting project objectives.

I would also like to thank my dear friend Sanket for the assistance he rendered in collecting data for this project. Lastly, I would like to thank my wife, Neha and my parents for their patience and support. 


\section{TABLE OF CONTENTS}

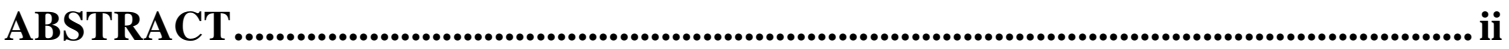

ACKNOWLEDGEMENTS ..................................................................................iii

TABLE OF CONTENTS ................................................................................... iv

LIST OF TABLES .............................................................................................................. vii

LIST OF FIGURES

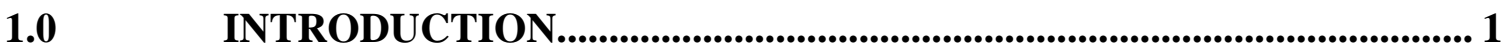

1.1 Background and Problem Statement ……........................................ 1

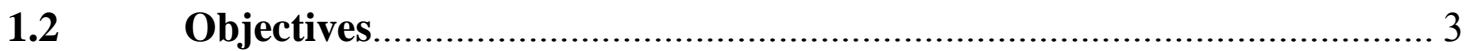

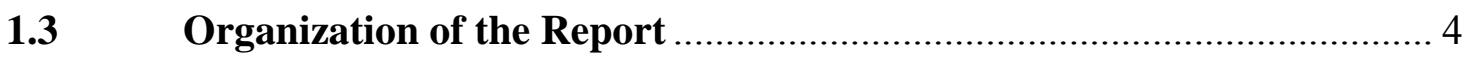

2.0 REVIEW OF LITERATURE .............................................................. 5

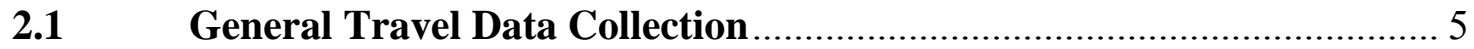

2.1.1 Counting Devices (Motorized means of transport)........................................ 7

2.1.2 Traffic Stream Characteristics.............................................................. 9

2.1.3 Travel Diaries ...................................................................................... 11

2.1.4 Other Methods of Collecting Travel Data ………................................... 17

2.2 Pedestrian / Bicycle Travel Data ……………...................................... 19

2.2.1 Data on Usage, Trip and User Characteristics ......................................... 20

2.3 Conventional Methods of Data Collection............................................ 24

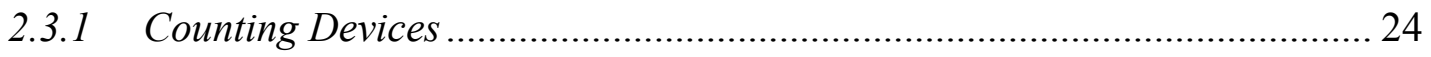

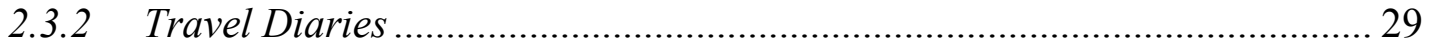

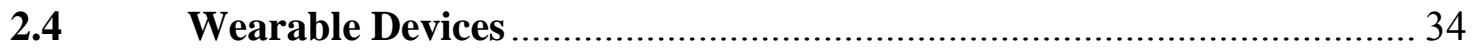

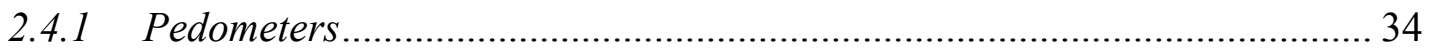

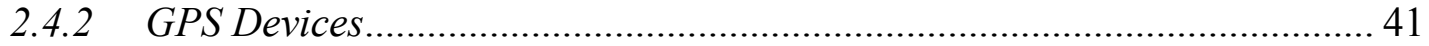

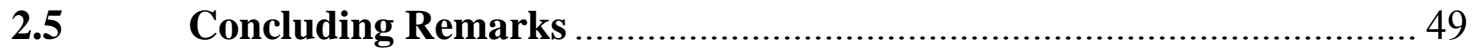

3.0 METHODOLOGY ................................................................................ 51

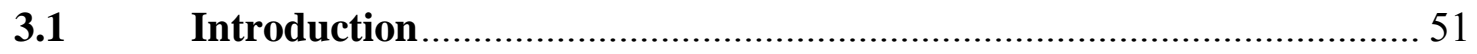

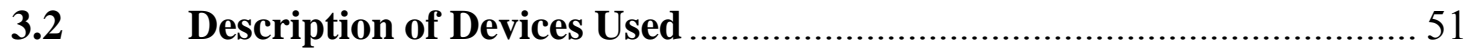

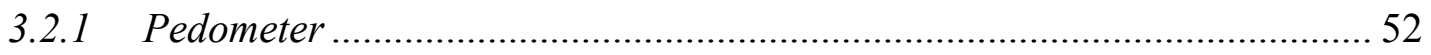

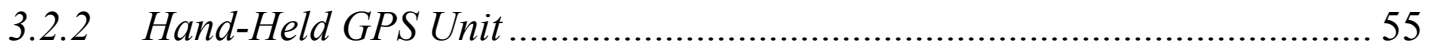

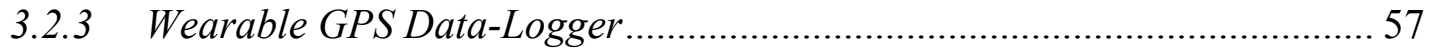


3.3 Evaluation of Device Characteristics 59

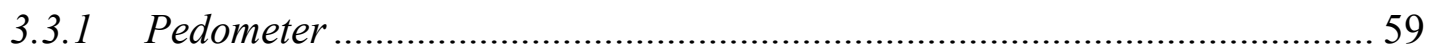

3.3.1.1 Effect of Surface Type on Number of Steps ……………………........ 62

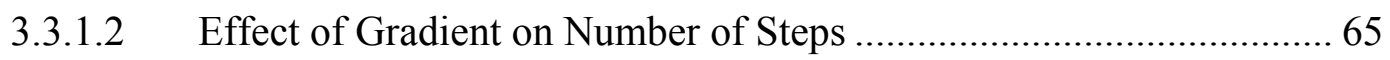

3.3.1.3 Effect of Social Interaction on Number of Steps ................................ 67

3.3.1.4 Effect of Other Pedestrian Traffic on Number of Steps ...................... 69

3.3.2 Hand-Held GPS Unit and Wearable GPS Data-Logger ............................ 74

3.3.2.1 Signal Availability Under High-Voltage Power Lines and Towers ... 76

3.3.2.2 Signal Availability Under Dense Tree Canopy ……………………... 78

3.3.2.3 Signal Availability in Heavy Rain ................................................... 80

3.3.2.4 Signal Availability in the Vicinity of Tall Buildings.......................... 82

3.4 Evaluation of Operational Considerations ………………………......... 85

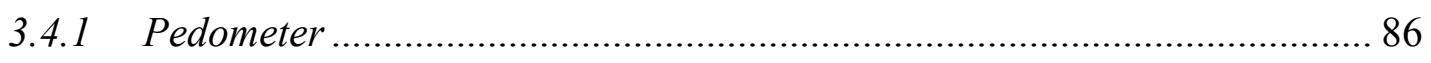

3.4.1.1 Use of a Pedometer for Collecting Non-Motorized Travel Data........ 88

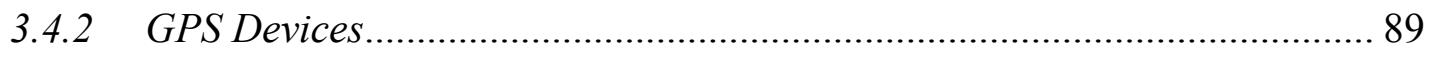

3.4.2.1 Use of Hand-Held GPS Device for Collecting Non-Motorized Travel

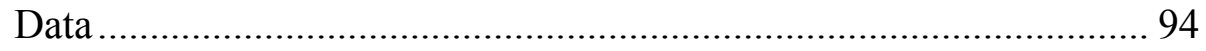

3.4.2.2 Use of Wearable GPS Data-Logger Unit for Collecting Non-Motorized

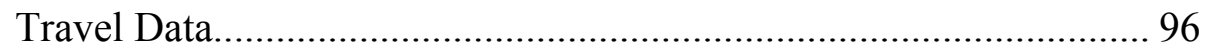

4.0 DATA ANALYSIS AND RESULTS .................................................... 98

4.1 Evaluation of Device Characteristics - Pedometers ............................... 98

4.1.1 Effect of Surface Type on Number of Steps............................................... 98

4.1.2 Effect of Gradient on Number of Steps .................................................. 103

4.1.3 Effect of Social Interaction on Number of Steps ........................................ 108

4.1.4 Effect of Other Pedestrian Traffic on Number of Steps ............................ 113

4.2 Evaluation of Device Characteristics - Hand-Held GPS Unit and

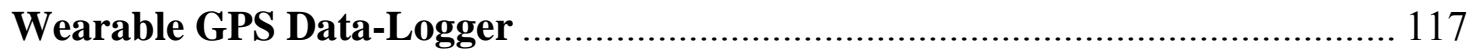

4.2.1 Signal Availability Under High-Voltage Power Lines and Transmission

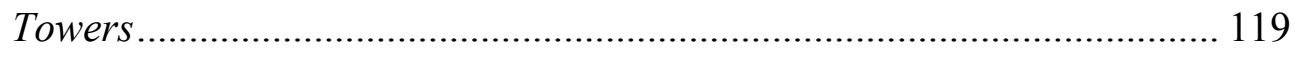

4.2.2 Signal Availability Under Dense Tree Canopy........................................ 122

4.2.3 Signal Availability in Heavy Rain .............................................................. 126 
4.2.4 Signal Availability in the Vicinity of Tall Buildings .................................. 128

4.3 Evaluation of Operational Considerations - Pedometer …………….... 137

4.4 Evaluation of Operational Considerations - Hand-Held GPS Unit .... 145

4.5 Evaluation of Operational Considerations - Wearable GPS Data-Logger

5.0 CONCLUSIONS AND RECOMMENDATIONS................................ 181

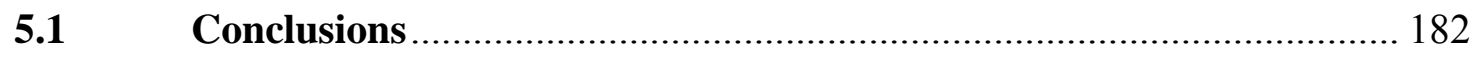

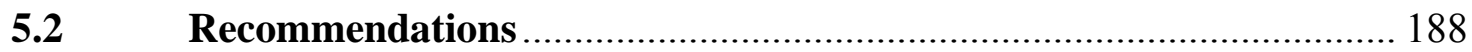

LIST OF REFERENCES..................................................................................... 193

APPENDIX A - Table of the Standard Normal (z) Distribution................................. 196

APPENDIX B - Instruction Sheets and Data Collection Forms for Experiments to Evaluate Device Characteristics ....................................................... 198

APPENDIX C - Instruction Sheets and Data Collection Forms for Experiments to

Evaluate Operational Considerations .................................................... 215 


\section{LIST OF TABLES}

Table 1 - Pedestrian LOS Criteria as Given by Highway Capacity Manual (TRB 2000). 74

Table 2 - Characteristics of Subjects in the Effect of Surface Type on Number of Steps

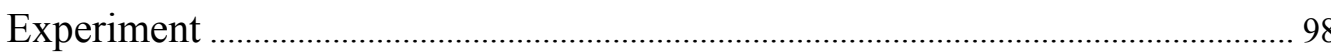

Table 3 - Average Number of Steps for Each Surface Type................................................... 99

Table 4 - P-Values Obtained from Comparison of Steps on Different Surfaces................. 102

Table 5 - Characteristics of Subjects in the Effect of Gradient on Number of Steps

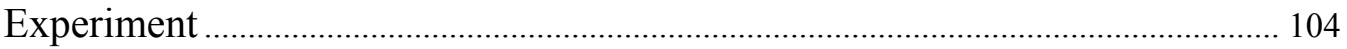

Table 6 - Data for Each Gradient Class Collected From 12 Subjects ................................... 105

Table 7 - P-Values Obtained from Comparison of Steps on Different Gradients ............. 107

Table 8 - Characteristics of Subjects in the Effect of Social Interaction on Number of

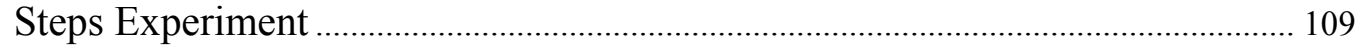

Table 9 - Number of Steps Required by Researcher for Different Levels of Social

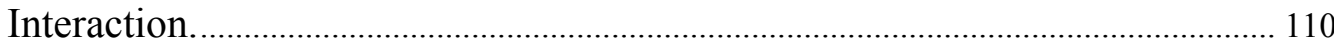

Table 10 - P-Values Obtained from Comparison of Steps for Different Levels of Social Interaction.

Table 11 - Data Obtained After Analysis of All Test Runs for Effect of Other Pedestrian Traffic on Number of Steps 114

Table 12 - Description of Various Fields in the Tabular Data Obtained from the HandHeld GPS Unit

Table 13 - Description of Various Fields in the Tabular Data Obtained from the Wearable GPS Data-Logger

Table 14 - Recorded Data from one Experiment Using the Hand-Held GPS Device ..... 118

Table 15 - Recorded Data from one Experiment Using the Wearable GPS Data-Logger

Table 16 - Sample Data Obtained from Hand-Held GPS Unit for Effect of High-Voltage Power Lines and Transmission Towers on Availability of Satellite Signal... 121

Table 17 - Sample Data Obtained from Wearable GPS Data-Logger Unit for Effect of High-Voltage Power Lines and Transmission Towers on Availability of Satellite Signal 
Table 18 - Sample Data Obtained from Hand-held GPS Unit for Effect of Dense Tree Canopy on Availability of Satellite Signal

Table 19 - Sample Data Obtained from Wearable GPS Data-Logger Unit for Effect of Dense Tree Canopy on Availability of Satellite Signal

Table 20 - Data Obtained from Hand-Held GPS Unit for Effect of Heavy Rain on Availability of Satellite Signal Experiment

Table 21 - Data Obtained from Wearable GPS Data-Logger Unit for Effect of Heavy

Rain on Availability of Satellite Signal Experiment.

Table 22 - Data Obtained from Hand-held GPS Unit at Site 1, for Effect of Tall Buildings on Availability of Satellite Signal Experiment.

Table 23 - Data Obtained from Wearable GPS unit at Site 1 for Effect of Tall Buildings on Availability of Satellite Signal Experiment.

Table 24 - Data from Recording Sheet at Site 2 for Effect of Tall Buildings on Availability of Satellite Signal Experiment 131

Table 25 - Data Obtained from Hand-Held GPS Unit at Site 2 for Effect of Tall Buildings on Availability of Satellite Signal Experiment.

Table 26 - Data Obtained from Wearable GPS Unit at Site 2 for Effect of Tall Buildings on Availability of Satellite Signal Experiment

Table 27 - Data Obtained from Hand-held GPS Unit at Site 3 for Effect of Tall Buildings on Availability of Satellite Signal Experiment. 133

Table 28 - Data Obtained from Wearable GPS Unit at Site 3 for Effect of Tall Buildings on Availability of Satellite Signal Experiment.

Table 29 - Summary of Trip Information Obtained from Subjects in Pedometer

Experiment

Table 30 - Data Recorded by Subject 4 in the Travel Log Form for Pedometer

Experiment .....

Table 31 - Summary of Subject Responses to Questionnaire on Pedometer Study 143

Table 32 - Summary of Walking Trip Information Obtained from Subjects in Hand-Held GPS Unit Experiment. 146

Table 33 - Typical Data Recorded by Subject lin the Travel Log Form in Hand-Held GPS Experiment 
Table 34 - Sample Data Obtained from Hand-Held GPS Unit for Trip 1

Table 35 - Sample Data Obtained from Hand-Held GPS unit for Trip 2 156

Table 36 - Summary of Subject Responses to Questionnaire on Hand-Held GPS Device

Table 37 - Summary of All Walking Trips for All Subjects Obtained Using a Wearable GPS Data-Logger 165

Table 38 - Typical Data Recorded by Subject 1in the Travel Log Form in Wearable GPS Data-Logger Experiment 166

Table 39 - Sample Data Obtained from Wearable GPS Data-Logger Unit for Trip 1 for Subject 1

Table 40 - Sample Data Obtained from Wearable GPS Data-Logger Unit for Trip 2 for Subject 1 . 173

Table 41 - Summary of Subject Responses to Questionnaire on Wearable GPS DataLogger. 


\section{LIST OF FIGURES}

Figure 1: Sample Travel Diary from San Francisco Bay Area (1990) (Axhausen, 1995) 14

Figure 2: Sample Travel Diary from: Dallas/Fort Worth (1995) (Axhausen, 1995) ......... 15

Figure 3: Yamax Digi-Walker Pedometer Used in This Study ................................................ 54

Figure 4: (a) ETrex Hand-Held GPS Device and; (b) Holster Used for This Study ........... 56

Figure 5: Close-up View of Display Screen of Etrex Hand-held GPS Device in Upright

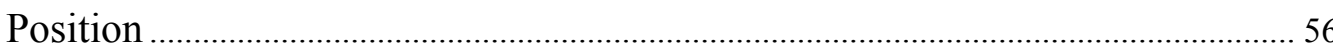

Figure 6: Components of the GPS Data Logger Used in This Study .................................... 58

Figure 7: Wearable GPS Data-Logger Worn by a User. Note That the Antenna is

Positioned on the Shoulder.

Figure 8: Standard Normal Distribution Showing the Critical Value z $\alpha$ /2 in the Right

Tail.

Figure 9: Illustrations of the Types of Surfaces used in the Pedometer study. 63

Figure 10: Various Gradient Categories Used in the Pedometer Study. 66

Figure 11: Photo of Site 1, from Observer's Vantage Point, Used to Calculate Level of Service 72

Figure 12: Photo of Site Used for Effect of High-Voltage Power Lines and Transmission Towers on Signal Availability Experiment 76

Figure 13: Sites Used for Effect of Dense Tree Canopy on Signal Availability

Experiment 79

Figure 14: WVU Campus Location Used for Effect of Tall Buildings on Availability of Satellite Signal

Figure 15: Downtown Morgantown Location Used for Effect of Tall Buildings on Availability of Satellite Signal 83

Figure 16: Downtown Pittsburgh Location, Used for Effect of Tall Buildings on Availability of Satellite Signal 84

Figure 17: Average Number of Steps By Surface Type. 100

Figure 18: Standard Deviation of Steps By Surface Type 101

Figure 19: Average Number of Steps Required for Each Gradient Class. 105

Figure 20: Standard Deviation of Steps for Each Gradient Class. 106 
Figure 21: GPS Data for First Trip Analyzed Obtained Using Hand-Held GPS Device, Plotted on Arcview GIS Software 149

Figure 22: Plot of Elevation Versus Time for Trip 1, Obtained from Hand-Held GPS Unit 151

Figure 23: Speed Profile for Trip 1 Obtained from Hand-Held GPS Device Experiment 152

Figure 24: GPS Data for Second Trip Analyzed for Subject 3 Obtained Using Hand-Held GPS Device, Plotted on Arcview GIS Software. 154

Figure 25: Plot of Elevation Versus Time for Trip 2, Obtained from Hand-Held GPS Unit 157

Figure 26: Speed Profile for Trip 2 Obtained from Hand-Held GPS Device Experiment

Figure 27: GPS Data for Two Trips in the Vicinity of the WVU Campus for Subject 1, Obtained Using Wearable GPS Data-Logger and Plotted on Arcview GIS Software. 168

Figure 28: Profile for Trip 1 Obtained from Wearable GPS Data-Logger 169

Figure 29: Speed Profile for Trip 1 Obtained from Wearable GPS Data-Logger ... 170

Figure 30: Profile for Trip 2 Obtained from Wearable GPS Data-Logger .. 174

Figure 31: Speed Profile for Trip 2 Obtained from Wearable GPS Data-Logger 175 
Chapter 1

\subsection{INTRODUCTION}

\subsection{Background and Problem Statement}

Walking is the most basic form of transportation. Until recently, walking and bicycling have not been considered as viable transportation solutions. However, there has been a growing interest in walking as a mode of transport and/or for recreation due to an increase in the concern for public health and as a result of problems associated with motorized means of travel. The potential of pedestrian and bicycle travel to provide mobility, reduce congestion, improve environmental quality, and promote public health has received increasing attention in recent years. Walking is being realized as a key ingredient to livable communities. It provides a means by which to connect the various activities of a neighborhood, without the need for auto trips, and is almost always a segment of each public transit trip and often a segment of an auto trip. Walking is expected to grow in popularity as public recognizes the benefits of walking and as more pedestrian friendly designs are implemented. There is now considerable interest in designing new facilities for, and adapting existing infrastructure to, non-motorized travel.

Methods of collecting travel data (such as volume, speed and travel time) for motorized modes are well-established. However, planners and engineers have tended to undercount non-motorized travel by ignoring short trips, non-work travel, travel by children and recreational travel. These short trips account for a majority of the trips made by non-motorized means and thus travel data on these trips should play a role in facility planning, design and construction. 
Information on the travel characteristics of and facility usage data by pedestrians is needed in the planning and designing of pedestrian facilities. Needed information includes data on route selected, travel speeds, trip origin and destination and pedestrian volumes. The conventional methods for pedestrian data collection include trip diaries and trip logs, which although simple, may burden the user and tend to show error. Error may include under counts, incomplete counts, improper logging of times and other human errors of this nature. Error may also be due to traffic conditions such as standing at an intersection waiting for the walk signal that may increase the travel time, or increase in trip times due to a gradient in the route.

A basic method of acquiring pedestrian count data is through manual counts. Since the technique is labor-intensive, this is a costly method, particularly if done on a large scale. Consequently, a variety of new technology is being applied to pedestrian detection and counting, including Infrared Detectors, Microwave Detectors, Heat Sensors and Laser Counters. However, these techniques have their own limitations. When pedestrian traffic becomes heavy, the detection of individuals becomes complicated. There can be error in observations during heavy rains and other severe weather conditions. Also, pedestrians outside the detection zone can be missed. Since they essentially monitor pedestrian activity at a point, these techniques do not provide information concerning trip length or travel times, which may be of considerable importance for both planning and facility design. Also, as the technology gets more sophisticated for these devices, the cost of buying, installing and maintaining them continues to increase, which may preclude their use in large scale projects. 
New technology is not limited to fixed sensors. There are presently various data collection devices that can be worn by individuals and, thus, have the potential to provide data on pedestrian travel behavior. In the physical activity area, there is considerable interest in pedometers as a motivational technique to encourage walking since they allow users to monitor and measure, in a simple fashion (using steps), their physical activity. Given the low cost and popular appeal, can the pedometer be used to collect certain pedestrian travel data?

In recent years, there have been considerable developments in Global Positioning System (GPS) technology. User-friendly devices are now available for under $\$ 100$. These units can provide information on path of travel and possibly travel time. At the same time, there are more expensive wearable GPS data loggers on the market. These devices can keep track of time, location and altitude for up to three days on a single battery charge. While this offers great potential in terms of data collection capabilities, questions about cost, user acceptability, device limitations and post-processing requirements must be addressed. For all of the devices mentioned, accuracy is an issue. Therefore, it seems appropriate to conduct a formal assessment of these wearable devices to determine the feasibility of their use in collecting pedestrian travel data.

\subsection{Objectives}

This study was conducted to assess the feasibility of using various wearable devices in collecting pedestrian travel data. The overall purpose of this project was to determine the feasibility of using wearable devices namely pedometers, GPS data loggers and hand-held GPS units, for acquiring pedestrian travel data. In order to accomplish the overall goal, several specific objectives were established: 
- To conduct a thorough review of the literature on various methods for collecting pedestrian travel data including conventional techniques and those using new technology.

- To identify and characterize different wearable data collection devices and acquire several of these for testing purposes.

- To develop an experimental plan to assess the feasibility of using wearable devices to collect pedestrian travel data.

- To carry out the experimental plan through laboratory and field testing.

- To analyze the collected data.

- To develop conclusions about the suitability of each device and recommendations for their use in collecting pedestrian travel data.

- To document the work in the form of a thesis.

\subsection{Organization of the Report}

This report consists of five chapters. Chapter 1 has presented background material, identified the problem and outlined the project objectives. Chapter 2 is a critical review of the relevant literature, including general data collection methods for both motorized and non-motorized travel and descriptions of new technology for pedestrian data collection. Chapter 3 describes the experimental plan and the methodology used in conducting the feasibility study. Chapter 4 presents an analysis of the data collected and describes project results. Chapter 5 presents conclusions about the feasibility of each device. Recommendations for their use in collecting pedestrian travel data are included along with recommendations for follow-on research. 


\section{Chapter 2}

\subsection{REVIEW OF LITERATURE}

This chapter summarizes and critically reviews previous research that has been conducted relative to data collection for both motorized and non-motorized means of transportation. It includes various devices and techniques that have been used to date to collect travel data used in planning and designing of transportation facilities. Since this project was primarily concerned with assessing the feasibility of using wearable devices for collecting pedestrian travel data, the review of literature will focus on that topic. However, in order to put the problem in proper context, the literature review covers the topic of travel data collection generally, including that for motorized means, even though the emphasis is on conventional and contemporary methods of collecting pedestrian data. The latter part of the review discusses the literature dealing with wearable devices, i.e., pedometers, hand-held GPS units and wearable data loggers, and reviews their characteristics and applications for obtaining travel data.

\subsection{General Travel Data Collection}

Travel data collected for planning generally or for specific use in a transportation project can play an important role in improving the transportation system. Travel data in its simplest form consists of information on the performance of the existing transportation system or facility, and can help to predict performance in the future. Transportation planning largely depends on the quality of travel data that is used as an input at various levels of planning processes. The existing transportation system requires that a variety of data and information be assembled which adequately describe the current status of 
systems, facilities and traffic. For any transportation project to be successful in the long run, information or data has to be collected which helps not only in planning and designing but also for analyzing various situations. Data are collected for many reasons and applications (Roess, et al; 2004):

- Investigating trends over time - traffic engineers need trend data to help forecast future transportation needs.

- Managing the physical system - inventories of physical elements are always needed. These inventories help assess which items need to be replaced or repaired, and on what anticipated schedule.

- Understanding the needs and choices of the public and industry -this data is required for planning and development purposes to get a good measure of how and why people travel.

- Assessing the effectiveness of improvements - when improvements of any kind are implemented, follow-up studies are needed to confirm their effectiveness and to allow for adjustments if all objectives are not fully met

- Assessing potential impacts - an essential part of traffic engineering is the ability to predict and analyze projected traffic impacts of new developments with the help of data collected and to provide traffic input to air pollution models.

- Evaluating facility or system performance - all traffic facilities and systems must be periodically studied to determine whether they are delivering the intended quantity and quality of access and/or mobility service to the public.

- Calibrating basic relationships or parameters - fundamental measures, such as perception-reaction time, discharge headways at a signalized intersection, headway 
and spacing relationships on freeways and other uninterrupted flow facilities, and other key parameters and relationships must be properly quantified and calibrated to existing conditions.

Transportation data collection and traffic studies provide the underpinning of all traffic planning, design and analysis. If the data is incomplete (e.g. limited data on nonmotorized modes) or not accurate, then the engineering that is based on it may be flawed. Some of the tasks involved in data collection, reduction, analysis and presentation are somewhat mundane. Planners use a variety of methods for collecting the data needed for input into the planning process. The type of data gathered varies with the type of facility that is being studied and also with the purpose of study. The data collected can be either travel data or performance data.

The most basic measurement in collecting travel data is counting--counting vehicles, passengers, pedestrians or bicyclists. These count measures are used to produce estimates of volumes, demand and quality of flow, which are in turn helpful in the planning and design of various transportation facilities. Performance data may include information on speeds, travel-times, route selected, origins/destinations and arrival/departure times. These data are gathered using various techniques, methods and devices.

\subsubsection{Counting Devices (Motorized means of transport)}

Manual data collection by direct observation in the field, with use of standard paper forms, was the most prevalent method for collecting travel data (such as turning movement counts) for many years. Mechanical hand counters have also been in wide use for decades to count the number of vehicles which pass a point on the road. Buttons are 
depressed to advance interlocking mechanical wheels. Different buttons are used for specific movements (left, through, right), for classification of vehicles or for other purposes.

In-road mechanical traffic counters, activated by a pressure tube (pneumatic) as wheels cross the tube, have been the mainstay of many traffic counting programs when extended counts are needed but a more permanent installation is not economically justified. Wire loops buried in the pavement have long served both traffic counting and traffic control purposes. These loop detectors are not used in locations where there is frequent pavement construction, special disruptions, or frost heave of the pavement. In such places, radar, ultrasonic and magnetic detectors can be used to detect presence or passage of vehicles (Roess, et al; 2004). Some volume counting detectors are so small and low-powered that they are literally nailed down in the pavement, and removed later. Some detectors transmit their data to a remote station by wireless communication. Mechanical traffic counters are economical in cost in comparison with the radar, ultrasonic and pressure plate detectors, which can be expensive. The type of counters used for counts depends on various factors such as data sought, costs and environmental factors.

Modern techniques of data collection are now being used to collect data more easily and accurately. Video images are now being captured and digitized so that software algorithms can identify not only volumes, but vehicle classification and speeds. Virtual detectors are being placed at intersections and other locations by software linked to video, so that the function of many loop detectors is accomplished. 


\subsubsection{Traffic Stream Characteristics}

Other types of traffic engineering studies try to quantify the characteristics of the traffic stream, e.g. travel time and delay studies. A travel time study determines the amount of time required to travel from one point to another on a given route. In conducting such a study, information may also be collected on the locations, durations and causes of delay as well as average speeds on individual links. The collection of travel time and delay data helps in giving an indication of the level of service of the study section. These data also aid the traffic engineer in identifying specific problem locations, which may require special attention in order to improve the overall flow of traffic on that route. The data obtained from travel time and delay studies may also be used for:

- Determining the efficiency of a route with respect to its ability to carry traffic.

- Identifying locations with relatively long delays and the causes for those delays.

- Performing before and after studies to evaluate traffic operation alternatives that reduce travel time.

- Performance of economic studies in the evaluation of traffic operation alternatives that reduce travel time.

There are a number of methods used to conduct travel time and delay studies. These can be grouped into those that require a test vehicle and the ones that do not require a test vehicle. The methods that do not require a test vehicle for collecting travel time and delay are License-Plate method, Interview method and Postcard studies. The category that requires a test car involves two principal techniques:

- Test car methods (Garber and Hoel, 2002; Roess, et al; 2004) - Under this method, the test car is driven by an observer along the test section so that the test car moves in 
traffic. The test car method requires two to three observers including the driver, equipped with suitable recording material such as pencil and paper or a laptop computer. Various records along the test route, such as intermediate points, times, speeds, delay times, causes of delay, and time to traverse the test section are kept. The test car method can be subdivided into three possible variations - the FloatingCar technique, the Maximum Car technique and Average Car technique. These variations are different only in the way the test car is driven on the test route. All these techniques give the travel time and sources and duration of delays along a route.

- Moving-Vehicle technique (Garber and Hoel, 2002) - In this technique, as explained by Garber and Hoel, (2002), the observer makes a round trip on a test section, where it is assumed that the road runs east-west. The observer starts collecting the data at the starting point, as the driver takes the car eastward to the end point, and then turns around and drives westward to the starting point again. As the test vehicle makes the round trip, not only are the time of identified check points recorded in each direction, but also the number of vehicles traveling west in the opposite lane while the test car is traveling east; the number of vehicles traveling westbound that overtake the test car while it is traveling west, and; the number of vehicles traveling westbound that the test car passes while it is traveling westbound. In this way, not only the average travel time but also the volume in the westbound direction and subsequently the volume in the eastbound direction can be obtained. The volume in both directions can be obtained using the expression given by Garber and Hoel, (2002). The moving vehicle method also provides an estimate of space mean speed. This method is 
different from other methods of collecting data as it estimates the volumes in both directions simultaneously, in addition to collecting travel times.

\subsubsection{Travel Diaries}

Transportation planners use travel demand models to estimate current trends and changes in transportation activity over time. Travel survey, or travel diary methods are used to collect the input and calibration data (origins/destinations) used to derive and validate travel demand models. The travel diary, according to Axhausen (1995), is a survey instrument designed to record trips of a person over a given period of time with relevant details for that particular time period. Information from travel diaries is a central data source for understanding and measuring the travel behavior of individuals and households and, therefore, is essential to the comprehensive planning and monitoring of transport policy, operations and infrastructure.

Basic demand or usage indicators may be developed from either systematic counts or regular travel surveys. Surveys have the potential for tracking usage by characteristics of the user, trip purpose, and origin/destination. In addition to these data, information on mode of transport used, as well as the departure and arrival times can also be recorded. Travel surveys are usually carried out or conducted using paper-and- pencil interview methods in the form of mail-out-mail-back surveys with in-home interviews. These mail-out travel diary survey forms are prepared before-hand and include questions, which are relevant to the type of study that is being carried out. Most of the travel surveys at the national and local level are carried out by providing travel survey forms to a small sample of the population of interest. 
This technique can be used to collect data for all modes of transportation, both motorized and non-motorized. Collecting diary entries, as a means of gathering trip information, has been used by transportation planners for many years. The devices used to make diary entries have evolved from traditional pencil and paper forms to state-ofthe-art GPS-connected Personal Digital Assistant (PDAs). According to Axhausen (1995), the travel diary consists of:

- The recording of all trips sequentially during a specific period of time.

- The instrument records person-specific information for a household.

- The household instrument records relevant household-based information.

- The resource instrument recording the relevant details about the physical and social means available to the household. Examples are cars and other motor vehicles available to household, bicycles, access to public transport and telephones.

The content of a specific travel diary, according to Axhausen (1995), is a compromise between many interests, which have to be accommodated within the survey budget of the sponsor and within the time which the respondents are willing to give to the survey. The data items required from the travel diary vary with the project and address questions relevant to the purpose of the project. Rather than focusing the research on one type of trip or on one source of information, diary entries encompass all travel modes, trip purposes and information media - in the proportions that they were actually employed. In as much as the diary entries are records of actual trips taken under realworld conditions and constraints, they provide a valuable source of data to complement the existing stated preference and simulation research. The entries in the travel diaries regarding access to private vehicle or nearness to public transport provide valuable 
insight into the ways that traveler information is used to plan and modify trips, providing a new perspective to the knowledge in this field.

Sample travel diary forms are shown in Figures 1 and 2. The survey form in Figure 1 is a simple form which allows the respondents to record essential details during their journey. The survey form in Figure 2 requires more detailed recording of the trip activities by the respondent.

According to Peirce and Lappin, (April 2003), it should also be noted that a few days of diary entry records can provide only an idea of the respondent's travel, and may not be fully representative of travel behavior. Also, the additional respondent burden associated with filling out the survey might result in underreporting of information as some individuals elect not to participate (respond). Moreover, the extent to which findings from a particular area can be applied to other places and locations, particularly those with different transportation networks and geographic conditions, is unclear. Underreporting may also be evident in some cases, for example, in walking, where respondents may not record short walking trips out of a belief that these are not 'real' trips. Thus, the technique may result in an undercounting of non-motorized trips. Also, since this technique of data collection is primarily used for planning purposes, its use is typically restricted to urban areas, and facilities in these areas.

According to Transportation Research Board - Special Report 277 (2004), travel surveys often involve a two-stage process: a recruitment interview to obtain information about the household, followed by an extended data gathering interview to collect information on household travel. The 2001 National Household Travel Survey (NHTS) 
is an example of such a two-stage survey and includes the USDOT and FHWA

administered National Personal Travel Survey, carried out every five to seven years.

\section{MTC AREA TRANSPORTATION STUDY YOUR TRIP RECORD FOR ONE DAY}

Picase carry this trip card with you and write down each trip you make on TRAVEL DAY.

When you go from one place to another for any teason, this is a trip.

When you change your travel mode (car, bus, BART, bicycle, wailk, etc.) this is a new trip.

Picasc record an address if possible, or an easily identifiable location, where each trip begins and ends.

as well as the times for each trip beginning and ending.

PERSON:

\begin{tabular}{|c|c|c|c|c|c|c|c|}
\hline \multicolumn{2}{|r|}{ TRIP BEGINNING } & \multicolumn{2}{|c|}{ TRIP DESTINATION } & \multirow{2}{*}{$\begin{array}{l}\text { OESTINTIOIOE } \\
\text { PUAPOSE }\end{array}$} & \multirow{2}{*}{ 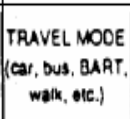 } & \multirow{2}{*}{ 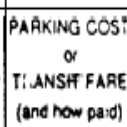 } & \multirow{2}{*}{$\begin{array}{l}\text { NUM } \\
\text { IN } \\
\text { CAF }\end{array}$} \\
\hline $\begin{array}{l}\text { START } \\
\text { TMME }\end{array}$ & ADDRESS AND CITY & ADDRESS AND CITY & $\begin{array}{l}\text { END } \\
\text { TIME }\end{array}$ & & & & \\
\hline & & & & & & & \\
\hline & & & & & & & \\
\hline & & & & & & & \\
\hline & & & & & & & \\
\hline & & case turm card oper and contin & & & & & \\
\hline
\end{tabular}

\begin{tabular}{|c|c|c|c|c|c|c|c|}
\hline \multicolumn{2}{|r|}{ TRIP BEGINNING } & \multicolumn{2}{|c|}{ TRIP DESTINATION } & \multirow{2}{*}{$\begin{array}{l}\text { DESTMUTION } \\
\text { PUPPOSE }\end{array}$} & \multirow{2}{*}{$\begin{array}{l}\text { TRAVEL MOOE } \\
\text { (CW, OU, BART, } \\
\text { 'walk, OtC.) }\end{array}$} & \multirow{2}{*}{$\begin{array}{l}\text { PARKING COST } \\
\text { TRUNSII FARE } \\
\text { (and how paid) }\end{array}$} & \multirow{2}{*}{$\begin{array}{c}\text { NUM } \\
\text { IN } \\
\text { CAF }\end{array}$} \\
\hline $\begin{array}{l}\text { STAAT } \\
\text { TIME }\end{array}$ & ADDRESS AND CITY & ADORESS AND CITY & $\begin{array}{l}\text { END } \\
\text { TIME }\end{array}$ & & & & \\
\hline & & & & & & & \\
\hline & & & & & & & \\
\hline & & & & & & & \\
\hline & & & & & & & \\
\hline & & & & & & & \\
\hline & & & & & & & \\
\hline & & & & & & & \\
\hline & & & & & & & \\
\hline & All information & confidential and used for tro & dlannin & urposes only & & & \\
\hline
\end{tabular}

Figure 1: Sample Travel Diary from San Francisco Bay Area (1990) (Axhausen, 1995) 

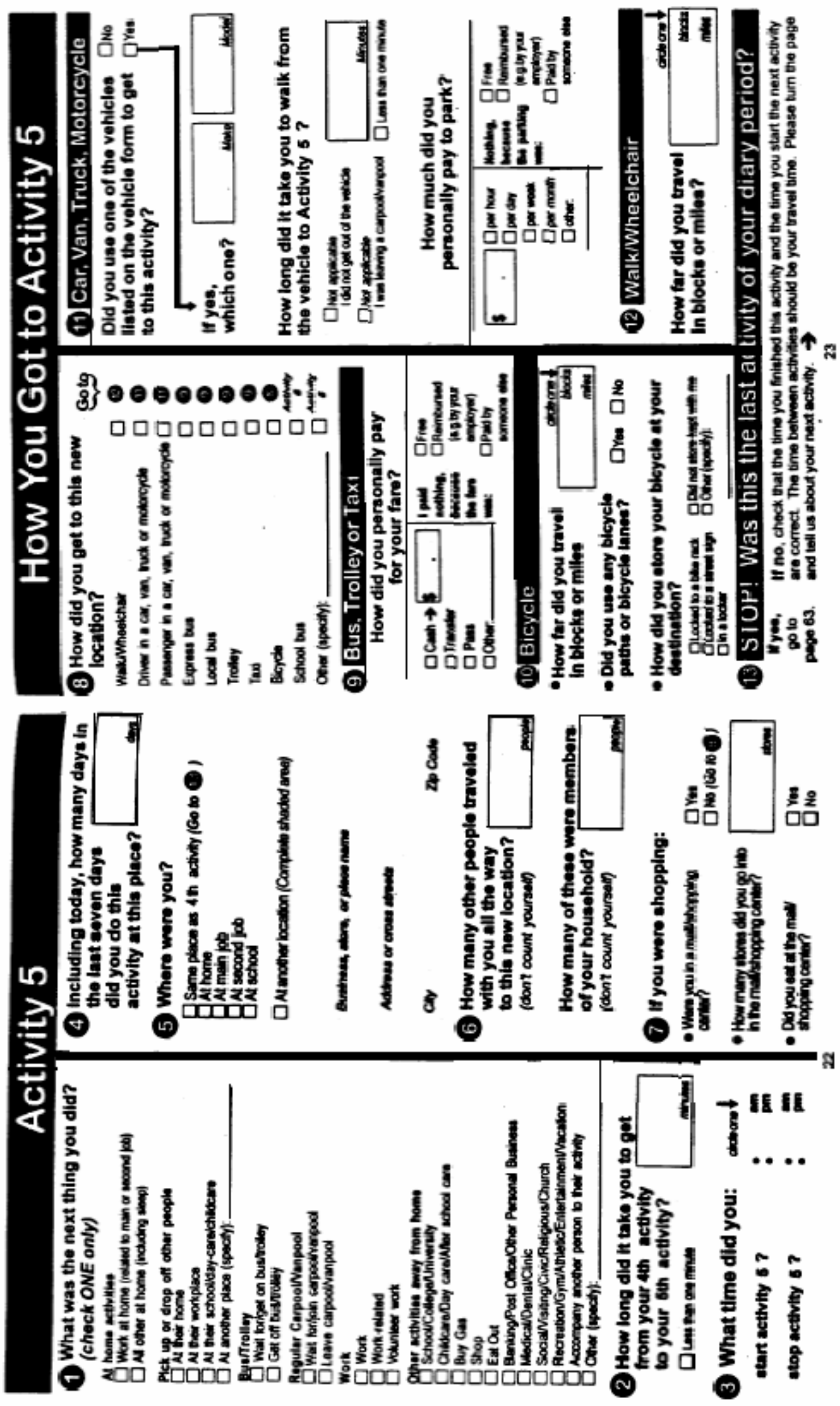

Figure 2: Sample Travel Diary from Dallas/Fort Worth (1995) (Axhausen, 1995) 
Since each interview has an accompanying level of non-response, the overall response rate - given by the product of the response rates for the two interviews - tends to be lower than that for a survey involving only one interview. The Bureau of Transportation Statistics is not alone in needing to develop more effective survey methods. More effective survey responses are also required by transportation planners for design purposes. Many of the methodological issues the agency faces (including the decline in the effectiveness of telephone surveys, the search for more cost-effective ways of collecting quality data, and the need to expand the availability of survey data without compromising the confidentiality of data providers) are common to the wider survey community.

The Transportation Research Board (2004) also states that non-response is major concern because of the potential for bias and the resulting implications for data quality. If the travel behavior of the non-respondents is not significantly different from that of the respondents, there may be no significant bias. However, there is a reason to believe that the travel patterns of survey non-respondents are significantly different from those of the respondents. Thus, the 2001 NHTS, which relies on telephone contacts for data collection, may give too much weight to the travel patterns of those who do not travel much and too little weight to the travel of more mobile groups in the society - a serious deficiency for a personal travel survey. The under-representation of certain socioeconomic groups and certain age groups, may also introduce bias into the travel survey. 


\subsubsection{Other Methods of Collecting Travel Data}

Rapid advancement of information technology has greatly enhanced the ability to collect, summarize, and analyze information. Technologies such as wide area sensing, image identification processes, wireless communications, satellite-based location systems, mapping technologies, and the like continue to revolutionize both the quantity and quality of data that can be collected, stored in recoverable formats, and analyzed. Some of the most modern technologies such as Tag system for automated toll collection can be used to track vehicle movements throughout the system by placing high-speed sensors at periodic intervals along major highways (Roess, et al., 2004). The potential to gain insight into travel characteristics and origin-destination patterns is immense.

Global positioning systems (GPS) can now be used to track vehicle fleets, such as trucks and rail cars. They can also be used as individual vehicle guidance systems, now available on most cars (Roess, et al., 2004). Urban applications of GPS mapping systems include mapping the transportation and facility infrastructure. Streets and highways are digitized along the roads while recording the GPS positions. Items like manhole covers and fire hydrants are mapped as points with associated attribute information. Inspection and maintenance crews use GPS to navigate directly to sites needing attention. Road conditions, hazards, and areas that need repairs are entered as attributes for use as inventory and GIS programs. The potential advantages of using GPS for transportation surveys are numerous (Adamu, 2003). They include:

- Automatic collection of trip origin, destination and route data

- Routes recorded for all trips, allowing for the post processing recovery of unreported trips 
- Accurate trip start and end times, as well as trip lengths automatically determined

- Verification of self-reported data

- Passive traces of vehicle routes over multiple-day periods

- Enhanced understanding of observed travel patterns and the decision process related to travel

- On-time information for policy evaluation and cost benefit analysis

GPS can be used for any application that requires accurate time, position and other feature information. The final output is not limited to plots and maps. Position and time records can also be transferred to software programs that require the information for modeling functions. One very useful application for the GPS is to maintain a common reference system for data collection. A frequent concern when using GIS databases is the need for a common reference system between different data sets. Data collected with GPS can be accurately referenced to control points in a geodetic survey network. GPS uses WGS-84 (World Geodetic System - 1984) as the common reference system.

The next level of technology will be to overlay these positioning and guidance systems with current information on traffic conditions including crashes and incidents, to provide real time assessments of the best routes available between two points. The progress in technology for data collection and analysis is rapid and thus transportation engineers have the responsibility to keep up with the state-of-the-art in traffic data collection, reduction and analysis techniques so that they may apply the best possible tools to obtain needed information concerning transportation planning and design. Some of these techniques will be applicable to the non-motorized modes of transportation, as discussed below. 


\subsection{Pedestrian / Bicycle Travel Data}

Pedestrian and bicycle travel has become increasingly important in the United States in recent years as traffic planners recognize its potential to provide mobility, reduce congestion, improve environmental quality and also to promote public health. The Transportation Equity Act for the $21^{\text {st }}$ Century (TEA 21), passed by Congress in 1998, required the consideration of pedestrian and bicycle needs in transportation planning and also directed the Bureau of Transportation Statistics (BTS) to develop data on bicycles and pedestrians. $\quad$ Research, planning and policy-making efforts to improve conditions for pedestrian and bicycle travel require a variety of data such as travel and facility characteristics (location and attributes), facility usage (volumes, route, purpose), crash and safety information and user preferences. However, deficiencies and limitations in existing sources of data and data collection techniques have been a barrier to the appropriate level of development and/or improvement in these non-motorized modes of travel. This section of the literature review summarizes these data issues.

The report Bicycle and Pedestrian Data-Sources, Needs and Gaps (USDOT, 2000), is one of the most comprehensive compilations on collecting pedestrian and bicycle data. The document outlines the many uses of pedestrian and bicycle data. A perspective on the various uses of bicycle and pedestrian data is helpful in assessing needs and opportunities. The data collected for these non-motorized means of travel can be applied to three broad uses:

- Research studies and recommended practices

- Planning and design of facilities, project selection decisions, policies and programs.

- Analysis of conditions and trends to inform policymaking. 
The types of data collected and the various uses of those data can help shape data collection programs to benefit the greatest number of users. Data sources need not exist only at the national level, but also at the state and local levels, varying with the type of data required. The USDOT report (2000) identifies the following categories of pedestrian and bicycle data:

- Usage, trip and user characteristics, including counts of users, trip patterns, and demographic socioeconomic characteristics.

- Data on the preferences, needs, and attitudes of current or potential bicyclists and pedestrians.

- Facility characteristics, including the locations and attributes of pedestrian and bicycle facilities

- Crash and safety data, including crashes and other data related to the safety and security of pedestrian and bicycle travel.

- Expenditures on and capital stocks of facilities.

This literature review concentrates primarily on usage, trip and user characteristics since the other categories fall outside the scope of this study.

\subsubsection{Data on Usage, Trip and User Characteristics}

This category of data answers the questions of who is traveling, how, where, when and why. The data may take the form of number of travelers by facility, time of day or geographic area. Data on trips include items such as origin and destination, trip length, mode, route, purpose and time of day. Characteristics of the trip maker or 'system user' may include demographic and socioeconomic factors such as age, gender, 
income, household structure, and other information that describes the person and his or her situation in life.

Data may be reported either in aggregate or disaggregate form. Disaggregated data include records of the characteristics and trip patterns of individual people or households. Disaggregated data can be analyzed directly for modeling purposes. Data on usage, trip, and user characteristics are initially collected at a disaggregated level but are frequently reported or available for analysis only at some level of aggregation. In the aggregate form, personal and trip variables may be cross classified, e.g., trip length distributions by mode, purpose, and/or age. Data sources for usage, trip and user characteristics include:

- Counts of bicyclists or pedestrians - counts indicate the number of pedestrians or bicycles that may be using a particular facility at any given location, and can be differentiated by user behavior, usage by time, geographic area, facility type or user type. Unlike, the Highway Performance Monitoring System (HPMS), which is a national system for tracking motor vehicle traffic volumes, no such equivalent exists for monitoring pedestrian and bicycle volumes or the distance traveled. While a few cities and MPOs routinely conduct pedestrian and bicycle counts, most collect them only sporadically for specific studies (e.g. off-road trail usage study) or do not collect them at all.

- The U.S. decennial census - The decennial census represents a nearly 100 percent enumeration of the U.S. population, although some variables are collected only for a sample of the population. Data of interest to bicycle and pedestrian planning include work trip characteristics- notably mode, travel time, time leaving for work, time 
leaving from work, and origin /destination areas. Census data are widely used in transportation planning as they are publicly available, are easy to use and are reliable due to the large sample size.

The USDOT report (2000) presents some of the limitations of census data for pedestrian and bicycle analysis:

- Only work trips are included. Thus, if census data are used to represent overall levels of walking and bicycling, these must be assumed to be in some proportion to work-trip use. Work trips make up less than one quarter of all trips; so non-work trip-making patterns are not captured by the census.

- The data may not represent "normal" pedestrian and bicycle work-trip mode use.

- Some of the surveys are carried out infrequently and thus, they are not suitable for tracking short-term trends and changes.

- The Nationwide Personal Travel Surveys (NPTS) - NPTS is a household travel survey administered by the USDOT and the FHWA on the national level, every five to seven years. The NPTS is used to analyze the nature and amount of personal travel, the relationship between socioeconomic characteristics and travel patterns, and trends in passenger travel. The NPTS is large enough to provide meaningful information for pedestrians and bicycle trips at the aggregate level by trip purpose and trip rates by mode, and social, economic and demographic characteristics of the respondents. The NPTS is not detailed enough to identify bicycle and pedestrian trip and traveler characteristics for specific geographic areas, such as a metropolitan area. Increasing the sample size to allow for more geographic specificity presents 
significant cost implications, just as it does with metropolitan household surveys. Modifications similar to those noted above could be made for metropolitan household travel surveys, including over-sampling areas with high levels of pedestrian and bicycle travel, and improving the questionnaire and providing better interviewer training to reduce underreporting of short-walk trips and transit-access trips. However, longer surveys can suffer from a reduction in quality and a lower rate of response, all of which must be considered along with cost implications when designing the survey.

- Metropolitan household travel surveys - Such surveys are conducted in larger metropolitan areas by the MPOs and follow the method of filling out travel diary surveys. The surveys are administered to a sample of 1000-or more households whose members record their trip patterns (e.g., time, origin, destination, mode and purpose) over a course of one or two days. Data collected are used in transportation planning to predict future travel patterns, assess transportation needs, and examine the effects of various policy decisions.

- Other surveys conducted sporadically at a national level - Characteristics of pedestrian and bicycle travelers can also be obtained from various other national-level surveys that are conducted occasionally. Some of the surveys are Consumer Product Safety Commission's (CPSC) national adult bicyclist survey, recreational surveys and NHTSA's survey on public beliefs.

- Various local surveys and market studies - Local surveys, in some areas, are conducted of bicyclists and/or pedestrians to assess personal and trip characteristics. University researchers, city or metropolitan planners, or other interested groups may 
conduct such surveys. The content, scope, and quality of these surveys vary considerably according to the specific purpose, budget, and level of knowledge of those responsible for the survey. Nevertheless, these surveys can yield useful information for local planning.

The counts described above are obtained using various types of modern pedestrian and bicycle traffic counters, which record the volume of traffic at a point on a facility. Most of the surveys described are conducted using the travel diary questionnaire forms that are distributed to subjects. Both of these data collection methods are described in the next section.

\subsection{Conventional Methods of Data Collection}

\subsubsection{Counting Devices}

Most traffic detection and counting technology and sensor research have focused on the detection of motorized traffic. This has left a need for objective information on the performance of detectors and counters for non-motorized traffic. Bicycles and pedestrians are the two most common types of non-motorized traffic. Recently, sensors have been developed to detect the presence of pedestrians and the speed and presence of bicycles. The U.S. Department of Transportation (2000) recently published Bicycle and Pedestrian Data: Sources, Needs and Gaps. This in-depth study identified volume as one of the most critical transportation data requirements. Volume data or count data can provide specific information on time of day, day of week, geographic area, or facility type and usage. The study identified the following use for historical volume data:

- Tracking trends in usage

- Evaluating level of service on a facility by comparing volumes to capacity 
- Identifying and prioritizing improvements

- Calibrating travel demand models

The volume of non-motorized traffic can be determined with a variety of counters using different technologies, including microwave, infrared, video and inductive loops. These will be discussed in the following sections.

Various types of devices are available to count non-motorized traffic (Gasvoda, 2002). Gasvoda states that most of these counters are widely used on trails to detect and record the presence of pedestrians and bicycles. These counters not only detect the presence of a pedestrian or bicyclist but also keep count of the traffic with the help of a data logger that records data simultaneously. There are a few general problems identified and associated with the counters. These problems may be more evident for some counters and less for others. Gasvoda (2002) has identified the following issues of concern relative to counting devices:

- Accuracy

- Installation and sensitivity adjustment

- Maintenance and battery life

- Vandalism

- Weatherization and equipment malfunction

- Costs

- Unable to distinguish between human and animals, and between individuals and groups

- Difficulty in post processing of data

- Directional capability 
- Effectiveness with the time of day

- Time of count

The primary types of counters using different detection techniques are:

- Infrared - Infrared counters may be further divided into:

1. Active Infrared systems where the scanner emits an invisible infrared beam of light across the trail or path to a reflector. The reflector returns the light beam back to the receiver. If the beam is broken, presumably by a trail user, a count is registered. The size of the beam that must be interrupted to register a count is roughly the size of the reflector (about three inches). To eliminate false counts from falling leaves, snowflakes or birds, the beam must be blocked for a minimum length of time before a count is registered. The active infrared sensor is mounted at height of about 3-4 feet and thus can be used to monitor pedestrians, equestrians, off-highway vehicles, bicyclists, skiers and snowmobilers. Since the mounting height is $3-4$ feet, false counts due to small animals such as dogs and cats may be avoided. On the other hand, the mounting height may also preclude counting children, which would give an inaccurate count.

2. Passive Infrared systems operate by detecting a moving object's infrared signature. Factors that determine the signature include the object's temperature relative to the background, its infrared reflective and emissive characteristics, and its size, speed of travel and direction of travel relative to the counter. The infrared energy can originate from the object or can be reflected by the object. Because of the complex interaction of these 
factors, the detection range varies considerably. Large, fast-moving objects at a temperature much different from the background are detected at the longest range. These have multiple detection zones through which an object must pass to be counted. The angular beam width and number of zones are determined by the manufacturer, and the size of the zones increases as the distance from the counter increases. At a reasonable installation distance, passive infrared counters cannot discriminate people in a group and will undercount. They are also prone to false counting because of changes in the background. They are normally mounted at a height of 3 feet.

- Seismic - These systems consist of a counter unit and a buried vibration sensor or geophone. Often a mat or tube helps carry the vibrations to the sensors. As trail users walk down the trail, their footfalls cause vibrations detected by the sensor and a count is registered. The buried seismic system is undetectable and may be well-suited for areas where vandalism is a problem. Spike sensors are geophones with a spike on one end that is poked into the ground. The sensors can detect hikers up to 30 feet from the sensor in compacted soil. The spike sensor is the least accurate seismic unit and should only be used where knowledge of general numbers or trends is desired.

- Inductive loop - These counters detect metal objects such as bicycles and wheel chairs, which, when passing over loops buried under the path, induce a brief current in the loop which is recorded as a count. The inductive loop counters 
cannot be used for detecting pedestrian traffic, as they require metallic objects to induce current.

- Pressure pads - This technique uses a rubber mat pressure pad that is buried up to $800 \mathrm{~mm}$ below the path surface, and registers a count when trodden on. Different versions can either record all users or distinguish between walkers and cyclists. The devices are usually data loggers, which may be directly connected to a computer. Rubber pads are susceptible to damage from sharp stones, and do not work if ground is frozen or covered with snow.

- Radio Beam counters - This counter uses a radio beam that is generated and detected by two units placed at either side of the path. The radio beam needs something solid (i.e., a person) to break it, so they are less susceptible than infrared beam counters to false counts from leaves and snow. They record data on logger units. These must be attached to a computer for reading. They can be set to count any two out of the three users: pedestrians, bicycles and horses, by measuring the time the beam is broken. The units are compact and use little battery power.

- Thermal Imaging - These detectors are relatively new types of counters that use thermal imaging to identify the size, location, speed, number and direction of people. These devices detect the heat given off by moving people and objects, and should work equally well indoor and outdoors in all weather and also in complete darkness. These sensors are placed over the path, and detect people passing underneath. 
In recent years, sensors have also been developed to detect the presence of pedestrians at intersections and the speed and presence of bicycles. This has been accomplished with a variety of technologies, including microwave, ultrasonic, infrared, video and inductive loops. With the development of ITS applications, automated pedestrian detectors are beginning to complement the existing push button detectors. These applications optimize intersection operations and improve safety by reducing the conflicts between vehicles and pedestrians.

The report by SRF Consulting Group, Inc. (2003) prepared for the USDOT and the Minnesota DOT documents the state of pedestrian and bicycle detection and presents evaluation results from a field test conducted of six different bicycle/pedestrian sensors. These detection devices are sensors which detect presence of pedestrians or bicycles and may be used as counting devices when attached to data loggers that keep the count. The primary goal of this project was to identify the applications and evaluate the accuracy of different non-intrusive technologies in detection of non-motorized traffic. It identified the applications for non-motorized traffic and listed similar projects that had been conducted for this purpose. It also evaluated the participating sensor performance.

\subsubsection{Travel Diaries}

Axhausen (1995) defines a travel diary as "a survey instrument designed to record all movements of a person over a given period of time with all relevant detail for the relevant time horizon, considering travel and non-travel as two distinct classes of activities". Data collected from thousands of households across a region are analyzed to estimate current travel demand and to predict future travel demand. The accuracy and completeness of this travel data obviously have a critical impact on model results. 
These travel diaries are typically used for planning purposes. Travel diary entries can be either in the form of information about the trip such as origin, destination, time of day and trip duration, or of responses to prepared questions relevant to information required.

As mentioned earlier, TRB (2004) states that travel surveys often involve a twostage process: a recruitment interview to obtain information about the household, followed by an extended data gathering interview to collect information on household travel. The complexity and content of household travel surveys impose a significant burden on respondents and thus can result in a substantial fraction of non-respondents. For example, to understand travel patterns and to provide data for estimating travel behavior models, travel surveys collect household and individual demographic characteristics as well as detailed descriptions of all trips taken during a specific time period.

Trip data that are needed for most or all persons in the household include origin and destination, purpose, time of day, mode of travel and other characteristics. A household may make as many as 20 or 30 trips in a day. Recalling and reporting on these trips involve major effort on the part of the respondents, and there is some evidence that overall response rates decrease as a result of greater respondent burden. Also, the changing characteristics of telephone usage are reducing the effectiveness of current telephone survey methods and may be introducing bias. The travel survey forms are accepted as data collection techniques for data collection at the level of the census tract, city and metropolitan area. 
Unfortunately, not much literature was found on the use of travel diary surveys for collection of information on pedestrian and bicycle movement. Note that information gathered from household surveys such as Metropolitan Household Travel Surveys is, in theory, useful for assessing characteristics of pedestrian and bicycle travel. In practice, these surveys also suffer from a number of limitations:

- Trips solely for the purpose of recreation, such as a stroll or bicycle ride, are not included.

- Walk trips (particularly short trips such as from work to a cash machine) are commonly underreported. Also, walk trips to access other modes, notably transit, are often not tracked separately in travel surveys.

- The surveys provide only limited information on the travel patterns of children. The parent completing the survey is generally asked to report trips for children from age five to somewhere in the teens. As a result of this proxy reporting, not all trips may be noted.

- Lastly, many MPOs cannot afford to conduct expensive travel surveys. For those that do conduct regular surveys, the sample size and trip reporting are often too small to provide useful information for detailed bicycle and pedestrian planning. In addition to the limitations mentioned above, the second stage in travel surveys, i.e., extended data gathering interviews to collect information, also faces a few problems (Transportation Research Board, 2004):

- Consumer resistance - unsolicited phone calls, especially telemarketing calls, compete for respondents' attention and have a negative effect on response rates. 
- Cell-phone-only households - cell phones are excluded from random digit dialing which may lead to a sampling bias because of the increasing number of cellphone-only households.

- Language difficulties - the number of households in the US for which the first language is not English is growing, thus making telephone communication far more difficult for survey respondents who are not native English speakers.

Non-response is a major concern because of the potential for bias and the resulting implications for data quality. TRB (2004) suggests that, to overcome nonresponse and other data quality challenges, there is a need for multiple data collection methods that use different ways to reach people and measure their behaviors.

As such, more sophisticated travel survey methods along with search for both better data and less expensive data collection data methods are being developed. It can also be gathered from the report (TRB, 2004) that full automation of travel survey data collection processes should produce more data and more accurate data. Some encouraging results have been obtained using internet-based travel diary surveys and handheld electronic travel diaries (TRB, 2004). Internet-based travel diaries permit the implementation of user-friendly features such as context-sensitive instructions, a help feature, automatic addition of intrahousehold shared trips, and the ability to complete the diary in a series of work sessions at times convenient to the respondent. But, such internet-based diaries may also suffer from a few drawbacks. The reason for developing these diaries on the internet was to make the task of keeping logs easier and to obtain accurate data. Since these internet-based forms again are filled by the subjects, they are prone to being inaccurate. For example, the location of the subject is not known 
automatically but has to be fed in by the respondent himself, thus creating potential for inaccuracy. TRB (2004) also mentions that the up-front design cost of such internetbased travel diaries is relatively high, and such diaries are susceptible to disruption by computer viruses and hackers. The report (TRB, 2004) also says that such diaries are limited to households with internet access and have an associated socioeconomic bias, with web respondents having higher incomes and being younger than population average.

Currently, global positioning system (GPS) technologies (in-vehicle) have introduced a new level of comprehensiveness and accuracy to traditional travel diaries (Wolf, et al., 2001). Various types of data (such as position, speed, heading and altitude) can be recorded automatically without burdening the respondent for the data and thus can be used to verify the self-reported data. In Wolf, et al. (2001), the procedure for using the in-vehicle GPS equipment package involved its installation in the vehicle. Once installed, the GPS receiver attempted to acquire satellite signals as soon as the vehicle was started and automatically stopped receiving data as soon as the vehicle was turned off. Data logger, the GPS data-logging application, was installed on each palm device. The respondents were instructed to power on the palm device each time they started their vehicles. Data logger was programmed to record second-by-second GPS data while movement was detected.

Although several studies have been conducted to evaluate the use of the GPS with either paper travel diaries or electronic travel diaries, these studies considered GPS data only as a supplement to the traditional travel diary elements collected in the diary itself (Wolf, et al; 2001). The use of GPS technology as a means to collect non-motorized travel data will be discussed in detail in the latter part of the review. 
A number of data collection techniques and devices are used these days, but most of them are used to obtain counts for usage of individual facility. Therefore, more methods and devices should be analyzed which give more information about personal travel and individual usage characteristics. A few devices that are going to be used for the study are discussed further on in the review.

\subsection{Wearable Devices}

\subsubsection{Pedometers}

Pedometers are small, motion sensor devices that count the number of steps taken during locomotion (Freedson and Miller, 2000). Vertical accelerations that exceed a specific threshold cause a lever within the device to be triggered registering a step or count (Welk, et al., 2000). Pedometers are clipped to a belt or waist band above the midline of the thigh. According to the Consumers Union (2004), they must remain snug, so they do not bounce around, and vertical, for accurate readings. Early pedometers were mechanical and subject to relatively large errors, making them unsuitable as precise research instruments. Most pedometers today are electronic, and are activated when the foot hits the ground, producing an impulse that transfers to the pedometer case. The pedometer contains a lever arm with a pendulum attached to a spring. With each step, the pendulum moves and one electrical event is recorded on the digital readout. The output given by the pedometer is usually a count of steps, but newer models are able to estimate distance traveled and caloric expenditure, given appropriate information about stride length and body weight, respectively. Although pedometers are unable to measure temporal patterns of walking or any other physical activity, a few of the newer pedometers include a stopwatch and clock. 
To determine distances traveled from the counts given, additional information is required from subjects such as walking stride length and weight (for physical activity purpose i.e. to calculate calories burned). For some pedometer models, this information can be directly fed into the device (Tudor-Locke and Myers, 2001). The calibration of the stride length is done by asking a subject to walk ten paces (in a normal walking behavior) in a large hall or room, and this distance from start to the end is measured with a tape. This distance is then divided by ten to get the normal stride length, which is fed into the pedometer and used to calculate distance.

There are a number of advantages associated with pedometers that make them worth considering as a data collection tool for transportation planning. Besides being relatively small and lightweight, they are also inexpensive, with cost ranging from approximately $\$ 15$ for simple ones that simply count the number of steps to $\$ 45$ for more sophisticated models that not only count steps but, have other features such as distance walked, calories burned, stop watch and clock. Pedometers are not intrusive and virtually all segments of the population can wear them. Pedometers are also socially acceptable (Sequeira, et al., 1995) and relatively simple to operate. People from diverse cultures and languages can use pedometers because they provide objective numerical measurements (Sequeira, et al., 1995). Unlike subjective measurement tools such as questionnaires, diaries, and logs, pedometers avoid self-report bias. Pedometers can thus provide an objective measure to help validate self-reported estimates of walking (Dishman, et al., 2001).

Numerous studies have been conducted to evaluate pedometers as a means of promoting physical activity and motivating patients to walk for good health. Tudor-Locke 
(2002), citing other work, indicated that pedometers have been used in Japan to assess physical activity and increase walking behaviors for over 30 years. At least 10 Japaneselanguage articles on pedometers are currently listed in PubMed (an electronic search engine). Using other search strategies (communicating with Japanese collaborators, translating reference sections of held Japanese language articles, translating references obtained through Japanese-language search engines) yielded another 48 promising articles. Tudor-Locke (2002) also observed that currently, Japanese-language literature about pedometer-assessed physical activity represents an untapped source of scientific and practical information. Unfortunately, without translation, the contents of these articles are inaccessible to most North American researchers and practitioners. Support for the translation and review of previously inaccessible Japanese-language literature will likely contribute much to our understanding and use of the pedometer for multiple practical purposes.

Studies involving use of the pedometer as an instrument to collect pedestrian travel data were rare in the published literature. Among the very few Japanese articles translated to English, one by Koike, et al. (2001) used pedometers to study pedestrian travel patterns in different cities and also analyzed patterns of walking in different city districts. Pedometers were used to measure steps of pedestrians, with varying builds and age groups, in different cities and, using different facilities to commute to different districts in a city. Although the translation did not clearly convey the details of the study, it appears that pedometers were used to determine the amounts of walking associated with different modes of transportation. The authors concluded that the average number of steps for the movement between facilities indicated the level of public transport in a 
city. According to Koike, et al. (2001), the results showed that the number of steps at the time of movement between facilities changes with the mode of transport. For example, the walking mode would involve the greatest number of steps with motor vehicle travel involving the fewest steps. Since public transport would involve some walking to/from the station or stop, a step count difference between those found for walking and driving would be expected. The same study also compared the public transport infrastructure of two different cities, and concluded that the distance traveled on foot also varies with how developed the public transport infrastructure is in each city.

Despite the obvious benefits of pedometers, they also have a several limitations with respect to travel data collection. The pedometer counts the number of steps and, thus, calculates the distance traveled by the individual once the stride length has been incorporated. This stride length is measured and determined beforehand, which may be approximate and inaccurate. The pedometer registers a step when there is a movement in the leg, which acts as a disadvantage since any motion made by the leg, even while sitting, can be recorded as a step. In addition, the pedometer is unable to differentiate between walking step and steps taken while running or using stairs. The pedometer assumes that all stride lengths are equal, so the distance may be underestimated if the individual chooses to run instead of walk since fewer steps will be used (and recorded) to travel the same distance.

Thus, one of the issues associated with pedometers is their accuracy, i.e., how well the device's step count compares with actual step count. The Consumers Union (2004) tested twelve pedometers and three speed-and-distance monitors on a treadmill. They found that only three pedometers were accurate within 5 percent at 2.5 miles per 
hour and only one speed-and-distance monitor provided the distance within 5 percent. "Most of the other devices were off by 5 to 10 percent and a few pedometers were off by 20 percent or more". The pedometers did much better at 3.5 miles per hour. All but one pedometer were accurate to within 5 percent. The study found that while pedometers are easy to clip on, accuracy can be adversely affected if the device is not worn properly.

The work of Bassett, et al. (1996) also showed that pedometer accuracy was adversely affected by slow walking speeds. This part of the study was carried out on a treadmill. Results showed that at slower speeds, there was error in the values obtained on the pedometer in comparison to actual readings. Another part of the study determined accuracy by comparing pedometer readings for similar brands worn together but on the left and right side of the body. The report concluded that one brand of pedometer was significantly more accurate than others for studies involving step counts. This report has also become the reference for various other articles using the same brand of pedometer in their studies. For example Welk, et al. (2000) used the pedometer brand said to be most accurate in his study assessing physical activity.

Literature reviewed for this study (Bassett, et al., 1996 and Consumers Union, 2004) showed that the accuracy of the pedometers was determined using treadmills at 0 percent grade for different speeds. The Consumers Union (2004) results were based on data from eight volunteers. The accuracy obtained for different pedometers on a treadmill can be different from situations in the actual walking environment where there are gradients, irregular surfaces and other pedestrians. The stride length may change on gradients (varying with the percent grade) or with the type of surface. This may be of significance as the number of steps needed to traverse a given distance changes. 
Pedometers cumulatively record steps. Almost none of the current models are able to store the number of steps in a particular trip or a specific day over several days. Thus, if a single trip or daily records are important (as they would be in transportation planning applications), the pedometer must be reset manually. Most pedometers do not contain an internal clock nor are they able to store data (other than steps) internally (Bassett, et. al; 2000). Therefore, step counts from the device need to be recorded in a $\log$ or diary on a regular basis if data are to be collected over an extended period of time, or if trip or daily information is required. Similarly, current pedometers do not have the ability to store origin and destination information. Such information would also have to be a part of a supplemental trip log. Difficulties associated with participants maintaining travel diaries or logs have been discussed previously. In this case, the difficulty is related more to getting the participants to make real-time entries in the log so that recollection of walking trips from memory is not needed.

A recent review of newer technology relating to pedometers showed that more sophisticated and technological advanced pedometers are now available in the market. These advanced pedometers now contain data storage capabilities in which the steps taken during a given activity period varying from three days to up to seven days can be counted and stored. They also include a built-in clock. One example of such a pedometer is the Omron-HJ70ITC. Another device that was reviewed was the GPS pedometer. An example of such a device is the Navman Sport Tool W300. This device uses the accuracy of GPS technology to give information on speed and distance. It gives precise measurement on the distance covered, current, maximum and average speed and 
calories burned. It also has a timer to measure the time walked, and also provides precise date and time stamp.

A number of articles have been published on the use of pedometers for increasing physical activity, but very few articles talk about their accuracy and reliability relative to collecting travel data. Bassett, et al. (1996) used a three-part study to compare the accuracy of five different brands of electronic pedometers. Accuracy referred to measuring the distance walked and number of steps correctly. In one part, the effect of walking speed on the step count shown by the pedometers was examined. The results of the study showed that at very slow speeds the pedometers tended to underestimate the distance. This was due to a 'failure to register' foot strikes. The Yamax pedometer was more accurate at slower speeds. Similarly, at faster speeds also, the pedometers underestimated the distance. This error was due to a lengthening of the actual stride length rather than a miscounting of steps. Thus, it is fair to conclude that pedometers are sensitive to the rate of walking.

In another part of the study conducted by Bassett, et al. (1996), accuracy was determined on two different surfaces, i.e. rubberized surface and concrete surface. The results showed that there was no significant difference in distance recorded for different walking surfaces. It can be hypothesized that different surfaces, such as grass, unpaved surfaces, deteriorated sidewalks and snow-covered surfaces, may also affect stride length and, therefore, pedometer accuracy. No published literature could be found that determined the effect of gradients, which may also affect stride length. Other factors that appear to affect stride length such as social interaction among pedestrians and the effect of other pedestrian traffic have not been considered in studies of pedometer accuracy. It 
would be appropriate to ascertain the effects of these factors on pedometer accuracy, as part of any assessment to determine the feasibility of pedometers as a tool for travel data collection.

\subsubsection{GPS Devices}

This section discusses the technology associated with two of the devices used in the study. The section starts with a description of the technique and how it is being used currently. Next, studies conducted using GPS data-loggers (in-vehicle) for collection of travel data are reviewed. The last topic is a brief description of the devices used for the study and how they are being used to collect data for transportation planning.

Global Positioning System (GPS) is a satellite-based radio positioning system that uses the United States Department of Defense satellite system to accurately provide position, velocity and time information to those with access to a GPS-enabled device. These GPS satellites are also known as NAVSTAR (acronym for NAVigation Satellite Timing and Ranging). These 'users' can acquire data about their location, speed or time. Trimble Navigation Limited (2000) describes the GPS as a worldwide radio-navigation system formed from a constellation of 24 satellites and their ground stations. GPS uses these "man-made stars" as reference points to calculate positions accurate to a few meters. When fully deployed, GPS provides all weather, worldwide, 24-hour position and time information to the user, depending on the purpose for which the GPS is being used.

This category of technology includes a variety of GPS-enabled devices. One of the most popular civilian uses of GPS is for hiking, trekking and fishing. The GPS Primer (Aerospace Corporation, 2003) describes some of the GPS uses in everyday life. 
Sophisticated GPS units are now available equipped with detailed maps of city streets and building information. Automobile manufacturers are offering moving-map displays guided by GPS receivers as an option on new vehicles. These units have made wayfinding and navigation easier. The Primer (Aerospace Corporation, 2003) also describes how vehicle tracking is becoming one of the fastest-growing GPS applications. GPSequipped fleet vehicles, public transportation systems, delivery trucks, and courier services use receivers to monitor their locations at all times. The use of GPS to track workers and vehicles is catching on with a growing number of business and government employers, bent on improving productivity and customer service and on keeping tabs on labor costs. Many police, fire, and emergency medical service units are using GPS receivers to determine the police car, fire truck, or ambulance closest to an emergency, enabling the quickest possible response in life-or-death situations. In the field of wildlife management, threatened species are being fitted with GPS receivers and tiny transmitters to help determine population distribution patterns and possible sources of disease. GPSequipped balloons are monitoring holes in the ozone layer over the polar regions, and air quality is being monitored using GPS receivers. Buoys tracking major oil spills transmit data using GPS. Landry and Oliver (2002) discuss another purpose for which GPS technology is currently being used, i.e., to develop stormwater infrastructure inventory of stormwater assets such as pipes, inlets and manholes.

A currently popular application for GPS users is the adventure game Geocaching. The basic idea is to have individuals and organizations set up caches or small treasures all over the world and share the locations of these caches on the internet. 
GPS users can then use the location coordinates to find the caches. The website Geocaching.com gives detailed information on this popular adventure sport.

Among transportation engineering applications, GPS along with Geographic Information Systems (GIS) is being used for collecting travel time data. GPS is used for corridor identification and traffic monitoring, while GIS is used for data storage, retrieval, and visualization (Bullock, et. al, 1998). GPS technology has also been used to collect travel data for both motorized (Stopher, et. al, 2002a, Stopher et. al, 2002b, Wolf, et. al, 2001) and non-motorized means (Stopher, et al, 2002a, De Jong and Mensonides, 2003).

Several recent pilot studies have combined GPS technology with travel survey data collection to evaluate opportunities for improving the quantity and accuracy of travel data. These studies have used GPS (in-vehicle) devices to supplement traditional data elements collected in paper or electronic travel diaries. Although many traditional trip elements such as speed, time, distance, altitude and position can be obtained from the GPS data, trip purpose has remained an elusive element, requiring the use of a diary to collect. Wolf, et al. (2001), describes one study conducted by the Georgia Institute of Technology that examined the feasibility of using GPS data loggers (in-vehicle) to completely replace, rather than supplement, traditional travel diaries. In this approach, all GPS data collected by the data-logging device was processed so that all essential trip data elements such as position, speed, time, distance, including trip purpose were derived. The study used GPS data loggers to collect travel data in personal vehicles. The GPS data were then processed within a geographic information system (GIS) to derive most of 
the traditional travel diary elements. The list shown below contains the preliminary matching of the key travel diary elements to the GPS elements:

- Trip origin address : GPS latitude and longitude

- Trip start time : GPS first second of movement

- Trip destination address : GPS latitude and longitude

- Trip finish time : GPS last second of movement

- Travel distance : GPS points and GIS links

- Trip purpose : GIS origin and destination land uses

These derived data were compared with data recorded on paper diaries by the survey participants and were found to match or exceed the reporting quality of the participants. Most importantly, this study demonstrated that it is feasible to derive trip purpose from the GPS data by utilizing a spatially accurate and comprehensive GIS.

One of the studies conducted by Stopher, et al., (2002a) tries to develop a passive GPS device, for which additional non-GPS data may be added through inference or through a subsequent prompted recall survey. The study uses both in-vehicle and wearable GPS data-loggers. Experiments were performed to test the devices for a range of different modes such as collecting data on trains, buses, personal vehicles and ferries. In addition, data were analyzed to investigate congestion and the amount of time spent under congested travel conditions. A prompted recall survey for additional desired data such as trip purpose, occupancy and trip costs, for each trip identified from GPS data was conducted between two to twenty days later. This procedure had the disadvantage that it relied on the memory of the respondent and the ability to recall all information desired. The study described the in-vehicle GPS device in several modes of transportation but did 
not provide any guidelines on how the wearable device should be used. Only one subject used a non-motorized mode of travel.

The other paper published by Stopher, et al., (2002b) addressed the issue of how to present the information contained in the records of the GPS data-logger device so that it is understandable both to survey respondents and to decision-makers. The paper also dealt with the issue of how to present to analysts and decision-makers, additional information that is present in the records, but that may not be needed in interacting with respondents. The paper described the use of GPS device, the paradigms used to convert the track points to coherent trips, examination and correction of the visualized travel, and presentation of the resulting maps and other visual tools to subjects in a prompted recall survey.

Another study using a GPS device (in-vehicle) for collecting a variety of travel data and trip information was conducted by the California Department of Transportation. Trip information is used by the state and various local and regional agencies for a number of planning purposes, including development of travel models (Adamu, 2003). In the late 1990s, pilot studies had confirmed that GPS technology could improve the accuracy and completeness of travel data. Caltrans was one of the pioneering agencies that initiated the GPS component of the California Statewide Household Travel Surveys - the first large-scale GPS-enhanced travel survey of its kind. The plan allowed for in-depth analysis of weekday travel, while controlling for household, person and travel characteristics in three regions.

De Jong and Mensonides (2003) used the wearable GPS data-logger as a datacollection method. This study builds on earlier work by Stopher and others on the use of 
passive GPS devices, for which additional non-GPS data may be added through a subsequent prompted recall survey. The paper presents sets of rules which can be applied to the raw data acquired by wearable GPS devices to determine the modes of travel used and trip ends. The study presented experiments in which the GPS device was tested for a range of different situations, including collecting data on trains, buses and ferries. De Jong and Mensonides (2003) also describe experiments for collecting data in urban canyons and also with respect to the cold start phenomenon. The paper also described the procedures undertaken to download and analyze the data.

The paper talks about the 'urban canyon effect'. Bad results in the data collected by the GPS data-logger were observed because of urban canyons or high buildings in the city center. The satellite transmissions were bounced around by these buildings (like sound echoes in a canyon). When a wearable GPS device picked up a bounced transmission, it calculated a wrong position because the transmission was also in the wrong position'. Because of this, the positions shown by the wearable unit did not reflect the actual location. This can also happen in other areas with closely spaced tall buildings.

This study also introduced the concept of 'cold start'. As described by De Jong and Mensonides (2003), "the GPS unit stores data about where the satellites are located at any given time. This data is called the almanac. Sometimes, when the GPS unit is not turned on for a length of time, the almanac can get outdated or 'cold'. When a wearable unit is turned on for the first use of a day or after it has been out of communication for 4 to 6 hours, a cold start will take place. It will then take the GPS receiver longer to connect with the satellites. De Jong and Mensonides (2003) found that such cold starts can take up to 28 minutes depending on the surroundings and the speed of travel. While staying at 
a fixed location, the cold start took less than 1 minute but when the receiver was moving at walking speed, it generally took 3 to 15 minutes to acquire a signal. During the cold start, no data will be recorded.

The study to be described in this thesis will evaluate the utility of "wearable" GPS devices for transportation data collection. One of them is a wearable GPS data-logger. A data logger is an electronic device that is connected to the GPS antenna/signal receiver and maintains a $\log$ of the data received by the antenna/receiver. It is a versatile device collecting detailed spatial and temporal travel data.

Another GPS device to be used in this study is a hand-held GPS, a device used primarily for outdoor recreation activities such as trekking, hunting, fishing and hiking. This device, when switched on, starts recording location data in its memory, as soon as it gets a location fix. A trail of these location data for every trip made is known as a track $\log$ for that trip. The hand-held GPS is capable of saving these track logs which can be used for navigation. Besides keeping a track $\log$, the unit's trip computer also provides the current speed, average speed, trip timer, trip distance and resetable maximum speed. A detailed description of the above two GPS-enabled devices, and their capabilities will be presented in Chapter 3.

A recent review of other GPS-enabled wearable devices showed devices which can be worn on the wrist and weigh no more than a watch. An example of such a device is the Garmin Foretrex 201. This device is recommended for outdoor activities such as hiking, sailing and paragliding. It incorporates almost all of the same features, if not more, as the hand-held GPS unit used for this study. 
In order to use the two devices, mentioned earlier, for collecting pedestrian data, it is necessary to address certain issues such as tracking capability, cost, user acceptability and post processing of data. The accuracy of GPS devices is directly related to the strength of the signal received from the satellites. The stronger the signal or the greater the number of satellites in contact with the device, the higher the accuracy. Thus, several preliminary tests will be carried out on these devices to verify if there is a loss of signal under various field conditions such as thick tree shade, between tall buildings, under high voltage power lines and in heavy rains.

The data collected by the GPS unit can be plotted on a map using a visual interface platform known as GIS. The Environmental Systems Research Institute (ESRI), the makers of Geographic Information Systems (GIS) package has defined GIS as 'an integrated collection of computer software and data used to view and manage information about geographic places, analyze spatial relationships, and model spatial processes. A GIS provides a framework for gathering and organizing spatial data and related information so that it can be displayed and analyzed'. Chang (2002) has described GIS as a computer system designed to capture, store, update, manipulate, analyze and display the geographic information. A GIS is typically used to represent maps as data layers that can be studied and used to perform analyses. Position, time and attribute information (for GIS databases) is collected by walking, riding, driving and flying around locations of interest. This study will concentrate primarily on the use of GPS technology with respect to data collection for transportation planning. 


\subsection{Concluding Remarks}

The literature review has shown that procedures and devices for collecting vehicular travel data are far more developed than those for collecting non-motorized travel data. This is logical, since up until recently, motor vehicles were the primary focus of transportation planners and engineers in the US. The potential of pedestrian and bicycle travel as legitimate transportation modes is being recognized, but travel data collection for these modes still lags that of motorized modes.

A number of methods for counting or determining the number of users on pedestrian facilities are available and new technological developments are occurring on a continuing basis. However, there is a need for non-motorized user-related information such as trip origins and destinations, travel-time, travel speeds and delays. While conventional transportation planning techniques such as travel diaries can be adapted to non-motorized modes, this approach generally suffers from a number of disadvantages and limitations. New technology, specifically GPS devices, appears to offer a viable approach to overcoming some of these problems.

Wearable devices have primarily been used for assessing physical activity of users and as a navigation aid by outdoor enthusiasts. However, only limited published information was found on these devices being used as a tool for pedestrian data collection. These devices, besides being relatively low-cost, are minimally intrusive yet can provide information such as speed, duration of trip and origin/destination of the user. Some of them also incorporate GPS technology. Thus, it seems logical to explore the use of wearable devices as methods to gather travel data for non-motorized modes. 
While wearable devices appear to have potential for this application, there are still a number of unanswered questions that must be addressed before their feasibility can be established. These include costs, data collection capabilities, accuracy, user acceptability and post-processing of data. In the absence of any published assessment of wearable devices, the proposed study will evaluate these devices under various field conditions to assess their utility in collecting data for transportation planning purposes. 


\section{Chapter 3}

\subsection{METHODOLOGY}

\subsection{Introduction}

The objective of this study was to assess the feasibility of three different devices: (1) pedometer, (2) hand-held GPS unit and (3) wearable GPS data-logger in collecting pedestrian data for transportation planning and engineering purposes. Before feasibility could be established, it was necessary to perform experiments to examine and evaluate these devices under field conditions. The experiments used in making the assessments are described here. This chapter of the report has been divided into two parts:

- Description of devices used

- $\quad$ Procedures and data collection for two categories of experiments:

- Device characteristics

- Operational considerations

The "description of devices used" section will discuss, in detail, the three devices, their specifications and capabilities and how they can be used for data collection. The experimental procedures section will discuss the procedures and criteria followed to conduct the various preliminary tests and experiments with the devices. The data collection section presents the data collection forms used in the experiments.

\subsection{Description of Devices Used}

This section describes, in detail, the three devices evaluated in the study. These devices were: a pedometer, a hand-held GPS unit and a wearable GPS data-logger. 
Through various experiments described in this chapter, the feasibility of using each of these devices in transportation planning and engineering was assessed.

\subsubsection{Pedometer}

As mentioned in the literature review, pedometers are wearable motion sensor devices that count the number of steps taken during locomotion (Freedson and Miller, 2000). The pedometer is a small, non-obtrusive device, usually attached to the individual at the waist or ankle. An individual can wear a pedometer by clipping it to a belt at the center of the leg. Vertical accelerations that exceed a specific threshold cause a lever within the device to be triggered, registering a step or count (Welk, et al., 2000). Most pedometers today are electronic and are activated when the foot hits the ground, producing an impulse that transfers to the pedometer case. The pedometer contains a lever arm with a pendulum attached to a spring. With each step, the pendulum moves and one electrical event is recorded on the digital readout. The output given by the pedometer is usually a count of steps, but newer models are able to estimate distance traveled and caloric expenditure. Although pedometers are unable to measure temporal patterns of walking or any other physical activity, a few of the newer pedometers include a stopwatch and clock. This study was concerned with the number of steps taken as shown by the pedometer during the experiment, rather than distance. If the number of steps is known, the distance can be calculated by multiplying the individual's stride length by the number of steps.

There are a number of reasons that make pedometers worth considering as a data collection tool for transportation planning. Besides being relatively small and lightweight, they are also inexpensive with cost ranging from approximately $\$ 15$, for 
basic units that simply count the number of steps, to $\$ 45$ for more sophisticated models that not only count steps but offer a variety of capabilities, including distance walked, calories burned, stop watch and clock. Pedometers are not intrusive and virtually all segments of the population can wear them. Pedometers are also socially acceptable (Sequeira, et al., 1995) and relatively simple to operate. People from diverse cultures and languages can use pedometers because they provide objective numerical measurements (Sequeira, et al., 1995). Unlike subjective measurement tools such as questionnaires, diaries, and logs, pedometers avoid self-report bias. Pedometers can thus provide an objective measure to help validate self-reported estimates of walking (Dishman, et al., 2001).

Pedometers cumulatively record steps. Current models are not able to store the number of steps in a particular trip or a specific day over several days. Thus, if a trip or daily records are important, the pedometer must be reset manually and a separate record kept of step count and/or distance. Most pedometers do not contain an internal clock nor are they able to store data (other than steps) internally (Bassett, et al., 2000). Therefore, step counts from the device need to be recorded into a log or diary on a regular basis if data are to be collected over an extended period of time, or if trip or daily information is required. Similarly, current pedometers do not have the ability to store origin and destination information. Such information would also have to be a part of a supplemental trip log. Difficulties associated with participants maintaining travel diaries or logs were discussed in Chapter 2.

During the literature review, a thorough search for articles and information on accuracy of different pedometer makes and models was conducted. This search 
concluded when one pedometer was selected for use in the study. The pedometer used in this research was manufactured by the Japanese company Yamax, and sold under the brand name 'Digi-Walker'. The Digi-Walker has a number of models; the SW-651 was used for this study. The decision to use a Yamax pedometer was based on research (Bassett, et. al., 1996) which compared five different brands of pedometers in terms of accuracy and concluded that Yamax measured number of steps to within 1\% of actual. This pedometer costs around $\$ 30$. Figure 3 shows a picture of the pedometer used for the study.

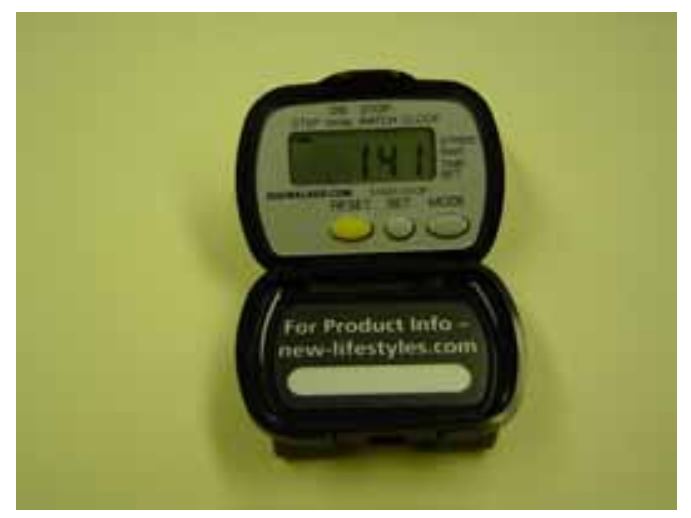

Figure 3: Yamax Digi-Walker Pedometer Used in This Study

The Yamax Digi-Walker contains a number of features. Bassett, et. al. (1996) found the unit to be excellent for reliability and accuracy in step counting. In addition to counting steps, it also gives the distance traveled in miles once the user's stride length is entered. The stride length can be set in .05 -foot increments. The unit includes a stopwatch and a clock.

As noted in the literature review, there are some concerns regarding the pedometer as a tool for transportation data collection. Issues such as effect of gradients and surface type on the number of steps are still not resolved. It is also assumed that social interaction between walkers and interference from other pedestrian traffic, affects 
stride length and, therefore, the number of steps. The experiments developed to address these issues are described later in this chapter.

\subsubsection{Hand-Held GPS Unit}

The hand-held GPS used for this research was the 'eTrex' model, manufactured by Garmin. Approximate cost of the unit is $\$ 100$. As described by the manufacturer, this device uses a 12-parallel-channel GPS receiver that continuously tracks and uses 12 satellites to compute and update the position of the user. The GPS unit is powered by two AA batteries and is capable of running for 22 hours in power save mode. The eTrex unit has a LCD display screen on the front and a built-in antenna on top as shown in Figure 4. Figure 5 shows a close-up view of the display screen. There are five user buttons located on the sides, allowing for one-handed operation that does not obstruct view of the display.

During the experiments, it was also observed that the hand-held unit was provided with a lanyard at the bottom of the device (on the opposite side of the antenna, which should point to the sky), to wear the device around the neck. This location of the lanyard was found to be in appropriate, if the device is worn on the neck with the lanyard, the antenna faces the ground. This is the opposite of the way it should be held, as the device needs to be upright with the antenna facing the sky for receiving the best satellite signal. Due to this characteristic of the device, the subjects participating in GPS experiments were asked to keep or hold the device upright with antenna in direct view of the sky. The device must be held upright in order to communicate with satellites. It was observed during the experiments that the device would not work properly if held in hand by one's side (i.e., with the top of device facing the ground). 


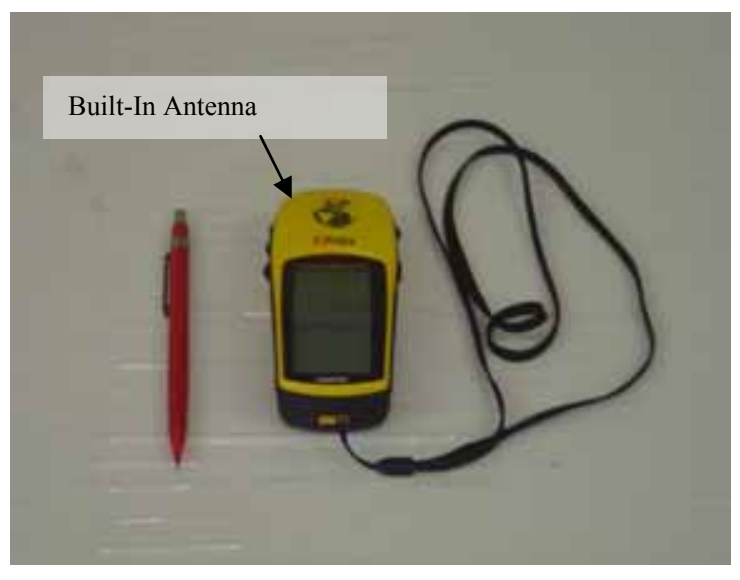

(a)

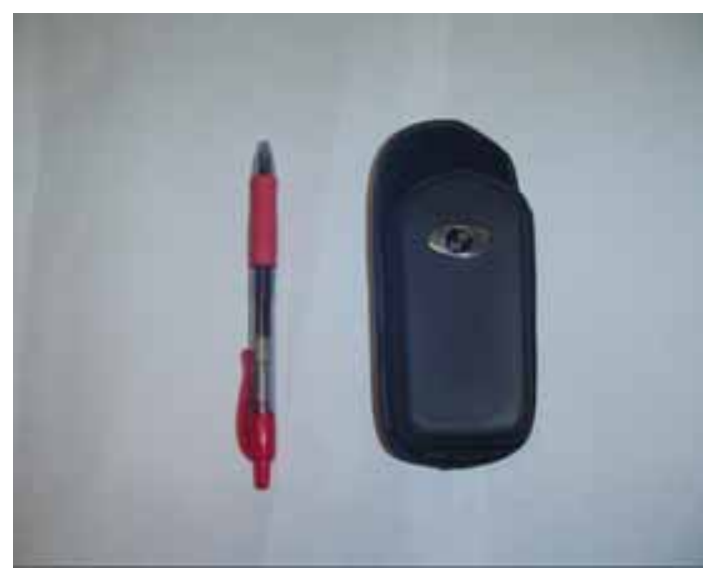

(b)

Figure 4: (a) eTrex Hand-Held GPS Device and; (b) Holster Used for This Study

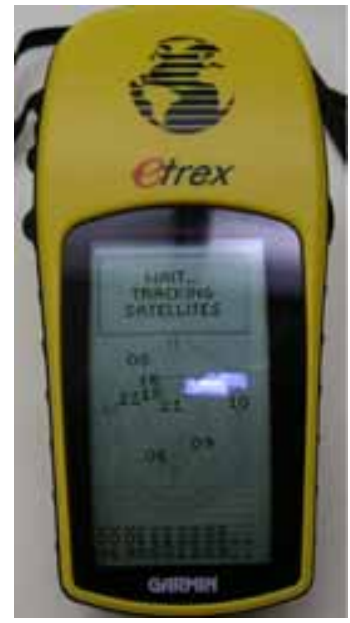

Figure 5: Close-up View of Display Screen of eTrex Hand-held GPS Device in Upright Position

In addition to determining location, the 'eTrex' creates names and saves a location (as an electronic waypoint) in its memory, allowing navigation to the saved location at any time. The eTrex keeps a continuous automatic track log of these saved locations; the log is updated every few seconds. Besides keeping a track log, the trip computer also provides the current speed, average speed, direction of movement, bearing, elevation, time of sunrise/sunset, trip timer, trip distance and reset-able maximum speed. Other features include: saving 500 waypoints with name and graphic symbol, saving 10 tracks and navigating a course of 50 waypoints in sequence. It should also be noted that 
the software in the unit, used in this research, has been superceded by newer versions of the software which have additional capabilities, such as recording and displaying 'timemoving' and 'time-stop' (which may be significant to calculate delays along a route).

The hand-held GPS unit has been used primarily for recreational purposes. No published literature on its use as a transportation data collection device was found during the literature review. This study attempted to determine the feasibility of using it as tool for collecting pedestrian travel data.

The GPS unit, although capable of collecting various travel data, is unable to collect data about trip purpose, mode of travel and trip origin and destination. In this study, such data was collected with the help of travel log forms which supplemented the GPS unit in some of the experiments.

\subsubsection{Wearable GPS Data-Logger}

Wearable GPS data-loggers can be used to collect detailed travel data for all modes including motor vehicles, walking and bicycling. The data-logger unit comes in a package which also contains a GPS receiver/antenna (located on the shoulder strap), powered by a rechargeable battery pack (also included). Figure 6 shows the components of the GPS data-logger used in the study.

The wearable GPS device used in this study was the GeoStats GeoLogger ${ }^{\mathrm{TM}}$. This data-logger can be purchased for around $\$ 900$. The GeoStats GeoLogger ${ }^{\mathrm{TM}}$ is a data capture tool that records second-by-second position and speed data based on user-defined rules. 


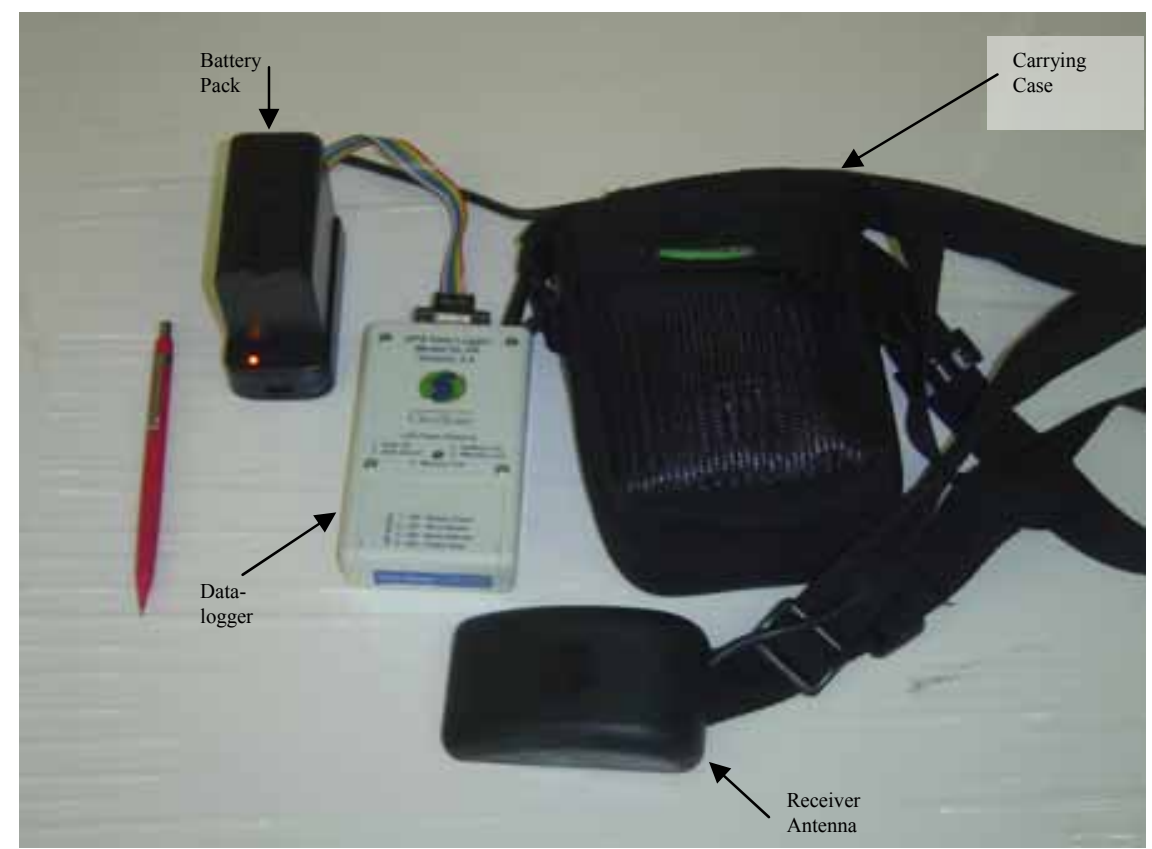

Figure 6: Components of the GPS Data Logger Used in This Study

This passive logger has no user interface and requires no user input. Figure 7 shows how it is worn by a user.

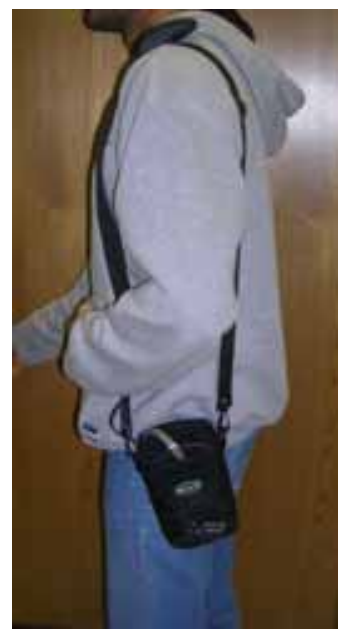

Figure 7: Wearable GPS Data-Logger Worn by a User. Note That the Antenna is Positioned on the Shoulder.

The data available from the device is the standard output data from a GPS antenna, namely:

- Latitude and longitude (degrees and decimal degrees) 
- Altitude in meters above sea level

- Heading in degrees from North

- Universal Time Coordinated (UTC) Time

- UTC Date

- $\quad$ Speed

- HDOP (Horizontal dilution of precision)

- Number of satellites in view

The logger also has different logging options:

- Record position only or record position and speed

- Record at either 1-second or 5-second intervals

- Record all valid points or record only valid points with speed greater than $1 \mathrm{mph}$

- Record altitude or not

This GPS device is capable of logging track points at a pre-specified interval, usually 1,2 or 5 seconds. It is capable of recording considerable quantities of data, which must subsequently be analyzed so as to provide information useful to transportation planners and others (Stopher, et al., 2002b).

\subsection{Evaluation of Device Characteristics}

\subsubsection{Pedometer}

As noted previously, pedometers count steps. To determine distance traveled, the number of steps must be multiplied by a stride length. However, intuitively, it would seem that an individual's stride length is not always the same, thus adversely affecting pedometer accuracy in measuring distance. Stride length could be affected by the type of surface on which one is walking. Steepness of the grade being ascended or descended 
could also affect stride length. Walking with another individual, particularly if the two are engaged in conversation would be expected to affect stride length. Similarly, encountering other pedestrians on a walkway either moving in the same or the opposite direction, would be expected to affect stride length as pedestrians may have to speed up or slow down or shift laterally to maneuver around other pedestrians. Given all of the factors that could affect stride length, there are questions about the accuracy of pedometers in measuring distance.

Four experiments were devised and conducted to evaluate the accuracy of pedometers relative to their suitability for transportation data collection:

- Effect of Surface Type on Number of Steps

- Effect of Gradient on Number of Steps

- Effect of Social Interaction on Number of Steps

- Effect of Other Pedestrian Traffic on Number of Steps

This section presents the methodology for each of the experiments. For the first two experiments, a statistical determination of sample size was desired so that differences between the categories of independent variables could be determined statistically.

A statistical formula for sample size (n) determination, frequently used in traffic engineering, is given by,

$$
n=\left[\frac{z_{z} Z^{\sigma}}{\mathrm{E}}\right]^{2}
$$

where, $\mathrm{n}=$ sample size 
$z_{\alpha / 2}=$ known as the critical value, the positive $z$ value that is at the vertical boundary for the area of $\alpha / 2$ in the right tail of the standard normal distribution (shown in Figure 8) $\sigma=$ the population standard deviation ( $\sigma$ can also be determined from a similar process or from a pilot test/simulation), and $\mathrm{E}=$ the margin of error (maximum difference between the observed sample mean $\bar{x}$ and the true value of the population mean $\mu$ ).

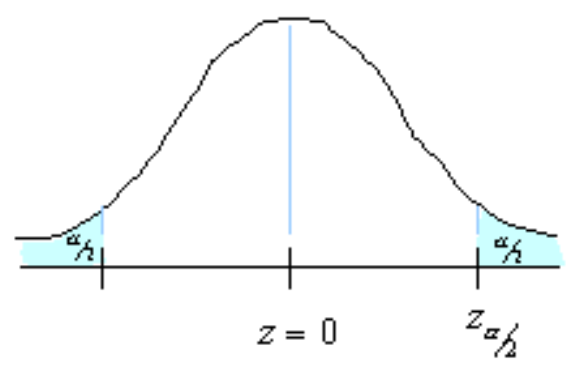

Figure 8: Standard Normal Distribution Showing the Critical Value $z_{\alpha / 2}$ in the Right Tail.

In this case, ' $n$ ' is the number of subjects to be tested. The data to be recorded for each subject is number of steps. Lacking information on the variability of number of steps, a pilot study was conducted. The standard deviation obtained from a pilot study (where 7 subjects walked a distance of 300 feet) on the number of steps needed to cover a fixed distance, conducted earlier gave $\sigma=31.15$ steps or $\approx 32$ steps. The margin of error (E) was assumed to be 10 steps. For a 95\% confidence level, $\alpha=0.05$ and $\alpha / 2=0.025$. The region to the left of $z_{\alpha / 2}$ and to the right of $z=0$ is $0.5-0.025$, or 0.475 . From a Table of the Standard Normal Distribution (Appendix A), an area of 0.475 corresponds to a value of 1.96. The critical value is therefore $z_{\alpha / 2}=1.96$. Substituting the above values in the sample size equation yields,

$$
n=\left[\frac{z_{\alpha / 2^{\sigma}}}{E}\right]^{2}=\left[\frac{1.96 \cdot 32}{10}\right]^{2}=[6.43]^{2}=41.46 \approx 42
$$


Thus, for a confidence level of $95 \%$, the sample size required is 42 . Similarly, for different confidence levels, the required sample sizes are,

Confidence level $=90 \%, \mathrm{n}=29.38 \approx 30$,

Confidence level $=85 \%, \mathrm{n}=22.38 \approx 23$,

Confidence level $=75 \%, \mathrm{n}=14.27 \approx 15$,

Confidence level $=70 \%, \mathrm{n}=11.67 \approx 12$

Since this was an unfunded study involving multiple experiments, resources were not available to compensate subjects for their participation. Sample sizes on the order of 20 to 40 individuals per experiment, while desired, were simply not feasible. A sample size of 12 was chosen for each of the first two experiments since this number of volunteer participants could be recruited without difficulty. However, it is recognized that the corresponding confidence level of $70 \%$ is less than that commonly used in research.

For the third experiment i.e., effect of social interaction on the numbers of steps, a sample size of five was chosen. Since the result expected from the experiment cannot be expressed in terms of numbers, but in terms of qualitative statement, a sample size of five was assumed to be sufficient to draw preliminary conclusions from the data collected.

\subsubsection{Effect of Surface Type on Number of Steps}

The purpose of the experiment was to determine the number of steps taken by subjects (varying in gender, age and stature) to cover a pre-determined distance on different types of walkway surfaces. The step count was made using a Yamax DigiWalker pedometer. Surface types considered for this experiment were a high-type paved (asphalt/concrete) surface, grass, an unpaved limestone sand surface and an older deteriorated sidewalk surface. The test sections were essentially flat. All sites for the 
experiment were located in Morgantown, West Virginia. Two of the sites were sections on the same multi-use trail but with different surfaces. One of the sites was a sidewalk next to Beechurst Avenue, an arterial street in Morgantown. The grass surface was part of an athletic complex near the WVU Coliseum. Figure 9 illustrates the surfaces used for the experiment.

The experiments were carried out during daylight when there was no falling precipitation. To minimize possible effects of weather, such as wind, the tests were not carried out when the wind speed was above $15 \mathrm{mph}$, as this factor may affect walking behavior. Also, no experiments were conducted when the air temperature was below $35^{\circ} \mathrm{F}$.

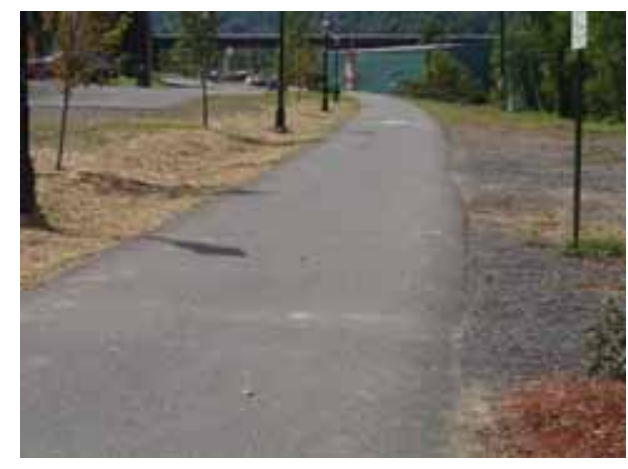

(a) High-typed paved surface

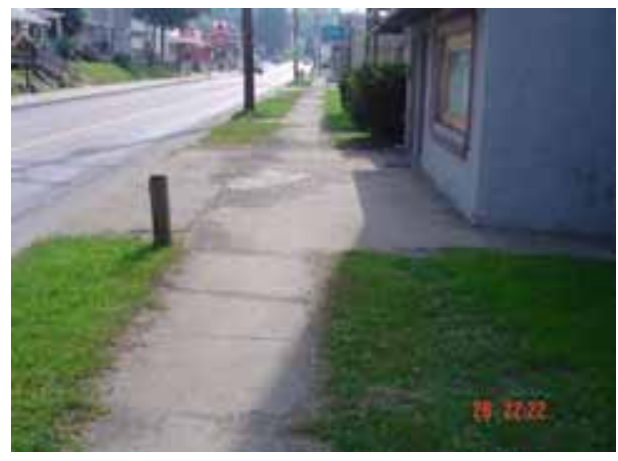

(c) Older-deteriorated surface

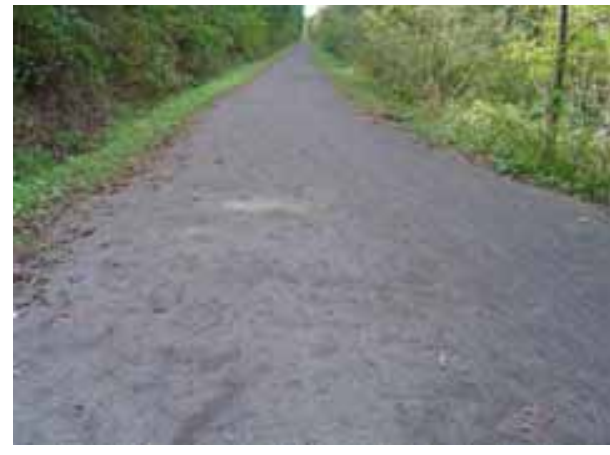

(b) Unpaved surface

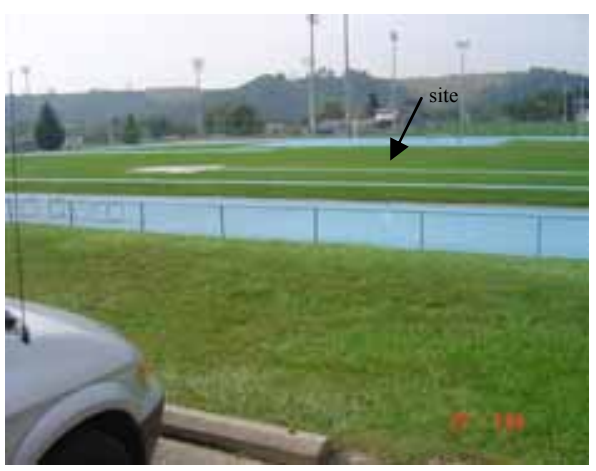

(d) Grass surface

Figure 9: Illustrations of the Types of Surfaces used in the Pedometer study

The experiments were carried out on dry surfaces only. Before each run, all atmospheric parameters were checked using current readings as posted on 
www.weather.com. Subjects wore sport shoes with rubber soles. Before starting the runs, each subject was given an instruction sheet which described the experiment and what the subjects were expected to do. The instruction sheet is contained in Appendix B.

With one exception, the subjects were all undergraduate and/or graduate students at West Virginia University. One subject was a faculty member at the University. Most of the walking in which the subjects normally engaged was traveling to/from classes. In recruiting volunteers for the experiment, the researcher made an effort to seek individuals of varying heights and stride lengths, and to involve both males and females. However, it is recognized that females are under-represented in the sample; similarly, the early 20's age group is over-represented.

For each subject, the researcher first determined the stride length. This was done by asking the subject to walk a measured distance of 60 feet wearing a pedometer. The number of steps required to cover that distance was recorded. This distance (i.e., 60 feet) was divided by the number of steps to determine the stride length. This stride length was recorded on the surface type data sheet which is included in Appendix B and used for analysis purposes. In addition to stride length, gender and height of the subject were also recorded.

After determining stride length, subjects walked on each of the measured 300-foot long sections in random order. Before starting the experiments on each of the surfaces, subjects attached the pedometer (aligned approximately in the center of the right leg) at their waist. At the start point, the pedometer was reset to zero. The subjects were then asked to walk 'normally' on each of the surfaces. At the end of test section, when the subject stopped, the researcher recorded the number of steps shown on the pedometer. 
The subject was then asked to walk back to the starting point and the procedure was repeated in the opposite direction.

The number of steps taken on each of the surfaces was recorded by the researcher on a data collection sheet, shown in Appendix B. The average of the two trials was used as the number of steps for each surface. The number of steps on grass, unpaved surface and older deteriorated surface was then compared to the number of steps on the high-type surface in order to determine the effect of surface type on step count. This procedure was repeated for all subjects.

\subsubsection{Effect of Gradient on Number of Steps}

The purpose of the experiment was to determine the number of steps taken by subjects (varying in gender, age and stature) to cover a pre-determined distance on different walkway gradients. All tests were conducted on a high-type paved (asphalt/concrete) surface. Subjects wore sport shoes with rubber soles. The step count was made using a Yamax Digi-Walker pedometer. Gradients used for this experiment were divided into four categories: $\quad 0-2 \%, 3-7 \%, 8-12 \%$ and $13-17 \%$. The sites chosen for the experiment represented each category. Gradient categories were used since, outside of the laboratory, it is difficult and time consuming to find a specific gradient, e.g., 10\%. Also, in the "real-world" it is difficult to find gradients that are uniform over several hundred feet, i.e., the gradient will change slightly over such distances. Figure 10 shows the sites used in the gradient study.

The subjects chosen were all students at West Virginia University. Among the subjects chosen for the experiment, eight of them had also participated in the 'Effect of Surface Type on Number of Steps' experiment. 


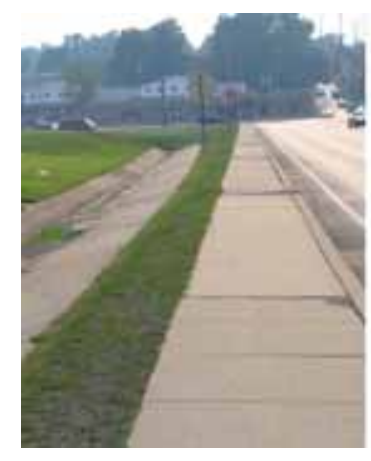

(a) $0-2 \%$ Gradient Category

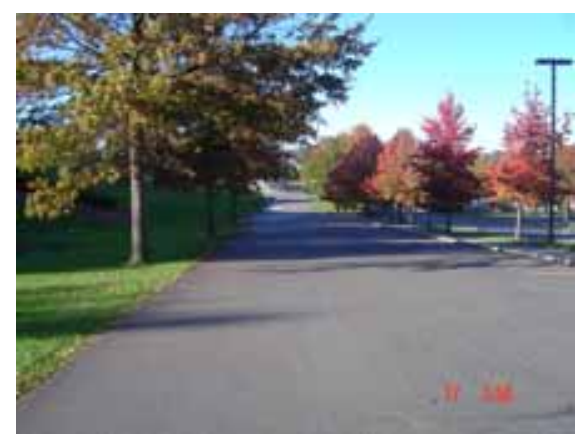

(c) $8-12 \%$ Gradient Category

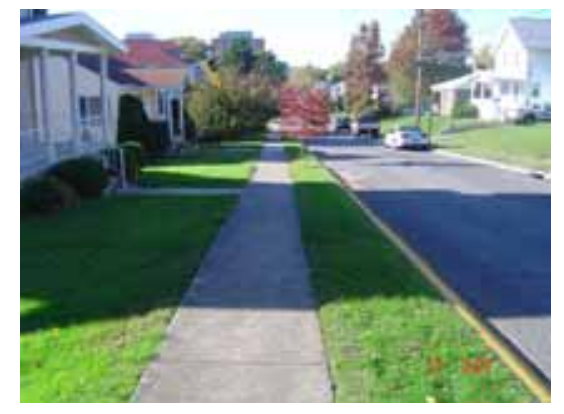

(b) 3-7\% Gradient Category

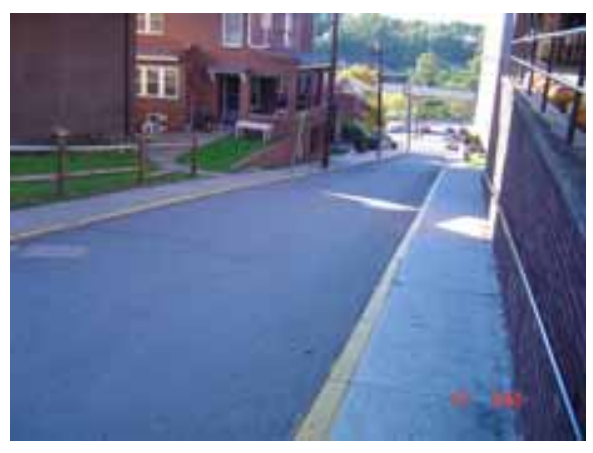

(d) $13-17 \%$ Gradient Category

Figure 10: Various Gradient Categories Used in the Pedometer Study

The experiments were carried out during daylight when there was no falling precipitation. To minimize possible effects of weather, such as wind, the tests were not carried out when the wind speed was more than $15 \mathrm{mph}$, as this factor may affect walking behavior. Also, no experiments were conducted when the air temperature was below $35^{\circ} \mathrm{F}$. The experiments were carried out on dry surfaces only. All atmospheric parameters were checked using current readings as posted on www.weather.com. Before starting the runs, each subject was given an instruction sheet which described the experiment and what the subjects were expected to do. The instruction sheet is contained in Appendix B.

The researcher first determined the stride length of the subject. This was done by asking the subject to walk a measured distance of 60 feet on the $0-2 \%$ grade surface wearing a pedometer. The number of steps required to cover that distance was recorded. 
This distance (i.e., 60 feet) was divided by the number of steps to determine the stride length. This stride length was recorded and used for analysis purposes. In addition to the stride length, gender and height of the subject were also recorded.

After determining the stride length, the subjects walked on each of the gradients in random order. Subjects walked on a measured 200-foot long section. Before starting the experiments at each of the test sites, subjects attached the pedometer (aligned approximately in the center of the right leg) at their waist. At the start point, the pedometer was reset to zero. The subject was asked to walk 'normally' on each of the gradients. At the end of the test section, when the subject stopped, the researcher recorded the number of steps shown on the pedometer. This was done once downgrade and once upgrade.

The observations of the two runs (i.e. downgrade and upgrade) were recorded by the researcher on a data collection sheet, included in Appendix B, for each gradient. The number of steps on each gradient category was then compared with the number of steps recorded on the $0-2 \%$ high-type surface to determine the effect of the gradient on step count. The procedure was repeated for each subject in the experiment.

\subsubsection{Effect of Social Interaction on Number of Steps}

This experiment was different from the two pedometer accuracy experiments just described. Even though the experiment involved subjects, it was the researcher's steps that were counted. Since the purpose of the experiment was to observe how social interaction between individuals affected stride length, the researcher recorded and analyzed step count data over a pre-determined distance for a pedestrian (the researcher) engaged in social interaction with an accompanying pedestrian (the subject). The step 
count was made using a Yamax Digi-Walker pedometer. The experiment was conducted on a level sidewalk with good surface condition. The length of the test section was 200 feet. The width of the sidewalk was 8 feet; this is more than the minimum walking width needed by two pedestrians walking side-by-side. The test section for this experiment was a length of sidewalk in front of the Engineering Sciences Building on the Evansdale Campus of WVU. All tests were done when there was no other pedestrian traffic present.

Subjects wore sport shoes with rubber soles. The subjects chosen were undergraduate and/or graduate students at West Virginia University. In this experiment, subjects were chosen on the basis of their heights. The subjects varied in height from short to tall and, consequently, the stride in lengths varied. Variability in stride length of the subjects was sought since, in conversation, the stride length of the accompanying person could affect the researcher's stride length and thus his number of steps. Before starting the runs, each subject was given an instruction sheet which described the experiment and what the subjects were expected to do. The instruction sheet is contained in Appendix B.

The experiment was conducted on a clear to partly cloudy day using the researcher as an integral part of the test. The experiment was designed to be reproducible. The researcher first determined his/her own stride length. This was done by walking the length of test-section (i.e., 200 feet) on a level, high-type surface. The number of steps required to cover the distance was recorded. This distance (i.e., 200 feet) was divided by the number of steps to determine the stride length. In a similar manner, the researcher also measured the stride length of each of the accompanying subjects. 
Along with stride length, the height of each subject was measured. This information was recorded and used for analysis purposes.

The researcher first walked alone on the test section, using the pedometer to record the number of steps. In the test runs, the researcher walked side-by-side with each subject, at a comfortable separation. For the first run, there was no conversation or eye contact and both individuals walked facing straight ahead. In the next run, the researcher walked with the same individual, but with limited talking. Limited talking refers to short conversations (for example, questions asked by the researcher or the subject that can be answered with a yes or no or merely a short reply). The talking started upon crossing a point 40 feet into the test section where a mark had been made on the surface. The last run was made with the same person; this time, there was significant conversation throughout the walk. Significant conversation here refers to a two-way conversation in which both researcher and subject were almost equally participating with more in-depth discussion than the short conversation, i.e., more than short replies. After each run, the number of steps shown on the pedometer was recorded on a data form, shown in Appendix B. Since stride length of the accompanying individual might be a factor affecting the number of steps, the test was replicated for 5 different accompanying subjects.

\subsubsection{Effect of Other Pedestrian Traffic on Number of Steps}

This experiment was different from the previously described experiments. There were no "subjects" involved in this experiment, and no experimental setup was made. The experiment was performed making use of actual pedestrian traffic on a sidewalk. 
The researcher's steps were counted in order to ascertain the effect of other pedestrians on an individual's stride length.

The purpose of the experiment was to determine the number of steps taken by an individual in the presence of varying amounts of other pedestrian traffic on a walkway. The step count was made using a Yamax Digi-Walker pedometer. The experiment was conducted by the researcher with the "other" pedestrian traffic varying from no other pedestrians to Levels of Service A to C, using the Highway Capacity Manual (TRB, 2000) approach to Pedestrian Level of Service. The experiment was conducted on essentially level sidewalks in good condition, in clear to partly cloudy weather. The researcher wore sport shoes with rubber soles.

The experiments were carried out during daylight when there was no falling precipitation. To minimize possible effects of weather, such as wind, the tests were not carried out when the wind speed was above $15 \mathrm{mph}$, as this factor may affect walking behavior. Also, no experiments were conducted when the air temperature was below $35^{\circ} \mathrm{F}$. Before each run, all atmospheric parameters were checked using current readings as posted on www.weather.com. The weather on each day of the experiment was similar, i.e., partly cloudy with temperatures varying from 58 to 73 degrees $F$.

The experiments were carried out over 7 days from mid -to late -April, 2005 on the campus of West Virginia University. The sidewalks chosen for the experiment were high-type surfaces. The sites for the experiments were the sidewalk in front of the Mineral Resources Building on the Evansdale Campus and the sidewalk on the south side of Prospect Street on the Downtown Campus of West Virginia University. These sites were chosen since they had defined sidewalk widths of between 5 and 12 feet and had 
relatively high volumes of pedestrian traffic (with respect to the campuses of West Virginia University).

The first test section was a 100-foot long section of sidewalk between the entrance to the Engineering PRT station and the Engineering shuttle bus stop. This sidewalk (Site 1) was 12 feet wide. The second test-section was the sidewalk located along Prospect Street, next to Clark Hall of Chemistry. The area of Site 1 was 1200 sq feet. The Prospect Street sidewalk (Site 2) was 6 feet wide; however, considering street furniture (parking meters, street-lighting, and kiosks) the effective width was only 5 feet. The area of Site 2 on the Downtown Campus was 500 sq ft.

For both sites, the ends of the section and the mid-point were measured and marked beforehand. All test runs were carried out during morning hours between 8a.m. and 11a.m., when the maximum pedestrian traffic occurred at the study locations.

Conduct of this test required two persons. Since the researcher was an active participant, another individual was needed to take photographs at each of the sites during the test runs. A graduate student colleague of the researcher volunteered to serve as photographer. He was positioned at an elevated vantage point, from which the entire test section could be seen. The section was photographed when the researcher was approximately in the middle of the test section. A sample photograph is shown in Figure 11. At both sites, there were few times when the pedestrian traffic was equal in both directions. 


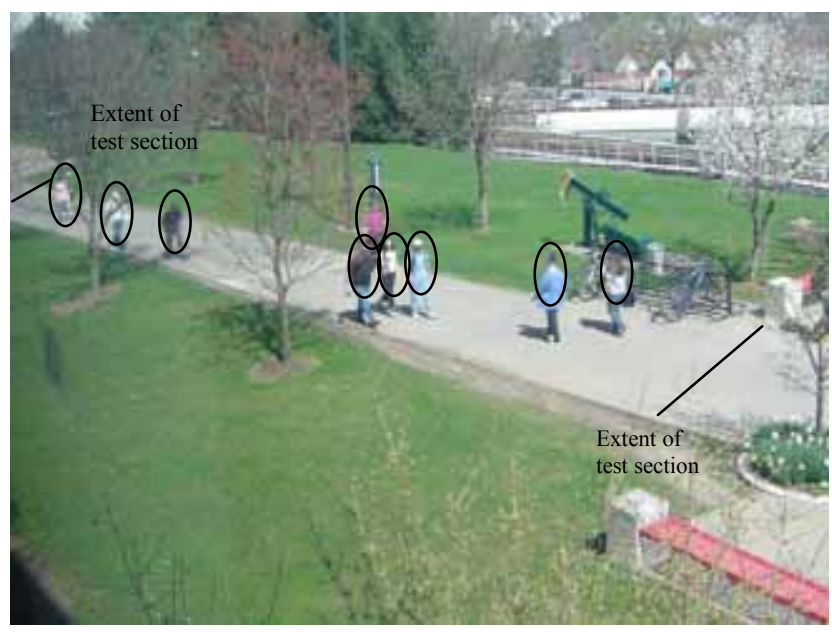

Figure 11: Photo of Site 1, from Observer's Vantage Point, Used to Calculate Level of Service

At Site 1, there was directionality to the pedestrian flows due to its location near a campus intermodal connection. Flows were in one direction when the campus bus arrived and unloaded students and were in opposite direction when the students disembarked from the public transit system. At Site 2, students generally went in one direction going to class and in the opposite direction after class. Thus test runs were carried out in both directions, i.e., with the pedestrian traffic and against the pedestrian traffic.

As noted above, no subjects were recruited for this experiment; the study was performed using the actual pedestrian traffic present on each sidewalk at that time. The experimental runs were carried out for pedestrian traffic conditions varying from Level of Service (LOS) A (pedestrian space $>60 \mathrm{ft}^{2} / \mathrm{p}$ ) to LOS C (pedestrian space between 24 and $40 \mathrm{ft}^{2} / \mathrm{p}$ ). While it would have been desirable to study all levels of service, levels of service D through $\mathrm{F}$ are usually found in Morgantown only during special events. The experiment attempted to collect data on about 5 runs for each level of service.

The researcher first determined his stride length. This was done by walking a distance of 100 feet on a level high-type surface. The number of steps required to cover 
that distance was recorded. This distance (i.e., 100 feet) was divided by the number of steps to determine the stride length. This stride length was recorded and used for analysis purposes.

The researcher wore the pedometer on the waist, at approximately the center of right leg for each test run. He walked the test section, in both directions, when no other pedestrian traffic was present. At the start of each test run, the pedometer was reset to zero. The researcher walked with the traffic and against the traffic on the test section for varying volumes of pedestrian traffic. After each run, the researcher noted the number of steps, as shown on the pedometer display, on a data sheet shown in Appendix B.

The level of service during any test run was determined after the fact, using photographs (such as Figure 11) of test section taken at the time of experimental run. This was done since it was difficult to estimate the level of service in real time simply by observing the pedestrian traffic. After numerous (approximately 50) test runs were conducted, the number of steps recorded for each run were compiled and the photographs corresponding to the runs were sequentially arranged. The photographs from each test run were arranged, and the researcher examined each photo. The number of pedestrians present in the test-section during that particular run was counted. The area (in square feet) of the test-section was then divided by the number of pedestrians counted for each test run to obtain a value for 'pedestrian space'.

Pedestrian space as described by the Highway Capacity Manual (TRB, 2000) is the average area provided or available for each pedestrian in a walkway, expressed in terms of square feet per pedestrian. The calculated pedestrian space permits determination of the level of service (LOS) for that section of the walkway as was 
described in Chapter 2. The pedestrian LOS criteria on a walkway, as described by the Highway Capacity Manual are presented in Table 1.

Table 1 - Pedestrian LOS Criteria as Given by Highway Capacity Manual (TRB 2000)

\begin{tabular}{|c|c|}
\hline Level of Service (LOS) & Pedestrian Space Criteria (sq. ft./p) \\
\hline A & $>60 \mathrm{ft}^{2} / \mathrm{p}$ \\
\hline B & $40-60 \mathrm{ft}^{2} / \mathrm{p}$ \\
\hline C & $24-40 \mathrm{ft}^{2} / \mathrm{p}$ \\
\hline D & $15-24 \mathrm{ft}^{2} / \mathrm{p}$ \\
\hline E & $8-15 \mathrm{ft}^{2} / \mathrm{p}$ \\
\hline F & $\leq 8 \mathrm{ft}^{2} / \mathrm{p}$ \\
\hline
\end{tabular}

As an example, Figure 11 shows Site 1 from the observer's vantage point. In the photograph, there are 9 pedestrians visible on the sidewalk. For ease of identification, these have been circled in Figure 11. Site 1 was 100 feet long and 12 feet wide. Therefore,

Pedestrian Space $=\frac{\text { Area of Test Section }}{\text { Number of Pedestrians }}=\frac{100 \times 12}{9}=133.33$ sq. ft/ ped.

According to Table 1, a pedestrian space of 133.33 sq. ft/ped. corresponds to Level of Service (LOS) A since it is clearly greater than the $60 \mathrm{ft}^{2} / \mathrm{p}$ criterion.

The number of steps taken for each run for different pedestrians volumes on the sidewalk by the researcher was recorded on a data collection sheet (Appendix B). The average numbers of steps for all runs at a particular LOS were then compared to the number of steps required by researcher when there was no pedestrian traffic present, in order to determine the effect of pedestrian traffic on stride length.

\subsubsection{Hand-Held GPS Unit and Wearable GPS Data-Logger}

As noted previously, GPS devices operate by communicating with satellites. It was recognized early on that certain environments may adversely affect this communication and thus limit the suitability of portable GPS device for collecting 
pedestrian data for transportation planning/engineering purposes. Environments of interest, which had been identified through the literature review and discussion with practitioners, included areas under high-voltage power lines, under dense tree canopies, between tall buildings and very heavy precipitation. Thus, experiments were designed to assess the effects of these environments on the GPS signal. Each of the four experiments is described in this section.

The process of downloading data recorded by the GPS units for analysis purposes, for all of the experiments is described here. The data obtained from the wearable datalogger was downloaded to a computer by using the lead provided with the unit and using the software $\left(\right.$ Geologger Download ${ }^{\mathrm{TM}}$ ) provided with the unit. The raw data obtained directly from the data-logger, is in the form of rows of numbers, where each row gives information such as date, time, position, speed, altitude and satellites corresponding to an individual record made during a particular time interval. These data were then saved as a text document, which was used for analysis.

For the hand-held unit, the process was similar. The hand-held GPS unit was connected to the computer using a wire to connect the unit to the Universal Serial Bus (USB) port of the computer. This wire was purchased separately and is readily available in the market. It is an RS232 Serial cable that is specifically designed for the eTrex models of Garmin GPS device and enables PC connectivity. The data recorded in the hand-held GPS unit was downloaded to the computer with the help of the software "DNR Garmin" developed by Minnesota Department of Natural Resources. The software is available on the internet and can be downloaded free of charge from the following website/URL:http://www.dnr.state.mn.us/mis/gis/tools/arcview/extensions/DNRGarmin/ 
DNRGarmin.html. These data were then saved as a comma delimited text file and used for analysis.

\subsubsection{Signal Availability Under High-Voltage Power Lines and Towers}

The purpose of this experiment was to ascertain the availability of satellite signal to the wearable GPS units under or in vicinity of high-voltage power lines and transmission line towers. To minimize possible effects of weather (precipitation and cloud cover), the experiment was conducted on a clear to partly cloudy day.

The experiment was carried out between 11:15 a.m. and 11:45 a.m. in early September, 2004, near Morgantown, in an area where power lines emanate from a coalfired power plant. Figure 12 shows a picture of the steel truss-like tower supporting the power lines, where the experiment was conducted.

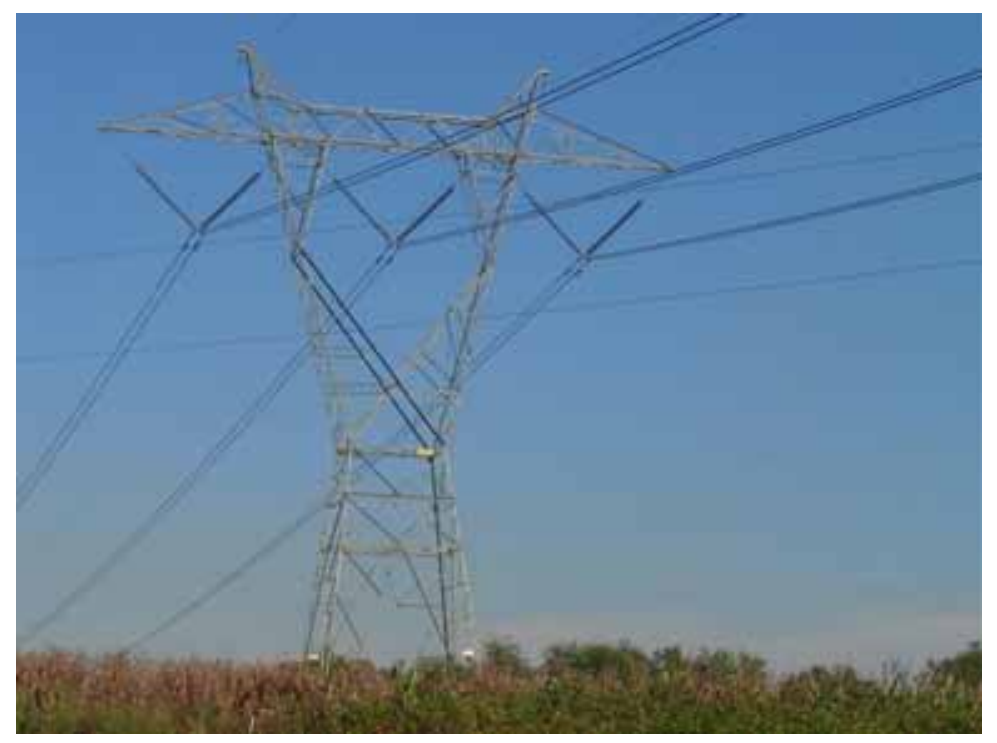

Figure 12: Photo of Site Used for Effect of High-Voltage Power Lines and Transmission Towers on Signal Availability Experiment

The GPS units were switched on as the researcher approached the power lines or their supporting transmission towers. The wearable unit was activated by simply connecting the wire from the battery pack to the data logger. Care was taken to make sure that the receiver was placed properly on the shoulder. Along with the data-logger, 
the researcher also switched on the hand-held GPS by pressing the power button. After the hand-held GPS unit showed a 'good signal' from satellites, which was evident from the signal display on the screen, the number of satellites was recorded on data sheet, shown in Appendix B. A 'good signal' refers to signals from a minimum of four satellites, which is necessary for acquiring basic data (position, altitude and time) accurately. The screen display also shows, along with the number of satellite signals, the accuracy in feet at that instant. In this case, accuracy refers to how well the position of the GPS-enabled device or the user is determined. For example, an accuracy of 20 feet means that satellites are able to locate the position of the GPS device within a radius of 20 feet. The accuracy is higher with strong signal availability and goes down with a weak signal or loss of signal. The researcher then walked to a location immediately under the high-voltage power lines, remained there for about 5 minutes, and recorded any changes at that instant in satellite signals or accuracy shown on the screen of the handheld GPS unit on the aforementioned data sheet.

The researcher then walked to a location close to a transmission line tower, waited approximately 5 minutes and again observed any changes in signal availability and the accuracy shown by the hand-held GPS unit. The number of satellite signals at different times, along with the accuracy, was noted on the data sheet. After recording the observations, the GPS units were switched off.

The data recorded by the two GPS units were then downloaded to a computer. The researcher analyzed the data to determine if there was a loss of signal during the time the experiment was carried out. This analysis was done differently for each of the two GPS units. For the hand-held GPS, a gap in the data log indicated a total loss of signal. 
Gaps were matched with observations (of signal loss) made while looking at the screen at the time of experiment. For the wearable GPS data-logger, the second column for the 'flag' indicates signal availability. A ' $\mathrm{V}$ ' indicates first valid sentence after loss of signal or power. Also, the data show the number of satellites to which the unit is connected at a particular instant. This should not be less than 4 to be considered a good signal.

\subsubsection{Signal Availability Under Dense Tree Canopy}

The purpose of this experiment was to ascertain the availability of satellite signal to the GPS units under dense tree canopy. To minimize possible effects of weather (precipitation and cloud cover), the experiment was conducted on a clear to partly cloudy day. This experiment was conducted considering both coniferous trees and deciduous trees.

A dense tree canopy refers to both individual trees and forests, where the foliage of the individual tree or trees in a wooded area allows very little sunlight to penetrate to the ground. The experiment was conducted in an arboretum and at another location where several coniferous trees were located close together. The experiment was carried out over two days in early September, 2004. The experiment was carried out in the afternoon between 2:00 p.m. and 2:30 p.m. at the first location, i.e., West Virginia University's Core Arboretum located on the Monongahela Boulevard. The arboretum includes many acres with closely spaced deciduous trees having relatively dense foliage. Figure 13(a), shows the site at the arboretum used in the effect-of-tree-canopy test.

The second location for determining the effect of dense tree canopy on signal availability was near the Creative Arts Center on the Evansdale Campus of West Virginia 
University. This location had a cluster of coniferous trees close to each other. Figure 13(b) shows the trees at the Creative Arts Center site.

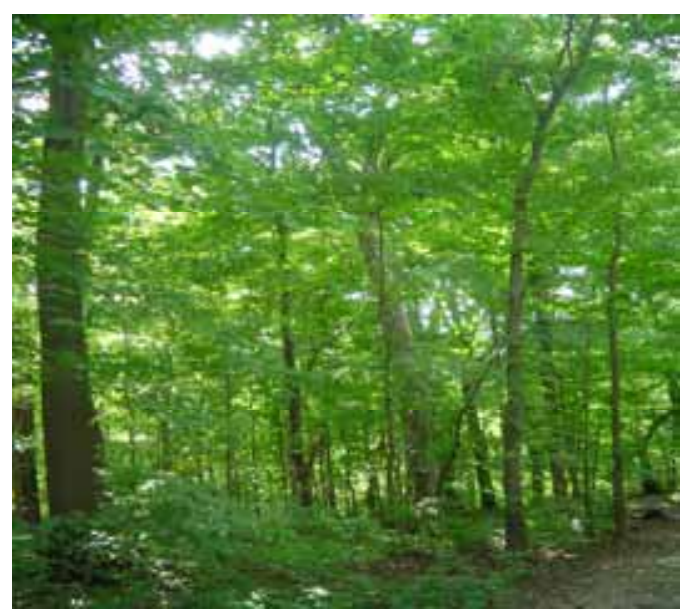

(a) Deciduous Trees in Core Arboretum

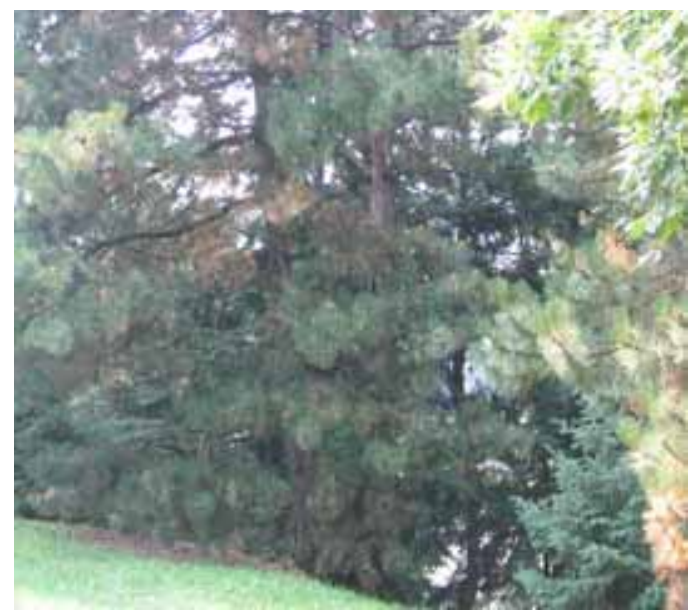

(b) Coniferous Trees

Figure 13: Sites Used for Effect of Dense Tree Canopy on Signal Availability Experiment

The GPS units were switched on as the researcher neared the two locations. The wearable unit was activated by simply connecting the wire from the battery pack to the data logger. Care was taken that the receiver was placed properly on the shoulder. Along with the wearable data-logger, the researcher also switched on the hand-held GPS by pressing the power button. After the hand-held GPS unit acquired a good signal from satellites, which was evident from the signal display on the screen, the number of satellites was recorded (using the data sheet included in Appendix B). The researcher then walked under the deciduous trees for some distance and recorded any changes in the satellite signals or the corresponding accuracy shown on the screen of the hand-held GPS unit. The researcher observed changes in the number of satellites displayed on the GPS or the accuracy while standing at a spot for about 5 minutes. Accuracy values were also recorded along with signal strength at different times on the data collection sheet shown in Appendix B. 
The researcher then determined the signal availability under the coniferous trees, which had even denser foliage, to see if the density of tree canopy made any difference. This was done by standing under the coniferous trees for about three to five minutes and observing the number of satellite signals and the accuracy (in feet) during that period. These observations were recorded on the data sheet. After the observations were recorded, the GPS units were switched off.

The data recorded by the two GPS units were then downloaded to a computer. The data were analyzed to verify if there was a loss of signal during the time the experiment was carried out. This analysis was done differently for each of the two GPS units. For the hand-held GPS, a gap in the otherwise continuous active log indicated a total loss of signal. This was compared with observations (of signal loss) that were made looking at the screen. For the wearable GPS unit, the second column for the 'flag' indicates signal availability. A ' $\mathrm{V}$ ' indicates first valid sentence after loss of signal or power.

\subsubsection{Signal Availability in Heavy Rain}

The purpose of this experiment was to ascertain the availability of satellite signal to the GPS units during heavy rain under the cover of an umbrella. The experiment was carried out in heavy rain.

Before conducting the experiment, a small pilot test was conducted to determine whether an umbrella had an effect on GPS unit performance. This was done outside, during clear weather under the shade of an umbrella to determine if the umbrella in any way affected the signal availability to the GPS units. It was determined that the umbrella had no effect on signal availability. 
The experiment was carried out in mid-afternoon in early September, on a day with heavy precipitation associated with the remnants of a tropical storm. The researcher performed the experiment by carrying the two GPS units under an umbrella between the Mineral Resources Building and the Coliseum on the Evansdale Campus of West Virginia University.

The GPS units were switched on as the researcher came outside in the rain under cover of an umbrella. The wearable unit was activated by simply connecting the wire from the battery pack to the data logger. Care was taken that the receiver was placed properly on the shoulder. Along with the data-logger, the researcher also switched on the hand-held GPS by pressing the power button. After the GPS unit acquired a 'good signal' from the satellites, which was evident from the signal display on the screen, the number of satellites was recorded (using the sample data sheet included in Appendix B).

The researcher then walked in the rain with the two GPS units under the umbrella. To minimize the effect of other factors such as buildings and trees, the researcher took care to walk in an open environment. While walking, any changes in the satellite signals or the accuracy shown on the screen of the hand-held GPS unit were recorded. The researcher observed and recorded all changes while walking for about 10 minutes. After the observations were recorded on the data sheet, the GPS units were switched off.

The data recorded by the two GPS units were then downloaded to a computer. The researcher analyzed the data to determine if there was a loss of signal during the time the experiment was carried out. This analysis was done differently for each of the two GPS units' data. For the hand-held GPS, a gap in the otherwise continuous active log showed a total loss of signal. This was compared with observations (of signal loss) that 
were made looking at the screen. For the wearable GPS unit, the second column for the 'flag' indicated signal availability or not. A ' $\mathrm{V}$ ' indicates first valid sentence after loss of signal or power.

\subsubsection{Signal Availability in the Vicinity of Tall Buildings}

The purpose of this experiment was to ascertain the availability of satellite signal to the GPS units in the vicinity of tall buildings and structures. To minimize possible effects of weather (precipitation and cloud cover), the experiments were conducted on clear to partly cloudy days.

As mentioned in the review of literature, other studies have shown that GPS devices can be adversely affected by the so-called urban canyon effect of tall buildings which are closely spaced. The satellite transmissions are evidently reflected and rereflected by these buildings much like sound echoes in a canyon. When a GPS device picks up a reflected transmission, it calculates an incorrect position. Because of this, the positions shown by the wearable unit do not represent the actual location.

For the purposes of this study, a tall building was defined as any building more than two stories high and spaced closely with respect to other structures, such as would be found in the central business district (CBD) or the downtown area of a city. The experiment was carried out at three locations, each of which had different building heights and spatial arrangements.

The first location was on the Evansdale Campus of West Virginia University between the Evansdale Library and the NRCCE Building. As shown in Figure 14, both buildings are three stories high but are only about 30 feet apart. 


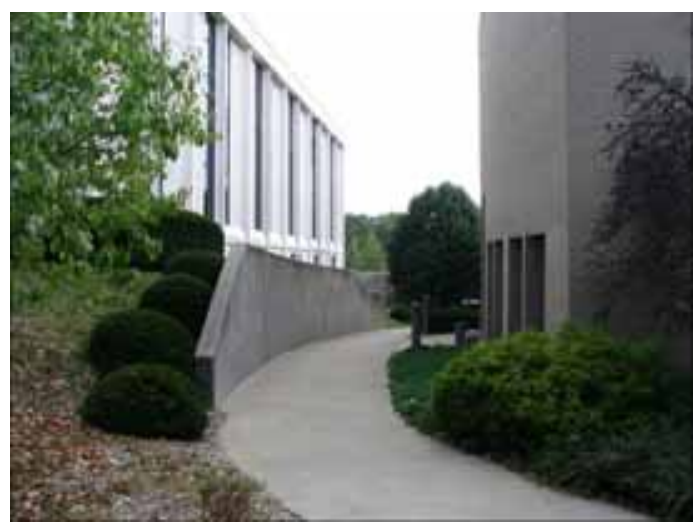

Figure 14: WVU Campus Location Used for Effect of Tall Buildings on Availability of Satellite Signal

The second location was the CBD of Morgantown, WV. Figure 15 shows that the site had three buildings, about 6 to 7 stories high on opposite sides of a narrow alley such that the total spacing between buildings was not more than 17 to 18 feet.

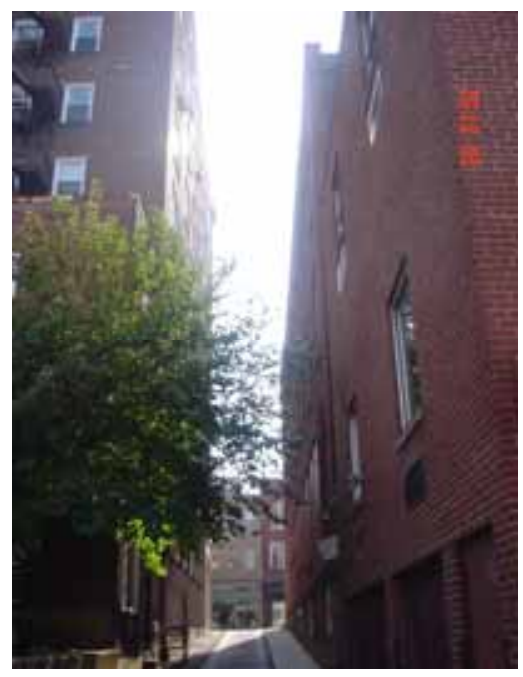

Figure 15: Downtown Morgantown Location Used for Effect of Tall Buildings on Availability of Satellite Signal

The third location was in downtown Pittsburgh, Pennsylvania and had number of tall buildings, varying in height from approximately 8 to 40 stories. Figure 16 shows a portion of the site. The exact heights of individual buildings were not recorded since the researcher walked throughout the downtown area for several blocks. Similarly, the spacing between the buildings was not recorded. It was estimated that the spacing varied from about 50 feet to 175 feet. 


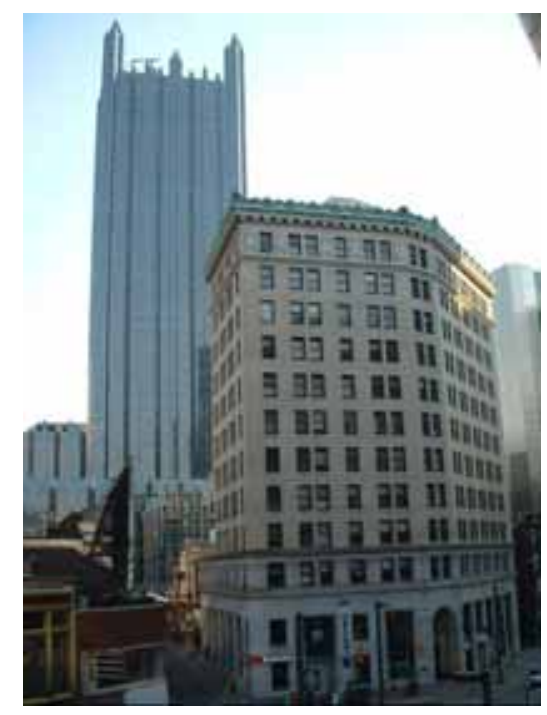

Figure 16: Downtown Pittsburgh Location, Used for Effect of Tall Buildings on Availability of Satellite Signal

In each case, the GPS units were switched on as the researcher approached the buildings in question. The wearable unit was activated by simply connecting the wire from the battery pack to the data logger. Care was taken that the receiver was placed properly on the shoulder. Along with the data-logger, the researcher also switched on the hand-held GPS by pressing the power button. After the hand-held GPS unit acquired a 'good signal' from satellites, which was evident from the signal display on the screen, the number of satellites was recorded. The recording sheet is shown in Appendix B.

The researcher then walked between the tall buildings on the sidewalk and observed and recorded any changes in the satellite signals or the corresponding accuracy shown on the screen of the hand-held GPS unit. After the observations had been recorded on the data sheet, the GPS units were switched off.

The data recorded by the two GPS units were then downloaded to a computer. The researcher analyzed the data to determine if there was a loss of signal during the time the experiment was carried out. This analysis was done differently for each of the two GPS units. For the hand-held GPS, a gap in the data log indicated a total loss of signal. 
These were matched with observations (of signal loss) made while looking at the screen at the time of experiment. For the wearable GPS unit, the second column for the 'flag' indicates signal availability. A ' $\mathrm{V}$ ' indicates first valid sentence after loss of signal or power. Also, the data shows the number of satellites to which the unit is connected at a particular instant.

\subsection{Evaluation of Operational Considerations}

The experiments carried out to evaluate the operational characteristics of the three devices are described below. These experiments were designed to assess the operational feasibility of each of the three devices with respect to collecting pedestrian data. For this category of experiments, the sample size used was five. A statistical equation was not used to determine sample size since the result expected after the analysis was not a numerical value. Rather, the result was used as input in making recommendations about whether the devices are feasible for use in transportation planning/engineering data collection. The sample sizes used in these evaluations of operational considerations seem appropriate in view of the resources available for the study. The evaluation procedure involved studying the information associated with each trip for each subject.

A 'trip' is defined as a one-way movement from a point of origin where an activity (other than travel) is being conducted to a point of destination where another activity is being conducted. Thus, an origin will be when any activity changes to a travel activity and a destination will be when the travel activity changes to any other activity. The destination location then becomes the origin for the next trip. The experiments made use a travel $\log$ form, which was given to subjects to record travel information. The form is included in Appendix C. This travel log was developed by the researcher after 
reviewing travel diary forms used in other studies, presented previously in the literature review. The travel $\log$ form was developed to be easy to use and included space for recording essential information (such as departure/arrival times and origin/destination).

\subsubsection{Pedometer}

The objective of the evaluation of operational considerations was to address various issues and assess the feasibility of using a pedometer and accompanying travel $\log$, shown in Appendix C, for collecting data on an individual's non-motorized travel. In the evaluation of operational considerations, the study was designed to address the following issues:

- Reliability

- User acceptance

- Post-processing of collected data

- Utility of the results obtained

The data collected from the experiment were analyzed to determine whether it was feasible to use a pedometer to obtain certain travel information and trip data such as trip length, origin and destination, travel speed and duration of trip.

Reliability - The data obtained from the travel log form was analyzed for both reliability of device as well as reliability of data recorded by the subject. The reliability of the device is also related to the accuracy of the device. The accuracy of the pedometer may be affected by both physical factors and human factors. Physical factors refer to such things as varying stride lengths, which may be due to other pedestrian traffic, which would affect step count. Experiments that address these issues were outlined earlier in this chapter. Human factors refer to factors such as failure to record a trip or to reset the 
pedometer, failure to wear the device properly and failure to close the device properly. Overall reliability depends on various factors. Factors such as failure to close the pedometer properly affect the step recording capabilities of the pedometer as it produces an error. Other factors include improper wearing or incorrect positioning of the pedometer on the waist, which may give erroneous step count. The analysis also tried to determine if the data recorded by the subject was reliable, i.e., whether the subjects recorded all trips and whether the pedometer was reset to zero before start of each trip.

User acceptance - The response of the subjects to wearing a pedometer and completing the trip log for all walking trips is an important input in assessing device feasibility. The analysis tried to determine if the subjects encountered problems in wearing the pedometer, in operating the device when they had to reset the step reading to zero, in reading the display to record the number of steps, and in completing the travel $\log$ for all of the trips. To evaluate user acceptance, a brief questionnaire was developed which asked questions relating to the experience of the subject. The questionnaire is included in Appendix C.

Post-processing of collected data - Since the pedometer does not have capability to record data, the only data available was the information recorded on the travel log form. These data were analyzed and post-processed to provide more meaningful and descriptive information. The time and the effort involved in doing the post-processing were also assessed.

Utility of the results obtained - The data obtained were analyzed for their utility. The experiment was conducted to assess the feasibility of using wearable technology to collect travel data and trip information for transportation planning purposes. Thus, 
results obtained from analysis of collected data from the experiment should convey the pedestrian travel data desired, directly (e.g., steps and distance) or by inference (trip purpose, origin/destination or length of trip).

\subsubsection{Use of a Pedometer for Collecting Non-Motorized Travel Data}

The pedometer was given to five subjects for a period of 24 hours. The subjects chosen were all graduate students at West Virginia University. The walking done by most of the subjects usually involved travel to and from classes. The subjects were shortlisted by considering those who, based on the researcher's knowledge, did the most walking. They were asked to wear the unit for all walking trips made in the 24-hour study period. The subjects were also given the travel log form to record certain information about each trip.

Subjects were given the pedometer at mid-day and were asked to wear the unit and record in written form, all outdoor, non-motorized travel activities (including steps) in which they were involved for the 24-hour period that followed. The subject wore the pedometer by clipping the device to the waist at approximately the center of the right leg, for the entire test period. All subjects were given an instruction sheet, included in Appendix C, prior to the experiment which described the purpose of the study and the expectations of the subjects. The subjects were instructed to take care that, if they changed clothing, or took the unit off at the end of the day, they needed to re-attach the device. They were instructed that as soon as the subject came out of a building, they needed to reset the pedometer to zero. Subjects were also instructed to take care that the pedometer was closed properly and positioned correctly and securely. In addition to the pedometer unit, each subject was also given the previously mentioned travel log form, on 
which the origin and destination and the number of steps of all outdoor walking trips made during the specified time were to be recorded. The origin/destination information was recorded in terms of a descriptive name for identification, for all individual trips. At the end of each trip, the subject also recorded the number steps shown on the pedometer display. The pedometer was to be used only for non-motorized trips made in an outside environment (as opposed to inside a building).

The subject returned the pedometer along with travel log form to the researcher 24 hours later. Subjects were then asked to complete the questionnaire about the experience. The questionnaire related mainly to user acceptance of the pedometer. The researcher then analyzed the information from the travel log form and the response to the questionnaire.

\subsubsection{GPS Devices}

The evaluation of operational considerations for the two GPS units was similar to that for the pedometer. In addition to wearing/carrying one of the two GPS units, subjects were asked to maintain an accompanying travel log for reporting certain data on their non-motorized travel. The following issues were addressed to assess the feasibility of using the GPS units and accompanying travel log for collecting data on an individual's non-motorized travel:

- Reliability

- User acceptance

- Post-processing of collected data

- Utility of the results obtained 
The data and information collected from the experiments were analyzed to determine whether it was feasible to use wearable GPS devices to collect travel data. Pedestrian travel data included trip origin and destination, speeds, route selected, trip time, delays along the trip, trip length and purpose.

Reliability - The data obtained from the GPS units, after plotting on a GIS platform, was compared to the information in the travel log and vice versa, to determine if the data were reliable. This was done to explicitly identify origins and destinations and to check if trips were recorded correctly on the travel log form. The reliability of the experiment was analyzed considering two aspects:

a) Reliability of device and data - this addressed issues such as whether the data collected was sufficiently detailed in terms of location to give the desired travel information, directly or by inference. Of particular interest was whether there was loss of signal due to effect of urban canyons, thick foliage, or other reasons.

Also, some pedestrian travel information such as speed, trip time, delay and elevations can be called non-GIS travel data since these can be determined without looking at the GIS plots of the recorded data. Normal pedestrian walking speeds usually fall between two to four miles per hour, or 0.9-1.8 meters per second range, and can be seen in the recorded data which have a separate column for subject speed. This column is present in data recorded for both the units. Trip time can be checked by looking at the beginning and the end times for the recorded data for a trip and calculating the time difference, which is confirmed from the data logger containing start and end times of a trip. The elevations can be determined by looking at the recorded data which has a separate column for elevations for data obtained from both the devices. The delay along 
the route can be determined by looking at the recorded data. In the case of the hand-held GPS unit, delay can be seen in the data recorded at instances where the speed is zero. For a wearable GPS data-logger, instances where the speed is zero but the number of satellites in contact is above 3 can be called a delay.

b) Reliability of subject - this addressed issues such as whether the subject complied with the instructions and reported all trips made and whether the subject failed to wait for the 2-minute start-up time.

User acceptance - The response of the subjects to wearing the GPS unit is an important input in assessing device feasibility. The analysis tried to address concerns such as:

o was the subject willing to carry the device along with him/her or was it thought to be a burden?

o was the subject willing to wait for 2 minutes at a location after switching on the device after leaving a building?

o did the subject forget to switch on the device?

o did the subject remember to record all origin/destination locations?

o did they provide enough information so that the trip origin/destination could be determined by the researcher?

o did they forget to record any trips they made?

o did the subject intentionally not carry the GPS unit for very short trips?

o did the GPS device or the travel log form pose any difficulty for the subject?

The data analysis also tried to address miscellaneous issues such as concerns about invasion of privacy. To evaluate user acceptance, brief questionnaires were developed 
which asked questions relating to the experience of the subject for each of the devices. The questionnaires are included in Appendix C.

Post-processing of collected data - The data collected by the hand-held GPS unit were downloaded to a computer and plotted on a map of Morgantown, with the help of a GIS software package (Arcview GIS). Hardware and software requirements and the time and effort required to do this post-processing were also assessed.

The procedure followed for the post-processing of the data collected by the two units, is briefly described here. The hand-held GPS unit was connected to the computer with the help of a cable (an RS232 Serial cable specifically designed for the eTrex models of Garmin GPS device and enables PC connectivity ) connecting the unit to the Universal Serial Bus (USB) port of the computer. This wire was purchased separately and is readily available in the market. The data recorded in the hand-held GPS unit were downloaded to the computer with the help of the software DNR Garmin developed by the Minnesota Department of Natural Resources. Once the data were received, its coordinate system was converted to North American Datum 1983 (NAD83) from the existing World Geodetic System 1984 (WGS84). This was done in order to plot the data on a street map of Morgantown which had the NAD83 coordinate system. This data was saved as a shape file $(*$.shp extension) in order to view it on ArcGIS 3.2. This process was carried out for all subjects.

For the Wearable GPS data-logger unit, the process was similar. The data obtained from the wearable GPS data-logger were downloaded to a computer by using the lead provided with the unit and using the software (Geologger Download ${ }^{\mathrm{TM}}$ ), which was provided with the unit. The raw data obtained directly from the data-logger were in 
the form of rows of numbers, where each row gives information such as date, time, position, speed, altitude and satellites corresponding to an individual record made during the time the device was "on". These data were then saved in computer memory as a text document and opened using the GPS software "DNR Garmin", developed by the Minnesota Department of Natural Resources, which helps in converting the data points from the data-logger, to a shape file. A shape file (.shp file) can be viewed with GIS software. The DNR Garmin software also converts the data points from one coordinate system to another, i.e., the coordinate system of the map on which the points are to be plotted. The original coordinate system of the raw data points was World Geodetic System 1984 (WGS84). This process of converting the coordinate system and converting data-points to a shape file was done for each of the subjects.

Once the shape file was created for each of the subject, the software Arc GIS Version 3.2, was used to view the points on a street map of Morgantown. This map was mainly a campus map (obtained from the West Virginia University's GIS Technology Center), which showed the various campuses of West Virginia University in Morgantown and details of streets in the vicinity of the campuses. Each data point was identified by a unique table of information which provided various details such as record number, position in terms of latitude and longitude, altitude, time and speed.

Utility of the results - The data obtained were analyzed for their utility. The experiment was being conducted to assess the feasibility of using wearable technology to collect travel data and trip information for transportation planning purposes. Thus, results obtained from analysis of collected data from the experiment should convey the pedestrian travel data desired, directly (e.g., speed and time) or by inference (trip 
purpose, origin/destination or length of trip). Pedestrian travel data included trip origin and destination, speeds, route selected, trip time, delays along the trip, trip length and purpose.

\subsubsection{Use of Hand-Held GPS Device for Collecting Non-Motorized Travel Data}

The hand-held GPS device was given to five subjects for a period of 24 hours. The subjects chosen were all graduate students at West Virginia University. The subjects chosen for this experiment were different from those who had participated in the operational experiments for other two devices, in order to avoid bias due to any learning effect. The walking done by most of the subjects usually involved travel to and from classes. The subjects were short-listed by considering those who, based on the researcher's knowledge, did the most walking. They were asked to carry the unit for all outdoor walking trips. The subjects were also given a travel log form to record certain information about each trip.

Subjects were given the hand-held GPS unit at noon and were asked to carry the device and record, in written form, all non-motorized travel activities in which they were involved outdoors for the 24-hour period that followed. Instructions were given to the subjects to carry the unit either in hand or in the holster (provided) fastened to their arm or backpack. The instruction sheet for subjects is included in Appendix C. As soon as the subject came out of a building, they were supposed to switch on the GPS unit by pressing the power button on the side. The GPS unit was to be held in a vertical upright position and not covered by any clothing. After switching on the unit, the subject was instructed to remain stationary at a point for approximately two minutes, to allow the GPS device to obtain a signal from the satellites and determine its position. The display 
screen on the GPS unit showed the number and strength of signal from satellites to which it was trying to connect. The subjects were instructed to resume movement towards their destination once the message 'Ready to navigate' was displayed on the screen.

In addition to the GPS device, each subject was also given the aforementioned travel log form (as shown in Appendix B) on which the origin and destination of all trips and time at respective locations, including motorized travel, were to be recorded. The subjects were instructed to switch on the GPS device only for walking trips made outside, during the specified period. For all trips made by other modes of travel, such as car or transit, only a travel log record was made on the form provided. The origin/destination information was recorded in terms of a descriptive name for identification, for all individual trips. In this manner, the subject was to record all walking trips made outside in the 24-hour study period.

The subject returned the GPS unit along with travel log form to the researcher the next day at noon. The subjects were then asked to complete the brief questionnaire about the experience. The questionnaire related mainly to user acceptance of the GPS device. The researcher then analyzed the data downloaded from the hand-held GPS, information from travel log form, and responses to the subject questionnaire.

The data downloaded from the hand-held GPS device was post-processed and plotted on a GIS platform (as described earlier). The post - processing of data was done to determine whether various types of travel information and trip data such as, nature of trip, route selection, trip length, trip times, speeds, delays en route and locations of origin and destination could be reliably and efficiently determined using GPS and/or GIS 


\subsubsection{Use of Wearable GPS Data-Logger Unit for Collecting Non-Motorized Travel Data}

The wearable GPS data logger unit was given to five subjects for a period of 24 hours. The subjects chosen were all undergraduate or graduate students at West Virginia University. The subjects chosen for this experiment were different from those who had participated in the operational experiments for other two devices, in order to avoid bias due to any learning effect. The walking done by most of the subjects usually involved traveling to and from classes. The subjects were short-listed by considering those who, based on the researcher's knowledge, did the most walking. They were asked to carry the unit for all walking trips made outdoors. The subjects were also given a travel log form to record certain information about each trip.

Subjects were given the GPS data logger at noon and were asked to wear the unit and record, in written form, all non-motorized travel activities in which they were involved outdoors for the 24-hour period that followed. The subjects were given an instruction sheet that described the experimental protocols (included in Appendix C). Subjects were instructed that as soon as they came out of a building, they were to switch on the GPS unit by connecting the wire from the data-logger to the battery. Subjects were also instructed that the receiver antenna was to be placed upright on the shoulder and was not to be covered by any clothing. After switching on the data-logger, the subject was instructed to remain stationary at a point for approximately two minutes, to allow the GPS device to obtain a signal from the satellites and determine its position.

In addition to the GPS unit, each subject was also given the previously mentioned travel log form on which the origin and destination of all trips and respective time at the 
location, including motorized travel, were to be recorded. The subjects were to switch on the GPS only for walking trips made outside, during the specified period. For all trips made by other modes, such as car or transit, only a travel log record was made on the form provided. The origin/destination information was recorded in terms of a descriptive name for identification, for all individual trips. In this manner, subjects were supposed to record all walking trips made outside in the 24-hour study period.

The subject returned the GPS unit along with travel log form to the researcher the next day at noon. Subjects were then asked to complete the brief questionnaire about the experience. The questionnaire related mainly to user acceptance of the GPS device. The researcher then analyzed the data downloaded from the data-logger, information from travel $\log$ form, and responses to the subject questionnaire.

The data downloaded from the data-logger was post-processed and plotted on a GIS platform (as described earlier). The post-processing of data was done to determine whether various types of travel information and trip data such as, nature of trip, route selection, trip lengths, speeds, delays en route and locations of origin and destination could be reliably and efficiently determined using GPS and/or GIS. 


\section{Chapter 4}

\subsection{DATA ANALYSIS AND RESULTS}

This chapter presents the data collected and describes the analysis of the data obtained from various experiments conducted. Results for pedometers are presented first followed by the results for the GPS devices.

\subsection{Evaluation of Device Characteristics - Pedometers}

\subsubsection{Effect of Surface Type on Number of Steps}

The experiment was conducted over three days in late November, 2004 using the procedure described in Chapter 3. Twelve subjects, primarily male graduate students, volunteered for the experiment. All subjects complied with the protocol presented in the previous chapter. Table 2 presents the subject characteristics.

Table 2 - Characteristics of Subjects in the Effect of Surface Type on Number of Steps Exp
\begin{tabular}{|c|c|c|c|c|}
\hline Subject & Height & Age & Gender & $\begin{array}{c}\text { Stride } \\
\text { Length (ft) }\end{array}$ \\
\hline 1 & $5^{\prime} 10^{\prime \prime}$ & 23 & M & 2.2 \\
\hline 2 & $5^{\prime} 8^{\prime \prime}$ & 24 & M & 2.45 \\
\hline 3 & $6^{\prime} 1^{\prime \prime}$ & 18 & M & 2.6 \\
\hline 4 & $5^{\prime} 5^{\prime \prime}$ & 24 & M & 2.25 \\
\hline 5 & $6^{\prime} 00^{\prime \prime}$ & 24 & M & 2.5 \\
\hline 6 & $5^{\prime} 3^{\prime \prime}$ & 24 & F & 2.05 \\
\hline 7 & $5^{\prime} 11^{\prime \prime}$ & 24 & M & 2.55 \\
\hline 8 & $5^{\prime} 9^{\prime \prime}$ & 26 & M & 2.35 \\
\hline 9 & $5^{\prime} 8^{\prime \prime}$ & 23 & M & 2.4 \\
\hline 10 & $5^{\prime} 4^{\prime \prime}$ & 24 & M & 2.05 \\
\hline 11 & $5^{\prime} 4^{\prime \prime}$ & 24 & F & 2.6 \\
\hline 12 & $5^{\prime} 7^{\prime \prime}$ & 55 & M & 2.65 \\
\hline
\end{tabular}

Approximately $85 \%$ of the subjects were male. Subjects varied in age from 18 to 55 years with a mean of 26 years. Subjects varied in height from 5'3' to 6'1', with a 
mean of $5^{\prime} 7.8^{\prime \prime}$. Stride length, measured on a high-type surface, varied from 2.05 feet to 2.65 feet with a mean of 2.38 feet.

The weather on each of the days of experiment was similar and tests were carried out during daylight when there was no falling precipitation (partly cloudy with temperatures varying from $55 \mathrm{~F}$ to $62 \mathrm{~F}$ ). Due to light rains the day before the experiment started, the grass surface (really the underlying soil) had higher moisture content than desired. Also, a small puddle was present on a section of the older deteriorated surface. All subjects performed the experiment in presence of the researcher in the manner described in the procedure. The number of steps required by each subject for each of two repetitions was recorded on a spreadsheet for analysis. The average of the two values was used for analysis purposes. The standard deviation of steps was also calculated for each surface. Table 3 presents a summary of the average number of steps and standard deviations for the surface type experiment.

Table 3 - Average Number of Steps for Each Surface Type

\begin{tabular}{|c|c|c|c|c|}
\cline { 2 - 5 } \multicolumn{1}{c|}{} & \multicolumn{4}{c|}{ Average Number of Steps on } \\
\hline & High-Type & Grass & Unpaved & $\begin{array}{c}\text { Older- } \\
\text { Deteriorated }\end{array}$ \\
\hline Subject & & & & \\
\hline $\mathbf{1}$ & 129.5 & 136 & 122.5 & 123 \\
\hline $\mathbf{2}$ & 134 & 127 & 135 & 118.5 \\
\hline $\mathbf{3}$ & 113.5 & 124 & 115 & 113.5 \\
\hline $\mathbf{4}$ & 130 & 132 & 129.5 & 127.5 \\
\hline $\mathbf{5}$ & 120 & 120.5 & 115 & 114 \\
\hline $\mathbf{6}$ & 133 & 139 & 133 & 126.5 \\
\hline $\mathbf{7}$ & 122 & 125 & 119.5 & 121.5 \\
\hline $\mathbf{8}$ & 125.5 & 125.5 & 120.5 & 122 \\
\hline $\mathbf{9}$ & 115.5 & 112 & 118 & 116 \\
\hline $\mathbf{1 0}$ & 143 & 141 & 153 & 141 \\
\hline $\mathbf{1 1}$ & 115.5 & 115.5 & 116.5 & 118.75 \\
\hline $\mathbf{1 2}$ & 112 & 112.5 & 111 & 109 \\
\hline Average & 124.46 & 125.83 & 124.04 & 120.94 \\
\hline Std. Dev. & 9.66 & 9.79 & 11.80 & 8.33 \\
\hline
\end{tabular}


From Table 2, it can be observed that most of the subjects took a similar number of steps on the high-type and the unpaved surfaces, except for subjects 1 and 10 for whom the variation was 7 and 10 steps, respectively. Almost all subjects took fewer steps on the older-deteriorated surface in comparison to the number of steps on other surfaces. This may be due to subjects taking longer strides to avoid cracks and areas of deteriorated surface and to avoid the puddle of water. Most of the subjects took slightly more steps on the grass surface in comparison to other surfaces.

Figure 17 show a graphical representation of the average number of steps. The number of steps required to cover the 300 -foot distance on each of the four surfaces was remarkably similar. Only the older-deteriorated surface varied from the others by more than two steps. As noted above, this could be due to subjects taking longer steps to step over cracks or the puddle of water.

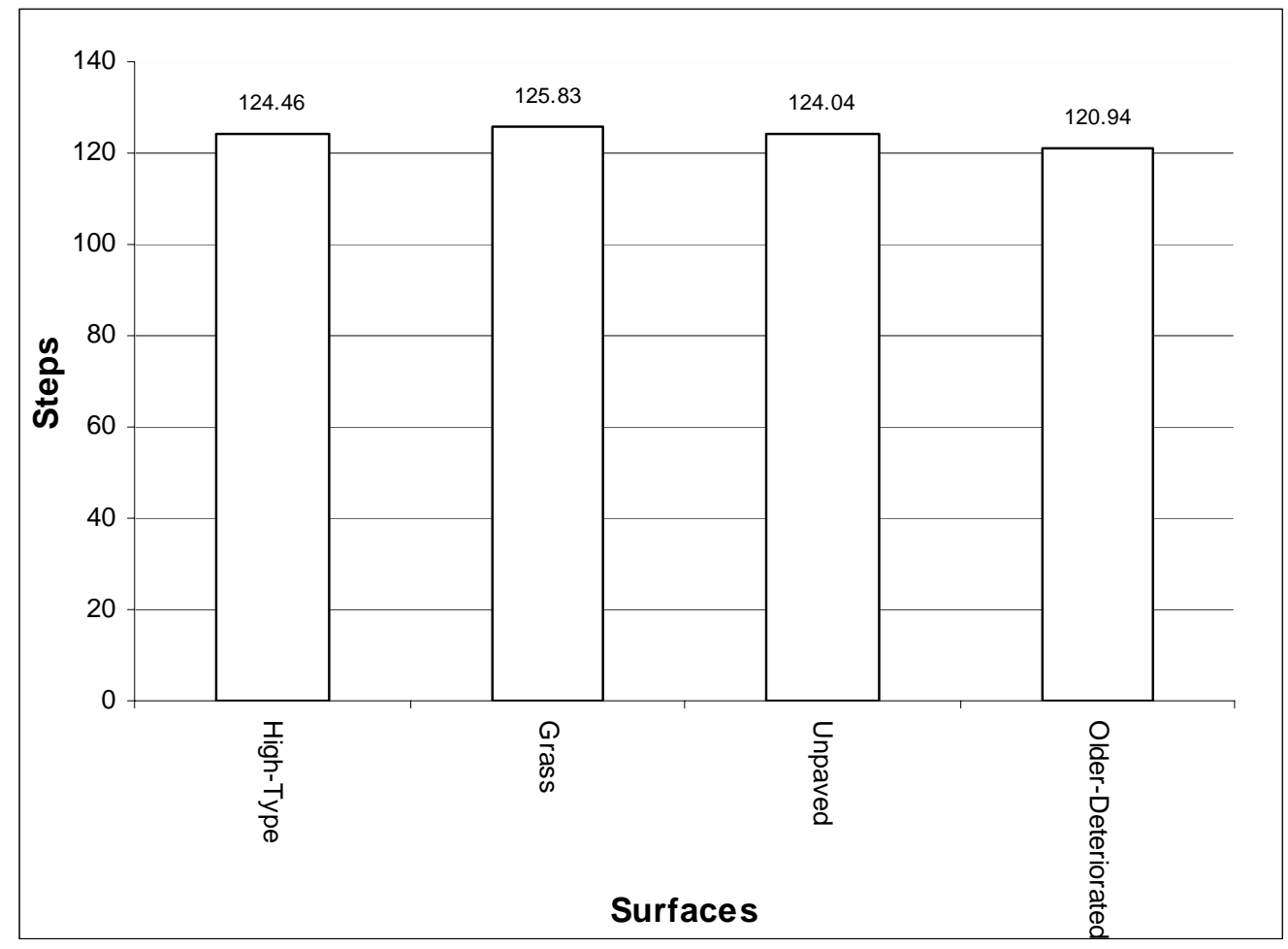

Figure 17: Average Number of Steps By Surface Type 
Figure 18 represents the plot for standard deviation in the number of steps. It can be seen that value for standard deviation varies from approximately 8 to 12 steps between the different surfaces. The standard deviation is higher for the unpaved surface while lower for older-deteriorated sidewalk. This differs from what was expected intuitively as it was assumed that the standard deviation in number of steps would be lower for the unpaved surface (which was, in fact, similar to a high-type surface), and higher for the older-deteriorated surface since it was expected that different subjects would traverse the deteriorated test-section differently.

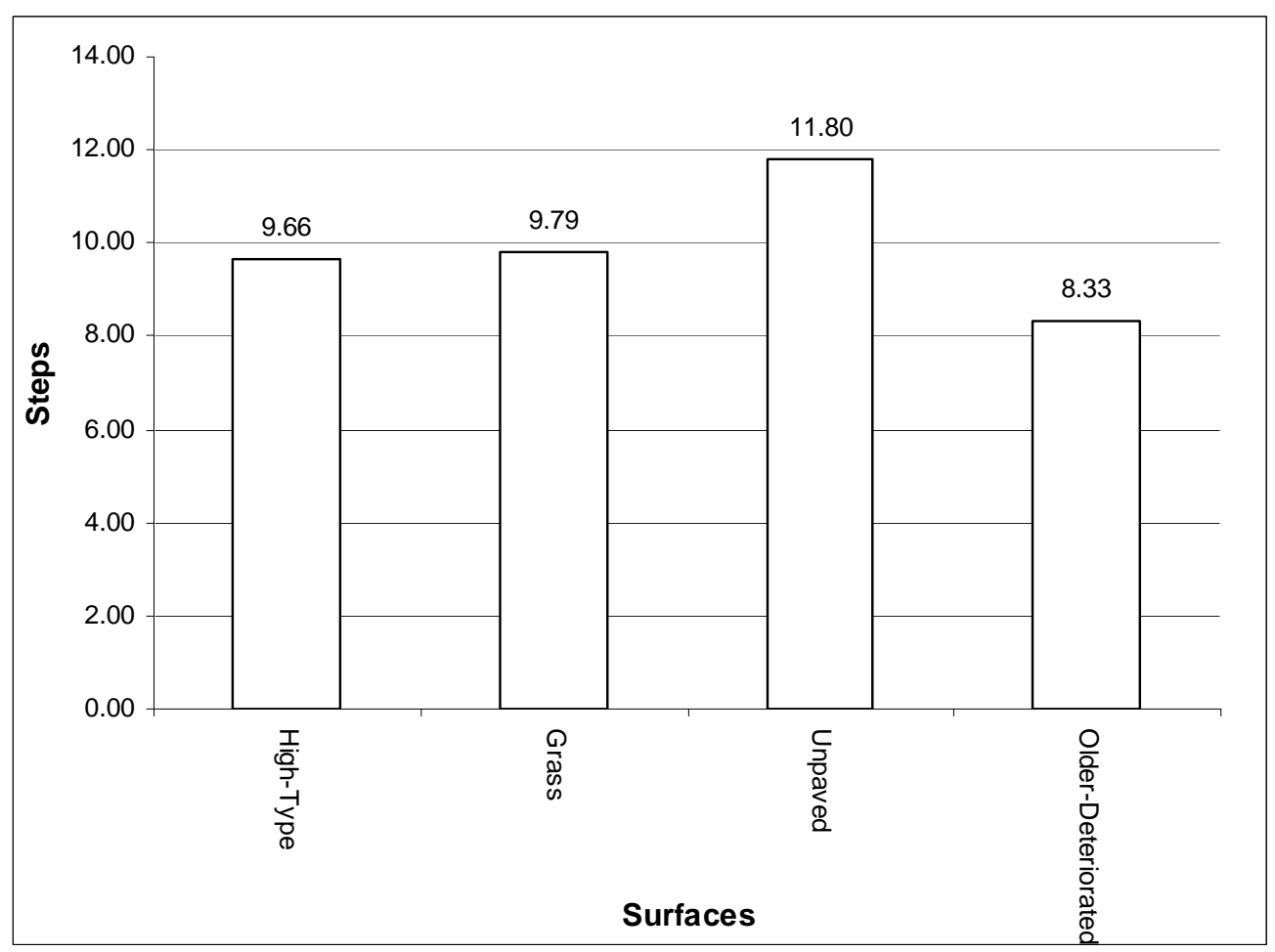

Figure 18: Standard Deviation of Steps By Surface Type

T-tests were performed between surfaces (assuming equal variance) to determine whether they are significantly different in terms of number of steps. The hypothesis is shown below, 
Null Hypothesis, $\mathrm{H}_{0}$ : Number of steps on Surface 1 = Number of steps on Surface 2

Alternate Hypothesis, $\mathrm{H}_{1}$ : Number of steps on Surface $1 \neq$ Number of steps on Surface 2

Results are shown in Table 4 . Table 4 presents a summary of the P-values obtained from t-test ( $95 \%$ confidence level) for the different surfaces. The P-value is the level of significance for the acceptance or rejection of the hypothesis. Since $95 \%$ confidence has been assumed, any P-value more than 0.05 would lead to failure to reject the null hypothesis.

Table 4 - P-Values Obtained from Comparison of Steps on Different Surfaces

\begin{tabular}{|c|c|}
\hline Surface & $\mathbf{P}(\mathbf{T}<=\mathbf{t})$ \\
\hline High-type vs. Grass & 0.73243 \\
\hline Grass vs. Unpaved & 0.68953 \\
\hline Unpaved vs. Older deteriorated & 0.46443 \\
\hline High-type vs. Unpaved & 0.92545 \\
\hline High-type vs. Older deteriorated & 0.34941 \\
\hline Grass vs. Older-deteriorated & 0.20065 \\
\hline
\end{tabular}

It can be seen from Table 4, that the P-values for each comparison were considerably higher than 0.05 (below which the null hypothesis would be rejected). It was inferred from the P-values that the null hypothesis (i.e., Number of Steps on Surface $1=$ Number of Steps on Surface 2) could not be rejected. Thus, it was concluded that there was no significant difference between numbers of steps required on the different surfaces.

This conclusion was not what was intuitively expected. This result may be due to a several factors. One of the factors may be the "under-observation effect", also known as the Hawthorne Effect. This effect refers to the alteration of human behavior because subjects know they are being studied. This effect was first demonstrated in a research project at the Hawthorne Plant of Western Electric Company in Cicero, Illinois (TRB Special Report 282, 2005). This effect can also be described as - "An experimental 
effect in the direction expected but not for the reason expected; i.e. a significant positive effect that turns out to have no causal basis in the theoretical motivation for the intervention, but is apparently due to the effect on the participants of knowing themselves to be studied in connection with the outcomes measured" (Draper, 2002).

Another possible factor is the small sample size which was not representative of the larger population. Recall that subjects who volunteered for the experiment were mostly male graduate students between 24 and 26 years of age. Thus, the subject pool was biased towards one small segment of population.

\subsubsection{Effect of Gradient on Number of Steps}

The experiment was conducted over three days in late November, 2004. As described in Chapter 3, the sites chosen for the experiment were high-type surfaces that represented each of the desired gradient categories. At each site, a 200-foot long test section was marked beforehand. Twelve subjects, primarily male graduate students, volunteered for the experiment. All subjects complied with the protocol presented in the previous chapter. Among the 12 subjects, 8 had participated in the 'Effect of Surface Type on Number of Steps' experiment.

Table 5 presents the subject characteristics. Approximately $85 \%$ of the subjects were males. Subjects varied in age from 18 to 26 years with a mean of 23.5 years. Subjects varied in height from 5 ' 4 " to 6' 1 " with a mean of 5'8.5'. The subjects were taken individually to each of the sites in random order after their stride length was measured on the flat $(0-2 \%)$ surface. Stride length varied from 2.05 to 2.7 feet with a mean of 2.48 feet. 
Table 5 - Characteristics of Subjects in the Effect of Gradient on Number of Steps Experiment

\begin{tabular}{|c|c|c|c|c|}
\hline Subject & Height & Age & Gender & $\begin{array}{c}\text { Stride Length } \\
\text { (ft.) }\end{array}$ \\
\hline 1 & $5^{\prime} 11^{\prime \prime}$ & 24 & $\mathrm{M}$ & 2.55 \\
\hline 2 & $5^{\prime} 5^{\prime \prime}$ & 24 & $\mathrm{~F}$ & 2.55 \\
\hline 3 & $5^{\prime} 4^{\prime \prime}$ & 24 & $\mathrm{~F}$ & 2.65 \\
\hline 4 & $5^{\prime} 9^{\prime \prime}$ & 23 & $\mathrm{M}$ & 2.5 \\
\hline 5 & $5^{\prime} 8^{\prime \prime}$ & 23 & $\mathrm{M}$ & 2.5 \\
\hline 6 & $6^{\prime} 1^{\prime \prime}$ & 25 & $\mathrm{M}$ & 2.6 \\
\hline 7 & $5^{\prime} 4^{\prime \prime}$ & 24 & $\mathrm{M}$ & 2.05 \\
\hline 8 & $5^{\prime} 8^{\prime \prime}$ & 24 & $\mathrm{M}$ & 2.5 \\
\hline 9 & $5^{\prime} 10^{\prime \prime}$ & 23 & $\mathrm{M}$ & 2.15 \\
\hline 10 & $5^{\prime} 9^{\prime \prime}$ & 24 & $\mathrm{M}$ & 2.7 \\
\hline 11 & $5^{\prime} 9^{\prime \prime}$ & 26 & $\mathrm{M}$ & 2.35 \\
\hline 12 & $6^{\prime} 1^{\prime \prime}$ & 18 & $\mathrm{M}$ & 2.6 \\
\hline
\end{tabular}

The weather on each of the days of the experiment was similar and tests were carried out during daylight when there was no falling precipitation (partly cloudy with temperatures varying from $44 \mathrm{~F}$ to $57 \mathrm{~F}$ ). All subjects performed the experiment in presence of the researcher in the manner described in the procedure.

The steps shown on the pedometer (Yamax Digiwalker SW651) were recorded on the data sheet after the subjects completed walking upgrade and downgrade on each gradient section. After all subjects completed the test runs, the data were grouped together on a spreadsheet and plotted for analysis. The runs were divided in two classifications, i.e., Upgrade and Downgrade for each of the gradient categories. Table 5 presents a summary of the average steps and standard deviation obtained.

Table 6 shows that for all gradient classes, whether upgrade or downgrade, the average numbers of steps was rather uniform, with a variation of less than three steps. In fact, there is almost no difference in average step count upgrade, when comparing the level surface $(0-2 \%$ grade $)$ to the steeper grade categories. 
Table 6 - Data for Each Gradient Class Collected From 12 Subjects

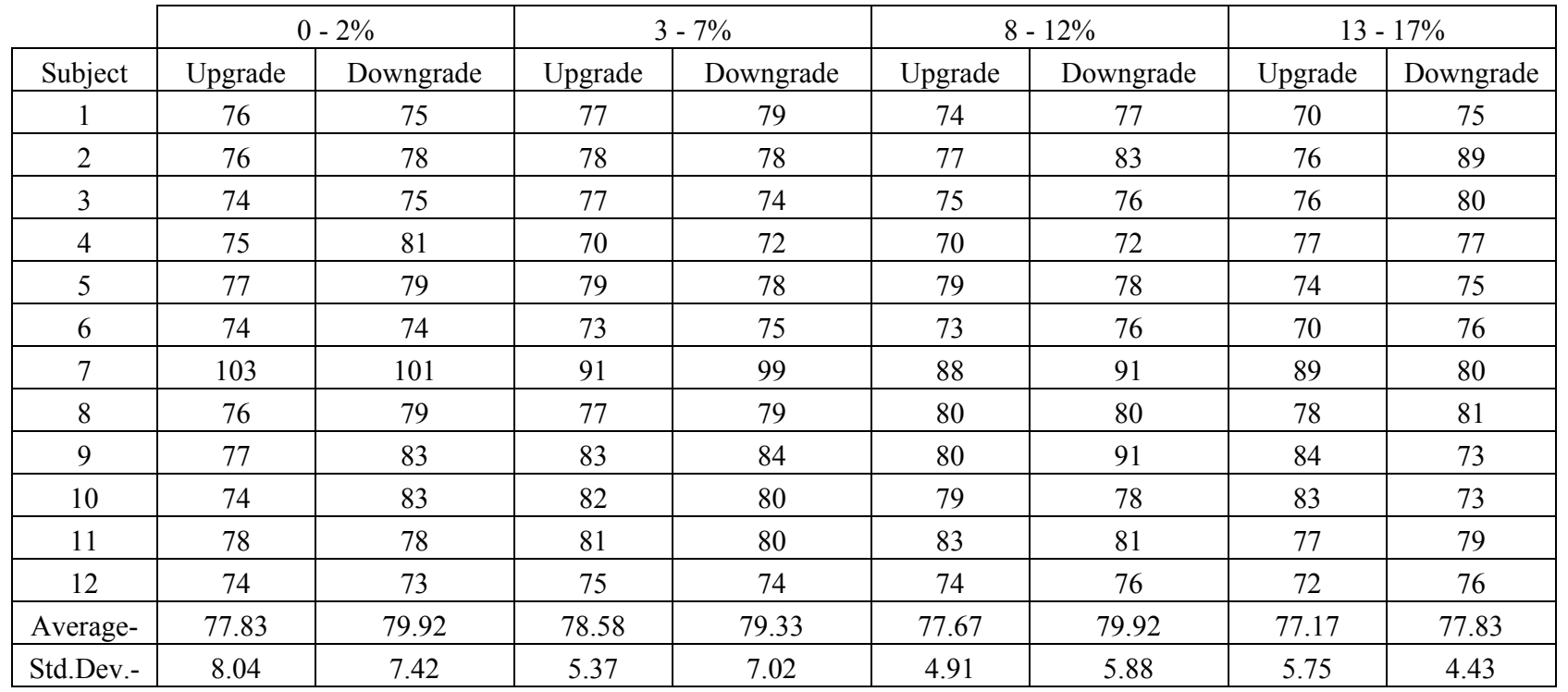

Figure 19 shows graphically the average number of steps required by the subjects, both upgrade and downgrade for each of the gradient classes.

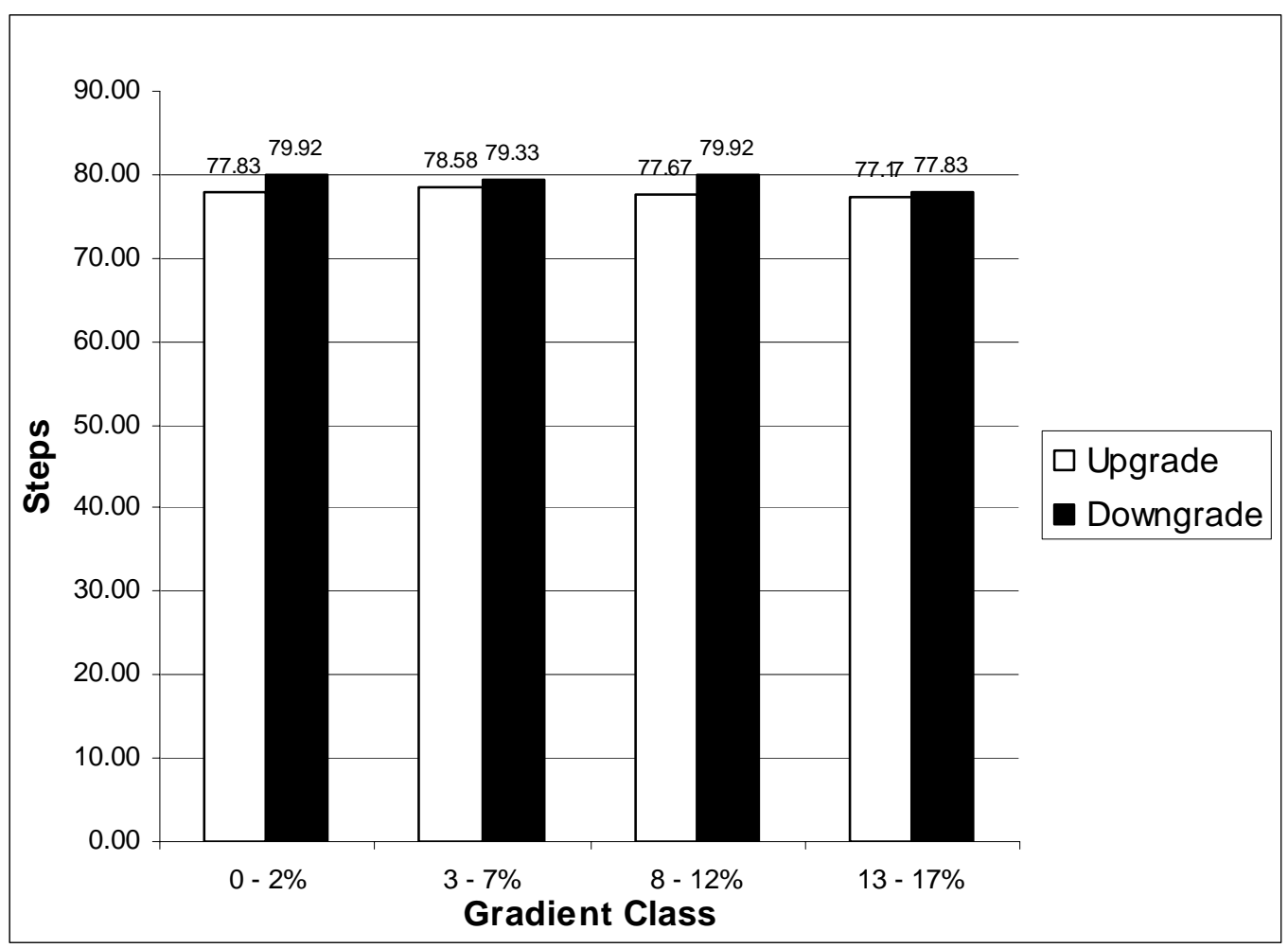

Figure 19: Average Number of Steps Required for Each Gradient Class 
This shows clearly that number of steps required to cover the 200 -foot distance, upgrade and downgrade, are about the same for each gradient class. This observation was unexpected since it was felt intuitively that stride length would change as gradient became steeper and that there would be a difference in stride length between upgrades and downgrades.

Figure 20 compares the standard deviation in the step count for both upgrade and downgrade, for each gradient class. There was no apparent trend in standard deviation for the upgrade condition. However, it was noteworthy that the near-level surface had the highest standard deviation. This was certainly not expected.

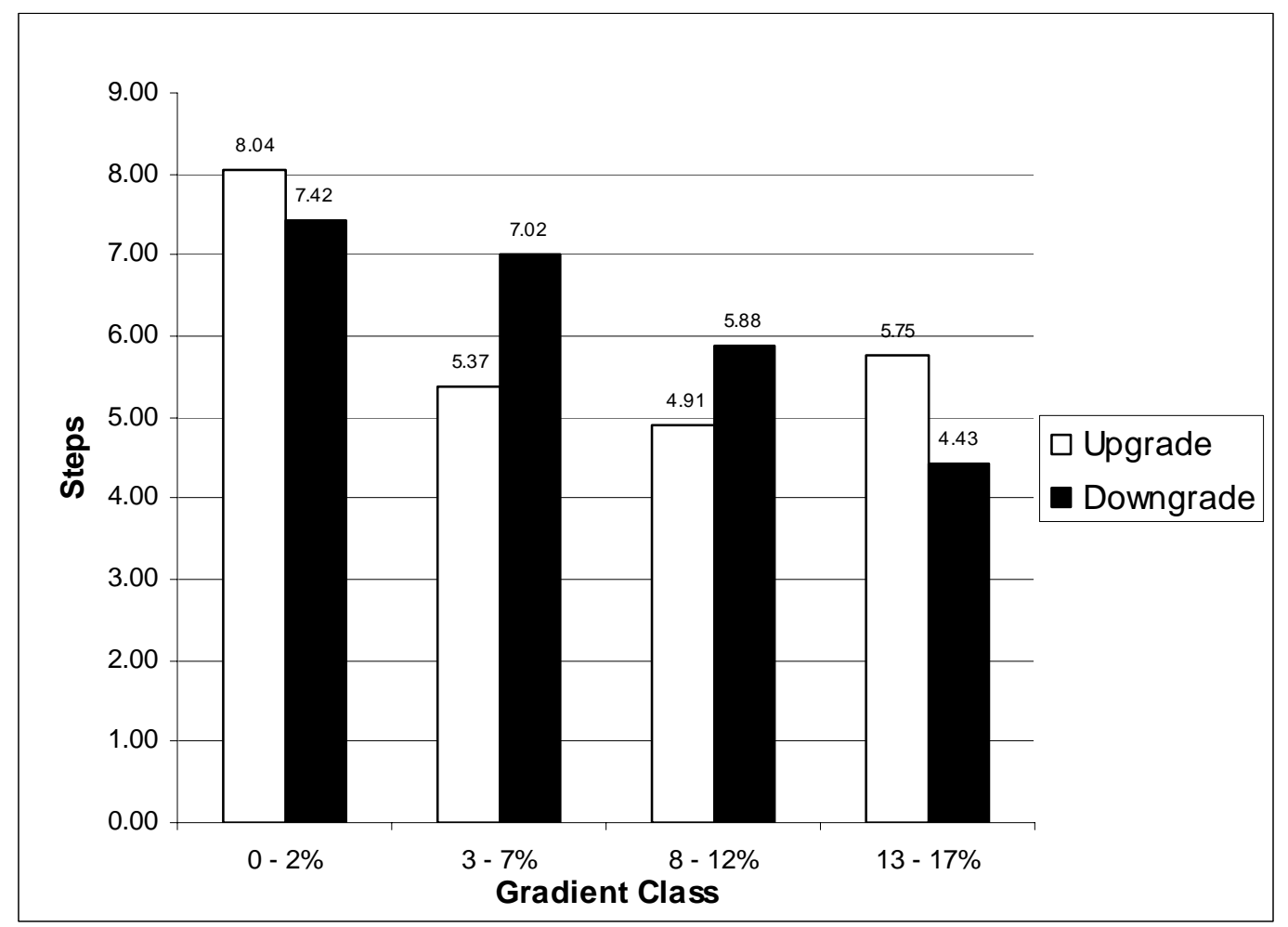

Figure 20: Standard Deviation of Steps for Each Gradient Class

For downgrades, the standard deviation decreased uniformly with increasing gradient. This was the opposite of the outcome expected. It had been anticipated that the lowest variability would occur on the near-level surface and that the variability would 
increase with increasing gradients since subject's walking behavior would be different and stride lengths would change.

There is no obvious explanation for the results. They could be related to the experimental nature of the walking which may have caused participants to focus more on maintaining their stride length than they would naturally (the Hawthorne effect). The results could also be attributed to the fact that the subjects were mainly young adult males. Results could be different if more females and older adults were included in the sample.

T-tests were performed between different gradient classes (assuming equal variance) to determine whether the differences were statistically significant. The hypothesis is shown below,

Null Hypothesis, $\mathrm{H}_{0}$ : Number of Steps on Gradient 1 = Number of Steps on Gradient 2

Alternate Hypothesis, $\mathrm{H}_{1}$ : Number of Steps on Gradient $1 \neq$ Number of Steps on Gradient 2

Results are shown in Table 7. Table 6 is a summary of the P-values obtained from the t-test (95\% confidence level) for different gradients. The P-value is the level of significance for the acceptance or rejection of the hypothesis. Since $95 \%$ confidence has been assumed, any P-value more than 0.05 would lead to acceptance of the null hypothesis.

Table 7 - P-Values Obtained from Comparison of Steps on Different Gradients

\begin{tabular}{|l|r|r|}
\cline { 2 - 3 } \multicolumn{1}{c|}{} & \multicolumn{2}{c|}{$\mathrm{P}(\mathrm{T}<=\mathrm{t})$ two-tail } \\
\cline { 2 - 3 } \multicolumn{1}{c|}{} & Upgrade & Downgrade \\
\hline $0-2 \%$ vs. $3-7 \%$ & 0.790683 & 0.84498775 \\
\hline $3-7 \%$ vs. $8-12 \%$ & 0.666578 & 0.82749289 \\
\hline $8-12 \%$ vs. $13-17 \%$ & 0.820852 & 0.33775823 \\
\hline $0-2 \%$ vs. $8-12 \%$ & 0.951688 & 1 \\
\hline $0-2 \%$ vs. $13-17 \%$ & 0.817476 & 0.41237835 \\
\hline $3-7 \%$ vs. $13-17 \%$ & 0.53911 & 0.53787325 \\
\hline
\end{tabular}


As seen in Table 7, the P-values for each comparison were considerably higher than 0.05 (value below which the null hypothesis would be rejected). In fact, the t-test between the downgrade categories $0-2 \%$ and $8-12 \%$, yielded a P-value of one, indicating absolutely no difference, although this is not theoretically possible. It was inferred from the P-values that the null hypothesis (i.e., Number of steps on Gradient $1=$ Number of steps on Gradient 2) cannot be rejected. Thus, it was concluded that there was no significant difference between numbers of steps required for each of the gradient classes.

There two experiments showed that the pedometer proved to be a reliable device counting the number of steps for the pedestrians regardless of surface type and gradient. The results obtained were in contrast to the outcome expected. There may be various reasons for the obtained results. One of the potential factors may be the Hawthorne effect, also known as the under-observation effect, as described earlier.

Another limitation of the experiment was that the sample size was not representative of the entire population. Most of the subjects who volunteered for the experiment were male graduate students in the age group of 24 to 26 years of age. A more representative sample might yield different results.

\subsubsection{Effect of Social Interaction on Number of Steps}

This experiment was different from the two experiments which assessed the effects of physical characteristics of the walkway on the pedometer accuracy. Even though the experiment involved subjects, they played the role of an outside influence. The data collected were the researcher's step count rather than the subject's step count. 
The experiment was conducted over four days in mid-April, 2005. The site chosen for the experiment was a high-type surface that represented a sidewalk in good condition. The test section was located in front of the Engineering Research Building on the Evansdale Campus of West Virginia University. At the site, a 200-foot long test section was marked beforehand. Five subjects, all male graduate students, volunteered for the experiment. Subjects of varying heights were sought so there would be variation in stride length. All subjects complied with the protocol presented in the previous chapter. Table 8 presents the subject characteristics.

Table 8 - Characteristics of Subjects in the Effect of Social Interaction on Number of Steps Exp
\begin{tabular}{|c|c|c|c|c|}
\hline Subject & Height & Age & Gender & $\begin{array}{c}\text { Stride } \\
\text { Length (ft.) }\end{array}$ \\
\hline 1 & $54^{\prime \prime}$ & 24 & M & 2.15 \\
\hline 2 & $5^{\prime} 8^{\prime \prime}$ & 23 & M & 2.30 \\
\hline 3 & $5^{\prime} 9^{\prime \prime}$ & 23 & M & 2.40 \\
\hline 4 & $5^{\prime} 11^{\prime \prime}$ & 24 & M & 2.40 \\
\hline 5 & $6^{\prime} 1 "$ & 18 & M & 2.55 \\
\hline
\end{tabular}

The average stride length of the researcher when walking alone was calculated as 2.48 feet. The subjects varied in age from 18 to 24 years with a mean of 22.4 years. Subjects varied in height from 5' 4" to 6' 1" with a mean of 5'9". The subjects were taken individually to the test section. Their stride length was measured and recorded on the data sheet shown in Appendix B. Stride length varied from 2.15 to 2.55 feet with a mean of 2.36 feet.

The weather on each of the days of the experiment was similar; tests were carried out during daylight when there was no falling precipitation (partly cloudy with temperatures varying from $60 \mathrm{~F}$ to $72 \mathrm{~F}$ ). All subjects performed the experiment in conjunction with the researcher in the manner described in the procedure. 
The steps shown on the pedometer (Yamax Digiwalker SW651) were recorded on the data sheet (Appendix B) after each subject and the researcher completed each of the 4 trials on the test section. After all test runs were completed, the data were grouped together on a spreadsheet and plotted for analysis. It should be noted that, in order to obtain reproducibility, the test runs were carried out when there was no other pedestrian traffic on the walkway and both the researcher and the accompanying subject had more than enough space to walk. Therefore, the interaction between the researcher and the accompanying subject was done at a greater distance than might be found in actual conversations. Table 9 presents a summary of the number of steps required by the researcher to cover the section under different test conditions. From Table 9, it can be seen that the average number of steps required by the researcher for condition A was 80.6 steps. When the researcher walked with subject 1 (shown in column one), more steps were required to traverse the test section. Subject 1 was the shortest and had the smallest stride length of $2.15 \mathrm{ft}$, compared to other subjects. It appeared that Subject 1's short stride length affected the researcher's stride length.

Table 9 - Number of Steps Required by Researcher for Different Levels of Social Interaction.

\begin{tabular}{|l|c|c|c|c|c|c|c|}
\cline { 2 - 7 } \multicolumn{1}{c|}{} & \multicolumn{9}{c|}{ Steps } & \multicolumn{2}{c|}{} \\
\hline Test Condition & $\begin{array}{c}\text { Subject } \\
\mathbf{1}\end{array}$ & $\begin{array}{c}\text { Subject } \\
\mathbf{2}\end{array}$ & $\begin{array}{c}\text { Subject } \\
\mathbf{3}\end{array}$ & $\begin{array}{c}\text { Subject } \\
\mathbf{4}\end{array}$ & $\begin{array}{c}\text { Subject } \\
\mathbf{5}\end{array}$ & Average & $\begin{array}{c}\text { Std. } \\
\text { Dev. }\end{array}$ \\
\hline $\begin{array}{l}\text { Condition A-Researcher } \\
\text { walking alone }\end{array}$ & 80 & 82 & 82 & 79 & 80 & 80.6 & 1.34 \\
\hline $\begin{array}{l}\text { Condition B-Researcher with } \\
\text { subject -No talking }\end{array}$ & 85 & 81 & 81 & 80 & 80 & 81.4 & 2.07 \\
\hline $\begin{array}{l}\text { Condition C-Researcher with } \\
\text { subject -Limited talking }\end{array}$ & 87 & 83 & 82 & 82 & 81 & 83 & 2.35 \\
\hline $\begin{array}{l}\text { Condition D-Researcher with } \\
\text { subject -Significant conversation }\end{array}$ & 88 & 83 & 83 & 82 & 81 & 83.4 & 2.61 \\
\hline
\end{tabular}

For Condition B, the researcher's step count was 85 walking with Subject 1 versus almost 81 when walking alone. For Conditions $\mathrm{C}$ and $\mathrm{D}$, the step counts with Subject 1 were 87 and 88, respectively. This represents an almost 10\% variation from the 
researcher's average of 80.6 steps. Little difference in the researcher's steps was seen for conditions $\mathrm{B}, \mathrm{C}$ and $\mathrm{D}$.

The researcher, along with subjects 2, 3, 4 and 5 (columns two to five), required almost same number of steps to cover the test section under the different test conditions, even though the stride length was different for each of the subjects. Considering only subjects 2, 3, 4 and 5, there was not much difference in the step count for Condition C; the maximum variation was two steps. Even for Condition D, the variation from Condition A was not more than two steps. Table 8 also shows the column for average number of steps for each condition considering the researcher's steps recorded for each subject. For Condition B, there was almost no difference in the number of steps compared to walking alone. For Conditions $\mathrm{C}$ and $\mathrm{D}$, the variation in step count was only 2 and 3 steps, respectively.

The last column in Table 8 shows the standard deviation for the subjects under different test conditions. The standard deviation increased (although by small values) almost uniformly with an increase in interaction. This shows that interaction between the subject and the researcher increased variability in step count as measured by the pedometer. This result was anticipated.

A t-test between the first condition, i.e. researcher walking alone, and each of the other three conditions (assuming equal variance) was performed to determine whether there were significant differences in number of steps between the levels of social interaction. The hypothesis is shown below. 
Null Hypothesis, $\mathbf{H}_{\mathbf{0}}$ : Number of Steps when Researcher walking alone

$=$ Number of Steps when Researcher walking with subject (No talking, Limited talking, Significant Conversation)

Alternate Hypothesis, $\mathbf{H}_{\mathbf{1}}$ : Number of Steps when Researcher walking alone

$\neq$ Number of Steps when Researcher walking with subject (No talking, Limited talking, Significant Conversation)

Table 10 presents a summary of the P-values obtained from the t-test $(95 \%$ confidence level) for different test-conditions. The P-value is the level of significance for the acceptance or rejection of the hypothesis. Since $95 \%$ confidence has been assumed, any P-value more than 0.05 would lead to acceptance of the null hypothesis.

Table 10 - P-Values Obtained from Comparison of Steps for Different Levels of Social Interaction.

\begin{tabular}{|l|c|}
\hline \multicolumn{1}{|c|}{ Comparison } & P(T<=t) two-tail \\
\hline Researcher walking alone Vs. Researcher with subject (No talking) & 0.489527061 \\
\hline Researcher walking alone Vs. Researcher with subject (Limited talking) & 0.08224863 \\
\hline Researcher walking alone Vs. Researcher with subject (Significant conversation) & 0.071614557 \\
\hline
\end{tabular}

From Table 10, it can be seen that P-values for all comparisons are more than 0.05, thus leading to failure to reject the Null Hypothesis $\mathrm{H}_{0}$. Note, however, that the Pvalues for the second and third comparisons are quite close to the value of 0.05 . The results indicated social interaction has no effect on the number of steps. This finding is at odds with what would be expected intuitively, i.e., social interaction between individuals while walking would affect stride length and, therefore, number of steps.

The obtained result may be due to a number of factors. The experimental method involved conversations (both limited and significant) which were at least partially scripted beforehand to make the experiment reproducible. This artificial or contrived conversation may not have adequately reflected the spontaneous nature of actual social interaction. Also, as mentioned before, the interaction between the researcher and the 
accompanying subject was done at a greater distance than might be found in actual conversations. This may have been partially due to the fact that the test section was significantly wider than the typical sidewalk so it was easier to increase one's personal space.

All individuals involved were aware that an experiment was being carried out and, thus, subconsciously there may have been a change in their walking characteristics, which they knew were being studied. This is the Hawthorne effect described earlier. Additional research to address the elements of social interaction is recommended. The pedometer proved to be an easy to use device and displayed steps correctly, but some improvements such as a data storage capacity are needed in order to make the experiments more efficient.

\subsubsection{Effect of Other Pedestrian Traffic on Number of Steps}

This experiment was different from all of the previous experiments assessing pedometer accuracy. The experiment did not involve any subjects and the data collected were the researcher's step count, under different pedestrian traffic conditions on a walkway. The varying pedestrian traffic played the role of the outside influence and no experimental setup was prepared beforehand. The experiments were conducted in midto late-April.

Once the test runs were conducted, step counts recorded and photographs of runs printed, the level of service at which the facility operated during each run was determined for all runs (as described in Chapter 3). The number of steps recorded by the researcher to cover the length of the test section for each LOS was compared and analyzed. 
The original goal was to obtain 5 test runs for each LOS, from A to C. This goal was partially achieved as, after the photos were analyzed, it was determined that only one test run was obtained for LOS C. Additional runs were made in an effort to obtain data for LOS C; however they proved unsuccessful. The main reason for only one run falling under LOS C category was insufficient pedestrian traffic on the test sections.

The runs were carried out at two locations as mentioned in previous chapter. The test section on Evansdale Campus was 1200 sq. $\mathrm{ft}$. in area. The test section on the Downtown Campus was 500 sq. $\mathrm{ft}$. in area. Table 1 in Chapter 3 presented the values for Pedestrian Space Requirements for each level of service as given in the Highway Capacity Manual (TRB, 2000). Table 11 presents a summary of the number of steps the researcher took to cover the test section for each of the test runs at different levels of service.

Table 11 - Data Obtained After Analysis of All Test Runs for Effect of Other Pedestrian Traffic on Number of Steps

\begin{tabular}{|c|c|c|c|c|c|c|}
\hline \multicolumn{2}{|c|}{ Test Condition } & $\begin{array}{c}\text { No. of } \\
\text { Pedestrians } \\
\end{array}$ & $\begin{array}{l}\text { Area of } \\
\text { Section } \\
\text { (sq.ft) }\end{array}$ & $\begin{array}{c}\text { Pedestrian } \\
\text { Space } \\
\text { (sq.ft./ped.) }\end{array}$ & $\begin{array}{c}\text { Number of } \\
\text { Steps }\end{array}$ & $\begin{array}{c}\text { Average Steps } \\
\text { for } \\
\text { Each LOS } \\
\end{array}$ \\
\hline \multicolumn{2}{|c|}{ Researcher Alone } & & & & 41 & 41 \\
\hline & Run 1 & 12 & 1200 & 100.00 & 41 & \multirow{5}{*}{42.4} \\
\hline & Run 2 & 15 & 1200 & 80.00 & 43 & \\
\hline \multirow[t]{5}{*}{ LOS A } & Run 3 & 17 & 1200 & 70.59 & 43 & \\
\hline & Run 4 & 8 & 500 & 62.50 & 43 & \\
\hline & Run 5 & 8 & 500 & 62.50 & 42 & \\
\hline & Run 1 & 9 & 500 & 55.56 & 41 & \multirow{5}{*}{42.8} \\
\hline & Run 2 & 10 & 500 & 50.00 & 43 & \\
\hline \multirow[t]{3}{*}{ LOS B } & Run 3 & 10 & 500 & 50.00 & 43 & \\
\hline & Run 4 & 12 & 500 & 41.67 & 44 & \\
\hline & Run 5 & 12 & 500 & 41.67 & 43 & \\
\hline LOS C & Run 1 & 13 & 500 & 38.46 & 44 & 44 \\
\hline
\end{tabular}

From Table 11, it can be seen that the average number steps for levels of service A and B vary only slightly from the number of steps required by the researcher when 
walking alone with no other pedestrian traffic present. Even for LOS C, the single run required only 44 steps which are not much different from the number of steps recorded for levels of service A and B. The difference is less than 10 percent. Of course, additional runs would be required at LOS C to draw any sort of conclusions.

It was expected that more steps would be taken as pedestrian density increased (i.e., pedestrian space decreased) but obviously the data were not sufficient to confirm this. There maybe a few factors responsible for this hypothesis. The space per pedestrian is directly related to the area of test section and inversely related to the number of pedestrians. The area of test section 1 gave pedestrians ample space to maneuver and pass other pedestrian traffic, even though it had a maximum number of 17 pedestrians. In retrospect, test section 1 was not well suited for this experiment since it handled relatively small volumes of pedestrians on its relatively wide width. The situation would have been totally different if the same traffic was present on a 6 feet wide sidewalk. On test section 2, the width, although less, had the pedestrian traffic scattered throughout the section and thus did not affect the researcher's stride as much. On the whole, the level of service is primarily function of pedestrian volumes. Volumes on the WVU campus were just too low for the purposes of the study. Thus, an urban location having significantly higher pedestrian volumes on sidewalks is recommended for this experiment.

From this experiment, it was observed that though the pedometer was easy to use and performed well for counting steps, it was felt that the pedometer required a data storage capacity since the step count from each of the run was required to be recorded on a separate data sheet (Appendix B), which sometimes proved to be a drawback. 


\section{Summary of Pedometer Evaluation}

Results of the pedometer experiment showed that the different surfaces and different gradients had no significant effect on stride length and, therefore, step count. This result differed from what had been intuitively expected. Social interaction had no significant effect on pedometer step count in this study. In the researcher's opinion, this was due to the inability of the experimental setup to replicate real-world situations. The data for 'Effect of Other Pedestrian Traffic' showed that under LOS A and LOS B, the stride length did not change significantly, due to the low density of pedestrian traffic. There was some indication of a change at LOS C but the data were not sufficient to draw any conclusions. The Hawthorne Effect could explain the results obtained. It must also be noted that the subjects were rather homogenous (primarily college-age males) and thus not representative of the population at large. A larger, more representative, sample with varying ages and gender is required to get more reliable result in all of these experiments. The combined effect (interactions) of the experimental conditions, such as, effect of gradient along with other pedestrian traffic or effect of surface along with social interaction between individuals, on the step count, was not examined. Additional research is recommended in order to better understand the effects of above-mentioned factors on step count.

On this basis, it is concluded that, the pedometer provides an accurate measure of step count, and in turn, distance traveled by pedestrians. However there are questions about its data storage capabilities and operational considerations. These will be assessed later in this chapter. 


\subsection{Evaluation of Device Characteristics - Hand-Held GPS Unit and Wearable \\ GPS Data-Logger}

The following sections discuss the characteristics of the two GPS devices in the context of collecting data under different experimental conditions. The data collected by the two devices for each experiment are presented in the form of tables. There are two types of tables obtained from the two devices, i.e., those for the hand-held GPS unit and those produced by the wearable GPS data-logger. The tables are de-coded below in order to understand the various column headings and the abbreviations used.

Tables 12 and 13 present descriptions of the various fields in the table obtained from the hand-held GPS unit and the wearable GPS data-logger, respectively.

Table 12 - Description of Various Fields in the Tabular Data Obtained from the Hand-Held GP
\begin{tabular}{|c|c|}
\hline Field 1 & $\begin{array}{c}\text { Field Information } \\
\text { T - track point }\end{array}$ \\
\hline Field 2 & Latitude N (S) (ddmmss.ss) \\
\hline Field 3 & Longitude E (W) (dddmmss.ss) \\
\hline Field 4 & Altitude (meters) \\
\hline Field 5 & Date (mm/dd/yyyy) \\
\hline Field 6 & Time GMT (Greenwich Mean Time) (hhmmss) \\
\hline Field 7 & Start Code '1' = new segment start, '0' = same segment \\
\hline Field 8 & Elapsed time (seconds) \\
\hline Field 9 & Distance from start (meters) \\
\hline Field 10 & Speed (m/s) \\
\hline
\end{tabular}

Through analysis of data recorded in the tabular forms shown, the time and duration of the loss of satellite signal can be determined. In the case of a hand-held GPS unit, a gap in the otherwise continuous data denotes a loss of signal. Also, a ' 1 ' in field 7 for the start code, indicates a loss of signal. The ' 1 ' denotes a new segment which started as result of loss of signal or when the device is turned on. 
Table 13 - Description of Various Fields in the Tabular Data Obtained from the Wearable GPS Data-Logger

\begin{tabular}{|c|c|}
\hline Field 1 & Record Count (Integer) \\
\hline Field 2 & $\begin{array}{c}\text { A = valid data, GPS ok; D = valid data, DGPS ok; V } \\
\text { first valid sentence after loss of signal or power }\end{array}$ \\
\hline Field 3 & Date (mm/dd/yy) \\
\hline Field 4 & Local Time (hh:mm:ss) \\
\hline Field 5 & Latitude (ddmm.mmm) OR (dd.ddddd) \\
\hline Field 6 & Longitude (dddmm.mmm) OR (dd.ddddd) \\
\hline Field 7 & Speed-mph OR kph (000.0 - 114.9) \\
\hline Field 8 & Heading-degrees (000 - 259) \\
\hline Field 9 & Altitude (ff.f) OR (mm.m) \\
\hline Field 10 & Horizontal Dilution Of Precision (HDOP) (00.5 - 99.9) \\
\hline Field 11 & Satellites (00 - 12) \\
\hline
\end{tabular}

Recorded data from one of the experiments is presented as an example in Table

14.

Table 14 -Recorded Data from One Experiment Using the Hand-Held GPS Device

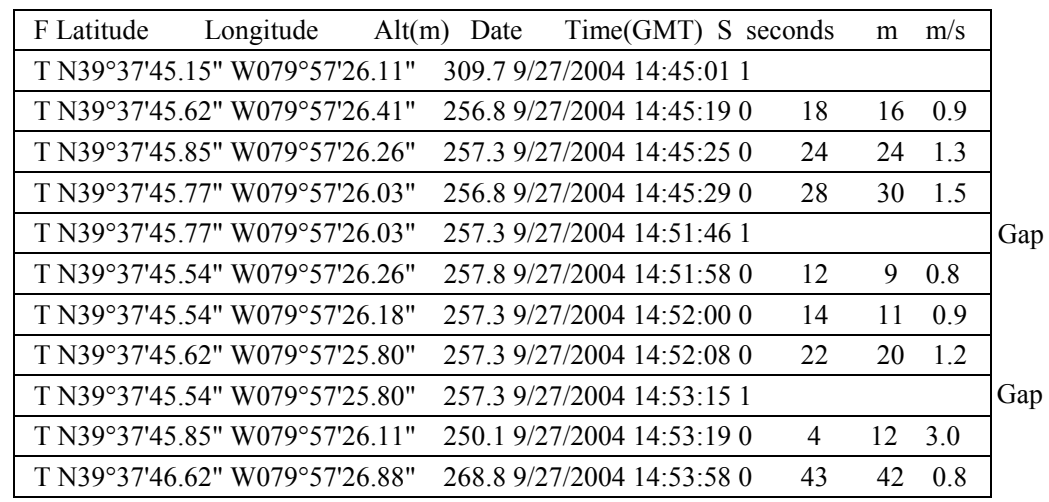

From the above table two gaps in the continuous rows of data can be observed.

These gaps represent loss of signal at two different instances during the shown trip. The loss of signal can be identified looking at the ' 1 ' in field 7 for the start code. The ' 1 ' denotes a new segment which started as result of loss of signal or when the device is turned on.

For the wearable GPS data-logger, the loss of signal can be determined by looking at the field ' 1 ' for flag. $A$ ' $V$ ' in this field indicates the first valid sentence after loss of signal or power. Signal loss can also be located in the data by looking at fields 7 , field 8 or field 11. A zero in these fields indicates a loss of signal. Recorded data from one of the experiments is presented as an example in Table 15. The rows in the table have been 
shaded only for reference and represent a few instances of signal loss. As can be seen, a ' 0 ' is present in fields 7,8 and 11 , indicating a loss of signal.

Table 15 -Recorded Data from One Experiment Using the Wearable GPS Data-Logger

\begin{tabular}{|l|}
\hline RECORD,FLAG,DATE,TIME,LAT,LONG,SPEED,HEAD,ALTITUDE,HDOP,SATS \\
\hline $269, \mathrm{~A}, 03 / 19 / 05,11: 29: 48,40.44100,-80.0038,002.7,292,00764,03.6,03$ \\
\hline $270, \mathrm{~V}, 03 / 19 / 05,11: 29: 53,40.44105,-80.00387,002.8,290,00761,03.0,04$ \\
\hline $271, \mathrm{~A}, 03 / 19 / 05,11: 29: 58,40.44112,-80.00392,000.0,000,00754,04.6,04$ \\
\hline $272, \mathrm{~V}, 03 / 19 / 05,11: 30: 03,40.44113,-80.004,000.0,000,00000,00.0,00$ \\
\hline $273, \mathrm{~V}, 03 / 19 / 05,11: 30: 11,40.44033,-80.00622,000.0,000,00771,02.9,04$ \\
\hline $274, \mathrm{~V}, 03 / 19 / 05,11: 30: 34,40.45815,-80.01258,000.0,000,00771,28.2,00$ \\
\hline $275, \mathrm{~V}, 03 / 19 / 05,11: 30: 38,40.44062,-80.00347,000.0,000,00797,02.2,00$ \\
\hline $276, \mathrm{~V}, 03 / 19 / 05,11: 30: 46,40.44000,-80.00642,000.0,000,00000,00.0,00$ \\
\hline $277, \mathrm{~V}, 03 / 19 / 05,11: 30: 51,40.43923,-80.00353,000.0,000,00820,05.2,00$ \\
\hline $278, \mathrm{~V}, 03 / 19 / 05,11: 30: 54,40.43943,-80.00632,000.0,000,00817,03.0,00$ \\
\hline $279, \mathrm{~V}, 03 / 19 / 05,11: 30: 59,40.43810,-80.00332,000.0,000,00830,05.3,00$ \\
\hline $280, \mathrm{~V}, 03 / 19 / 05,11: 31: 03,40.44042,-80.00413,000.0,291,00830,02.8,00$ \\
\hline $281, \mathrm{~A}, 03 / 19 / 05,11: 31: 08,40.44058,-80.00467,002.0,181,00899,05.3,05$ \\
\hline
\end{tabular}

\subsubsection{Signal Availability Under High-Voltage Power Lines and Transmission Towers}

The two units, i.e., the hand-held GPS unit and the wearable GPS data-logger, were switched on as the researcher walked near the power lines. The hand-held GPS unit took about two minutes to acquire a good signal from the satellites and to configure itself. The GPS devices required a signal from a minimum of 4 satellites in order to correctly give the position of the user. The 'minimum 4 satellite signal' criteria are necessary to get the longitude, latitude, altitude and time for each of the records. The display screen of the unit, showed a strong signal from 5 satellites (and a weak signal from 2 more) and the accuracy was $16 \mathrm{ft}$. There was almost no change in the accuracy shown on the handheld GPS unit as the researcher walked towards a spot under the power lines. Even after the researcher stood directly under the high-voltage power lines for four to five minutes, there was no significant change in the accuracy displayed by the GPS unit. The display on the hand-held GPS still showed a strong signal from 5 satellites and the accuracy value fluctuated between 15 and 17 feet. 
The researcher then walked towards one of the transmission line towers to which the high-voltage power lines were connected. As shown in Figure 12, the tower was a steel truss-like structure. The point reached was about 20 feet away from the tower. There was no evidence of loss of signal or change in accuracy and the hand-held GPS again displayed a strong signal from 6 satellites and an accuracy value around 16 feet. There was no evidence that the steel transmission tower affected the signal received by the hand-held GPS unit. This process was repeated for a different transmission line on another tower located some distance away. The hand-held GPS unit again displayed a strong signal from 6 satellites with the displayed accuracy value of 15-16 feet. There was no evidence of loss of signal or change in accuracy as a result of the overhead highvoltage power lines

After returning from the field, the GPS units were connected to a computer to download the data for analysis. The data retrieved from both GPS units showed no loss of signal (based on the criteria described in Chapter 3) during the time period when the experiment was conducted. The wearable GPS data logger unit was a more powerful unit (in terms of sensitivity of the receiver antenna), used specifically for transportation data collection, thus it was able to connect to more satellites with its receiver. The data from the wearable GPS data logger unit showing number of satellite connections was almost constant at 9. Also, there was no gap found in the active log of the hand-held GPS unit data, which meant that data were received continuously. This procedure just described was performed for two different locations, near the coal-fired power plant, on the same date. The two locations were about 2 miles apart. 
A sample of the data collected is shown in Table 16. As can be observed from Table 16, the hand-held unit was receiving data continuously during the time period of the experiment.

Table 16 - Sample Data Obtained from Hand-Held GPS Unit for Effect of High-Voltage Power Lines and Transmission Towers on Availability of Satellite Signal

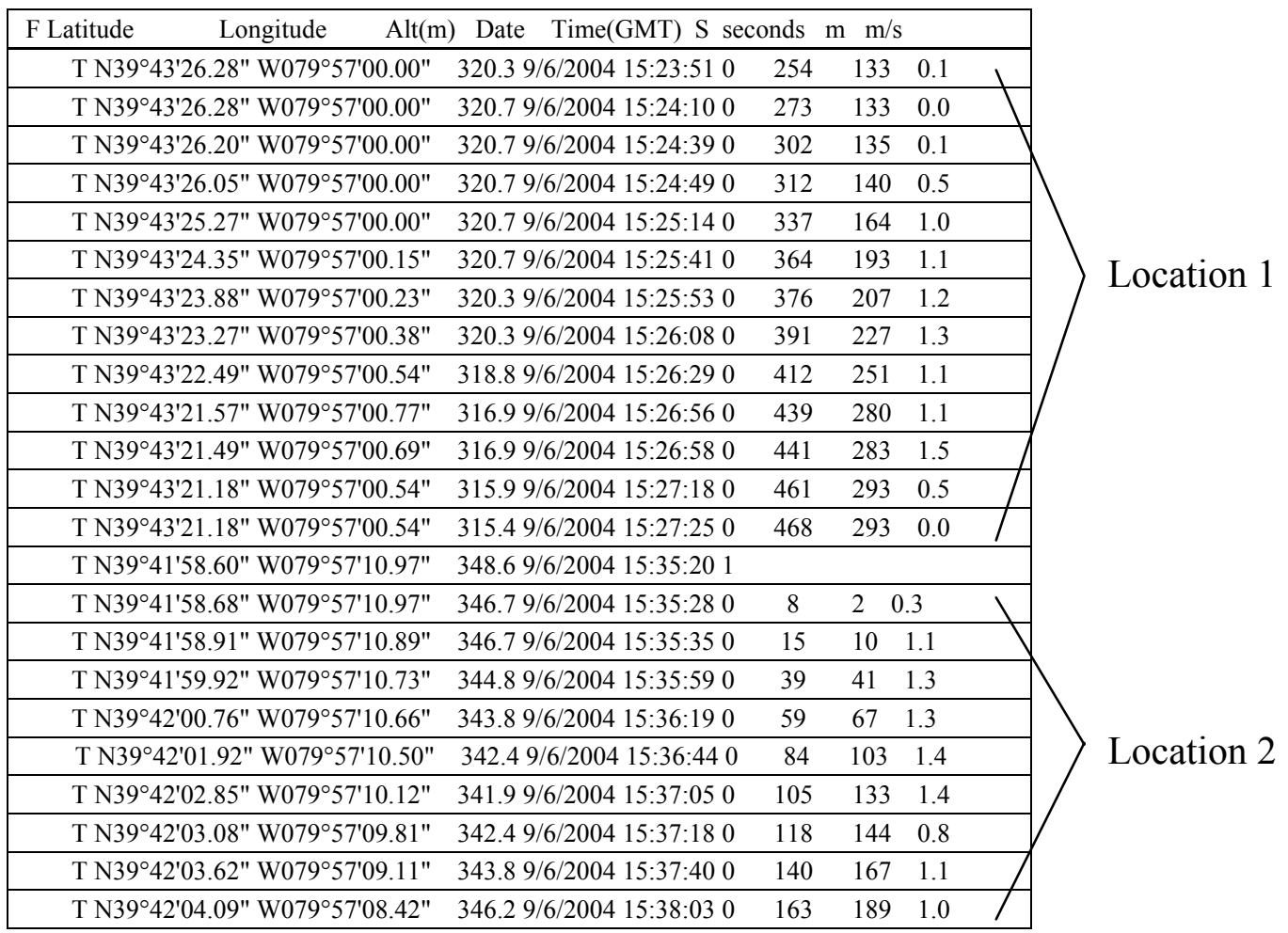

Table 17 presents a sample of the data collected from the wearable data logger.

From the table, it can be seen that the GPS device recorded data at regular intervals of 5 seconds. The last column of the table shows that the number of satellites to which the device was linked was constant at 9 and that there was no loss of signal during the entire duration of the experiment.

After recording observations of number of satellites from the hand-held GPS unit and analyzing data downloaded and recorded from the display screen for the two GPS units, it was concluded that there was no loss of signal availability under high-voltage power lines and in the vicinity of transmission towers under the stated conditions. 
Table 17 - Sample Data Obtained from Wearable GPS Data-Logger Unit for Effect of High-Voltage Power Lines and Transmission Towers on Availability of Satellite Signal

\begin{tabular}{|l|}
\hline RECORD,FLAG,DATE,TIME,LAT,LONG,SPEED,HEAD,ALTITUDE,HDOP,SATS \\
\hline $308, \mathrm{~A}, 09 / 06 / 04,11: 24: 56,39.72385,-79.95,1.9,180,1063,0.9,9$ \\
\hline $309, \mathrm{~A}, 09 / 06 / 04,11: 25: 01,39.7238,-79.94998,2,178,1063,0.9,9$ \\
\hline $310, \mathrm{~A}, 09 / 06 / 04,11: 25: 06,39.72377,-79.94998,2,179,1063,0.9,9$ \\
\hline $311, \mathrm{~A}, 09 / 06 / 04,11: 25: 11,39.72372,-79.95,2.2,181,1063,0.9,9$ \\
\hline $312, \mathrm{~A}, 09 / 06 / 04,11: 25: 16,39.72367,-79.95,2.2,181,1063,0.9,9$ \\
\hline $313, \mathrm{~A}, 09 / 06 / 04,11: 25: 21,39.72362,-79.95,2.4,182,1063,0.9,9$ \\
\hline $314, \mathrm{~A}, 09 / 06 / 04,11: 25: 26,39.72358,-79.95,2.8,187,1063,0.9,9$ \\
\hline $315, \mathrm{~A}, 09 / 06 / 04,11: 25: 31,39.72355,-79.95,2.2,185,1063,1.2,9$ \\
\hline $316, \mathrm{~A}, 09 / 06 / 04,11: 25: 36,39.7235,-79.95,2.5,185,1059,1,9$ \\
\hline $317, \mathrm{~A}, 09 / 06 / 04,11: 25: 41,39.72345,-79.95002,2.5,185,1059,0.9,9$ \\
\hline $318, \mathrm{~A}, 09 / 06 / 04,11: 25: 46,39.72338,-79.95002,2.5,183,1059,1,9$ \\
\hline $319, \mathrm{~A}, 09 / 06 / 04,11: 25: 51,39.72335,-79.95003,2.4,187,1059,1.3,9$ \\
\hline $320, \mathrm{~A}, 09 / 06 / 04,11: 25: 56,39.72328,-79.95003,2.6,189,1059,0.9,9$ \\
\hline $321, \mathrm{~A}, 09 / 06 / 04,11: 26: 01,39.72323,-79.95005,2.7,189,1056,0.9,9$ \\
\hline $322, \mathrm{~A}, 09 / 06 / 04,11: 26: 06,39.72317,-79.95007,2.7,189,1056,0.9,9$ \\
\hline $323, \mathrm{~A}, 09 / 06 / 04,11: 26: 11,39.72312,-79.95007,2.6,190,1056,0.9,9$ \\
\hline $324, \mathrm{~A}, 09 / 06 / 04,11: 26: 16,39.72307,-79.95008,2.5,191,1056,0.9,9$ \\
\hline $325, \mathrm{~A}, 09 / 06 / 04,11: 26: 21,39.72302,-79.9501,2.5,190,1056,0.9,9$ \\
\hline
\end{tabular}

Before drawing general conclusion, additional research is recommended given the limited conditions examined. In this experiment, only one type of high-voltage power line was considered, the effect may vary with the different types or configurations of power lines. Also the effect of other conditions, for example, rain, in combination with proximity to power lines was not examined. In this case, the distance between the power line and the GPS devices remained essentially constant and thus the effect of distance was not considered.

\subsubsection{Signal Availability Under Dense Tree Canopy}

The two units were switched on as the researcher walked towards the tree canopy. The hand-held GPS unit took about two minutes to acquire a good signal from the satellites and to configure itself. The unit showed a strong signal from 4 satellites (and a weak signal from 1 more) and the accuracy was 18 feet. Upon entering the tree canopy, there was a strong signal from 5 satellites and the accuracy shown on the hand-held GPS unit changed to 16 feet. Even after the researcher walked under the dense tree cover for 
four to five minutes, there was no significant change in the accuracy shown by the handheld GPS unit. The display on the hand-held GPS still showed a strong signal from 5 satellites and the accuracy fluctuated between 15 and 17 feet.

Table 18 presents the data received from the hand-held GPS unit after it was connected to a computer. It can be seen in Table 18 that there are two gaps (as indicated) in an otherwise continuous active log recorded by the hand-held GPS unit. These gaps indicate a total loss of signal from the satellites. The first loss of signal was for duration of approximately 59 seconds, whereas the second loss lasted approximately 51 seconds. This can be determined by taking out the time difference between the record where the gap is found and the previous record (e.g. 18:12:51 - 18:11:52 $=59$ seconds).

Table 18 - Sample Data Obtained from Hand-held GPS Unit for Effect of Dense Tree Canopy on Availability of Satellite Signal

\begin{tabular}{|c|c|c|c|c|}
\hline Longitude & Alt(m) Date & secon & $\mathrm{s} \mathrm{m}$ & $\mathrm{m} / \mathrm{s}$ \\
\hline T N39³8'50.04" W07958'42.43" & " $317.49 / 5 / 2004$ 18:08:13 0 & 156 & 112 & 0.9 \\
\hline T N39॰38'49.42" W07958'43.59" & 314.5 9/5/2004 18:08:48 0 & 191 & 145 & 1.0 \\
\hline T N39॰38'48.65" W07958'44.28" & $313.09 / 5 / 200418: 09: 170$ & 220 & 174 & 1.0 \\
\hline T N39॰38'48.65" W07958'44.36" & $313.09 / 5 / 2004$ 18:09:18 0 & 221 & 176 & 1.8 \\
\hline T N39³8'48.42" W07958'44.98" & $311.19 / 5 / 2004$ 18:09:40 0 & 243 & 193 & 0.7 \\
\hline T N39॰38'48.03" W07958'45.75" & $310.29 / 5 / 200418: 10: 310$ & 294 & 214 & 0.4 \\
\hline T N39॰38'47.26" W07958'46.44" & 307.8 9/5/2004 18:11:04 0 & 327 & 243 & 0.9 \\
\hline T N39॰38'46.64" W07958'46.98" & $308.29 / 5 / 200418: 11: 520$ & 375 & 266 & 0.5 \\
\hline T N39³8'47.80" W07958'46.13" & $285.69 / 5 / 200418: 12: 511$ & & & \\
\hline T N39॰38'47.72" W07958'45.90" & $299.19 / 5 / 2004$ 18:13:07 0 & 16 & 6 & 0.4 \\
\hline T N39³8'48.11" W07958'45.75" & " $302.59 / 5 / 2004$ 18:13:24 0 & 33 & 18 & 0.7 \\
\hline T N39³8'49.04" W07958'44.82" & $302.59 / 5 / 200418: 13: 560$ & 65 & 55 & 1.1 \\
\hline T N39॰38'49.27" W07958'44.13" & $302.59 / 5 / 200418: 14: 290$ & 98 & 73 & 0.5 \\
\hline T N39³8'49.35" W07958'44.20" & $302.09 / 5 / 200418: 14: 520$ & 121 & 76 & 0.1 \\
\hline T N39³8'50.19" W07958'40.34" & $303.49 / 5 / 200418: 15: 431$ & & & \\
\hline T N39³8'49.89" W07958'41.27" & $328.49 / 5 / 200418: 15: 580$ & 15 & 24 & 1.6 \\
\hline T N39³8'49.27" W07958'40.34" & " $323.69 / 5 / 200418: 16: 270$ & 44 & 53 & 1.0 \\
\hline $\begin{array}{lll}\text { F Latitude } & \text { Longitude }\end{array}$ & Alt(m) Date Time(GMT) S & secon & $\mathrm{s} \mathrm{m}$ & $\mathrm{m} / \mathrm{s}$ \\
\hline T N39³8'48.65" W07958'40.26" & " $326.09 / 5 / 200418: 16: 510$ & 68 & 72 & 0.8 \\
\hline T N39³8'48.26" W07958'40.19" & $325.59 / 5 / 2004$ 18:17:010 & 78 & 84 & 1.2 \\
\hline T N39³8'48.03" W07958'40.26" & $324.19 / 5 / 2004$ 18:17:190 & 96 & 92 & 0.4 \\
\hline T N39³8'48.03" W07958'40.26" & $321.79 / 5 / 200418: 18: 150$ & 152 & 92 & 0.0 \\
\hline T N39³8'47.49" W07958'40.50" & 319.8 9/5/2004 18:18:38 0 & 175 & 109 & 0.8 \\
\hline T N39³8'46.64" W07958'41.34" & $318.39 / 5 / 2004$ 18:19:150 & 212 & 143 & 0.9 \\
\hline T N39³8'46.10" W07958'42.27" & $318.39 / 5 / 200418: 19: 560$ & 253 & 170 & 0.7 \\
\hline
\end{tabular}

These signal losses may be considered as a problem in data collection as each of the losses accounts for about 3 records that could have been made if there was signal. 
The ' 3 records' is an estimate of the number of records made by the GPS device in approximately 1 minute. User location will not be recorded during the period of signal loss.

It is important to note that, for data recorded, the display screen in the field did not indicate a loss of signal. This shows that while the hand-held GPS unit may lose signal under thick tree cover, this can only be determined by looking at the downloaded data. Signal loss is not detectable by merely looking at the display screen and observing changes in the satellite signals shown.

The sample of data downloaded from the wearable GPS data-logger is shown in Table 19. From Table 19, it can be observed that data was recorded at 5-second intervals. The second column (flag) indicates signal availability. A ' $\mathrm{V}$ ' indicates first valid sentence after loss of signal or power.

Table 19 - Sample Data Obtained from Wearable GPS Data-Logger Unit for Effect of Dense Tree Canopy on Availability of Satellite Signal

\begin{tabular}{|l|}
\hline RECORD,FLAG,DATE,TIME,LAT,LONG,SPEED,HEAD,ALTITUDE,HDOP,SATS \\
\hline 63,A,09/05/04,14:10:19,39.64678,-79.97922,0,224,1020,1.7,6 \\
\hline $64, \mathrm{~A}, 09 / 05 / 04,14: 10: 24,39.64675,-79.97927,1.4,224,1027,2.1,6$ \\
\hline $65, \mathrm{~A}, 09 / 05 / 04,14: 10: 29,39.6467,-79.9793,1.9,219,1023,1.9,6$ \\
\hline $66, \mathrm{~A}, 09 / 05 / 04,14: 10: 34,39.64667,-79.97933,2,221,1007,1.5,5$ \\
\hline $67, \mathrm{~A}, 09 / 05 / 04,14: 10: 39,39.64663,-79.97937,2,224,1007,1.8,6$ \\
\hline $68, \mathrm{~A}, 09 / 05 / 04,14: 10: 44,39.6466,-79.9794,2,218,1004,2.1,6$ \\
\hline $69, \mathrm{~A}, 09 / 05 / 04,14: 10: 49,39.64657,-79.97943,1.9,225,994,1.8,5$ \\
\hline $70, \mathrm{~V}, 09 / 05 / 04,14: 10: 54,39.64655,-79.97947,1.9,223,997,2.2,6$ \\
\hline $71, \mathrm{~V}, 09 / 05 / 04,14: 10: 59,39.64652,-79.9795,0,0,1000,6.8,0$ \\
\hline $72, \mathrm{~A}, 09 / 05 / 04,14: 11: 04,39.6465,-79.97952,1.5,220,1004,3.6,5$ \\
\hline $73, \mathrm{~A}, 09 / 05 / 04,14: 11: 09,39.64647,-79.97953,1.4,209,1004,1.9,6$ \\
\hline $74, \mathrm{~A}, 09 / 05 / 04,14: 11: 14,39.64647,-79.97952,0,209,994,1.9,6$ \\
\hline $75, \mathrm{~A}, 09 / 05 / 04,14: 11: 19,39.64645,-79.97952,0,0,984,1.9,6$ \\
\hline $76, \mathrm{~A}, 09 / 05 / 04,14: 11: 24,39.64645,-79.97952,0,209,987,2.3,6$ \\
\hline $77, \mathrm{~A}, 09 / 05 / 04,14: 11: 29,39.64643,-79.9795,0,209,977,3.1,6$ \\
\hline $78, \mathrm{~A}, 09 / 05 / 04,14: 11: 34,39.64643,-79.9795,0,209,974,1.7,6$ \\
\hline $79, \mathrm{~A}, 09 / 05 / 04,14: 11: 39,39.64642,-79.97947,1,209,971,2.4,6$ \\
\hline $80, \mathrm{~A}, 09 / 05 / 04,14: 11: 44,39.64638,-79.97943,0,0,974,9.9,6$ \\
\hline
\end{tabular}

In the above sample, for record number 70 and 71 , a ' $\mathrm{V}$ ' under the 'Flag' column, indicates that there was a loss of signal after previous entry, i.e., record number 69 , for 
duration of less than 5 seconds. This duration of loss of signal is insignificant as all records in the downloaded data were made after an interval of 5 seconds. In the entire data set downloaded, a few more instances of loss of signal were recorded but none of them exceeded duration of 5 seconds. Other than these few instances, the wearable GPS data-logger received a good signal from 6 to 8 satellites throughout the period, which can be noted from the last column of the sample data.

The second location for determining the effect of thick tree canopy on signal availability was near the Creative Arts Center on the Evansdale Campus of West Virginia University. This location had a cluster of coniferous trees close to each other. The two GPS units were switched on as the researcher neared the location. After the hand-held GPS unit showed a good signal on the display screen, any changes in the number of satellites or the accuracy at that instance were recorded. After about 2 minutes of startup, the GPS unit showed a strong signal from 5 satellites and accuracy varied between 17 and 18 feet. The researcher stood between and almost under the coniferous trees for 4 to 5 minutes and observed any changes in the satellite signal displayed on the screen. The only changes observed were in the accuracy which improved to 16 feet and the display screen showed a strong signal from 5 to 6 satellites for the entire duration. After downloading the data, no gaps in the otherwise continuous active log were observed, suggesting that thick foliage of coniferous trees did not affect signal availability for the hand-held GPS unit.

The data obtained from the wearable GPS data-logger showed one instance of loss of signal for 11 seconds and two more instances where loss of signal was observed for 
less than 5 seconds. No other instances of signal loss were observed and a good satellite signal (no loss of records) was observed throughout.

From the analysis of the data, it was determined that the wearable GPS unit was better able to maintain a strong satellite signal in comparison to the hand-held GPS unit, which lost signal midway at couple of instances, for a considerable time (durations of almost a minute).

For the purpose of collecting pedestrian data, it can be said that both devices acquired the satellite signal easily under thick vegetation. The hand-held GPS unit lost signal in a few instance and thus may be affected under thick tree cover. Therefore, some points along a route may not be recorded. Thus, there are situations in terms of the types of vegetative cover which present limitations for collecting pedestrian data. Since the experiment was performed for only two types of tree cover, further research would be desirable to study the effect under different types of tree cover, such as under a dense forest or particularly dense species. Further additional work is also recommended in order to ascertain the interaction effects of other factors such as topography and precipitation in combination with dense tree cover.

\subsubsection{Signal Availability in Heavy Rain}

The precipitation for this day of experiment was found to be 1.4 inches as the remnants of a tropical storm passed through the Morgantown area. The intensity of rain was approximately constant during the entire duration of the experiment. However, data were not available on the rainfall intensity (in inches per hour) during the actual conduct of the study. 
The two GPS units were switched on as the researcher came out of the Mineral Resources Building at West Virginia University. After waiting for two minutes, the hand-held GPS showed a strong signal from 4 satellites and a weak one from 2 more with an accuracy of 20 feet. At this point, the researcher started walking towards the Coliseum. The screen display changed to 6 strong signals and accuracy increased to 15 feet after about 3 minutes. The accuracy varied from 15 to 16 feet but the satellite signals remained constant at 6 strong ones. The total duration of the experiment was 5 to 6 minutes.

Table 20 shows the data obtained from the hand-held GPS. As can be noted from the table, there are no gaps in the continuous active log (as indicated by a blank in the last three columns) as recorded by the hand-held unit, indicating no loss of signal during the duration of the experiment.

The data obtained from the wearable GPS data-logger is presented in Table 21.

Table 20 - Data Obtained from Hand-Held GPS Unit for Effect of Heavy Rain on Availability of Satellite Signal Experiment

\begin{tabular}{|c|c|c|c|c|}
\hline Longitude & Alt(m) Date Time(GMT) & S secon & ids & $\mathrm{m} / \mathrm{s}$ \\
\hline T N39.647125 W079.974375 & $323.19 / 9 / 200420: 23: 120$ & 5 & 4 & 0.8 \\
\hline T N39.647297 W079.974567 & 328.9 9/9/2004 20:23:310 & 24 & 29 & 1.3 \\
\hline T N39.647383 W079.974933 & 326.5 9/9/2004 20:23:54 0 & 47 & 62 & 1.4 \\
\hline T N39.647447 W079.975256 & 324.6 9/9/2004 20:24:130 & 66 & 90 & 1.5 \\
\hline T N39.647469 W079.975747 & 324.6 9/9/2004 20:24:42 0 & 95 & 133 & 1.5 \\
\hline T N39.647428 W079.976219 & 324.6 9/9/2004 20:25:07 0 & 120 & 173 & 1.6 \\
\hline T N39.647469 W079.976542 & $323.19 / 9 / 200420: 25: 260$ & 139 & 201 & 1.5 \\
\hline T N39.647578 W079.976972 & $320.39 / 9 / 200420: 25: 510$ & 164 & 240 & 1.6 \\
\hline T N39.647619 W079.977378 & 319.3 9/9/2004 20:26:13 0 & 186 & 275 & 1.6 \\
\hline T N39.647642 W079.977700 & 318.8 9/9/2004 20:26:34 0 & 207 & 303 & 1.3 \\
\hline T N39.647642 W079.977722 & 318.3 9/9/2004 20:26:42 0 & 215 & 305 & 0.2 \\
\hline T N39.647706 W079.978000 & 318.3 9/9/2004 20:27:02 0 & 235 & 329 & 1.2 \\
\hline T N39.647769 W079.978108 & 318.3 9/9/2004 20:27:20 0 & 253 & 341 & 0.6 \\
\hline T N39.647769 W079.978539 & 317.9 9/9/2004 20:27:44 0 & 277 & 378 & 1.5 \\
\hline T N39.647728 W079.978772 & 316.9 9/9/2004 20:27:58 0 & 291 & 398 & 1.5 \\
\hline T N39.647706 W079.978772 & $318.39 / 9 / 200420: 28: 150$ & 308 & 401 & 0.1 \\
\hline T N39.647706 W079.978772 & 318.8 9/9/2004 20:28:16 0 & 309 & 401 & 0.0 \\
\hline
\end{tabular}

The sample data shows that there was no loss of signal since there are no V's in the second column. Even when the entire data set for the duration of experiment was 
analyzed, only one ' $\mathrm{V}$ ' was noted, which was less than 5 seconds. This entry in the data can be assumed to be insignificant since the each entry is recorded at an interval of 5 seconds.

Table 21 - Data Obtained from Wearable GPS Data-Logger Unit for Effect of Heavy Rain on Availability of Satellite Signal Experiment

\begin{tabular}{|l|}
\hline RECORD,FLAG,DATE,TIME,LAT,LONG,SPEED,HEAD,ALTITUDE,HDOP,SATS \\
\hline 66,A,9/9/2004,15:24:56,39.64755,-79.97722,1.4,278,1050,2.1,6 \\
\hline 67,A,9/9/2004,15:25:01,39.64757,-79.9773,1.4,277,1050,2.4,6 \\
\hline 68,A,9/9/2004,15:25:06,39.64758,-79.97737,1.5,278,1053,2.2,6 \\
\hline 69,A,9/9/2004,15:25:11,39.6476,-79.97743,2,274,1059,2,6 \\
\hline 70,A,9/9/2004,15:25:16,39.6476,-79.97752,1.9,262,1059,2.7,6 \\
\hline 71,A,9/9/2004,15:25:21,39.6476,-79.9776,2.1,265,1059,2.9,6 \\
\hline 72,A,9/9/2004,15:25:26,39.6476,-79.9777,1.8,264,1063,2.8,6 \\
\hline 73,A,9/9/2004,15:25:31,39.6476,-79.9778,2,264,1069,3.2,6 \\
\hline $74, \mathrm{~A}, 9 / 9 / 2004,15: 25: 36,39.64763,-79.97788,1.9,277,1069,1.9,6$ \\
\hline $75, \mathrm{~A}, 9 / 9 / 2004,15: 25: 41,39.64767,-79.97795,1.8,284,1069,1.8,6$ \\
\hline $76, \mathrm{~A}, 9 / 9 / 2004,15: 25: 46,39.64768,-79.97805,1.3,281,1069,2.3,6$ \\
\hline $77, \mathrm{~A}, 9 / 9 / 2004,15: 25: 51,39.6477,-79.97813,1.4,285,1069,2.4,6$ \\
\hline $78, \mathrm{~A}, 9 / 9 / 2004,15: 25: 56,39.64772,-79.97823,2,279,1069,2.3,6$ \\
\hline $79, \mathrm{~A}, 9 / 9 / 2004,15: 26: 01,39.64772,-79.97833,2.2,279,1066,2.7,6$ \\
\hline $80, \mathrm{~A}, 9 / 9 / 2004,15: 26: 06,39.64775,-79.97842,1.9,286,1066,1.9,6$ \\
\hline $81, \mathrm{~A}, 9 / 9 / 2004,15: 26: 11,39.64778,-79.97848,1.9,290,1066,1.9,6$ \\
\hline $82, \mathrm{~A}, 9 / 9 / 2004,15: 26: 16,39.64782,-79.97857,2,291,1069,2,6$ \\
\hline $83, \mathrm{~A}, 9 / 9 / 2004,15: 26: 21,39.64785,-79.97865,2,292,1069,2,6$ \\
\hline
\end{tabular}

Thus, analysis of collected data shows that a very heavy rain did not affect the availability of signal to the two GPS units. Note, however, that this experiment involved only a single run under one particular (and unquantified) rainfall intensity. On this basis, it cannot be concluded that the GPS units would perform equally well in even heavier rains or other weather circumstances such as heavy snow and thick fog. Resource constraints did not permit an assessment of a full range of weather conditions. Thus, further research is recommended in performing similar experiments in varying weather conditions to ascertain their effect on the utility of devices.

\subsubsection{Signal Availability in the Vicinity of Tall Buildings}

The experiment was carried out over two days at three different locations, namely, on West Virginia University’s Evansdale Campus, in downtown Morgantown, WV and 
in downtown Pittsburgh, PA. The weather on each of the days of experiment was similar and tests were carried out during daylight when there was no falling precipitation (partly cloudy with temperatures varying from $60 \mathrm{~F}$ to $73 \mathrm{~F}$ ). The procedure described in Chapter 3 was followed for the start-up of the two GPS units at all locations. The two units were switched on as the researcher approached the test locations

Experiments at the first location, i.e. between the NRCCE building and the Evansdale Library on the Evansdale Campus of West Virginia University were carried out in mid-September in the late afternoon. The hand-held GPS unit took about two minutes to acquire a good signal from the satellites and to configure itself. The unit showed a strong signal from 4 satellites (and a weak signal from one more) and the accuracy was 18 feet. After walking between the buildings for about 10 minutes, no significant change in the satellite signal or the accuracy was observed. The number of satellites shown on the display screen fluctuated between 4 and 6 and the accuracy varied from 15 feet to 22 feet.

The data collected from the hand-held GPS unit, after downloading it to a computer, is shown in Table 22. As can be seen from the sample data shown in Table 22, there were no gaps between the logs for the last three columns, indicating that the unit was in constant communication with the satellites and thus recorded readings at regular intervals.

Table 22 - Data Obtained from Hand-held GPS Unit at Site 1, for Effect of Tall Buildings on Availability of Satellite Signal Experiment

\begin{tabular}{|c|c|c|c|c|c|}
\hline F Latitude & Longitude & n) Date Time(GMT) $\mathrm{S} \mathrm{sec}$ & ads & $\mathrm{m} / \mathrm{s}$ & \\
\hline \multicolumn{2}{|c|}{ T N39³8'45.33" W07958'17.71" } & $341.49 / 21 / 200422: 41: 510$ & 290 & 210 & 0.5 \\
\hline \multicolumn{2}{|c|}{ T N39³8'45.25" W07958'18.02" } & $343.39 / 21 / 200422: 42: 580$ & 357 & 218 & 0.1 \\
\hline \multicolumn{2}{|c|}{ T N39³8'45.02" W07958'17.94" } & $341.99 / 21 / 200422: 44: 350$ & 454 & 225 & 0.1 \\
\hline \multicolumn{2}{|c|}{ T N39॰38'45.02" W07958'17.94" } & $341.49 / 21 / 200422: 44: 470$ & 466 & 225 & 0.0 \\
\hline \multicolumn{2}{|c|}{ T N39³8'45.02" W07958'17.94" } & $341.49 / 21 / 200422: 45: 490$ & 528 & 225 & 0.0 \\
\hline \multicolumn{2}{|c|}{ T N39॰38'45.10" W07958'18.32" } & $341.49 / 21 / 200422: 47: 370$ & 636 & 235 & 0.1 \\
\hline \multicolumn{2}{|c|}{ T N39³8'45.10" W07958'18.48" } & $344.89 / 21 / 200422: 48: 340$ & 693 & 239 & 0.1 \\
\hline
\end{tabular}




\begin{tabular}{|c|c|c|c|c|}
\hline T N39³8'44.94" W07958'18.71" & $344.89 / 21 / 200422: 48: 380$ & 697 & 246 & 1.8 \\
\hline T N39³ $38^{\prime} 44.25^{\prime \prime}$ W0795 $58^{\prime} 18.48^{\prime \prime}$ & $344.89 / 21 / 200422: 48: 530$ & 712 & 268 & 1.5 \\
\hline T N39³8'43.32" W07958'18.09" & $343.89 / 21 / 200422: 49: 130$ & 732 & 298 & 1.5 \\
\hline T N39³8'41.93" W07958'18.09" & 346.2 9/21/2004 22:49:46 0 & 765 & 341 & 1.3 \\
\hline T N39³8'41.70" W07958'18.32" & 345.7 9/21/2004 22:49:55 0 & 774 & 350 & 1.0 \\
\hline $\mathrm{T}^{\prime}$ N39³8'41.54" W07958'18.71" & $344.39 / 21 / 200422: 50: 020$ & 781 & 361 & 1.5 \\
\hline T N39³8'41.70" W07958'18.94" & $343.39 / 21 / 200422: 50: 070$ & 786 & 368 & 1.5 \\
\hline $\mathrm{T}^{2} 39^{\circ} 38^{\prime} 42.86^{\prime \prime} \mathrm{W} 079^{\circ} 58^{\prime} 20.26^{\prime \prime}$ & $342.49 / 21 / 200422: 50: 410$ & 820 & 415 & 1.4 \\
\hline 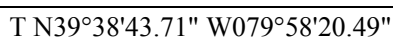 & $341.49 / 21 / 200422: 50: 570$ & 836 & 442 & 1.7 \\
\hline T N39³8'44.63" W07958'19.48" & $343.39 / 21 / 200422: 51: 330$ & 872 & 479 & 1.0 \\
\hline T N39³8'44.63" W07958'19.48" & $343.39 / 21 / 200422: 51: 340$ & 873 & 479 & 0.0 \\
\hline T N39³8'43.94" W07958'20.41" & $343.39 / 21 / 200422: 51: 580$ & 897 & 510 & 1.3 \\
\hline T N39³8'42.78" W07958'21.26" & $342.49 / 21 / 200422: 52: 260$ & 925 & 551 & 1.5 \\
\hline $\mathrm{T}^{2} 39^{\circ} 38^{\prime} 42.55^{\prime \prime} \mathrm{W} 079^{\circ} 58^{\prime} 21.26^{\prime \prime}$ & $341.49 / 21 / 200422: 52: 450$ & 944 & 558 & 0.4 \\
\hline
\end{tabular}

For the wearable unit, readings were recorded at a uniform interval of 5 seconds.

Sample data shown in Table 23 indicate that the unit was receiving a signal from 5 to 7

satellites all along the duration of the experiment. This can be inferred from the fact that there are no 'V's under the column for flag.

Table 23 - Data Obtained from Wearable GPS unit at Site 1 for Effect of Tall Buildings on Availability of Satellite Signal Experiment

\begin{tabular}{|l|}
\hline RECORD,FLAG,DATE,TIME,LAT,LONG,SPEED,HEAD,ALTITUDE,HDOP,SATS \\
\hline $73, \mathrm{~A}, 09 / 21 / 04,18: 39: 35,39.64597,-79.972,002.1,115,01161,03.0,05$ \\
\hline $74, \mathrm{~A}, 09 / 21 / 04,18: 39: 39,39.64595,-79.97195,002.2,119,01158,03.0,05$ \\
\hline $75, \mathrm{~A}, 09 / 21 / 04,18: 39: 45,39.64592,-79.9719,002.2,123,01158,02.8,05$ \\
\hline $76, \mathrm{~A}, 09 / 21 / 04,18: 39: 49,39.64590,-79.97185,002.2,125,01161,02.8,05$ \\
\hline $77, \mathrm{~A}, 09 / 21 / 04,18: 39: 54,39.64588,-79.97183,001.7,127,01161,02.0,06$ \\
\hline $78, \mathrm{~A}, 09 / 21 / 04,18: 39: 59,39.64588,-79.97183,001.1,126,01161,02.0,06$ \\
\hline $79, \mathrm{~A}, 09 / 21 / 04,18: 40: 05,39.64588,-79.97183,000.0,126,01161,02.1,06$ \\
\hline $80, \mathrm{~A}, 09 / 21 / 04,18: 40: 09,39.64588,-79.97182,000.0,126,01158,01.8,07$ \\
\hline $81, \mathrm{~A}, 09 / 21 / 04,18: 40: 14,39.64588,-79.9718,000.0,126,01158,01.9,07$ \\
\hline $82, \mathrm{~A}, 09 / 21 / 04,18: 40: 19,39.64588,-79.97182,000.0,126,01158,02.0,07$ \\
\hline $83, \mathrm{~A}, 09 / 21 / 04,18: 40: 24,39.64588,-79.97183,000.0,126,01161,02.1,07$ \\
\hline $84, \mathrm{~A}, 09 / 21 / 04,18: 40: 29,39.64590,-79.9718,000.0,126,01155,01.8,07$ \\
\hline $85, \mathrm{~A}, 09 / 21 / 04,18: 40: 34,39.64590,-79.9718,000.0,126,01151,01.8,07$ \\
\hline $86, \mathrm{~A}, 09 / 21 / 04,18: 40: 39,39.64590,-79.9718,000.0,126,01151,01.8,07$ \\
\hline $87, \mathrm{~A}, 09 / 21 / 04,18: 40: 44,39.64590,-79.9718,000.0,126,01151,01.7,07$ \\
\hline $88, \mathrm{~A}, 09 / 21 / 04,18: 40: 49,39.64592,-79.97178,000.0,126,01151,01.7,07$ \\
\hline $89, \mathrm{~A}, 09 / 21 / 04,18: 40: 54,39.64590,-79.9718,000.0,126,01155,01.8,07$ \\
\hline $90, \mathrm{~A}, 09 / 21 / 04,18: 41: 00,39.64592,-79.9718,000.0,126,01155,01.7,07$ \\
\hline $91, \mathrm{~A}, 09 / 21 / 04,18: 41: 04,39.64592,-79.9718,000.0,126,01155,01.9,07$ \\
\hline $92, \mathrm{~A}, 09 / 21 / 04,18: 41: 09,39.64590,-79.9718,000.0,126,01155,01.7,07$ \\
\hline
\end{tabular}

The second test location (site 2) was the central business district (CBD) of

Morgantown. The experiment was carried out in mid-September in the morning. The

hand-held GPS unit took about three minutes to acquire a good signal from the satellites 
and to configure itself. The unit showed a strong signal from 5 satellites (and a weak signal from one more) and the accuracy was 17 feet.

Satellite signals displayed on the screen changed rapidly. Table 24 shows a sample from the data recording sheet for the number of satellite signals and the accuracy at that instant, recorded at various times during the experiment from the display screen of the hand-held GPS unit.

Table 24 - Data from Recording Sheet at Site 2 for Effect of Tall Buildings on Availability of Satellite Signal Experiment

\begin{tabular}{|c|c|c|c|}
\hline & No. of Satellite Signals on Display Screen & \\
\hline Clock Time & Strong Signal & Weak Signal & Accuracy in feet \\
\hline $10: 45$ a.m. & 5 & 1 & 17 \\
\hline $10: 45$ a.m. & 4 & 1 & 21 \\
\hline $10: 45$ a.m. & 2 & 1 & 48 \\
\hline $10: 45$ a.m. & "Need clear view of Sky" & 63 \\
\hline $10: 51$ a.m. & 2 & 0 & 49 \\
\hline $10: 52$ a.m. & 3 & 0 & 51 \\
\hline $10: 53$ a.m. & "Need clear view of Sky" & 42 \\
\hline $10: 55$ a.m. & 3 & 0 & 29 \\
\hline $10: 55$ a.m. & 3 & 1 & 20 \\
\hline $10: 55$ a.m. & 4 & 1 & \\
\hline $10: 57$ a.m. & "Need clear view of Sky" & \\
\hline $11: 01$ a.m. & 4 & 1 & \\
\hline
\end{tabular}

As shown in Table 24, there were 3 relatively long periods of very weak or no signal during the experiment and the display screen showed the message "Need Clear View of Sky". The hand-held GPS unit takes some time to lock into the signals of the satellites. If it is unable to track three satellites after approximately one minute, it displays a message indicating the need for a clear view of sky.

A sample of the data obtained after connecting the hand-held GPS unit to a computer is shown in Table 25. Table 25 shows that there were 3 periods of considerable duration when there was no signal available from the satellites. The gap in the otherwise continuous log shows a loss of signal. 
Table 25 - Data Obtained from Hand-Held GPS Unit at Site 2 for Effect of Tall Buildings on Availability of Satellite Signal Experiment

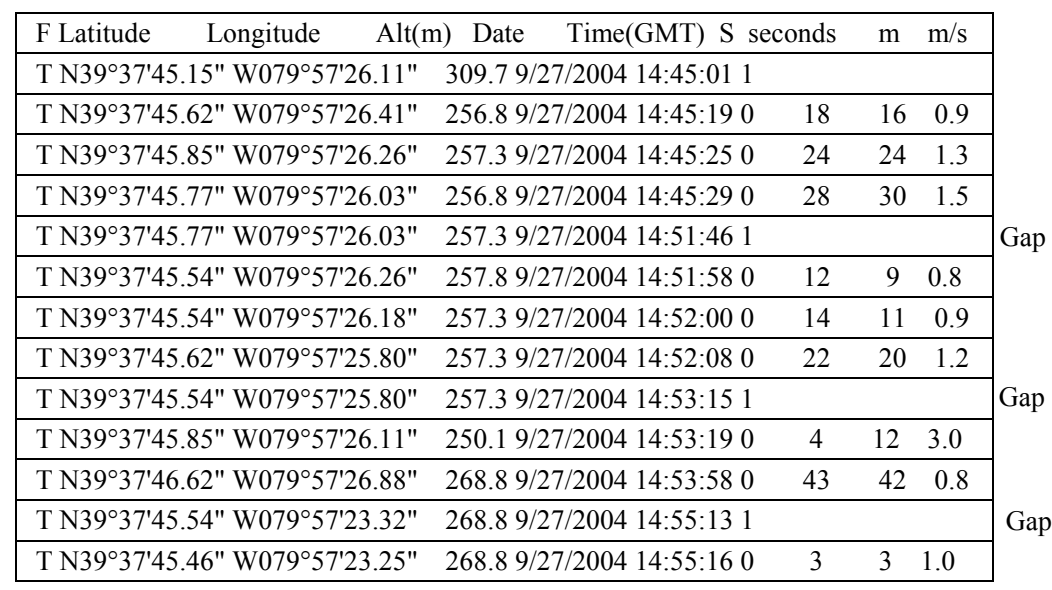

As seen in Table 25, in one case, there was no signal for almost 6 minutes. Such a lengthy period of no signal can affect the reliability of the data collected since during the period of no signal, no information is recorded and the points obtained are not continuous. For example, the time gap between the time recorded at the first gap and the time of the previous entry is approximately 6 minutes. During this time, no entries were recorded. This means that the location of the user is lost for that period of time when there is no signal from satellites for the device. This, in terms of pedestrian data collection would mean that the user's position is unknown for that period of time. Obviously, this is a severe limitation of the device.

A sample of the data obtained at the same location (i.e., site 2) from the wearable GPS data-logger is shown in Table 26. The second column in Table 26 shows a relatively large number of V's (indicating signal loss). The last column of the data shows the number of satellites to which the unit is connected. Note that, in this case, there are a number of zero entries, indicating that unit was not in contact with any satellites. The position of the subject could be determined for the period of time in which there was no signal, but this position data may not be reliable. 
Table 26 - Data Obtained from Wearable GPS Unit at Site 2 for Effect of Tall Buildings on Availability of Satellite Signal Experiment

\begin{tabular}{|l|}
\hline RECORD,FLAG,DATE,TIME,LAT,LONG,SPEED,HEAD,ALTITUDE,HDOP,SATS \\
\hline $36, \mathrm{~A}, 09 / 27 / 04,10: 45: 30,39.62935,-79.95715,001.9,074,00928,01.9,07$ \\
\hline $37, \mathrm{~A}, 09 / 27 / 04,10: 45: 35,39.62933,-79.9571,002.1,100,00928,02.5,07$ \\
\hline $38, \mathrm{~A}, 09 / 27 / 04,10: 45: 40,39.62932,-79.95705,002.2,106,00938,02.5,07$ \\
\hline $39, \mathrm{~A}, 09 / 27 / 04,10: 45: 45,39.62933,-79.957,002.2,107,00938,11.8,06$ \\
\hline $40, \mathrm{~V}, 09 / 27 / 04,10: 46: 06,39.62930,-79.95673,000.0,000,00945,12.2,00$ \\
\hline $41, \mathrm{~V}, 09 / 27 / 04,10: 46: 57,39.62872,-79.9573,000.0,000,00951,04.4,00$ \\
\hline $42, \mathrm{~V}, 09 / 27 / 04,10: 47: 00,39.62905,-79.95713,000.0,000,00951,04.4,00$ \\
\hline $43, \mathrm{~V}, 09 / 27 / 04,10: 47: 48,39.62927,-79.95667,000.0,000,00951,04.6,00$ \\
\hline $44, \mathrm{~V}, 09 / 27 / 04,10: 48: 05,39.62918,-79.95678,000.0,000,00951,02.1,00$ \\
\hline $45, \mathrm{~V}, 09 / 27 / 04,10: 48: 10,39.62912,-79.9568,000.0,000,00951,02.5,00$ \\
\hline $46, \mathrm{~V}, 09 / 27 / 04,10: 48: 15,39.62930,-79.9567,000.0,107,00951,04.5,04$ \\
\hline $47, \mathrm{~V}, 09 / 27 / 04,10: 48: 20,39.62918,-79.95678,000.0,000,00951,04.5,00$ \\
\hline $48, \mathrm{~V}, 09 / 27 / 04,10: 48: 26,39.62912,-79.95682,000.0,000,00951,04.5,00$ \\
\hline $49, \mathrm{~A}, 09 / 27 / 04,10: 48: 30,39.62908,-79.95682,000.0,107,00951,04.5,03$ \\
\hline $50, \mathrm{~V}, 09 / 27 / 04,10: 50: 13,39.62945,-79.95688,000.0,000,00951,05.2,00$ \\
\hline $51, \mathrm{~V}, 09 / 27 / 04,10: 50: 17,39.62880,-79.95857,000.0,000,00951,23.2,00$ \\
\hline $52, \mathrm{~A}, 09 / 27 / 04,10: 50: 20,39.62955,-79.95685,000.0,000,00951,23.0,04$ \\
\hline $53, \mathrm{~A}, 09 / 27 / 04,10: 50: 25,39.62958,-79.95698,000.0,236,01007,02.2,04$ \\
\hline $54, \mathrm{~A}, 09 / 27 / 04,10: 50: 30,39.62963,-79.95708,000.0,236,01017,01.8,05$ \\
\hline $55, \mathrm{~V}, 09 / 27 / 04,10: 50: 35,39.62962,-79.95707,000.0,236,00991,02.0,05$ \\
\hline
\end{tabular}

The third location (site 3) was located in downtown Pittsburgh, PA. This location

had tall buildings (tens of stories high), located close to each other. Table 27 presents sample data for hand-held GPS unit.

Table 27 - Data Obtained from Hand-held GPS Unit at Site 3 for Effect of Tall Buildings on Availability of Satellite Signal Experiment

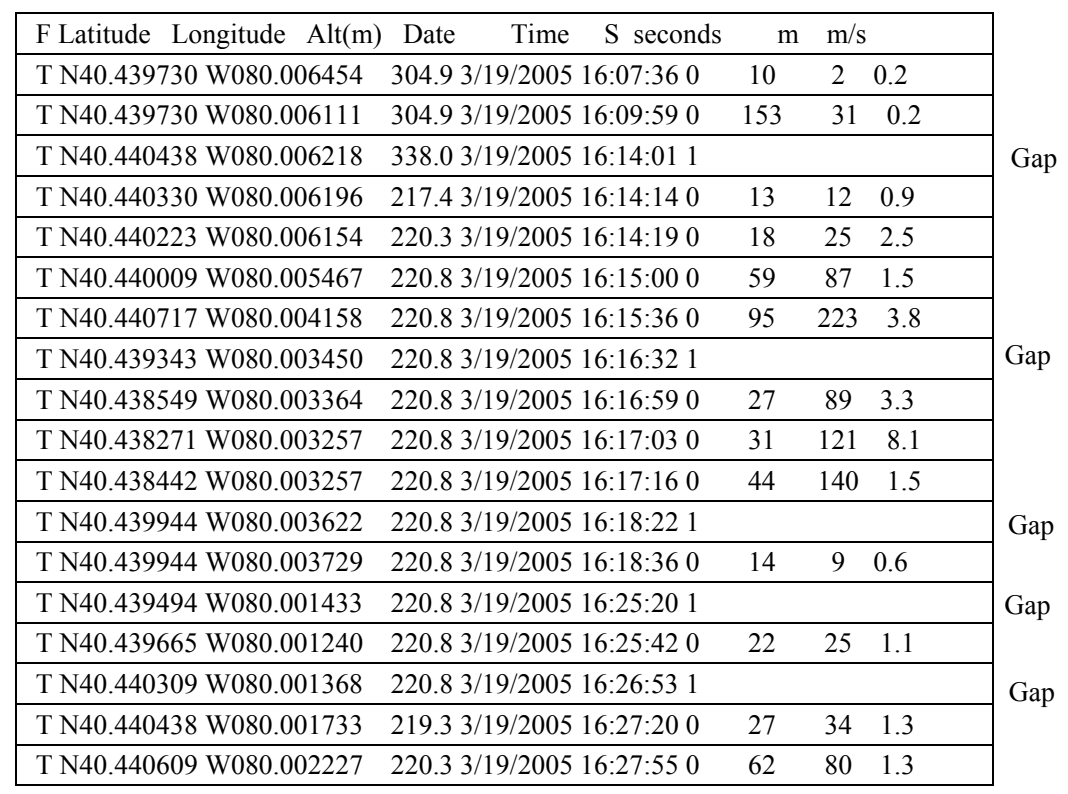


As evident from the gaps in the continuous active log, the unit lost satellite signal a number of times, and in each instance, for a significant period of time. The duration of time without signal varied from 13 seconds to 71 seconds. Sample data recorded and obtained from the wearable GPS data-logger unit are shown in Table 28.

Table 28 - Data Obtained from Wearable GPS Unit at Site 3 for Effect of Tall Buildings on Availability of Satellite Signal Experiment

\begin{tabular}{|l|}
\hline RECORD,FLAG,DATE,TIME,LAT,LONG,SPEED,HEAD,ALTITUDE,HDOP,SATS \\
\hline $269, \mathrm{~A}, 03 / 19 / 05,11: 29: 48,40.44100,-80.0038,002.7,292,00764,03.6,03$ \\
\hline $270, \mathrm{~V}, 03 / 19 / 05,11: 29: 53,40.44105,-80.00387,002.8,290,00761,03.0,04$ \\
\hline $271, \mathrm{~A}, 03 / 19 / 05,11: 29: 58,40.44112,-80.00392,000.0,000,00754,04.6,04$ \\
\hline $272, \mathrm{~V}, 03 / 19 / 05,11: 30: 03,40.44113,-80.004,000.0,000,00000,00.0,00$ \\
\hline $273, \mathrm{~V}, 03 / 19 / 05,11: 30: 11,40.44033,-80.00622,000.0,000,00771,02.9,04$ \\
\hline $274, \mathrm{~V}, 03 / 19 / 05,11: 30: 34,40.45815,-80.01258,000.0,000,00771,28.2,00$ \\
\hline $275, \mathrm{~V}, 03 / 19 / 05,11: 30: 38,40.44062,-80.00347,000.0,000,00797,02.2,00$ \\
\hline $276, \mathrm{~V}, 03 / 19 / 05,11: 30: 46,40.44000,-80.00642,000.0,000,00000,00.0,00$ \\
\hline $277, \mathrm{~V}, 03 / 19 / 05,11: 30: 51,40.43923,-80.00353,000.0,000,00820,05.2,00$ \\
\hline $278, \mathrm{~V}, 03 / 19 / 05,11: 30: 54,40.43943,-80.00632,000.0,000,00817,03.0,00$ \\
\hline $279, \mathrm{~V}, 03 / 19 / 05,11: 30: 59,40.43810,-80.00332,000.0,000,00830,05.3,00$ \\
\hline $280, \mathrm{~V}, 03 / 19 / 05,11: 31: 03,40.44042,-80.00413,000.0,291,00830,02.8,00$ \\
\hline $281, \mathrm{~A}, 03 / 19 / 05,11: 31: 08,40.44058,-80.00467,002.0,181,00899,05.3,05$ \\
\hline $282, \mathrm{~V}, 03 / 19 / 05,11: 31: 14,40.44105,-80.005,000.0,000,00912,05.3,00$ \\
\hline $283, \mathrm{~A}, 03 / 19 / 05,11: 31: 18,40.44093,-80.00475,000.0,000,00918,04.8,05$ \\
\hline $284, \mathrm{~A}, 03 / 19 / 05,11: 31: 23,40.44092,-80.00465,000.0,000,00918,03.4,05$ \\
\hline $285, \mathrm{~V}, 03 / 19 / 05,11: 31: 28,40.44208,-80.00355,001.5,198,00918,03.4,05$ \\
\hline $286, \mathrm{~A}, 03 / 19 / 05,11: 31: 33,40.43400,-80.00322,000.0,000,00918,00.0,05$ \\
\hline $287, \mathrm{~V}, 03 / 19 / 05,11: 31: 38,40.44012,-80.00387,002.1,178,00925,04.9,05$ \\
\hline $288, \mathrm{~A}, 03 / 19 / 05,11: 31: 43,40.43953,-80.0035,001.1,180,00925,04.9,04$ \\
\hline $289, \mathrm{~V}, 03 / 19 / 05,11: 31: 50,40.44072,-80.0038,000.0,000,00922,04.9,00$ \\
\hline $290, \mathrm{~A}, 03 / 19 / 05,11: 31: 53,40.44070,-80.00405,001.8,184,00945,02.0,05$ \\
\hline $291, \mathrm{~V}, 03 / 19 / 05,11: 33: 00,40.44047,-80.00483,000.0,000,00948,07.4,00$ \\
\hline $292, \mathrm{~A}, 03 / 19 / 05,11: 33: 03,40.43890,-80.0057,002.8,300,00469,04.1,03$ \\
\hline $293, \mathrm{~A}, 03 / 19 / 05,11: 33: 08,40.43873,-80.00555,001.2,322,00318,04.2,04$ \\
\hline
\end{tabular}

The wearable GPS data logger unit also lost the satellite signal frequently as is evident from the data shown in Table 28. However, it had a better connectivity in comparison to the hand-held GPS unit. Better connectivity here refers to stronger capability in acquiring satellite signal. Among the records shown in Table 28, significant numbers of entries show zero satellite signals. This data, if plotted on a map, would not show actual position of the wearable GPS data-logger user, and thus not be reliable. For example, if recorded data is plotted for a pedestrian who had been carrying the wearable GPS data-logger unit, and the data recorded involved entries with zero satellite signals, 
the position of the user will not be determined correctly for the period when there were zero signals.

From the experiments conducted between tall buildings, it was determined that the two GPS units worked well in recording data between buildings on the order of 2 to 3 stories tall. It was also observed that acquiring satellite signals, and hence recording data, became more difficult for both GPS units as building height increased and spacing between buildings decreased. In using the two GPS units between tall buildings to check for availability of satellite signal, it was observed that, for two to three story buildings, the GPS devices connected to the satellites without difficulty. This was concluded from the analysis of recorded data. The wearable GPS data logger showed slow initial connectivity to satellites, but later recorded data after constant intervals as expected. For buildings taller than 3 stories, both units had problems locating the satellites and even when they were connected, the signals were either weak or less than the required 4 signals. Using the two units in close proximity to tall buildings did not give reliable results as the building affected the ability of the devices to acquire satellite signals. This is the so-called urban canyon effect. There were many instances when both units were without any signal for a considerable period of time. It was observed that the wearable GPS data-logger was more capable in connecting with satellites and thus more efficient in collecting data compared to the hand-held GPS unit.

For data collection purposes, this loss of signal may be a significant problem as the data obtained will not be continuous and reliable and will not give the correct position of the user. In addition to position, other travel data such as speed, location and altitude will also not be reliable as the accuracy goes down with fewer satellites. When the GPS 
units do not receive any signal, there will be no entries recorded in the memory. These entries give various travel information about the user such as position, speed and time of day. Thus, further research is recommended to find a solution in overcoming the loss of signal as a result of the urban canyon effect.

Overall Assessment of the Hand-Held GPS Unit and the Wearable GPS Data-Logger

Assessing the hand-held GPS unit and the wearable GPS data-logger after analyzing them under 4 different field conditions showed mixed results. Both units worked well under the high-voltage transmission lines and next to transmission towers. Under high-voltage facilities, no effect was observed on the availability of satellite signal, and data was recorded constantly. Similarly, limited testing showed that heavy rainfall did not affect the data recording capabilities of the two devices. For the hand-held GPS unit under trees, there were a few instances where it lost connectivity to the satellites for significant periods of time and this loss of signal was not evident from the display screen. The wearable GPS data-logger unit had no problems connecting to satellites under dense tree canopy for both deciduous and coniferous trees. When used near closely spaced buildings taller than 2 or 3 stories, both units seem to have a problem locating the satellites and in many instances data were not recorded for a significant time period.

Based upon the analysis of the data collected from the two GPS devices under various experimental conditions, it was concluded that the two GPS devices can be used for collecting pedestrian travel data with certain restrictions. These restrictions involve various field conditions where the data collected may not prove to be fully reliable, for example, in urban canyons (closely spaced tall buildings) and under thick forest covers. The same devices, when used in suburban or rural locations should provide reliable 
pedestrian travel data. Reliable travel data can be obtained only when there is direct communication between the GPS devices and satellites. Additional research is recommended to study the interaction effect of field conditions such as effect of highvoltage power lines along with heavy precipitation on the signal availability, or under trees covered with heavy snow. Research is also required to ascertain the effect of terrain with high relief on the availability of satellite signal. Effects of extreme weather conditions on signal availability also needs more study.

\subsection{Evaluation of Operational Considerations - Pedometer}

Following brief instructions, the wearable pedometer and travel log form were given to five subjects for periods of 24 hours each. The data collected using the pedometer for all subjects, for all trips was collected for analysis. Along with the pedometer, the subjects also returned their completed travel data log form which provided information about their non-motorized trips. When they returned the pedometer and travel $\log$ forms, they were asked to complete a brief questionnaire about the experience. The data from the five subjects was compiled and analyzed to determine various types of trip information such as origin and destination, trip duration, trip distance and speed. The data analysis procedure was described in Chapter 3.

Since the pedometer had no data recording capability, the only data available for the study was from the travel $\log$ forms. From the travel log form given to each subject, various trip information such as street address and time at origin, street address and time at destination, mode of travel and number of steps was obtained for all non-motorized trips made during the duration of experiment for all subjects. 
Table 29 presents a summary of the information obtained from trip logs. The table includes information such as number of trips made by each subject, total number of steps, average number of steps for each trip, total and average distance walked and average duration of each trip. Analysis of the trip logs obtained from each subject after completion of the experiment permitted a number of observations to be made. It was observed that the subjects made seven to twelve walking trips outdoors in the 24-hour study period. The average trip duration ranged from 8 minutes to almost 15 minutes. The total distance walked by the subjects during the duration of experiment varied from 2.5 miles to 4.5 miles whereas the average distance for each trip for the subjects varied from 0.3 miles to 0.6 miles. Considering the grand averages, the average number of trips was 9 walking trips and the average trip time was 10 minutes. The average total distance walked was 3.4 miles whereas average distance walked per trip was 0.4 miles.

Table 29 - Summary of Trip Information Obtained from Subjects in Pedometer Experiment

\begin{tabular}{|c|c|c|c|c|c|c|}
\hline \multirow{2}{*}{ Subject } & $\begin{array}{c}\text { No. of } \\
\text { Walking } \\
\text { Trips }\end{array}$ & $\begin{array}{c}\text { Total } \\
\text { Number } \\
\text { of Steps }\end{array}$ & $\begin{array}{c}\text { Total } \\
\text { Distance } \\
\text { Walked } \\
\text { (miles) }\end{array}$ & $\begin{array}{c}\text { Average } \\
\text { Number } \\
\text { of Steps } \\
\text { per Trip }\end{array}$ & $\begin{array}{c}\text { Average } \\
\text { Distance } \\
\text { Walked/Trip } \\
\text { (miles) }\end{array}$ & $\begin{array}{c}\text { Average } \\
\text { Duration of } \\
\text { Trips (min) }\end{array}$ \\
\hline 1 & 12 & 7836 & 3.6 & 653 & 0.3 & 8.6 \\
\hline & & & & & & \\
\hline & 8 & 8760 & 4 & 1095 & 0.5 & 10.3 \\
\hline 3 & 8 & 9888 & 4.5 & 1236 & 0.6 & 14.6 \\
\hline 4 & 9 & & & & & \\
\hline & & 5787 & 2.6 & 643 & 0.3 & 8 \\
\hline 5 & 7 & 5488 & 2.5 & 784 & 0.3 & 8.3 \\
\hline
\end{tabular}

The minimum and maximum duration for any trip as observed after analysis of the trip logs was 2 minutes and 32 minutes, respectively. Also, the minimum and the 
maximum number of steps required for any trip was 207 and 3413, respectively, which represents approximate distances of 0.1 mile and 1.6 mile, respectively.

A typical travel log form with the data recorded by one of the subjects is shown in Table 30. Ideally, the travel $\log$ should be presented verbatim, but in order to insure privacy of the subjects, and to give a better description of the origins and destinations, the travel $\log$ presented here is the version edited by the researcher.

Table 30 - Data Recorded by Subject 4 in the Travel Log Form for Pedometer Experiment

\begin{tabular}{|c|c|}
\hline Trip 1 & Trip 6 \\
\hline Origin- Home & Origin- Towers PRT \\
\hline Origin time $-8: 45 \mathrm{am}$ & Origin time $-1: 05 \mathrm{pm}$ \\
\hline Destination- Towers Residence Hall & Destination- Towers Residence Hall \\
\hline Destination time- $8: 51 \mathrm{am}$ & Destination time- $1: 13 \mathrm{pm}$ \\
\hline Mode - Walk & Mode - Walk \\
\hline Trip 2 & Trip 7 \\
\hline Origin- Towers Residence Hall & Origin- Towers Residence Hall \\
\hline Origin time $-11: 05 \mathrm{am}$ & Origin time $-3: 30 \mathrm{pm}$ \\
\hline Destination- Medical Center Apts. & Destination- Kroger \\
\hline Destination time- 11:17 am & Destination time- $3: 37 \mathrm{pm}$ \\
\hline Mode - Walk & Mode - Walk \\
\hline Trip 3 & Trip 8 \\
\hline Origin- Medical Center Apts. & Origin- Kroger \\
\hline Origin time $-11: 25 \mathrm{am}$ & Origin time $-3: 50 \mathrm{pm}$ \\
\hline Destination- Office Depot & Destination- Towers Residence Hall \\
\hline Destination time- 11:35 am & Destination time- 3:59 pm \\
\hline Mode - Walk & Mode - Walk \\
\hline Trip 4 & Trip 9 \\
\hline Origin- Beechurst PRT Station & Origin- Towers Residence Hall \\
\hline Origin time $-12: 10 \mathrm{pm}$ & Origin time $-5: 15 \mathrm{pm}$ \\
\hline Destination- Wise Library & Destination- Home \\
\hline Destination time- $12: 17 \mathrm{pm}$ & Destination time- 5:21 pm \\
\hline Mode - Walk & Mode - Walk \\
\hline Trip 5 & \\
\hline Origin- Wise Library & Origin- \\
\hline Origin time $-12: 35 \mathrm{pm}$ & Origin time - \\
\hline Destination- Beechurst PRT Station & Destination- \\
\hline Destination time- $12: 43 \mathrm{pm}$ & Destination time- \\
\hline Mode - Walk & Mode - \\
\hline
\end{tabular}

As can be observed from Table 30, the only data available for analysis of trips made by an individual is the travel log form which does look reasonable. The origins and destinations recorded can only be assumed to be accurate, i.e., relying on the subject for providing correct information. This is because the pedometer does not have any data 
recording capability (unlike the GPS devices) for position of the user. Thus, the only data available for analysis are the entries made in the travel log form which may or may not indicate the correct origins and destinations. They are susceptible to errors and omissions since the entries cannot be confirmed in any other way. Consequently, the trip purpose can only be assumed (not confirmed) by looking at the origins/destinations recorded in the travel log form.

The number of steps can be used to get a rough approximation of the distance traveled (along the route selected) between the origin and the destination by multiplying the number of steps times the subject's stride length. For example, consider Trip 2 made by subject 4: Origin- Towers Residence Hall on the Evansdale Campus of West Virginia University; Destination- Medical Center Apartments; Origin time- 11:05 a.m., Destination time- 11:17 a.m., Step count- 1012. The subject had a stride length of 2.4 feet (average stride length). Thus, it can be seen that the subject traveled a distance of 2.4 x $1012=2429$ feet $\sim 0.46$ miles. The speed of the subject during the trip is found to be $2429 /(12 \times 60)=3.37 \mathrm{fps} \sim 3.4 \mathrm{fps}=2.3 \mathrm{mph}$. Similarly, the distance traveled and the walking speed can be ascertained for each trip, for each subject. Note that the intermodal connections are not shown explicitly and the data obtained shows only the walking trips. It was inferred that there were at least two inter-modal trips involving motorized means of travel. For example, trip 3 ended at an office supply store in the Evansdale section of Morgantown. Trip 4 began at a people mover station near downtown, i.e. several miles away. Given the elapsed time, it can be reasonably concluded that the trip was not made by walking. The most likely mode was an automobile but this cannot be verified from the data. 
After analysis of the data obtained from the trip logs, a few inconsistencies/errors were observed. Two inconsistencies were observed for Subject 1- trip origins mentioned in the trip $\log$ for one of the trips seemed out of place (i.e. incorrect); for another of Subject 1's trips, no destination time was stated. These questions were reviewed (shortly after the logs were returned) with the subject to try to resolve the inconsistencies. For Subject 3, the step count for two trips seemed inconsistent with the geographic distances between the origins and destinations for those trips. This may be due to reasons such as improper closing of the pedometer or improper wearing of the pedometer on the waist. Another possible reason may be fabrication of the data by the subject. The subject was asked whether any of the stated reasons were true, but subject did not remember. Upon review, with Subject 4 of his trips, the subject mentioned that for two separate trips, he forgot to record the pedometer step count upon reaching the destination for those two trips, and the record was made after a small duration when he realized this error. This cannot be known from the obtained data. After all trips were analyzed for all the subjects, it was estimated that $14 \%$ of the total trips had questionable step counts.

The time required for analysis of the data to produce the data just discussed, obtained from the pedometer experiment, varied from an hour and a half to two hours for each subject, depending on the number of trips recorded by the subject. This includes analyzing both the travel log form and the response questionnaire, which would not be part of a normal work effort.

The technician or individual analyzing the data should have basic knowledge of math and should possess analytical skills in order to calculate the distances traveled and speed of the subject from the step count. The analyst should also understand how a 
pedometer works and how to wear them correctly, in order to identify errors in step count recorded on the travel log form. The analysis of data may also require knowledge of computers since if the technique is used on a large scale, a database or spreadsheet may be used for data compilation. Besides these qualifications, the analyst should have a general idea of the geographic location where the subject travels. The analyst should also possess good communication skills in order to clearly convey to the subject, the procedure to be followed and the value of the data.

After the subjects completed the experiment, they were asked to answer a few questions on their experience with the pedometer during the study. Table 31 presents a summary of the responses received for the questionnaire. The table also presents the comments made by the subjects while completing the questionnaire.

From Table 31, it can be seen that subjects unanimously reported they followed the correct procedure for data collection, as described in the instructions. Even though all subjects reported that they recorded all of their trips on the travel log form, the researcher determined that one subject omitted entries for some of the fields in the travel log. None of the subjects reported that they failed to reset the pedometer unit or forgot to carry the travel log form on their walking trips. This, of course, cannot be independently verified.

According to the survey form, only one subject felt that the pedometer was uncomfortable in that there was concern the device would fall off if the subject walked fast. This was an unusual concern and could indicate that the pedometer was not attached correctly or the subject's dress interfered with the positioning of the pedometer. A majority of subjects thought that it was bothersome to carry the travel $\log$ form and 
record all walking trips. It is somewhat surprising that the proportion feeling this way was not higher.

Table 31-Summary of Subject Responses to Questionnaire on Pedometer Study

\begin{tabular}{|c|c|c|c|c|}
\hline Question & Yes & No & Blank & Comments \\
\hline Failing to take the pedometer & 0 & 5 & 0 & - \\
\hline Failing to reset the pedometer & 0 & 5 & 0 & - \\
\hline Forgetting to take the travel log form & 0 & 5 & 0 & - \\
\hline Forgetting to record some trip information & 0 & 5 & 0 & $\begin{array}{l}\text { (One of the subjects failed to record } \\
\text { information on travel log, but failed to } \\
\text { mention this in the questionnaire) }\end{array}$ \\
\hline Failing to record an entire trip on the travel log & 0 & 5 & 0 & - \\
\hline Uncomfortable/ bothersome to wear pedometer & 1 & 4 & 0 & $\begin{array}{l}\text {-Subject thought that the pedometer } \\
\text { would fall off if walked fast }\end{array}$ \\
\hline $\begin{array}{l}\text { Bothersome to carry and record information on } \\
\text { travel log form }\end{array}$ & 3 & 2 & 0 & $\begin{array}{l}\text {-Only when running for class } \\
\text {-Have to carry travel log in hand else } \\
\text { would forget } \\
\text {-Problem when carrying other stuff in } \\
\text { hand }\end{array}$ \\
\hline Difficulty in reading pedometer display screen & 0 & 5 & 0 & - \\
\hline Pedometer unit receiver ever slip off waist & & $\begin{array}{l}\mathrm{AR} \\
\mathrm{CC}\end{array}$ & & - \\
\hline Pedometer affected physical/personal appearance & 0 & 5 & 0 & -Is hardly visible \\
\hline $\begin{array}{l}\text { Pedometer unit interfere with movements or } \\
\text { activities }\end{array}$ & 0 & 5 & 0 & - \\
\hline $\begin{array}{l}\text { Bothered that someone knows about all of the } \\
\text { walking trips you made }\end{array}$ & 0 & 5 & 0 & - \\
\hline
\end{tabular}

Even though the display screen on the pedometer was small, none of the subjects (all college-age males) had problems reading it. One subject thought the pedometer slipped occasionally, but also suggested that the reason maybe due to the subject's loose clothing. The reason could also be due to improper attachment of the device. None of the subjects thought that the pedometer affected their physical/personal appearance or interfered with their daily activities. This result may be biased since most subjects were 
college-age males; the result may have been different if more female subjects were included in the sample. None of the subjects in this experiment reported that they were concerned about someone having information about the trips they made. It is not clear that this question is meaningful here since all trips were self-reported.

It should be noted that these results have been obtained from a small, relatively homogenous sample of participants, i.e., mostly college-age males. Although the focus of this effort was to evaluate the process rather than the results, it is recognized that there is bias in the sample even from a process standpoint. For example, different results could be expected if more females were included in the experiment. College-age males would be expected to have different concerns, levels of technological literacy and travel patterns than other population groups. So in this regard, the findings are biased. Thus, additional research using a larger sample size representing a broader cross section of the population may be needed in order to assess the utility of the pedometer as device to collect pedestrian travel data.

From the experimental results presented here, it is concluded that the pedometer, even though light, unobstrusive and inexpensive, is not acceptable as a data collection device for pedestrian movement information. A primary reason is the absence of any data recording capability of the pedometer. The only data obtained is the step count which must be recorded manually along with origin/destination and clock time information. Thus, the results are susceptible to omissions, errors and manipulation. Furthermore, even if the step count is correct, only a rough estimate of distances and speeds can be obtained. Another disadvantage of using a pedometer is that it does not provide the researcher with information such as delays enroute or path of travel. Thus, 
while pedometers have value for promoting physical activity, they are not suitable for collecting pedestrian data for transportation planning and engineering purposes.

\subsection{Evaluation of Operational Considerations - Hand-Held GPS Unit}

Following a brief set of instructions, the hand-held GPS unit and the travel log form were given to 5 subjects for periods of 24 hours each. The data collected using the hand-held GPS unit was downloaded to a computer for analysis. Along with the handheld GPS unit, the subjects also returned their completed travel data log form which provided information about their trips. When they returned the hand-held GPS unit and travel log forms, they were asked to complete a brief questionnaire about the experience. The digital data from the five subjects was compiled and analyzed to determine various types of trip information such as elevations, origin and destination, trip duration, travel speeds, delays en route and route selected. The procedure followed for the analysis has been described in Chapter 3.

From the travel log form given to each subject, various trip information such as street address and time at origin, street address and time at destination and mode of travel were obtained for all subjects, for all trips made during the 24-hour period. The trip information provided on the travel log form was compared to the trips shown on the map prepared by plotting data points. Any trip which a subject made during the day but failed to record on the travel log form was identified in this fashion. Similarly identified were situations where the subject recorded a trip on the travel log form but forgot to switch on the hand-held GPS unit. The comparison also showed whether the subject recorded the time and address at origin and destination correctly. A summary of the trip information for each subject, during the course of the experiment is shown in Table 32. The table 
presents information about the number of trips made by each subject, average speed, average duration of trips, average distance walked for all trips and average duration of each trip.

Table 32 - Summary of Walking Trip Information Obtained from Subjects in Hand-Held GPS Unit Experiment

\begin{tabular}{|c|c|c|c|c|}
\hline & $\begin{array}{c}\text { No. of Walking } \\
\text { Trips }\end{array}$ & $\begin{array}{c}\text { Average } \\
\text { Speed (fps) }\end{array}$ & $\begin{array}{c}\text { Average Duration } \\
\text { of Each Trip } \\
\text { (min) }\end{array}$ & $\begin{array}{c}\text { Average Distance } \\
\text { Walked per Trip } \\
\text { (miles) }\end{array}$ \\
\hline Subject & 6 & & $9.5 \mathrm{~min}$ & 0.43 \\
\hline 1 & & 4.0 & & 0.35 \\
\hline 2 & 5 & 4.7 & $6.5 \mathrm{~min}$ & 0.50 \\
\hline 3 & 6 & 4.5 & $9.8 \mathrm{~min}$ & 0.37 \\
\hline 4 & 7 & 4.3 & $7.6 \mathrm{~min}$ & \\
\hline & & & & 0.20 \\
\hline 5 & 6 & 3.9 & $4.4 \mathrm{~min}$ & \\
\hline & & & & $\mathbf{0 . 3 7}$ \\
\hline Grand Average & $\mathbf{6}$ & $\mathbf{4 . 3}$ & $\mathbf{7 . 5} \mathbf{m i n}$ & \\
\hline
\end{tabular}

From Table 32, it can be seen that the number of walking trips made by the subjects during the 24-hour duration varied from 5 to 7 trips. The average walking speed ranged from a minimum of 3.9 fps for Subject 5 to a maximum of 4.7 fps for Subject 2, whereas the average duration of each trip varied from around 4 minutes to almost 10 minutes. The average distance walked by each of the subject for their trips ranged from 0.2 miles to 0.5 miles. The grand averages considering all the subjects showed that an average of 6 trips were made at an average speed of $4.3 \mathrm{fps}$ at an average trip duration of 7.5 minutes to cover distance of 0.37 miles.

Considering Subject 1, the trips recorded on the travel log form were compared with the data points obtained from the GPS unit. A typical travel log form with the data recorded by Subject 1 is shown in Table 33. In an ideal situation, the travel log would have been presented verbatim, but in order to insure privacy of the subjects, and to give a 
better description of the origins and destinations, the travel log presented here is the version edited by the researcher.

Table 33 - Typical Data Recorded by Subject 1in the Travel Log Form in Hand-Held GPS Experiment

\begin{tabular}{|c|c|}
\hline $\begin{array}{l}\text { Trip } 1 \\
\end{array}$ & Trip 6 \\
\hline Origin- XXX McCullough Street (Home) & Origin- Pierpont Apts \\
\hline Origin time $-8: 42$ a.m. & Origin time - 10:01 p.m. \\
\hline Mode- Walk & Mode- Bus \\
\hline Destination- Bus-Stop opp. Exxon Univ. Ave. & Destination- Bus-Stop, Willowdale Dr. \\
\hline Destination time- 9:03 a.m. & Destination time- 10:07 p.m. \\
\hline $\begin{array}{r}\text { Trip } 2 \\
\end{array}$ & $\begin{array}{ll}\text { Trip } 7 \\
\end{array}$ \\
\hline Origin- MRB & Origin- Bus-Stop, Willowdale Dr. \\
\hline Origin time - 2:26 p.m. & Origin time - 10:07 p.m. \\
\hline Mode- Walk & Mode- Walk \\
\hline Destination- Taco Bell, Patteson Dr. & Destination- XXX McCullough Street \\
\hline Destination time- $2: 38$ p.m. & Destination time- 10:12 p.m. \\
\hline \multicolumn{2}{|l|}{ Trip 3} \\
\hline Origin- Taco Bell, Patteson Dr. & Origin- \\
\hline Origin time $-2: 59$ p.m. & Origin time - \\
\hline Mode- Walk & Mode- \\
\hline Destination- MRB & Destination- \\
\hline Destination time- 3:09 p.m. & Destination time \\
\hline \multicolumn{2}{|l|}{$\begin{array}{r}\text { Trip } 4 \\
\end{array}$} \\
\hline Origin- MRB & Origin- \\
\hline Origin time - 9:40 p.m. & Origin time - \\
\hline Mode- Walk & Mode- \\
\hline Destination- 7-11 Store & Destination- \\
\hline Destination time- 9:51 p.m. & Destination time \\
\hline \multicolumn{2}{|l|}{$\begin{array}{ll}\text { Trip } 5 \\
\end{array}$} \\
\hline Origin- 7-11 Store & Origin- \\
\hline Origin time - 9:55 p.m. & Origin time - \\
\hline Mode- Walk & Mode- \\
\hline Destination- Pierpont Apts & Destination- \\
\hline Destination time- 9:57 p.m. & Destination time- \\
\hline
\end{tabular}

As observed from the travel log, there were 6 walking trips made by the subject in the 24-hour duration. Although the subjects were asked to record information for only the walking trips made during the duration, the trip log above also shows a bus trip (trip 6) made in between the walking trips. Although the subject recorded one bus trip, he forgot to record the other previous bus trip made between trip 1 and trip 2 (known after trip data was reviewed soon after the experiment, with the subject). This analysis of the travel information recorded by the subject, shows limitation of trip logs. The trips recorded on the travel log form were compared with the data points obtained from the 
GPS unit for each subject. It was observed that the times recorded on travel log form for the individual trips matched very well with the GPS data. There was a variation of only one to two minutes for some of the entries. When comparing the information on origins/destinations from the travel $\log$ to the data points plotted on a map of Morgantown, it was observed that the addresses provided for identification matched closely with the traveled routes.

Two different trips were considered for detailed analysis and presentation. Since the purpose of the study was to assess device capability, trips were selected which were longer than others, included street crossings and varied topography, and were made at different times of the day. Once the two trips were selected, the records for these trips were analyzed to obtain travel data. Travel data refers to information about a trip, including trip time, speeds, delays en route, origins/destinations, route selected, trip length and trip purpose.

The first trip was made by Subject 1 . The data points for this trip were plotted on a street map of Morgantown and are shown in Figure 21. The points shown on the map represent each record made by the GPS device during the course of the trip. The map used was mainly a campus map (obtained from the West Virginia University's GIS Technology Center), which showed the various campuses of West Virginia University in Morgantown and details of streets in the vicinity of the campuses. The process for plotting the data points on a map was described in Chapter 3. Besides a street map, users can also use contour maps, USGS topographic maps and aerial photographs to plot points. 


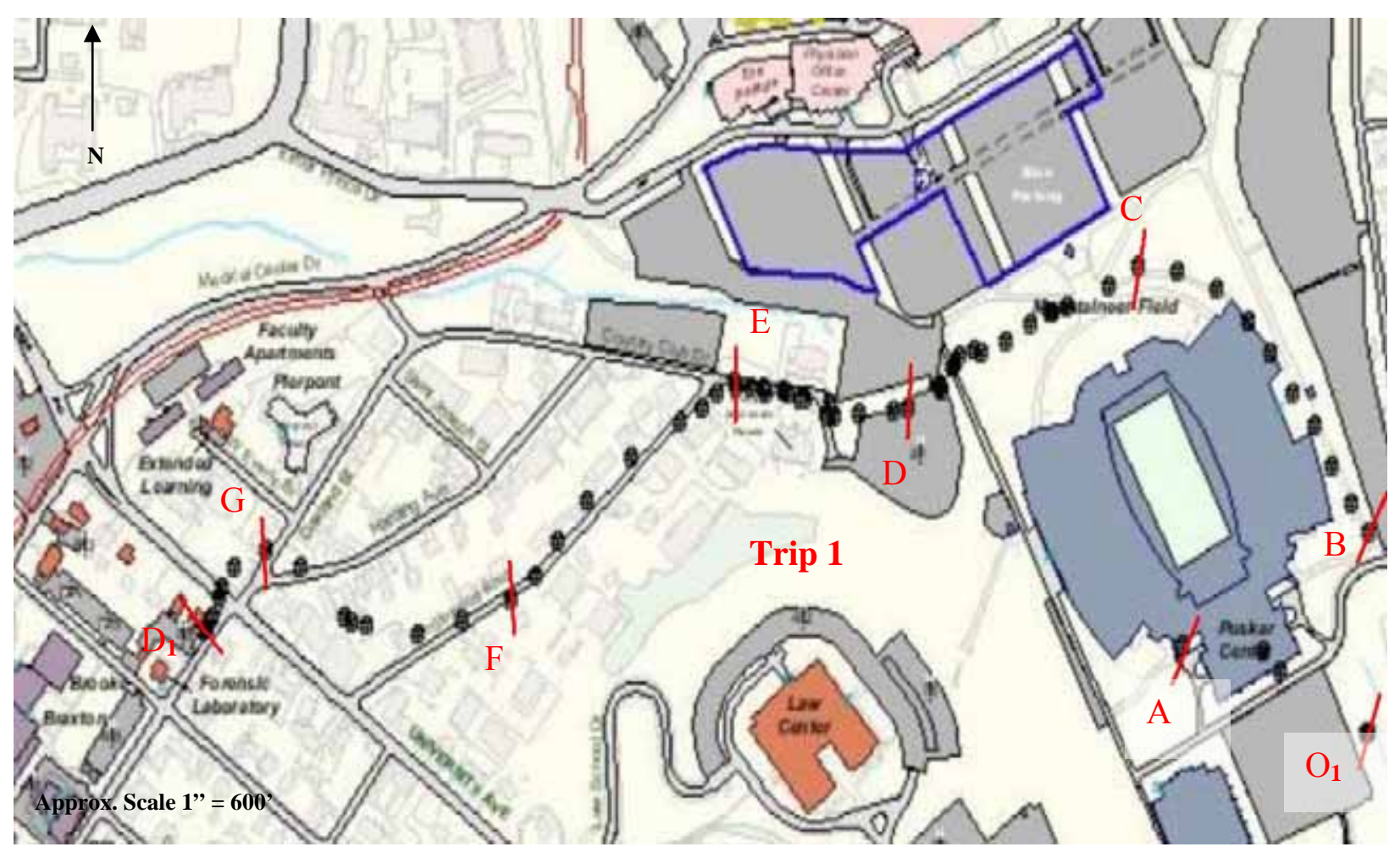

Figure 21: GPS Data for First Trip Analyzed Obtained Using Hand-Held GPS Device, Plotted on Arcview GIS Software

The usable portion of data for Trip 1, which started in the morning, began at a sidewalk along Willowdale Road (point B) located beside the WVU football stadium and ended at a bus-stop on Oakland Street next to the WVU Forensic Laboratory (Point $\mathrm{D}_{1}$ ). Here, points $\mathrm{O}_{1}$ refers to a location where the subject switched on the GPS device and started walking and point $\mathrm{A}$ refers to a location along the route recorded inaccurately by the GPS unit due to lack of satellite signal from satellites, thus rendering both points unusable for analysis. The route along this trip is partially downhill and mostly level with little variation in elevation. The trip was covered partially on grass (next to the stadium), a small distance through a parking lot and partially on sidewalks (latter part). The trip shown in Figure 21 has been divided into smaller sections of unequal lengths and designated by alphabetical characters. These alphabetic characters have been used to denote the points at which the direction of travel seemed to be changing. 
From Figure 21, many types of travel information such as origin/destination, elevations (and, consequently, gradients), trip duration, delays and route selection can be obtained. The plot shown in Figure 21 suggests that Subject 1 did not wait for the initial start-up two minutes, required for the GPS device to configure communication with satellites. This was confirmed by going to the shown location and analyzing the possible route, since reaching point $\mathrm{A}$ on foot from $\mathrm{O}_{1}$ is not feasible. The route selected by the subject was later also confirmed with the subject. This indicates that the GPS device did not establish communication with the satellites until point B on the same route, which was due to the cold start phenomenon mentioned in Chapter 3.

For the trip analyzed (Trip 1), the plot shown in Figure 22 was prepared to create a profile of the trip. This profile was obtained by plotting elevation versus time during the course of trip.

From Figure 22, it can be observed that the data indicate that the elevation drops almost 200 feet in Section AB. As mentioned before, it was observed that the subject apparently did not wait one or two minutes at the start of the trip for the GPS to configure satellites, and thus the elevations recorded until point $\mathrm{B}$ are inaccurate. This was confirmed on-site and with the subject. The significant elevation change shown in Figure 22 does not reflect the actual situation and is likely due to the same cold start phenomenon. Beyond point $\mathrm{B}$, there is little variation in the elevation, with the profile going down from point $\mathrm{B}$ to point $\mathrm{D}$, then moving up between points $\mathrm{D}$ and $\mathrm{F}$, and then again going down between $\mathrm{F}$ and $\mathrm{G}$ before becoming almost level until the end of trip. The subject crossed University Avenue between the points $G$ and destination $D_{1}$. The 
maximum difference in elevation ( 75 feet) was between point $B$ and $D_{1}$. The GPS profile seems to represent the actual topography.

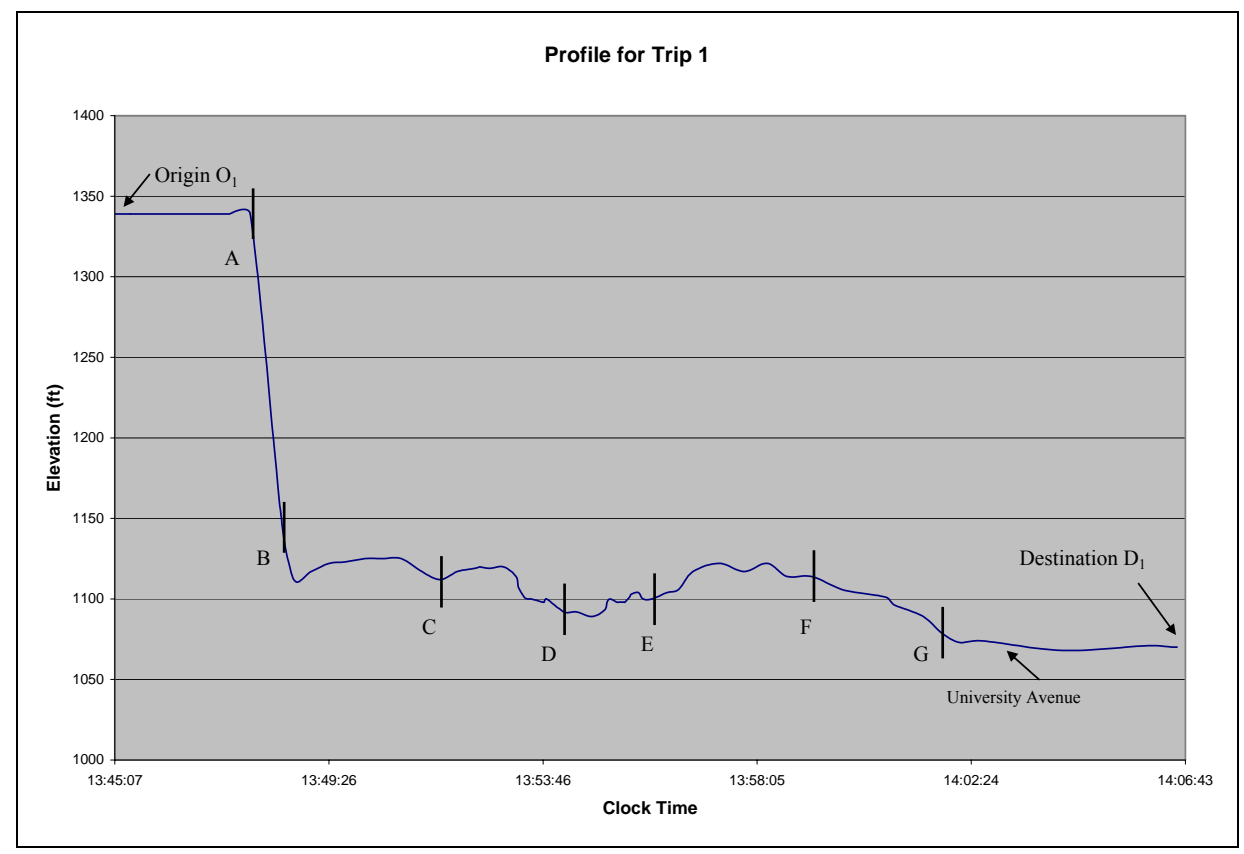

Figure 22: Plot of Elevation Versus Time for Trip 1, Obtained from Hand-Held GPS Unit

Figure 23 shows the speed profile obtained by plotting average speed against distance for various sections making up the trip. The speed profile shows that the average speed of the subject during most of the trip varied from 4 fps to 5 fps. This is within the range of expected walking speeds for healthy, college-age individuals. The average speed between points $\mathrm{C}$ and $\mathrm{D}$ was $6.4 \mathrm{fps}$, which is considered higher than typical average walking speed. Either the subject ran or there was an error in the data associated with the hand-held GPS unit during that section. This increase in the walking speed during the trip suggests a loss of signal, but this was not evident from the data obtained. One of the possibilities may be due to the reduction in the number of satellites communicating with satellites which could have been an error in data recording due to the hand-held GPS unit not being held upright. The plotted points in Figure 21 do not 
show the stretch between points $\mathrm{C}$ and $\mathrm{D}$ to be in the vicinity of tall buildings and thus loss of signal is not likely due to tall buildings.

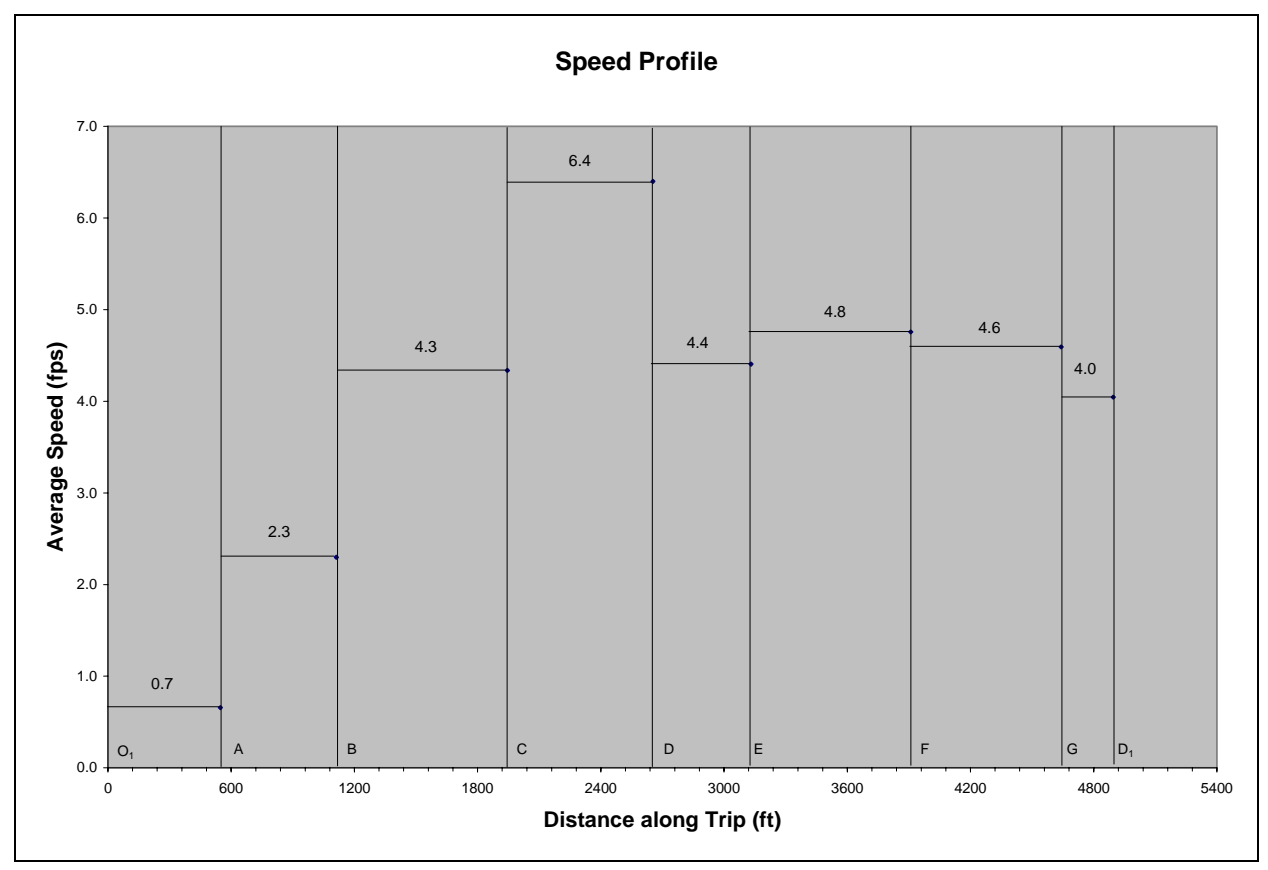

Figure 23: Speed Profile for Trip 1 Obtained from Hand-Held GPS Device Experiment

Table 34 shows the downloaded data for the trip. A new trip is designated with a 'True' message in the New_Seg column (i.e. new segment). A new trip starts when there is a loss of signal or the device has been turned on for a new trip. From the table, it can be observed that there is no loss of signal during the course of the trip. The 'True' message in the initial part of the trip log was because the GPS unit was trying to connect/communicate with the satellites. As mentioned before, there seems to be a small loss of signal between points $\mathrm{C}$ and $\mathrm{D}$, but this is not evident from the table of data obtained. The duration of trip recorded on the trip log was 21 minutes; the GPS data also indicated a trip duration of 21 minutes (Time at $\mathrm{D}_{1}-$ Time at $\mathrm{O}_{1}=14: 06-13: 45=21$ minutes). The trip origin and destination seen from the plotted data points was consistent with the data recorded on the travel log form. 
Table 34 - Sample Data Obtained from Hand-Held GPS Unit for Trip 1

\begin{tabular}{|c|c|c|c|c|c|c|c|c|c|c|}
\hline Type & Ident & Lat & Long & y_proj & x proj & New_seg & Altitude & $\begin{array}{c}\text { Date and Time } \\
\text { (GMT) }\end{array}$ & $\mathrm{m} / \mathrm{s}$ & \\
\hline TRACK & ACTIVE LOG & 39.64860678 & -79.9532 & 418677.9489 & 1840896.939 & TRUE & 1339 & 2005/02/21-13:45:07 & 0.2 & $\mathrm{O}_{1}$ \\
\hline TRACK & ACTIVE LOG & 39.64860678 & -79.9532 & 418677.9794 & 1840890.896 & FALSE & 1339 & $2005 / 02 / 21-13: 45: 26$ & 0.2 & \\
\hline TRACK & ACTIVE LOG & 39.64897156 & -79.954 & 418812.0358 & 1840655.933 & TRUE & 1339 & 2005/02/21-13:47:10 & 0.3 & \\
\hline TRACK & ACTIVE LOG & 39.6491003 & -79.9542 & 418859.1721 & 1840607.835 & FALSE & 1339 & $2005 / 02 / 21-13: 47: 26$ & 0.1 & \\
\hline TRACK & ACTIVE LOG & 39.64914322 & -79.955 & 418875.9059 & 1840390.406 & FALSE & 1339 & $2005 / 02 / 21-13: 47: 51$ & 0.2 & A \\
\hline TRACK & ACTIVE LOG & 39.64985132 & -79.9532 & 419131.2879 & 1840893.183 & TRUE & 1145 & $2005 / 02 / 21-13: 48: 30$ & 1.2 & B \\
\hline TRACK & ACTIVE LOG & 39.65004444 & -79.9533 & 419201.8429 & 1840851.246 & FALSE & 1112 & 2005/02/21-13:48:44 & 1.1 & \\
\hline TRACK & ACTIVE LOG & 39.65028048 & -79.9535 & 419288.0921 & 1840797.304 & FALSE & 1117 & $2005 / 02 / 21-13: 49: 05$ & 1.2 & \\
\hline TRACK & ACTIVE LOG & 39.65053797 & -79.9537 & 419382.1238 & 1840749.444 & FALSE & 1122 & $2005 / 02 / 21-13: 49: 27$ & 1.5 & \\
\hline TRACK & ACTIVE LOG & 39.65073109 & -79.9539 & 419452.7706 & 1840689.381 & FALSE & 1123 & 2005/02/21-13:49:46 & 1.5 & \\
\hline TRACK & ACTIVE LOG & 39.65098858 & -79.9541 & 419546.8636 & 1840629.437 & FALSE & 1125 & 2005/02/21-13:50:10 & 1.4 & \\
\hline TRACK & ACTIVE LOG & 39.6511817 & -79.9544 & 419617.5107 & 1840569.378 & FALSE & 1125 & $2005 / 02 / 21-13: 50: 31$ & 1.4 & \\
\hline TRACK & ACTIVE LOG & 39.65137482 & -79.9547 & 419688.2803 & 1840485.149 & FALSE & 1125 & 2005/02/21-13:50:54 & 1.4 & \\
\hline TRACK & ACTIVE LOG & 39.65148211 & -79.955 & 419727.91 & 1840376.597 & FALSE & 1117 & 2005/02/21-13:51:19 & 1.2 & \\
\hline TRACK & ACTIVE LOG & 39.65152502 & -79.9554 & 419744.0599 & 1840273.968 & FALSE & 1112 & 2005/02/21-13:51:41 & 1.3 & C \\
\hline TRACK & ACTIVE LOG & 39.65139627 & -79.9557 & 419697.5932 & 1840189.146 & FALSE & 1117 & 2005/02/21-13:52:02 & 1 & \\
\hline TRACK & ACTIVE LOG & 39.65126753 & -79.9561 & 419651.2531 & 1840080.16 & FALSE & 1119 & $2005 / 02 / 21-13: 52: 23$ & 1.2 & \\
\hline TRACK & ACTIVE LOG & 39.65122461 & -79.9562 & 419635.8347 & 1840037.787 & FALSE & 1120 & $2005 / 02 / 21-13: 52: 29$ & 1.2 & \\
\hline TRACK & ACTIVE LOG & 39.65122461 & -79.9563 & 419635.8654 & 1840031.745 & FALSE & 1120 & 2005/02/21-13:52:30 & 1.2 & \\
\hline TRACK & ACTIVE LOG & 39.65116024 & -79.9565 & 419612.6956 & 1839977.253 & FALSE & 1119 & 2005/02/21-13:52:41 & 1.3 & \\
\hline TRACK & ACTIVE LOG & 39.65105295 & -79.9567 & 419573.9543 & 1839910.594 & FALSE & 1120 & $2005 / 02 / 21-13: 52: 58$ & 1.5 & \\
\hline TRACK & ACTIVE LOG & 39.65096712 & -79.9569 & 419543.0297 & 1839843.978 & FALSE & 1114 & $2005 / 02 / 21-13: 53: 13$ & 1.5 & \\
\hline TRACK & ACTIVE LOG & 39.65098858 & -79.957 & 419550.9384 & 1839825.89 & FALSE & 1107 & 2005/02/21-13:53:16 & 1.6 & \\
\hline TRACK & ACTIVE LOG & 39.65094566 & -79.9571 & 419535.4898 & 1839789.562 & FALSE & 1101 & $2005 / 02 / 21-13: 53: 23$ & 1.5 & \\
\hline TRACK & ACTIVE LOG & 39.65090275 & -79.9572 & 419519.9218 & 1839777.398 & FALSE & 1100 & $2005 / 02 / 21-13: 53: 27$ & 1.5 & \\
\hline TRACK & ACTIVE LOG & 39.65088129 & -79.9572 & 419512.1668 & 1839765.276 & FALSE & 1100 & 2005/02/21-13:53:31 & 3.5 & \\
\hline TRACK & ACTIVE LOG & 39.65073109 & -79.9573 & 419457.5812 & 1839740.831 & FALSE & 1098 & $2005 / 02 / 21-13: 53: 45$ & 4.5 & \\
\hline TRACK & ACTIVE LOG & 39.65075254 & -79.9573 & 419465.4249 & 1839734.829 & FALSE & 1098 & 2005/02/21-13:53:47 & 4.8 & \\
\hline TRACK & ACTIVE LOG & 39.650774 & -79.9573 & 419473.2414 & 1839734.868 & FALSE & 1100 & 2005/02/21-13:53:50 & 1.5 & \\
\hline TRACK & ACTIVE LOG & 39.6506238 & -79.9576 & 419418.9636 & 1839650.005 & FALSE & 1092 & 2005/02/21-13:54:11 & 1.6 & D \\
\hline TRACK & ACTIVE LOG & 39.65060234 & -79.9578 & 419411.3626 & 1839607.674 & FALSE & 1092 & $2005 / 02 / 21-13: 54: 26$ & 1.4 & \\
\hline TRACK & ACTIVE LOG & 39.65058088 & -79.9581 & 419404.008 & 1839517.007 & FALSE & 1089 & $2005 / 02 / 21-13: 54: 45$ & 0.5 & \\
\hline TRACK & ACTIVE LOG & 39.65055943 & -79.9583 & 419396.534 & 1839450.509 & FALSE & 1093 & $2005 / 02 / 21-13: 55: 00$ & 1.4 & \\
\hline TRACK & ACTIVE LOG & 39.65055943 & -79.9584 & 419396.6265 & 1839432.382 & FALSE & 1098 & 2005/02/21-13:55:03 & 1.8 & \\
\hline TRACK & ACTIVE LOG & 39.65064526 & -79.9584 & 419427.8891 & 1839432.541 & FALSE & 1100 & $2005 / 02 / 21-13: 55: 07$ & 1.4 & \\
\hline TRACK & ACTIVE LOG & 39.65066671 & -79.9586 & 419436.0412 & 1839366.123 & FALSE & 1098 & 2005/02/21-13:55:15 & 1.4 & \\
\hline TRACK & ACTIVE LOG & 39.65066671 & -79.9587 & 419436.1028 & 1839354.038 & FALSE & 1098 & 2005/02/21-13:55:18 & 1.3 & \\
\hline TRACK & ACTIVE LOG & 39.65070963 & -79.9588 & 419451.8902 & 1839323.912 & FALSE & 1098 & $2005 / 02 / 21-13: 55: 23$ & 1.5 & \\
\hline TRACK & ACTIVE LOG & 39.65073109 & -79.9588 & 419459.7684 & 1839311.867 & FALSE & 1098 & $2005 / 02 / 21-13: 55: 25$ & 1.4 & \\
\hline TRACK & ACTIVE LOG & 39.65070963 & -79.959 & 419452.1988 & 1839263.494 & FALSE & 1101 & $2005 / 02 / 21-13: 55: 30$ & 1.2 & \\
\hline TRACK & ACTIVE LOG & 39.65075254 & -79.959 & 419467.8591 & 1839257.531 & FALSE & 1103 & $2005 / 02 / 21-13: 55: 33$ & 1.3 & \\
\hline TRACK & ACTIVE LOG & 39.65075254 & -79.9591 & 419468.0443 & 1839221.283 & FALSE & 1104 & 2005/02/21-13:55:41 & 1.2 & \\
\hline TRACK & ACTIVE LOG & 39.65075254 & -79.9593 & 419468.2296 & 1839185.031 & FALSE & 1100 & $2005 / 02 / 21-13: 55: 47$ & 1.4 & \\
\hline TRACK & ACTIVE LOG & 39.650774 & -79.9593 & 419476.0771 & 1839179.029 & FALSE & 1100 & 2005/02/21-13:55:57 & 1.5 & $E$ \\
\hline TRACK & ACTIVE LOG & 39.65070963 & -79.9595 & 419452.8782 & 1839130.575 & FALSE & 1104 & $2005 / 02 / 21-13: 56: 16$ & 1.2 & \\
\hline TRACK & ACTIVE LOG & 39.6506238 & -79.9596 & 419421.832 & 1839088.124 & FALSE & 1106 & 2005/02/21-13:56:30 & 1.5 & \\
\hline TRACK & ACTIVE LOG & 39.65051651 & -79.9598 & 419383.0312 & 1839033.548 & FALSE & 1117 & $2005 / 02 / 21-13: 56: 47$ & 1.4 & \\
\hline TRACK & ACTIVE LOG & 39.65030193 & -79.9603 & 419305.5537 & 1838900.229 & FALSE & 1122 & $2005 / 02 / 21-13: 57: 19$ & 1.8 & \\
\hline TRACK & ACTIVE LOG & 39.65002298 & -79.9607 & 419204.569 & 1838778.874 & FALSE & 1117 & 2005/02/21-13:57:49 & 1.4 & \\
\hline TRACK & ACTIVE LOG & 39.64976549 & -79.961 & 419111.1843 & 1838699.849 & FALSE & 1122 & 2005/02/21-13:58:17 & 1.6 & \\
\hline TRACK & ACTIVE LOG & 39.64955091 & -79.9612 & 419033.3363 & 1838639.029 & FALSE & 1114 & $2005 / 02 / 21-13: 58: 41$ & 1.2 & \\
\hline TRACK & ACTIVE LOG & 39.64940071 & -79.9614 & 418978.9691 & 1838572.289 & FALSE & 1114 & 2005/02/21-13:59:11 & 1.5 & $\mathbf{F}$ \\
\hline TRACK & ACTIVE LOG & 39.64927197 & -79.9619 & 418932.7602 & 1838439.127 & FALSE & 1106 & 2005/02/21-13:59:47 & 1.5 & \\
\hline TRACK & ACTIVE LOG & 39.64918613 & -79.9623 & 418902.1157 & 1838318.128 & FALSE & 1103 & $2005 / 02 / 21-14: 00: 18$ & 1.9 & \\
\hline TRACK & ACTIVE LOG & 39.64922905 & -79.9628 & 418918.4643 & 1838179.247 & FALSE & 1101 & $2005 / 02 / 21-14: 00: 41$ & 1.2 & \\
\hline TRACK & ACTIVE LOG & 39.64925051 & -79.963 & 418926.4676 & 1838143.035 & FALSE & 1098 & $2005 / 02 / 21-14: 00: 47$ & 1.2 & \\
\hline TRACK & ACTIVE LOG & 39.64929342 & -79.963 & 418942.1904 & 1838124.991 & FALSE & 1096 & $2005 / 02 / 21-14: 00: 52$ & 1.2 & \\
\hline TRACK & ACTIVE LOG & 39.64959383 & -79.9635 & 419052.2342 & 1838004.717 & FALSE & 1089 & $2005 / 02 / 21-14: 01: 26$ & 1.3 & \\
\hline TRACK & ACTIVE LOG & 39.64970112 & -79.9638 & 419091.8121 & 1837908.25 & FALSE & 1079 & 2005/02/21-14:01:48 & 1.4 & G \\
\hline TRACK & ACTIVE LOG & 39.64959383 & -79.9641 & 419053.1699 & 1837823.462 & FALSE & 1073 & $2005 / 02 / 21-14: 02: 10$ & 1.4 & \\
\hline TRACK & ACTIVE LOG & 39.64946508 & -79.9642 & 419006.4304 & 1837793.013 & FALSE & 1074 & $2005 / 02 / 21-14: 02: 37$ & 1.5 & \\
\hline TRACK & ACTIVE LOG & 39.64942217 & -79.9642 & 418990.8634 & 1837780.847 & FALSE & 1068 & $2005 / 02 / 21-14: 04: 15$ & 1.2 & \\
\hline TRACK & ACTIVE LOG & 39.64929342 & -79.9643 & 418944.0615 & 1837762.48 & FALSE & 1071 & $2005 / 02 / 21-14: 05: 58$ & 1.1 & \\
\hline TRACK & ACTIVE LOG & 39.64920759 & -79.9644 & 418912.8614 & 1837750.234 & FALSE & 1070 & $2005 / 02 / 21-14: 06: 27$ & 0.8 & \\
\hline TRACK & ACTIVE LOG & 39.64918613 & -79.9644 & 418905.0761 & 1837744.151 & FALSE & 1070 & $2005 / 02 / 21-14: 06: 33$ & 0.2 & $D_{1}$ \\
\hline
\end{tabular}

A 'Delay' is considered when the speed of the device user becomes zero for a

small duration of time during a trip. The $\mathrm{m} / \mathrm{s}$ column in table does not show any instance

when the speed was zero. From the data shown in Table 34, it can also be observed that

the data points were recorded at 15 to 30 second intervals, which means that delays of

less than 15 seconds will not be recorded and, thus, there will not be any accounting for 
delays of short durations. The approximate length of the trip was calculated from the plotted data in GIS software, by measuring the length of various stretches from $\mathrm{O}_{1}$ to $\mathrm{D}_{1}$ (using the distance measuring tool) and found to be 0.92 mile.

Figure 24 shows the plotted data for the second trip analyzed (made by Subject 3) for this experiment. Trip 2 started at the University's Medical Center housing on Van Voorhis Road $\left(\mathrm{O}_{2}\right)$ and ended at a residential building on University Avenue $\left(\mathrm{D}_{2}\right)$ and took place late at night. Figure 24 shows the plotted data-points of the trip on a map of the campus. The route along this trip is generally level during the initial half of the trip and then uphill for the latter half. The trip shown in Figure 24 has been divided into smaller sections of unequal lengths; the starting and ending points of each section are denoted by alphabetic characters.

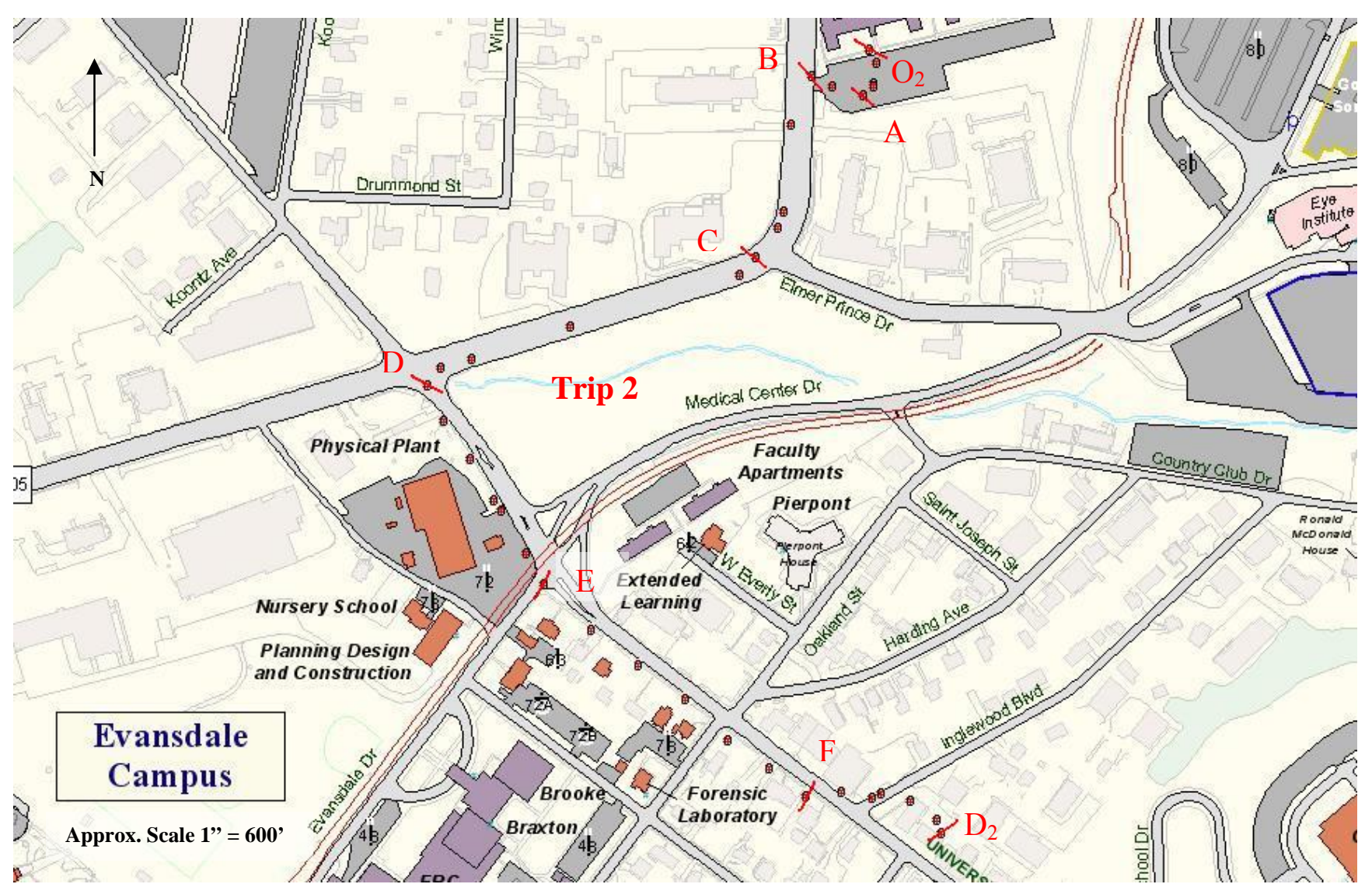

Figure 24: GPS Data for Second Trip Analyzed for Subject 3 Obtained Using Hand-Held GPS Device, Plotted on Arcview GIS Software. 
From Figure 24, it can be noted that sections BC and CD (from the points plotted) indicate that the subject was in the road, which is not true. It was confirmed later with the subject that the route selected for the trip was the sidewalk on the east side of Van Voorhis Road (a 5-lane arterial roadway). The subject used the sidewalk on the south side of Patteson Drive and the south west side of University Avenue. This indicates that, for some portion of the trip, the data recorded by the GPS is not accurate which acts as a limitation for some of its applications.

Although the recorded data is not precise with respect to the physical location of the user, it gives a fairly good idea of the speeds, route selection and origins and destinations.

Table 35 presents the data collected by the hand-held GPS unit for the second trip. The duration of trip recorded in the trip $\log$ was 15 minutes whereas the GPS data showed trip duration of $12 \mathrm{~min} 50 \mathrm{sec}$. The trip origin and destination seen from the plotted data points matched with the data recorded on the travel log form.

As mentioned before, delay is considered when the speed of the device user becomes zero for a short period of time during a trip. The table does not show any instance when the speed was zero. From the data shown in Table 35, it can be observed that almost all data points were recorded at 15 to 45 second intervals. This means that delays of less than 15 seconds will not be recorded. A delay was expected when the subject crossed University Avenue, which carries a considerable amount of traffic. However, since the trip was carried out late at night, it appears that the subject crossed the street without having to stop to find a gap in traffic. The approximate length of the trip was calculated from the plotted data and found to be 0.61 mile. 
Table 35 - Sample Data Obtained from Hand-Held GPS unit for Trip 2

\begin{tabular}{|c|c|c|c|c|c|c|c|c|c|c|}
\hline Type & Ident & Lat & Long & y_proj & x_proj & New_seg & Altitude & $\begin{array}{l}\text { Date and Time } \\
\text { (GMT) }\end{array}$ & $\mathrm{m} / \mathrm{s}$ & \\
\hline TRACK & ACTIVE LOG & 39.65369225 & -79.96293783 & 420544.286 & 1838157.415 & TRUE & 995 & $2005 / 03 / 05-06: 46: 42$ & 0.3 & $\mathrm{O}_{2}$ \\
\hline TRACK & ACTIVE LOG & 39.65360641 & -79.96287346 & 420512.9265 & 1838175.377 & FALSE & 999 & $2005 / 03 / 05-06: 46: 47$ & 0.3 & \\
\hline TRACK & ACTIVE LOG & 39.65347767 & -79.96289492 & 420466.0656 & 1838169.093 & FALSE & 999 & $2005 / 03 / 05-06: 47: 12$ & 0.4 & \\
\hline TRACK & ACTIVE LOG & 39.65345621 & -79.96289492 & 420458.249 & 1838169.053 & FALSE & 1010 & $2005 / 03 / 05-06: 47: 30$ & 1.2 & \\
\hline TRACK & ACTIVE LOG & 39.65339184 & -79.96298075 & 420434.9276 & 1838144.766 & FALSE & 1008 & $2005 / 03 / 05-06: 47: 58$ & 1.3 & A \\
\hline TRACK & ACTIVE LOG & 39.65345621 & -79.96323824 & 420458.7473 & 1838072.39 & FALSE & 1011 & $2005 / 03 / 05-06: 48: 13$ & 1.2 & \\
\hline TRACK & ACTIVE LOG & 39.65352058 & -79.9634099 & 420482.4426 & 1838024.179 & FALSE & 1010 & $2005 / 03 / 05-06: 48: 42$ & 1.5 & B \\
\hline TRACK & ACTIVE LOG & 39.65319872 & -79.96358156 & 420365.4583 & 1837975.243 & FALSE & 1010 & $2005 / 03 / 05-06: 49: 03$ & 1.4 & \\
\hline TRACK & ACTIVE LOG & 39.65264082 & -79.96364594 & 420162.3435 & 1837956.068 & FALSE & 1010 & $2005 / 03 / 05-06: 49: 20$ & 1.4 & \\
\hline TRACK & ACTIVE LOG & 39.65253353 & -79.96368885 & 420123.3268 & 1837943.784 & FALSE & 1010 & $2005 / 03 / 05-06: 49: 57$ & 1.4 & \\
\hline TRACK & ACTIVE LOG & 39.65234041 & -79.96388197 & 420053.2659 & 1837889.047 & FALSE & 1010 & $2005 / 03 / 05-06: 50: 13$ & 1.3 & C \\
\hline TRACK & ACTIVE LOG & 39.65217809 & $\begin{array}{l}-79.96394018 \\
\end{array}$ & 419952.2879 & 1837671.083 & FALSE & 1010 & 2005/03/05-06:50:45 & 1.3 & \\
\hline TRACK & ACTIVE LOG & 39.6518898 & -79.96542692 & 419891.3866 & 1837453.203 & FALSE & 1011 & $2005 / 03 / 05-06: 51: 07$ & 1.5 & \\
\hline TRACK & ACTIVE LOG & 39.65167522 & -79.96626377 & 419814.4503 & 1837217.174 & FALSE & 1013 & $2005 / 03 / 05-06: 51: 22$ & 1.4 & \\
\hline TRACK & ACTIVE LOG & 39.65161085 & -79.96652126 & 419791.3807 & 1837144.553 & FALSE & 1013 & $2005 / 03 / 05-06: 51: 49$ & 1.3 & \\
\hline TRACK & ACTIVE LOG & 39.65150356 & -79.96662855 & 419752.4585 & 1837114.141 & FALSE & 1013 & 2005/03/05-06:52:10 & 1.3 & D \\
\hline TRACK & ACTIVE LOG & 39.65125817 & -79.96650121 & 419671.8531 & 1837158.012 & FALSE & 1012 & $2005 / 03 / 05-06: 52: 33$ & 1.3 & \\
\hline TRACK & ACTIVE LOG & 39.65098212 & -79.96625421 & 419523.9129 & 1837221.917 & FALSE & 1012 & 2005/03/05-06:52:57 & 1.3 & \\
\hline TRACK & ACTIVE LOG & 39.65075254 & -79.96607065 & 419478.0933 & 1837269.805 & FALSE & 1011 & 2005/03/05-06:53:20 & 1.4 & \\
\hline TRACK & ACTIVE LOG & 39.65068817 & -79.96600628 & 419454.5533 & 1837287.808 & FALSE & 1013 & 2005/03/05-06:53:48 & 1.2 & \\
\hline TRACK & ACTIVE LOG & 39.65040922 & $\begin{array}{l}-79.9657917 \\
\end{array}$ & 419352.6359 & 1837347.7 & FALSE & 1013 & $2005 / 03 / 05-06: 54: 26$ & 1.5 & \\
\hline TRACK & ACTIVE LOG & 39.6502161 & -79.9656415 & 419282.0751 & 1837389.626 & FALSE & 1013 & $2005 / 03 / 05-06: 55: 02$ & 1.5 & $E$ \\
\hline TRACK & ACTIVE LOG & 39.64996501 & -79.96525891 & 419190.2835 & 1837142.835 & FALSE & 1013 & $2005 / 03 / 05-06: 55: 27$ & 1.3 & \\
\hline TRACK & ACTIVE LOG & 39.64971586 & $\begin{array}{l}-79.96487902 \\
\end{array}$ & 419090.1918 & 1837481.418 & FALSE & 1010 & 2005/03/05-06:55:39 & 1.3 & \\
\hline TRACK & ACTIVE LOG & 39.64945975 & -79.96449001 & 418996.2175 & 1837915.206 & FALSE & 1010 & 2005/03/05-06:56:04 & 1.3 & \\
\hline TRACK & ACTIVE LOG & 39.64920759 & -79.96409655 & 418912.4868 & 1837822.735 & FALSE & 1010 & $2005 / 03 / 05-06: 56: 37$ & 1.6 & \\
\hline TRACK & ACTIVE LOG & 39.64902572 & -79.96376398 & 418780.8352 & 1837986.831 & FALSE & 1010 & $2005 / 03 / 05-06: 56: 51$ & 1.3 & \\
\hline TRACK & ACTIVE LOG & 39.64884281 & -79.96343136 & 418778.6533 & 1838009.348 & FALSE & 1010 & $2005 / 03 / 05-06: 57: 22$ & 1.5 & $F$ \\
\hline TRACK & ACTIVE LOG & 39.64885217 & -79.96315241 & 418783.7125 & 1838779.128 & FALSE & 1010 & $2005 / 03 / 05-06: 57: 48$ & 1.3 & \\
\hline TRACK & ACTIVE LOG & 39.64884281 & -79.96287346 & 418777.8434 & 1838166.437 & FALSE & 1008 & 2005/03/05-06:58:03 & 1.2 & \\
\hline TRACK & ACTIVE LOG & 39.64886427 & -79.96280909 & 418785.5665 & 1838184.602 & FALSE & 1008 & 2005/03/05-06:58:38 & 1.2 & \\
\hline TRACK & ACTIVE LOG & 39.64882135 & -79.96255159 & 418769.5601 & 1838257.027 & FALSE & 1010 & 2005/03/05-06:59:02 & 1.4 & \\
\hline TRACK & ACTIVE LOG & 39.64869261 & -79.96233702 & 418722.3571 & 1838317.202 & FALSE & 1008 & $2005 / 03 / 05-06: 59: 23$ & 1.4 & \\
\hline TRACK & ACTIVE LOG & 39.64860678 & $\begin{array}{l}-79.9622941 \\
\end{array}$ & 418691.0324 & 1838329.127 & FALSE & 1008 & 2005/03/05-06:59:37 & 1.2 & $D_{2}$ \\
\hline
\end{tabular}

For Trip 2 from $\mathrm{O}_{2}$ to $\mathrm{D}_{2}$, the graph shown in Figure 25 was obtained for the profile of the trip. It can be seen that the profile obtained from the recorded points had several peaks during the trip. The elevations varied from 999 feet to 1013 feet.

From Figure 25, it is noted that the profile indicates a sharp increase in elevation between the origin, $\mathrm{O}_{2}$ and point A. As shown in Figure 24, Point A was located in the parking lot outside WVU Medical Center Apartments.

The elevation difference between origin, $\mathrm{O}_{2}$ (elevation 999 feet) and point $\mathrm{A}$ (elevation 1008 feet) is not 9 feet as recorded by the GPS unit. This discrepancy may be due to the GPS unit trying to connect to satellites in the initial part of the trip. The undulating profile shown in Figure 25 does not reflect actual condition, since this area is relatively level with gradual elevation changes. 


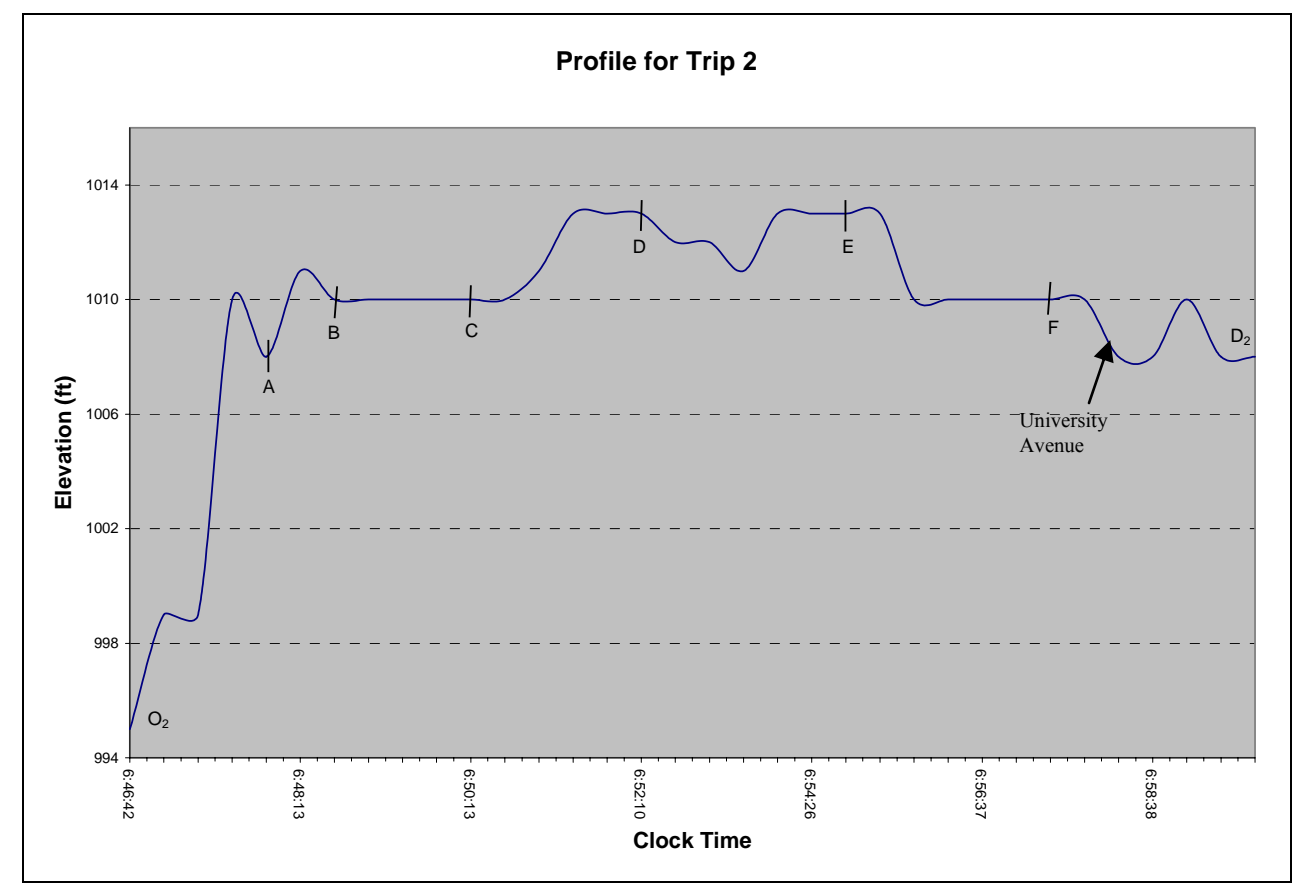

Figure 25: Plot of Elevation Versus Time for Trip 2, Obtained from Hand-Held GPS Unit

As mentioned previously, the route along this trip is rather flat initially, and then goes steadily uphill beyond point E. This is not reflected in Figure 25. In fact, the profile in Figure 25 shows a decrease in elevation from point $\mathrm{E}$ onwards. The subject crossed University Avenue in the section between point $\mathrm{F}$ and destination $\left(\mathrm{D}_{2}\right)$.

Figure 26 shows the variation in average speeds of the subject in the different sections of the trip. The average speed from the origin $\left(\mathrm{O}_{2}\right)$ to point A was low and may be because the subject was waiting for the GPS unit to communicate with the satellites. After Point A, speeds were fairly uniform with average speeds varying from a low of 4.1 fps to $4.7 \mathrm{fps}$. 


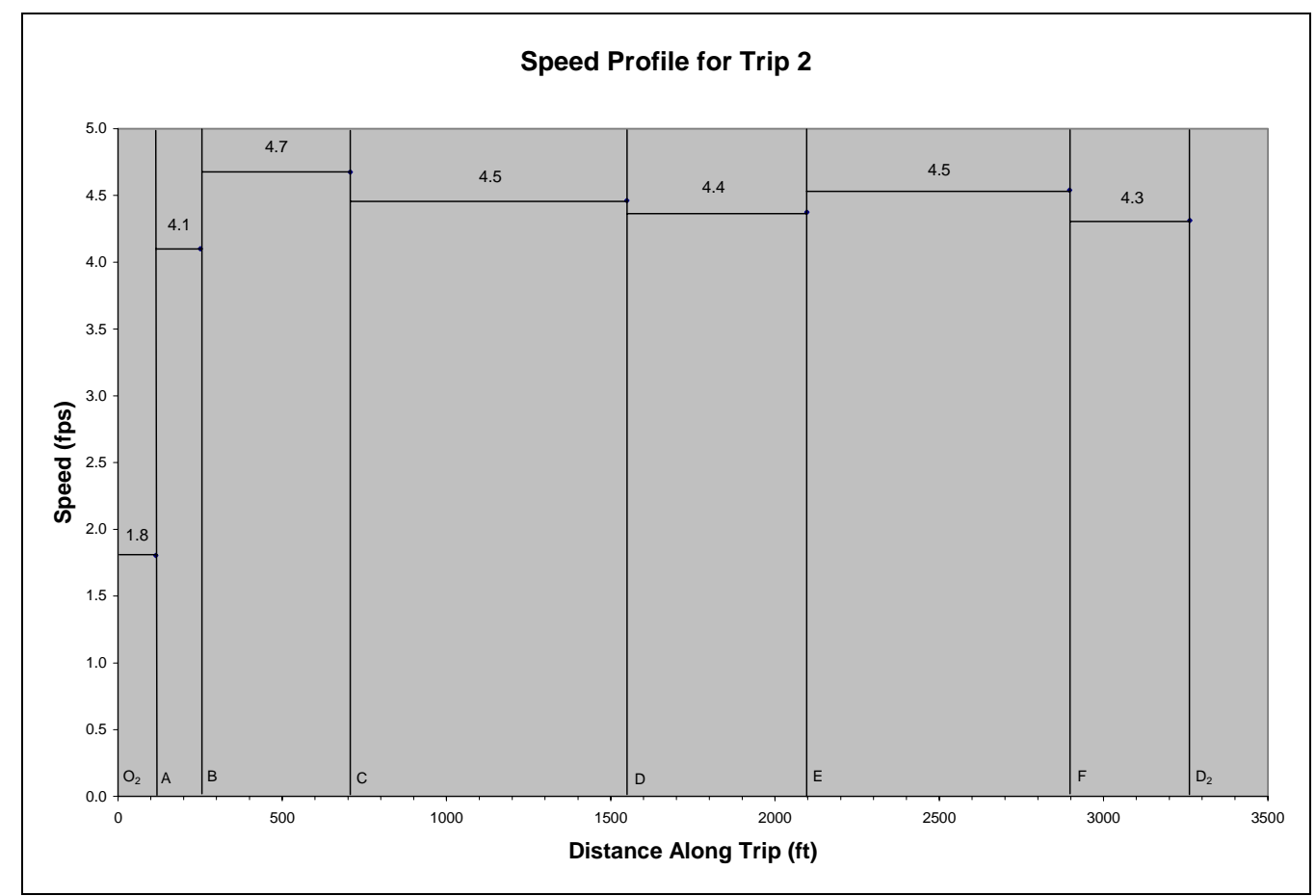

Figure 26: Speed Profile for Trip 2 Obtained from Hand-Held GPS Device Experiment

The subject's highest speed occurred between B and C. This may be attributed to the relatively level gradient and good surface characteristics of the sidewalk in this section. The next highest average speed occurred between points $\mathrm{E}$ and $\mathrm{F}$, which are along University Avenue. This stretch, although uphill has a good-condition sidewalk and thus the subject was able to maintain speed in that section. A narrow buffer exists between the sidewalk and the road in this area, which is not present between B and C. As mentioned earlier, the subject crossed University Avenue between $\mathrm{F}$ and $\mathrm{D}_{2}$. Figure 26 shows only a slight decrease in the travel speed, indicating that the subject did not really slow down to find a gap in traffic and must have crossed easily. This was likely due to the trip being made late at night when there was minimal traffic on the road.

In similar manner, trip information can be obtained for all trips made by each subject. Besides gathering and determining various travel characteristics of individuals, this information can also be used for identifying or improving the existing pedestrian 
facilities on the route used more often by pedestrians. Thus, the information obtained from the GPS data can be used for traffic planning purposes and for designing facilities for pedestrians. Pedestrian connectivity to areas such as shopping centers, banks, public transit and eating places can also be identified and improved upon using the data obtained through the GPS devices.

This experiment involved costs for the hand-held GPS unit, the cost for a computer to which the data would be downloaded and the cost of cable for connecting the computer with the hand-held GPS unit. The hand-held GPS unit used in this study cost approximately $\$ 100$. Although the hand-held unit records less detailed information than the wearable GPS data-logger, it may prove to a better option for collecting data on a large scale as it is about one-tenth the price of the wearable data-logger. In addition, the hand-held GPS unit is smaller and easier to carry in comparison to the wearable unit.

The effort involved in processing the data involves downloading data to a computer and overlaying the data points on a street map of the area. The procedure followed for post-processing of collected data has been explained in detail in Chapter 3 . The post-processing, i.e., downloading, analyzing and overlaying procedure for all trips (8-12 trips) recorded by a subject required about 2 to 2.5 hours for each sample subject. The post-processing also included analyzing the responses to the questionnaire.

The educational requirement for administering this experiment, and consequently data processing, includes a minimum of technician-level education in the field of transportation, basic knowledge of computers and basic understanding of GPS technology and GIS software. A formal training course in GIS and software working on GIS platform will be helpful but not necessary. The person conducting the experiments 
should have good analytical skills to interpret the data collected and should be comfortable with high-school mathematics.

The questionnaires were also analyzed in order to address concerns about user response/acceptability to the device and the experience. A summary of the questionnaire results is presented in Table 36. From Table 36, it can be observed that subjects reported that they followed the correct procedure for data collection, as described in the instructions. Subjects reported that they recorded the trips correctly on the travel log form, without failing to record trips (one exception) and their origins/destinations. This fact was also confirmed from the travel log data.

Table 36-Summary of Subject Responses to Questionnaire on Hand-Held GPS Device

\begin{tabular}{|c|c|c|c|c|}
\hline Question & Yes & No & Blank & Comments \\
\hline Failing to take the hand-held GPS device & 0 & 5 & 0 & - \\
\hline Failing to switch on the hand-held GPS device? & 0 & 5 & 0 & - \\
\hline Forgetting to wait the two minutes during start-up & 2 & 3 & 0 & $\begin{array}{l}\text {-Subject 3, Once } \\
\text {-Subject 4, Twice }\end{array}$ \\
\hline Forgetting to take the travel log form & 0 & 5 & 0 & - \\
\hline Failing to record information about a trip & 0 & 5 & 0 & - \\
\hline Failing to record an entire trip on the travel log & 1 & 4 & 0 & $\begin{array}{l}\text {-Subject } 3 \text {, one short trip to } \\
\text { grocery store }\end{array}$ \\
\hline $\begin{array}{l}\text { Uncomfortable/ bothersome to carry the GPS data- } \\
\text { logger? }\end{array}$ & 1 & 4 & 0 & $\begin{array}{l}\text {-bothersome when carrying } \\
\text { groceries }\end{array}$ \\
\hline $\begin{array}{l}\text { Bothersome to carry and record all trips, on the travel } \\
\text { log form? }\end{array}$ & 1 & 4 & 0 & - \\
\hline GPS unit interfere with movements or activities? & 2 & 3 & 0 & $\begin{array}{l}\text {-accompanying friend had to wait } \\
2 \text { minutes as well } \\
\text {-had to remember to take it } \\
\text { everywhere }\end{array}$ \\
\hline $\begin{array}{l}\text { Bothered that someone knows about all of the } \\
\text { walking trips you made }\end{array}$ & 0 & 5 & 0 & - \\
\hline
\end{tabular}

None of the subjects reported that they failed to carry and switch on the GPS unit during the trips made. According to the survey form, two subjects failed to wait for the necessary two minutes at the start of trips. Subject 3 failed to wait the initial two minutes once, whereas Subject 4 failed twice, mentioning that they kept forgetting to do that 
during the initial part of the experiment. From analysis of the data presented earlier, it was observed that Subject 1 failed to wait for the required two minutes on one occasion but failed to mention this in the survey responses. All subjects reported that they remembered to carry the travel log form for their trips. Only one subject forgot to record an entire trip, which was a short trip to the grocery store, in the travel log, although the subject did carry the GPS unit along. The same subject thought that it was bothersome to carry the GPS unit along with the groceries in both hands. One subject considered carrying the log and recording trips as bothersome. Forty percent of the subjects thought that the GPS unit interfered with their activities or movements. None of the subjects in this experiment reported that they were bothered about someone knowing information about the trips they made.

The analysis of the GPS data collected for the trips presented some travel information which may be inaccurate and thus may not be reliable. For example, for the first trip analyzed, there was a high average speed indicated in one portion of the trip. For the second trip, the profile obtained by plotting elevations did not reflect actual conditions and, thus, was not considered reliable. In this analysis, however, the speeds were both reasonable and consistent. These inconsistencies may pose a problem when the results are used for engineering or for design purposes. Considering the positive characteristics of the hand-held GPS unit, it was observed that the device was small, light-weight and easy to use. The subjects did not find it uncomfortable or bothersome to carry. The data recorded by the unit gave a good idea of the route selected by the user for a trip, when data was plotted on a street-map of the vicinity. 
It should also be noted that these results have been obtained from experimental data and thus can be biased considering that subjects who volunteered for the experiment were all college-age males. Different results may be expected if females or senior citizens were also included as subjects. Thus, further study is recommended in order to have a more representative sample and to examine effects of age and gender on results.

Various observations were made after analysis of data obtained from the handheld GPS unit experiment. It was concluded that the start-up time of the GPS device may present a major problem for recording data. This problem, also defined as cold-start phenomenon (described in Chapter 3), may result in no data being recorded for the first few minutes of a trip and, in some instances, even a whole trip might be left unrecorded. Thus, device users need to wait at the starting point for a few minutes in order to let the hand-held GPS unit configure the satellite communication. As would be expected, subjects are not generally willing to wait at the start of each trip. Also, at locations surrounded by relatively tall buildings, the satellite signal reception of the GPS unit is affected. The device may not record data or may record incorrect data resulting in position errors or no position reading in some instances. It was also noted that in some cases delays of shorter duration may not be recorded.

The device may interfere with the activities of the user since the GPS unit cannot be covered by any clothing or kept in a pocket. Another concern with the unit is that the device has to be held upright for it to communicate properly with the satellites, since the antenna of the hand-held GPS unit is located in the top portion of the device. Analysis of trip data recorded for one of the trips showed that the elevations were incorrect and thus it is recommended that the analyst not rely on elevation data for engineering purposes. 
In this experiment, a travel log form was used in order to confirm the data obtained from the GPS unit. Even though most of the subjects did not find it uncomfortable to carry and record data at the start and end of each trip, it is not considered feasible to use the travel log form for longer duration applications. After analysis of the data obtained, it is recommended that results be shared with the subject in a post-experiment discussion as it is helpful to be one-on-one with the subject to answer questions. However, it is recognized that this is time-consuming.

After looking at the various issues surrounding its use, it was concluded that the hand-held GPS unit has potential for pedestrian data collection for certain situations and specific conditions. The unit may be used to collect data with a large number of subjects since the cost involved is reasonable. The device should not be used in urban areas where there are tall buildings since this could affect the data obtained as the urbancanyon effect, mentioned in Chapter 3, can come into play. The data obtained for certain pedestrian travel information such as origins and destinations, route selected and speed are considered reliable. Thus, it is concluded that it is feasible to use the hand-held GPS unit to collect pedestrian travel data for planning purposes but not for design purposes. The unit should be used under the following conditions for obtaining most reliable pedestrian information:

- The unit should not used in urban areas where there are tall buildings (such as downtown areas).

- The unit should not be used for studies where elevation data is required, as the device cannot be considered reliable.

- The device should not be used in areas where there is thick tree cover. 
- The GPS unit can be used to collect data with a large number of subjects.

- The unit should only be used for planning purposes and not design purposes as data can be unreliable and inaccurate.

- Although it may not be feasible in practical applications, the GPS unit should be used along with a travel log form confirming the trips made by a subject.

\subsection{Evaluation of Operational Considerations - Wearable GPS Data-Logger}

Following brief instructions, the wearable GPS data-logger and the travel log form were given to 5 subjects for periods of 24 hours each. The data collected using the data-logger was downloaded to a computer for analysis. Along with the data logger, the subjects also returned their completed travel data log form which provided information about their trips. When they returned the data logger and travel log forms, they were asked to complete a brief questionnaire about the experience. The data from the five subjects was compiled and analyzed to determine various types of trip information such as elevations, origin and destination, trip duration, speed, delays enroute and route selected. The procedure followed for the analysis was described in Chapter 3.

From the travel log form given to each subject, various trip information such as street address and time at origin, street address and time at destination and mode of travel were obtained for all trips made during the 24-hour period for all subjects. The trip information provided on the travel log form was compared to the trips shown on the map after data points were plotted. Any trip which a subject made during the day but failed to record on the travel $\log$ form was identified in this fashion. Similarly identified were situations where the subject recorded a trip on the travel log form but forgot to switch on the wearable GPS data-logger. The comparison also showed whether the subject 
recorded the time and address at origin and destination correctly. A summary of the trip information for each subject, during the course of the experiment, is shown in Table 37. The table presents information about the number of trips made by each subject, average speed of all trips, average duration of each trip, average distance walked and the total delay involved during the experiment.

Table 37 - Summary of All Walking Trips for All Subjects Obtained Using a Wearable GPS Data-Logger

\begin{tabular}{|c|c|c|c|c|c|}
\hline & $\begin{array}{c}\text { No. of } \\
\text { Walking } \\
\text { Trips } \\
\end{array}$ & $\begin{array}{c}\text { Average } \\
\text { Speed per } \\
\text { Trip (fps) } \\
\end{array}$ & $\begin{array}{c}\text { Average } \\
\text { Duration of } \\
\text { Each Trip } \\
\end{array}$ & $\begin{array}{c}\text { Average Distance } \\
\text { Walked per Trip } \\
\text { (miles) }\end{array}$ & $\begin{array}{c}\text { Average } \\
\text { Delay Per } \\
\text { Trip } \\
\end{array}$ \\
\hline \multicolumn{6}{|l|}{ Subject } \\
\hline 1 & 10 & 3.2 & $6.8 \mathrm{~min}$ & 0.25 & $5 \mathrm{sec}$ \\
\hline 2 & 7 & 3.6 & $7 \mathrm{~min}$ & 0.28 & $9.2 \mathrm{sec}$ \\
\hline 3 & 6 & 3.2 & $9.7 \mathrm{~min}$ & 0.35 & $11.6 \mathrm{sec}$ \\
\hline 4 & 6 & 3.9 & $5.9 \mathrm{~min}$ & 0.25 & $5 \mathrm{sec}$ \\
\hline 5 & 8 & 4.1 & $7 \mathrm{~min}$ & 0.32 & $13.7 \mathrm{sec}$ \\
\hline Grand Average & 7 & 3.6 & $7.3 \mathrm{~min}$ & 0.30 & $8.9 \mathrm{sec}$ \\
\hline
\end{tabular}

From Table 37, it can be observed that the total number of walking trips made by each subject during the 24-hour duration ranged from 6 to 10 trips, with an average speed per trip varying from $3.2 \mathrm{fps}$ to $4.1 \mathrm{fps}$. The average duration of each trip varied from 5.9 minutes for Subject 4 to almost 10 minutes for Subject 3, and the average distance walked varied from quarter of a mile to almost a third of a mile. The last column shows the average delay per trip. Delay has been defined as the duration of time during which the speed is zero even when the GPS unit has a strong satellite signal. The average delay per trip has been calculated by dividing the total delay of all trips for each subject by number of trips made by that subject in the 24-hour duration of the experiment. This delay ranged from a minimum of 5 seconds to a maximum of 13.7 seconds per trip. 
Considering Subject 1, the trips recorded on the travel log form were compared with the data points obtained from the GPS unit. A typical travel log form with the data recorded by Subject 1 is shown in Table 38. In an ideal situation, the travel log would have been presented verbatim, but in order to insure privacy of the subjects, and to give a better description of the origins and destinations, the travel log presented here is the version edited by the researcher.

Table 38 - Typical Data Recorded by Subject 1in the Travel Log Form in Wearable GPS Data-Logger Experiment

\begin{tabular}{|c|c|}
\hline Trip 1 (Day 1) & Trip 6 \\
\hline Origin- Mineral Resources Building & Origin- PRT Beechurst \\
\hline Origin time - 1515 & Origin time -0814 \\
\hline Mode- Walk & Mode- Walk \\
\hline Destination- Coliseum Parking Lot & Destination- St. John's Church \\
\hline Destination time- 1525 & Destination time- 0819 \\
\hline $\begin{array}{rr}\text { Trip } 2 \\
\end{array}$ & $\begin{array}{r}\text { Trip } 7 \\
\end{array}$ \\
\hline Origin- Engineering Sciences & Origin- St. John's Church \\
\hline Origin time - 1722 & Origin time - 0820 \\
\hline Mode- Walk & Mode- Walk \\
\hline Destination- Engineering PRT Station & Destination- Hodges Hall \\
\hline Destination time- 1728 & Destination time- 0822 \\
\hline $\begin{array}{r}\text { Trip } 3 \\
\end{array}$ & Trip 8 \\
\hline Origin- PRT Beechurst Station & Origin- Towers Residence Hall \\
\hline Origin time -1736 & Origin time -0939 \\
\hline Mode- Walk & Mode- Walk \\
\hline Destination- St. John's Church & Destination- Mineral Resources Building \\
\hline Destination time- 1742 & Destination time- 0946 \\
\hline Trip 4 & Trip 9 \\
\hline Origin- St. John's Church & Origin- Mineral Resources Building \\
\hline Origin time -1758 & Origin time -1152 \\
\hline Mode- Walk & Mode- Jog \\
\hline Destination- Hodges Hall & Destination- Allen Hall \\
\hline Destination time- 1800 & Destination time- 1159 \\
\hline Trip 5 (Day 2) & $\begin{array}{r}\text { Trip } 10 \\
\end{array}$ \\
\hline Origin- Home & Origin- Allen Hall \\
\hline Origin time -0751 & Origin time -1308 \\
\hline Mode- Walk & Mode- Jog \\
\hline Destination- PRT at Engineering & Destination- Mineral Resources Building \\
\hline Destination time- 0806 & Destination time- 1314 \\
\hline
\end{tabular}

It was observed that the times recorded on travel log form for the individual trips matched very well with the GPS data. There was a variation of only one to two minutes for some of the entries, which would be expected. When comparing the information on origins/destinations from the travel log to the data points plotted on map of Morgantown, 
it was observed that the addresses provided for identification matched closely with the traveled routes. Note that the intermodal connections are not shown explicitly and the data obtained shows only the walking trips. It was inferred that there were at least two inter-modal trips involving motorized means of travel. For example, Trip 2 ended at a PRT station at the Engineering Campus of WVU, while Trip 3 began at a different PRT station near downtown, i.e. several miles away. Given the elapsed time, it can be reasonably concluded that the trip was not made by walking. The most likely mode was the PRT but this cannot be verified from the data.

Two different trips were considered for detailed analysis and presentation. Since the purpose of the study was to address device capability, trips were selected which were longer than others, included street crossing and varied topography, and were made at different times of the day. Once the two trips were selected, the records for these trips were analyzed to obtain travel data. Travel data refers to information about a trip, including time-of-day of the trip, speeds, delays enroute, origins/destinations, route selected, trip length and trip purpose.

Figure 27 shows the plotted data-points of two trips on a street map of the Morgantown area. This map was mainly a campus map (obtained from the West Virginia University's GIS Technology Center), which showed the various campuses of West Virginia University in Morgantown and details of streets in the vicinity of the campuses. The process for plotting the data points on a map has been described earlier in Chapter 3 . Besides a street map, users can also use contour maps, USGS topographic maps and aerial photographs to plot points. 


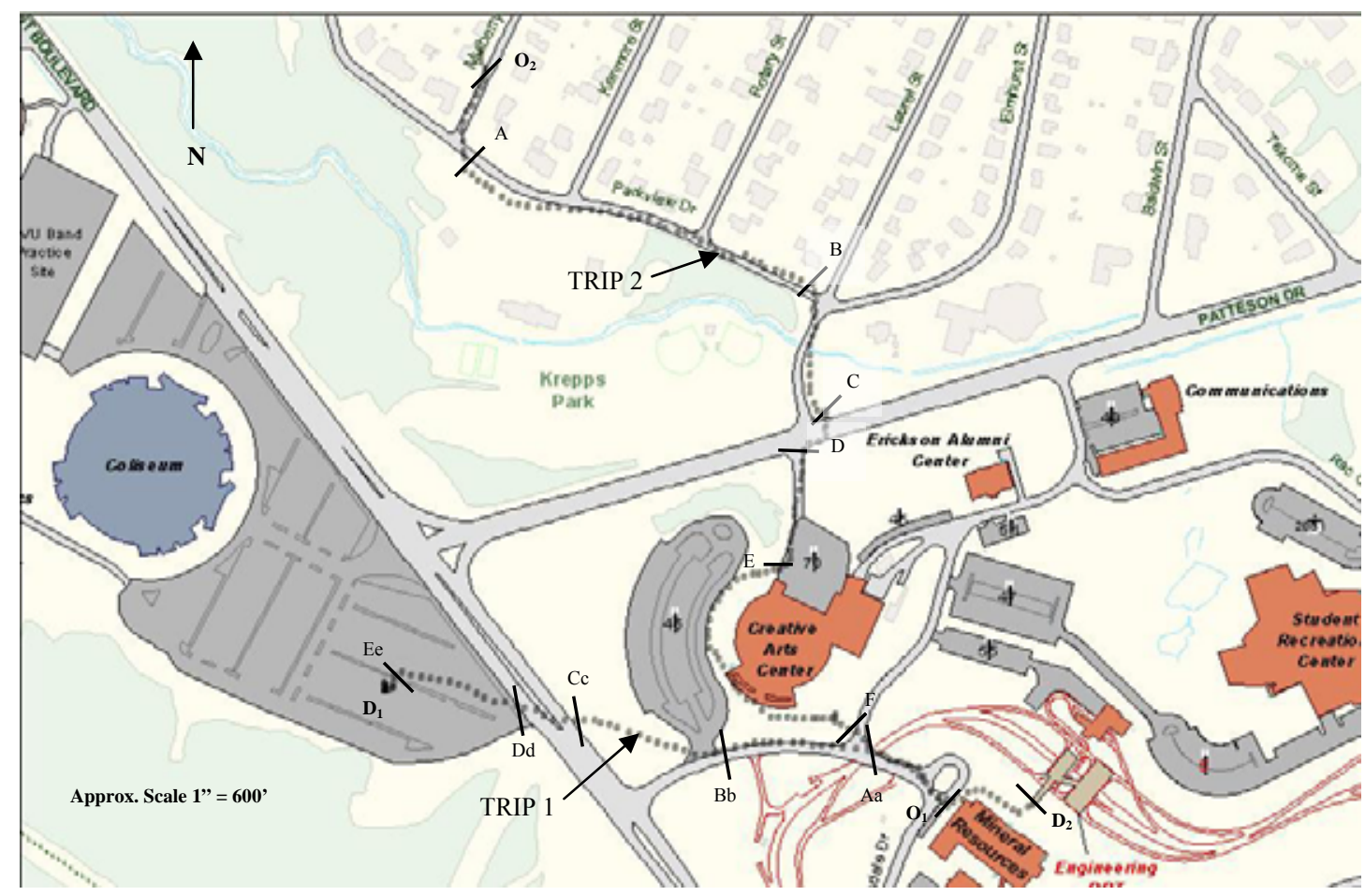

Figure 27: GPS Data for Two Trips in the Vicinity of the WVU Campus for Subject 1, Obtained Using Wearable GPS Data-Logger and Plotted on Arcview GIS Software

The two trips were made at different times. Trip 1, which took place during midafternoon, started at the Mineral Resources Building on the Evansdale Campus and ended at the parking lot adjacent to the WVU Coliseum. The route along this trip is primarily downhill. The pedestrian facility, i.e., a sidewalk, is wide and in good condition till the point where the subject starts to cross Monongahela Boulevard. There is no marked crosswalk to cross Monongahela Boulevard at the point where the subject made the crossing and the route taken is identical to that used by a large number of other students who cross at this location.

Trip 2 was made during early morning and started on a residential street near Krepps Park and ended at the Engineering Personal Rapid Transit (PRT) station next to the Mineral Resources Building on Evansdale Campus. The route along this trip is both uphill and level at places. A sidewalk was present along most of the trip route and was in 
good condition. The subject crossed the Patteson Drive at a location where there is no marked crosswalk, and had to find a gap in traffic. Each of the two trips shown in Figure 27 has been divided into sections of unequal length. The starting and ending points of each section are identified by alphabetic characters.

From the above plot, many types of travel information such as origin/destination, elevations (and consequently gradients), speed, trip duration, route selection and delays can be obtained. For the first trip analyzed, i.e., Trip 1, an elevation versus time profile was prepared, as shown in Figure 28.

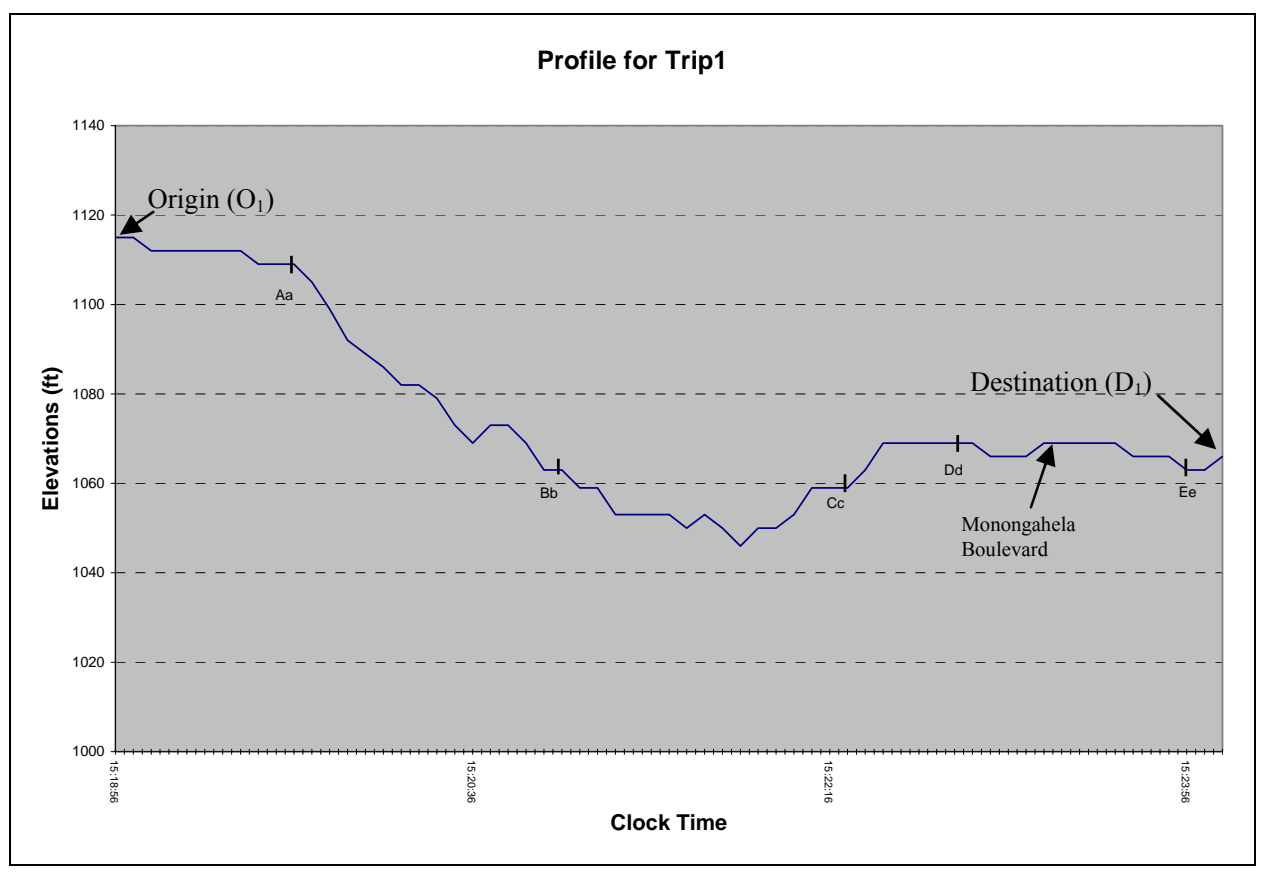

Figure 28: Profile for Trip 1 Obtained from Wearable GPS Data-Logger

From Figure 28, it can be observed that the profile is downgrade until about twothirds of the way between B and C and then increases in elevation. There is not much variation in the elevation after point $\mathrm{C}$ to the end of trip. The subject crosses Monongahela Boulevard between points C and D. The profile in the figure seems to 
reflect reality. The starting point is at the top of a hill and does go down initially till the Boulevard and then rises slightly before becoming fairly level in the parking lot.

The graph presented in Figure 29 shows the speed profile for the trip and was obtained by plotting the recorded speeds versus distance along the trip.

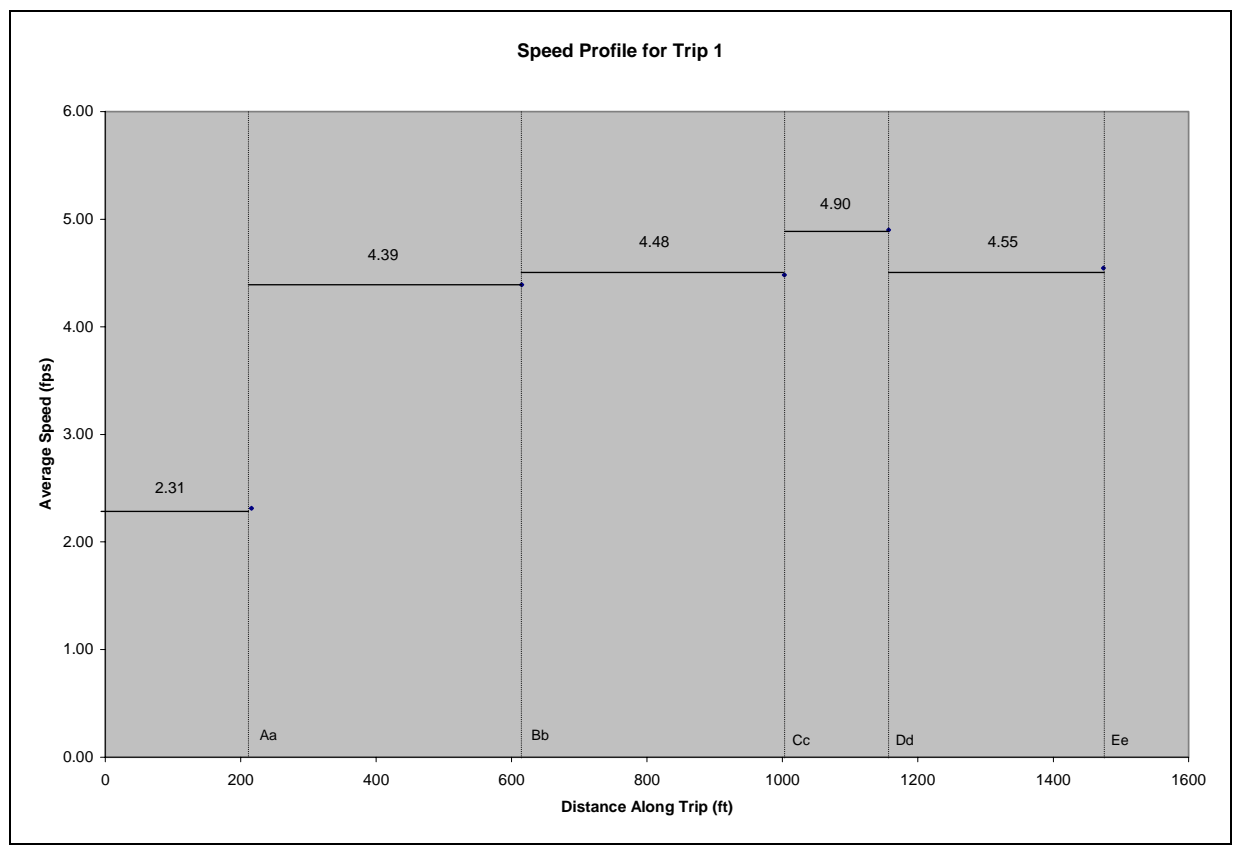

Figure 29: Speed Profile for Trip 1 Obtained from Wearable GPS Data-Logger

From Figure 29, it can be observed that the average speed of the subject from point $\mathrm{A}$ to point $\mathrm{E}$ was about 4.5 fps. The average speed from origin to point $\mathrm{A}$ was low because of a delay recorded in that section; the speed during that particular time record was zero, which was evident from the data downloaded from the wearable GPS datalogger, shown in Table 39. Table 39 shows that there was a delay of about 10 seconds during the early part of the trip as the speed of the subject was recorded as zero. Considering the duration of time the speed was zero, it was hypothesized that the delay was due to a short social interaction between the subject and another individual(s) or the subject stopped to wait for a gap in traffic before crossing the street. 
The subject crossed Monongahela Boulevard between points C and D. Note that

the average speed in this section was greater than the average speeds in other sections,

suggesting that the subject walked faster during crossing. Data shows that the subject

must have relatively quickly found a gap in traffic acceptable for crossing Monongahela

Boulevard, as there was no zero speed in the data at that instant, i.e., at point Cc.

Table 39 - Sample Data Obtained from Wearable GPS Data-Logger Unit for Trip 1 for Subject 1

\begin{tabular}{|c|c|}
\hline RECORD,FLAG,DATE,TIME,LAT,LONG,SPEED,HEAD,ALTITUD & DOP,SATS \\
\hline 29,A,02/22/05,15:18:51,39.64698,-79.97418,000.0,348,01118,04.6,04 & \\
\hline 30,A,02/22/05,15:18:56,39.64702,-79.97427,001.3,348,01115,05.9,04 & \\
\hline $31, \mathrm{~A}, 02 / 22 / 05,15: 19: 01,39.64705,-79.97432,001.7,307,01115,04.7,04$ & \\
\hline $32, \mathrm{~A}, 02 / 22 / 05,15: 19: 06,39.64708,-79.97435,000.0,000,01112,04.8,04$ & Delay \\
\hline $33, \mathrm{~A}, 02 / 22 / 05,15: 19: 11,39.64713,-79.97442,000.0,000,01112,04.8,04$ & Delay \\
\hline $34, \mathrm{~A}, 02 / 22 / 05,15: 19: 16,39.64720,-79.97448,000.0,000,01112,04.1,04$ & Delay \\
\hline $35, \mathrm{~A}, 02 / 22 / 05,15: 19: 21,39.64723,-79.97453,002.1,310,01112,04.1,04$ & \\
\hline $36, \mathrm{~V}, 02 / 22 / 05,15: 19: 26,39.64727,-79.97458,002.1,307,01112,04.1,00$ & \\
\hline $37, \mathrm{~A}, 02 / 22 / 05,15: 19: 31,39.64730,-79.97465,002.4,301,01112,04.2,03$ & \\
\hline $38, \mathrm{~A}, 02 / 22 / 05,15: 19: 36,39.64732,-79.97472,002.4,295,01109,04.8,04$ & \\
\hline 39,A,02/22/05,15:19:41,39.64735,-79.9748,002.7,302,01109,04.1,04 & \\
\hline 40,A,02/22/05,15:19:46,39.64738,-79.97487,002.6,294,01109,04.3,04 & Point Aa \\
\hline 41,A,02/22/05,15:19:51,39.64740,-79.97498,002.7,290,01105,04.7,04 & \\
\hline 42,A,02/22/05,15:19:56,39.64742,-79.97508,002.9,289,01099,03.7,05 & \\
\hline 43,A,02/22/05,15:20:01,39.64742,-79.9752,002.9,284,01092,03.6,05 & \\
\hline $44, \mathrm{~A}, 02 / 22 / 05,15: 20: 06,39.64743,-79.9753,002.9,282,01089,03.5,05$ & \\
\hline 45,A,02/22/05,15:20:11,39.64743,-79.9754,003.1,281,01086,03.5,05 & \\
\hline 46,A,02/22/05,15:20:16,39.64743,-79.97548,003.1,279,01082,03.5,05 & \\
\hline 47,A,02/22/05,15:20:21,39.64745,-79.97557,003.2,276,01082,03.6,05 & \\
\hline $48, \mathrm{~A}, 02 / 22 / 05,15: 20: 26,39.64745,-79.97565,003.1,272,01079,03.6,05$ & \\
\hline 49,A,02/22/05,15:20:31,39.64745,-79.97577,003.4,270,01073,02.8,05 & \\
\hline 50,A,02/22/05,15:20:36,39.64745,-79.97585,003.3,273,01069,02.4,05 & \\
\hline 51,A,02/22/05,15:20:41,39.64745,-79.97592,002.9,267,01073,03.3,05 & \\
\hline 52,A,02/22/05,15:20:46,39.64745,-79.976,002.9,269,01073,03.6,05 & \\
\hline 53,A,02/22/05,15:20:51,39.64743,-79.97608,002.7,268,01069,03.6,05 & \\
\hline $54, \mathrm{~A}, 02 / 22 / 05,15: 20: 56,39.64742,-79.97617,002.8,265,01063,03.6,05$ & \\
\hline 55,A,02/22/05,15:21:01,39.64740,-79.97627,002.9,265,01063,03.7,05 & Point $\mathrm{Bb}$ \\
\hline 56,A,02/22/05,15:21:06,39.64738,-79.97635,003.2,253,01059,05.2,05 & \\
\hline $57, \mathrm{~V}, 02 / 22 / 05,15: 21: 11,39.64737,-79.97643,003.1,254,01059,04.4,00$ & \\
\hline 58,A,02/22/05,15:21:16,39.64737,-79.97653,003.1,259,01053,04.3,04 & \\
\hline 59,A,02/22/05,15:21:21,39.64740,-79.97662,003.2,264,01053,04.5,04 & \\
\hline 60,A,02/22/05,15:21:26,39.64742,-79.9767,003.2,269,01053,03.7,05 & \\
\hline 61,A,02/22/05,15:21:31,39.64743,-79.97678,003.2,273,01053,03.6,05 & \\
\hline $62, \mathrm{~A}, 02 / 22 / 05,15: 21: 36,39.64745,-79.97687,003.1,276,01050,03.6,05$ & \\
\hline 63,A,02/22/05,15:21:41,39.64747,-79.97695,002.7,277,01053,03.6,05 & \\
\hline $64, \mathrm{~A}, 02 / 22 / 05,15: 21: 46,39.64750,-79.97703,002.8,279,01050,03.6,05$ & \\
\hline $65, \mathrm{~A}, 02 / 22 / 05,15: 21: 51,39.64752,-79.97712,002.9,279,01046,03.7,05$ & \\
\hline $66, \mathrm{~A}, 02 / 22 / 05,15: 21: 56,39.64755,-79.97722,003.1,278,01050,02.1,06$ & \\
\hline 67,A,02/22/05,15:22:01,39.64757,-79.9773,003.2,277,01050,02.4,06 & \\
\hline 68,A,02/22/05,15:22:06,39.64758,-79.97737,003.1,278,01053,02.2,06 & \\
\hline 69,A,02/22/05,15:22:11,39.64760,-79.97743,002.9,274,01059,02.0,06 & \\
\hline
\end{tabular}




\begin{tabular}{|l|}
\hline 70,A,02/22/05,15:22:16,39.64760,-79.97752,003.1,262,01059,02.7,06 \\
\hline 71,A,02/22/05,15:22:21,39.64760,-79.9776,002.9,265,01059,02.9,06 Point Cc \\
\hline 72,A,02/22/05,15:22:26,39.64760,-79.9777,003.3,264,01063,02.8,06 \\
\hline 73,A,02/22/05,15:22:31,39.64760,-79.9778,003.4,264,01069,03.2,06 \\
\hline 74,A,02/22/05,15:22:36,39.64763,-79.97788,003.2,277,01069,01.9,06 \\
\hline 75,A,02/22/05,15:22:41,39.64767,-79.97795,003.3,284,01069,01.8,06 \\
\hline 76,A,02/22/05,15:22:46,39.64768,-79.97805,003.4,281,01069,02.3,06 \\
\hline 77,A,02/22/05,15:22:51,39.64770,-79.97813,003.4,285,01069,02.4,06 \\
\hline 78,A,02/22/05,15:22:56,39.64772,-79.97823,003.5,279,01069,02.3,06 Dd \\
\hline 79,A,02/22/05,15:23:01,39.64772,-79.97833,003.5,279,01066,02.7,06 \\
\hline 80,A,02/22/05,15:23:06,39.64775,-79.97842,003.4,286,01066,01.9,06 \\
\hline 81,A,02/22/05,15:23:11,39.64778,-79.97848,003.3,290,01066,01.9,06 \\
\hline 82,A,02/22/05,15:23:16,39.64782,-79.97857,003.3,291,01069,02.0,06 \\
\hline 83,A,02/22/05,15:23:21,39.64785,-79.97865,003.3,292,01069,02.0,06 \\
\hline $84, \mathrm{~A}, 02 / 22 / 05,15: 23: 26,39.64787,-79.97873,003.2,291,01069,02.1,06$ \\
\hline $85, \mathrm{~A}, 02 / 22 / 05,15: 23: 31,39.64788,-79.97882,003.3,288,01069,02.2,06$ \\
\hline $86, \mathrm{~A}, 02 / 22 / 05,15: 23: 36,39.64788,-79.9789,003.3,282,01069,02.5,06$ \\
\hline $87, \mathrm{~A}, 02 / 22 / 05,15: 23: 41,39.64790,-79.97898,003.3,280,01066,02.0,06$ \\
\hline $88, \mathrm{~A}, 02 / 22 / 05,15: 23: 46,39.64790,-79.97907,003.3,280,01066,02.3,06$ \\
\hline $89, \mathrm{~A}, 02 / 22 / 05,15: 23: 51,39.64793,-79.97915,003.2,284,01066,02.1,06$ \\
\hline 90,A,02/22/05,15:23:56,39.64790,-79.97922,002.6,268,01063,03.0,06 \\
\hline 91,A,02/22/05,15:24:01,39.64785,-79.97923,002.2,244,01063,02.4,06 \\
\hline 92,A,02/22/05,15:24:06,39.64782,-79.97923,001.7,244,01066,01.9,06 \\
\hline
\end{tabular}

From the data points obtained for the first trip, trip duration can be obtained by inference, by calculating the difference between the time at destination and the time of origin. For the first trip, travel time was calculated as 5 minutes and 10 seconds. The approximate length of trip was 0.3 mile.

There was one delay observed enroute. Delays can be inferred from the data points obtained from the GPS unit by looking at the speeds in the downloaded data. The points at which the speed is zero are considered as points of delay. Thus, using the data shown in Table 39, the delay time was calculated (i.e. 15:19:16 - 15:19:06 $=10$ seconds). In the first trip, a delay of about 10 seconds was observed, which could have been due to some social interaction with another individual or waiting for an acceptable gap in traffic at a road crossing.

The data points obtained from the second trip indicated that the total trip duration was 13 minutes and 1 second for an approximate distance of 0.65 mile. Table 40 presents 
the data collected by the wearable GPS data-logger for the second trip. It can be seen from Table 40 that between points $\mathrm{C}$ and $\mathrm{D}$, the subject had to cross Patteson Drive for which the subject slowed down, probably to find a gap in vehicular traffic on this wide, high-volume roadway (note that there is a center two-way left-turn lane at this location).

Table 40 - Sample Data Obtained from Wearable GPS Data-Logger Unit for Trip 2 for Subject 1

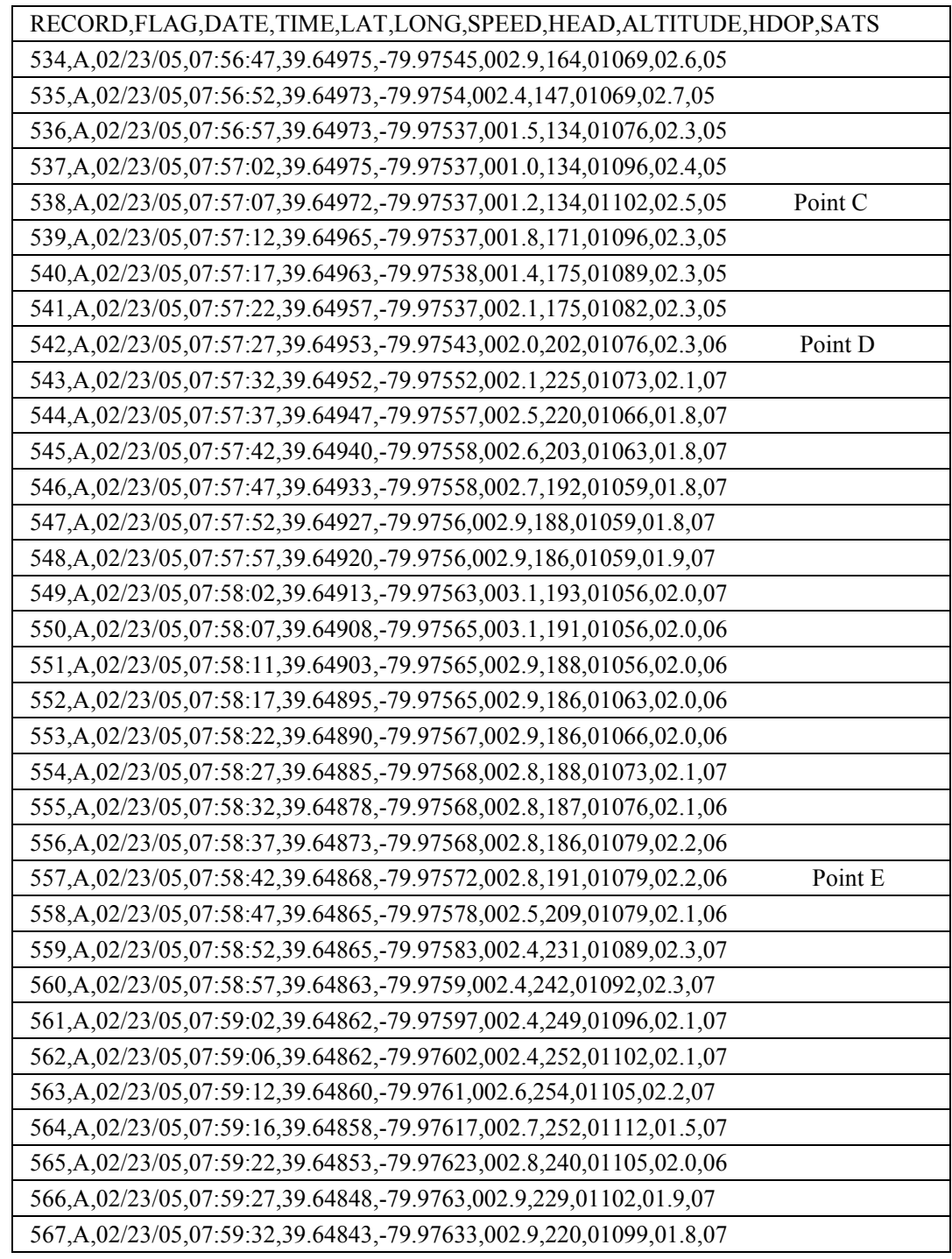

The route selected can be seen on the plot of the trip in Figure 27. The profile obtained by plotting elevations against the clock time for the second trip is shown in Figure 30 . It can be seen that the trip was mostly upgrade with the highest point at the end of trip, which is at the Engineering Station of the Personal Rapid Transit (PRT) 
station on the Evansdale campus, situated at a higher elevation than rest of the points of the trip. Figure 30 shows that Trip 2 was both uphill and downhill and that there were three peaks in elevations at different instances.

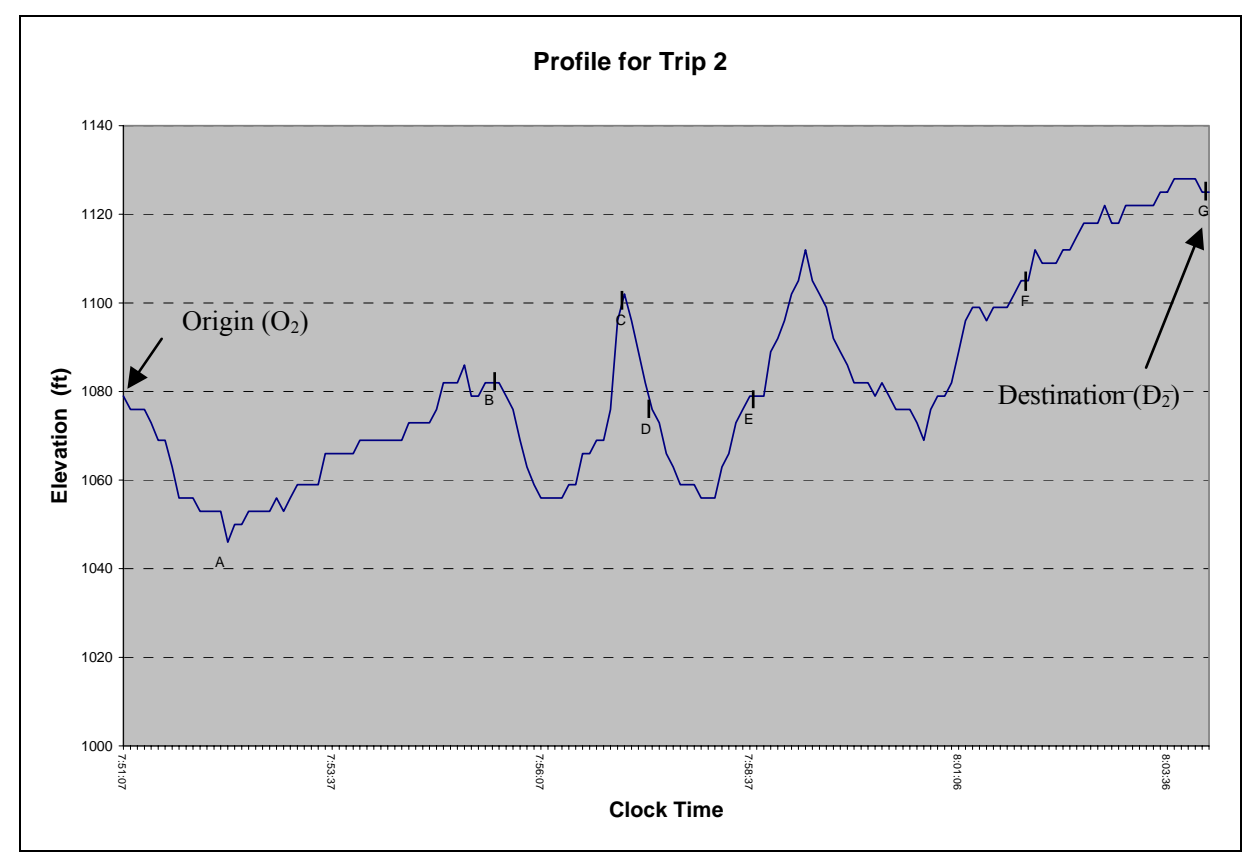

Figure 30: Profile for Trip 2 Obtained from Wearable GPS Data-Logger

Figure 30 also shows that the elevation of Point C, i.e., before crossing Patteson Drive, was much greater than the elevation at Point $\mathrm{D}$, i.e., after crossing Patteson Drive; there is a drop in elevation from point $\mathrm{C}$ to point $\mathrm{D}$. At point $\mathrm{C}$, the elevation, as recorded by the wearable GPS data logger, was 1102 feet and at point D, it was recorded as 1076 feet. That was a decrease in elevation of 26 feet from one side of the street to the other side. This change in elevation does not agree with the actual situation at that location, and thus does not reflect reality.

From the speed profile shown in Figure 31, it can be seen that the average speeds on each of the sections varied depending on factors such as topography, delays enroute (such as slowing or stopping to cross a road), and type of facility. The subject's highest 
speed (4.2fps) occurred between A and B and F and G. This was attributable to the relatively level gradient and good surface characteristics on these sections. The subject crossed Patteson Drive between points $\mathrm{C}$ and $\mathrm{D}$, and thus it can be inferred that the average speed for that stretch is lower because the subject was essentially stopped or walked slowly while trying to find a gap in the traffic.

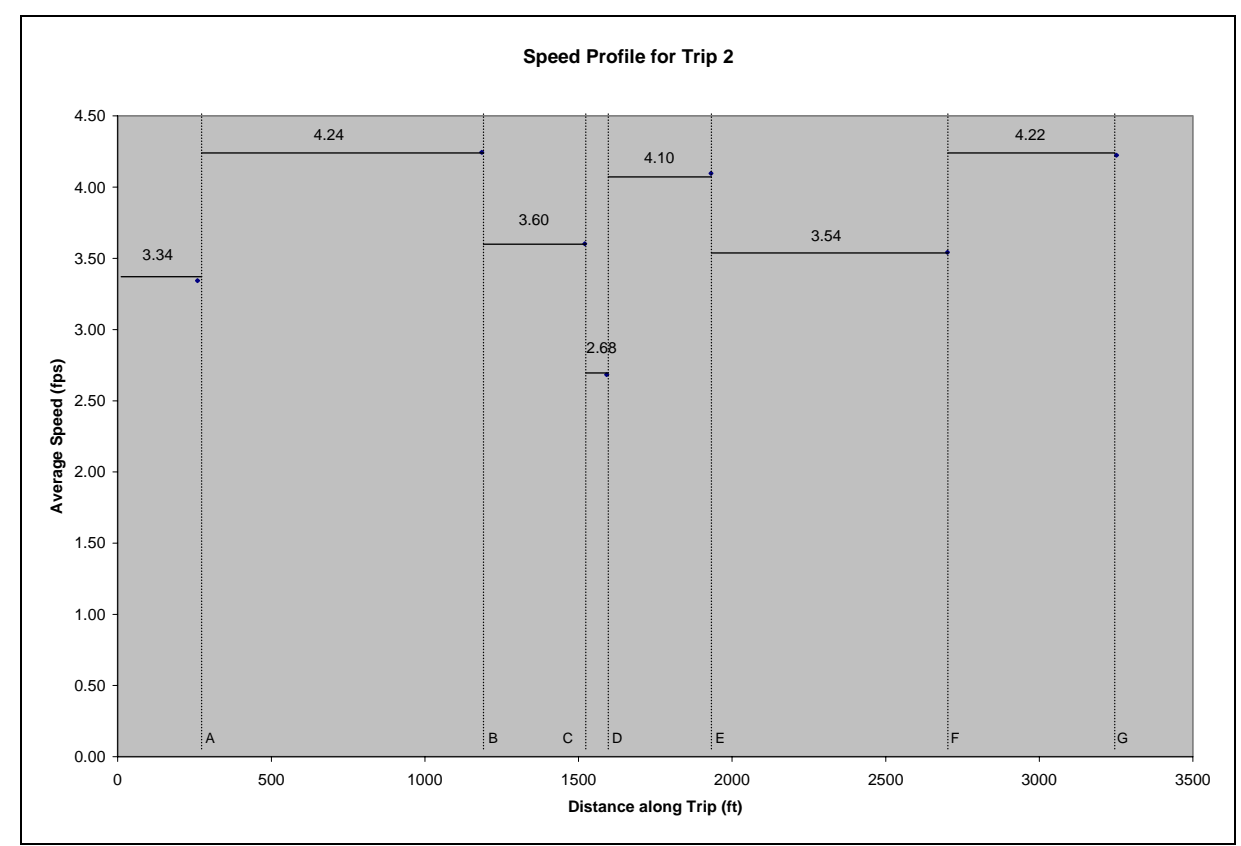

Figure 31: Speed Profile for Trip 2 Obtained from Wearable GPS Data-Logger

In similar manner, trip information can be obtained for all trips made by each subject. In addition to gathering and determining various travel characteristics of individuals, this information can also be used for improving the existing pedestrian facilities on the route used more often by pedestrians. It can also be used for accommodating pedestrian signals at intersections where pedestrians cross a street, if warranted. Thus, the information obtained from the GPS data can be used for traffic planning purposes and for upgrading various facilities for the pedestrians. 
This experiment involved costs for the wearable GPS data-logger, and for a computer to which the data would be downloaded. The data-logger, although found to be an accurate and reliable data collection device, may prove to be expensive for collecting sample data for a large population since each wearable GPS data-logger costs about $\$ 900$.

The time involved in processing the data includes downloading data to a computer and overlaying the data points on a street map of the area. The procedure followed for post-processing of collected data has been explained in detail in Chapter 3. The postprocessing, i.e., downloading, analyzing and overlaying procedure for 8 to 12 trips recorded by a subject required about 2.5 to 3 hours for each sample subject. The postprocessing also included analyzing the responses to the questionnaire. The educational requirement for this experiment and consequently data processing includes a minimum of technician level education in the field of transportation, some basic knowledge of computers and basic information about GPS technology. A formal training course in GIS and software working on GIS platform would be helpful but not necessary. The person conducting the experiments should have good analytical skills to interpret the data collected and should be comfortable with high-school mathematics.

The questionnaires were analyzed in order to address concerns about user response/acceptability to the device and the experience. A summary of the questionnaire results is presented in Table 41. It can be observed that subjects reported that they followed the correct procedure for data collection, as described in the instructions. Subjects reported that they recorded the trips on the travel log form correctly, without failing to record trips and their origins/destinations. This fact was also confirmed from 
the travel log data. However, it cannot be independently verified if the subjects actually took the device with them on every walking trip.

Table 41- Summary of Subject Responses to Questionnaire on Wearable GPS Data-Logger

\begin{tabular}{|c|c|c|c|c|}
\hline Question & Yes & No & Blank & Comments \\
\hline Failing to take the GPS data logger & 0 & 5 & 0 & - \\
\hline Failing to switch on the GPS data logger? & 0 & 5 & 0 & - \\
\hline Forgetting to wait the two minutes during start-up & 1 & 4 & 0 & $\begin{array}{l}\text {-Once, for a negligible walking } \\
\text { trip }\end{array}$ \\
\hline Forgetting to take the travel log form & 0 & 5 & 0 & - \\
\hline Failing to record information about a trip & 0 & 5 & 0 & - \\
\hline Failing to record an entire trip on the travel log & 1 & 4 & 0 & - \\
\hline $\begin{array}{l}\text { Uncomfortable/ bothersome to carry the GPS data- } \\
\text { logger? }\end{array}$ & 4 & 1 & 0 & -Only when jogging \\
\hline $\begin{array}{l}\text { Bothersome to carry and record all trips, on the travel } \\
\text { log form? }\end{array}$ & 1 & 4 & 0 & - \\
\hline GPS unit receiver ever slip off shoulders? & & $\begin{array}{l}\text { RARE } \\
\text { DCC } \\
\text { TREC }\end{array}$ & & - Not at all \\
\hline $\begin{array}{l}\text { GPS data-logger affected physical/personal } \\
\text { appearance? }\end{array}$ & 2 & 3 & 0 & - \\
\hline GPS unit interfere with movements or activities? & 4 & 1 & 0 & $\begin{array}{l}\text {-Bounced a lot while jogging } \\
\text {-accompanying friends had to } \\
\text { wait } 2 \text { minutes as well } \\
\text {-could not run without the unit } \\
\text { falling off }\end{array}$ \\
\hline $\begin{array}{l}\text { Bothered that someone knows about all of the } \\
\text { walking trips you made }\end{array}$ & 0 & 5 & 0 & - \\
\hline
\end{tabular}

None of the subjects reported that they failed to switch on the GPS unit during the trips made. According to the survey form, only one subject failed to wait for the necessary two minutes at the start of trip.

Eighty percent of the subjects thought that the unit was uncomfortable to wear for walking trips, whereas only one subject considered recording of trips in the travel log 
form as bothersome. This latter result was unexpected. Majority of the subjects did not think that the GPS unit affected their physical/personal appearance. This result may be biased since all subjects were males. Perceptions might be different if female subjects were included in the sample. Almost all subjects thought that the GPS unit interfered with their activities, such as jogging and other recreational activities. None of the subjects in this experiment reported that they were bothered about someone knowing information about the trips they made. It is not clear that this question is meaningful here since subjects were free to not use the device in specific trips.

The results obtained after analyzing the data obtained from wearable GPS datalogger and from the travel log form show that the data recorded by the device is fairly accurate and reliable. The results obtained after analysis of data can be used for planning purposes but not for design purposes. For example, the wearable GPS data-logger can be used to identify where a mid-block crosswalk is needed, but cannot be used for designing new pedestrian facilities like sidewalks. The size of the data-logger and the cost are drawbacks to its utility. Thus, it may be more useful for studies where the population and corresponding sample size requirements are small, so that cost of providing data collection devices (i.e., the data-logger) can be within budget.

It should be noted that these results have been obtained from a small, homogenous sample of participants, i.e., college-age males. Although the focus of this effort was to evaluate the process rather than the results, it is recognized that there is bias in the sample even from a process standpoint. For example, different results would be expected if females were also subjects for this experiment. Other age-groups, such as older individuals, were not represented in the sample size and, thus, results are biased towards 
age-group 20-25, since all subjects are in that age range. Thus, further study is recommended in order to have a more representative sample and to examine effects of age and gender on results.

Various observations were made after analysis of results obtained from the wearable GPS data-logger experiment. It was observed that the required start-up time of the GPS data-logger device is a significant limitation for actual data collection. This requirement, also defined as cold-start phenomenon (described in Chapter 3), may result in no data being recorded for the first few minutes of a trip and at some instances even a whole trip might be left unrecorded. Thus, the device user needs to wait at the trip starting point for a few minutes in order to let the data-logger configure the satellite communication. It is anticipated that subjects will be unwilling/unable to wait at the start of every trip.

Also, in places surrounded by relatively tall building, the satellite signal reception of the GPS unit is affected, i.e., the so-called urban canyon effect. The device may not record data or may record incorrect data resulting in position errors or no position reading at some instances. When the analyst analyzes the recorded information, he/she will get to know the erroneous or incorrect data. The analysis, for example, may consist of plotting the data on a map, analyzing table of data, calculations and plotting graphs, which would assist in drawing logical inferences.

As mentioned before, another drawback of the device is its size, which interferes with movements and activities of the device user. The user may also feel uncomfortable carrying the GPS unit hanging from the shoulder. The device may also act as a hindrance to the user since the receiver antenna cannot be covered by articles of clothing. The cost 
of the wearable GPS data-logger also makes it less feasible to use in case where a large sample size is needed.

Thus, after looking at the various issues surrounding its use, it is concluded that it is not feasible to use the wearable GPS data-logger for pedestrian data collection on a large scale. However, it does appear to have applications for collecting data where sample size requirements are small and in situations where the data is used for planning rather than design purposes. 


\section{Chapter 5}

\subsection{CONCLUSIONS AND RECOMMENDATIONS}

The study attempted to assess the feasibility of three different devices: (1) pedometer, (2) hand-held GPS unit and (3) wearable GPS data-logger in collecting pedestrian data for transportation planning and engineering purposes. A literature search was conducted to identify and evaluate data collection methods for both motorized and non-motorized travel and to find information on new technology available for pedestrian data collection. The review of literature found that procedures and devices for collecting vehicular travel data were far more developed than those for collecting non-motorized travel data. Although a number of methods for counting or determining the number of users on pedestrian facilities are available, there is a need for additional non-motorized user-related information such as trip origins and destinations, travel-time, travel speeds and delays.

Since the objective of this study was to assess the feasibility of the devices, several experimental designs were considered before finalizing the experimental procedures to evaluate device characteristics and operational considerations. Various types of information and data were obtained from experiments conducted using the three

devices. The analyses of the collected data showed some interesting results. In reviewing the results of this study, several points were concluded. These will be discussed below; recommendations, both for practitioners and researchers, follow. 


\subsection{Conclusions}

Wearable devices, such as pedometers and technologically advanced devices, such as GPS, have primarily been used for assessing physical activity of users and, for the latter, as navigation aids by outdoor enthusiasts. Only limited published information was found on using these devices as tools for pedestrian data collection. Thus, it seemed logical to explore the use of wearable devices (with and without GPS capability) to gather travel data, such as speed, duration of trip and origin/destination of the user, for nonmotorized modes. The assessment required answering questions about the devices' costs, data collection capabilities, accuracy, user acceptability and post-processing of obtained data.

The objective of this research study was to assess the feasibility of three devices in collecting pedestrian data for transportation engineering and planning purposes. The outcome of this research consisted of two sets of results. The first set provided information on the characteristics of the three devices, notably their accuracy and reliability. The second set addressed the operation and utility of the devices for collecting pedestrian travel data. Conclusions from each set are discussed below followed by overall conclusions.

Device Characteristics of Pedometer, Hand-held GPS Unit and Wearable GPS DataLogger

Results of the pedometer experiments showed that different surfaces and different gradients had no significant effect on stride length and, therefore, step count. This result differed from what had been intuitively expected. 
Social interaction had no significant effect on pedometer step count in this study. In the researcher's opinion, this was due to the inability of the experimental setup to replicate real-world situations. Data collected for the 'Effect of Other Pedestrian Traffic' experiment showed that under LOS A and LOS B, pedestrian stride length did not change significantly, due to the low density of pedestrian traffic. There was some indication of a change in stride length at LOS C but the data were not sufficient to draw any conclusions. The Hawthorne Effect could explain the results obtained. It must also be noted that the subjects were rather homogenous (primarily college-age males) and thus not representative of the population at large.

Assessing the hand-held GPS unit and the wearable GPS data-logger after analyzing them under four different field conditions showed mixed results. Both units worked well under high-voltage transmission lines and next to transmission towers. Under high-voltage facilities, no effect was observed on the availability of satellite signal, and data were recorded constantly. Similarly, limited testing showed that heavy rainfall did not affect the data recording capabilities of the two devices.

For the hand-held GPS unit under trees, there were a few instances where it lost connectivity to the satellites for significant periods of time and this loss of signal was not evident from the display screen. The wearable GPS data-logger unit had no problems connecting to satellites under dense tree canopy for both deciduous and coniferous trees.

When used near closely spaced buildings taller than 2 or 3 stories, both units seem to have a problem locating the satellites and, in many instances, data were not recorded for a significant time period. This is the so-called urban canyon effect reported in the literature. 
Based upon the analysis of the data collected from the two GPS devices under various experimental conditions, it was concluded that the two GPS devices can be used for collecting pedestrian travel data with certain restrictions. These restrictions involve various field conditions where the data collected may not prove to be fully reliable, mainly in the vicinity of closely spaced tall buildings and under thick forest covers. The same devices, when used in suburban or rural locations not under thick tree cover should provide reliable pedestrian travel data. Reliable travel data can be obtained only when there is direct communication between the GPS devices and satellites.

Operational Considerations of Pedometer, Hand-held GPS Unit and Wearable GPS Data-Logger

After carrying out the experiments on the three devices to ascertain their operational characteristics, various interesting results were obtained. These results were used in making various conclusions from an operational point of view.

For the pedometer, the experimental results indicate that even though it is lowcost, light and unobstrusive, the pedometer is not acceptable as a data collection device for pedestrian movement information. A primary reason is the absence of any data recording capability of the pedometer. The only data obtained is the step count which must be recorded manually (in a travel log form) along with origin/destination and clock time information. A travel log form is necessary to record trip information when using a pedometer. Thus, the results are susceptible to omissions, errors and manipulation. Furthermore, even if the step count is correct, only a rough estimate of distances and speeds can be obtained. Another disadvantage of using a pedometer is that it does not provide the researcher with information such as delays enroute or path of travel. 
In analyzing the two GPS units, some general limitations/problems, common to GPS units were observed. The start-up time of the GPS devices, also known as the coldstart phenomenon, is felt to be a major problem. It may result in no data being recorded for the first few minutes of a trip and, in some instances, even a whole trip might be left unrecorded. Subjects are not willing to wait for the device to start up at the beginning of every trip. In places surrounded by relatively tall building, the satellite signal reception of the two GPS units is affected. The devices may not record data or record incorrect data resulting in position errors or no position reading in some instances.

Another common problem observed during analysis of the data obtained from experiments using the two GPS units was data obtained for elevations. Out of 4 trips analyzed, in detail, 2 trips i.e. 50\%, showed elevation profiles (profiles obtained by plotting elevation data against trip distance) that did not reflect the actual topographic conditions at those locations. Thus, elevation data may not be appropriate or reliable.

The two GPS units provided accurate data on the position of the user. On this basis, a very good idea of the origins and destinations and the route selected by the user for a trip can be obtained. In addition to origins and destinations and route, the recorded data also provides information on speeds and delays.

Besides the above mentioned characteristics, a number of findings were made with respect to operational characteristics specific for each GPS device. For the handheld GPS, the data obtained from the experiments, sometimes showed inconsistencies in more than the afore-mentioned elevations, such as speeds. The reason for inconsistency in speeds can be assumed to be due to lack of satellite signals. These inconsistencies may pose a problem when results are used for engineering and/or planning purposes. 
While the hand-held unit is small and light-weight, and was not uncomfortable or bothersome to carry, the device may interfere with activities of the user since the GPS unit cannot be covered by any clothing or kept in a pocket. Another concern is that the unit must be held upright for it to communicate properly with the satellites.

The Wearable GPS data-logger gives a detailed record of the outdoor travel history of the device user. Since the trip information can be recorded after every 5 seconds (versus 15-30 seconds for the hand-held), delays enroute are much easier to determine. The device also provides information on the number of satellites to which it is connected.

One significant drawback of the device is its size, which interferes with user movements or activities. Users may feel uncomfortable carrying the GPS unit on their shoulder. The device may also be too conspicuous for users since the receiver antenna, attached to the shoulder strap, cannot be covered by any clothing. The cost of the wearable GPS data-logger also makes it less feasible to use in cases where large sample sizes are needed.

\section{Overall Conclusions}

On the basis of the results of the evaluations described herein, overall conclusions regarding the feasibility of each device were reached. These are presented below.

\section{Pedometer}

Since it provides information on step count only and lacks data storage capability, it was concluded that the pedometer is not feasible for use as a pedestrian travel data collection device. 


\section{$\underline{\text { Hand-Held GPS Unit }}$}

The assessment concluded that hand-held GPS device can be used to collect pedestrian travel data, such as positions, speeds, origins and destinations and route selection, with certain restrictions and under specific conditions. These restrictions involve various field conditions where the data collected may not prove to be fully reliable. These restrictions and conditions are:

- The unit should not be used in the vicinity of tall buildings and topography with high relief.

- The unit should not be used under thick forest cover or under dense tree canopies.

- The initial data will be inaccurate and unreliable if the user does not wait in one place for the initial two-minute device start-up time to allow it to communicate with the satellites.

- Data from the unit should be used for planning purposes only, not for detailed design.

\section{$\underline{\text { Wearable GPS Data-logger }}$}

A key conclusion of the evaluation described in this document is that it is feasible to use the Wearable GPS data logger for collecting pedestrian travel data, such as positions, speeds, origins and destinations, date and time information for trips and route selection, with certain restrictions and under specific conditions. These restrictions involve various field conditions where the data collected may not prove to be fully reliable. These restrictions and conditions:

- The unit should not be used in the vicinity of tall buildings and topography with high relief. 
- The initial data will be inaccurate and unreliable, if the user does not wait in place for the two-minute device start-up time to allow it to communicate with the satellites.

- It may be more feasible to use this unit in cases where the sample size is small as the cost of the unit is high.

- Data from the unit should be used for planning purposes only, not for detailed design. Finally, although the hand-held GPS records less detailed information than the wearable GPS data-logger, it may prove to a better option and more feasible to collect data on a larger scale as it almost one-tenth the price of the wearable data-logger. In addition, the hand-held GPS unit is smaller and easier to carry in comparison to the wearable unit but must be held upright. The cost and the size of the data-logger are noteworthy drawbacks even though it is a more accurate data collection device. Thus, it may be more useful and feasible for studies where the sample size is small.

\subsection{Recommendations}

The conclusions presented above were based on experiments conducted by the researcher as part of an unfunded research study. Thus, the number of subjects and experimental sites were somewhat limited. Additional work in this area is recommended as discussed here. This section is divided in two parts. The first part discusses recommendations specifically for practitioners and talks about issues that need to be considered while collecting data using the devices discussed in this study. The second part discusses recommendations for research extending or supporting the current work. Since it was concluded that the pedometer is not a feasible device for collecting pedestrian travel data, no recommendations for its use are included. 


\section{Recommendations for Practitioners}

This section discusses some of the recommendations that would be helpful to practitioners or professionals who may use the methods or devices discussed in this study, to collect pedestrian travel data. The recommendations give general guidance on when and how to use each device. The initial recommendations are common for both GPS devices and are followed by recommendations for practitioners specific to each device.

\section{General Recommendations for the two GPS Devices}

- The "Effect of Tall Buildings on Satellite Signal" experiment showed that the GPS units worked well in recording data when the device was between buildings on the order of 2 to 3 stories tall. As the building height increased and spacing between buildings decreased, acquiring satellite signals and, hence, recording data became more difficult. Thus, it is recommended that practitioners not use the GPS devices in the vicinity of closely spaced tall buildings as satellite signal availability is adversely affected.

- The experiment to assess operational considerations showed that the elevations recorded by both units may not be reliable. Thus, it is recommended that practitioners not use the elevation data acquired from the two GPS units.

- The experiments performed using the two GPS units gave sufficiently accurate data on the position of the user. On this basis, a very good idea of the origins and destinations and the route selected by the user for a trip can be obtained. In addition, the recorded data also provides information on speeds and delays. Thus, it is 
recommended that practitioners use the two GPS units for studies which require the above mentioned travel data.

- The experiments showed that the GPS units may lose communication with satellites for a period of time under dense tree canopy and in vicinity of high relief terrain. This loss of signal may affect the data recording capabilities of the units, and some points along a route may not be recorded. Thus, it is recommended that practitioners collecting pedestrian data should avoid using the GPS units under these conditions until additional research can be done to better define the limitations.

\section{Hand-Held GPS Unit}

- The experiment to assess the operation of the hand-held GPS unit showed that the correct way to hold the GPS unit was upright and that the unit may lose satellite signal if the unit is not held upright. Thus, it is recommended that practitioners either instruct the subject on the correct way to hold the GPS unit so as not to lose the satellite signal or provide them with a holster that will facilitate correct orientation of the device.

- Since the cost of the Hand-held GPS unit is not very high, it is recommended that practitioners consider this device for gathering pedestrian travel information in studies when cost is an issue.

- It is also recommended that the hand-held GPS unit be used in situations where track information is not required to be recorded every few seconds.

\section{$\underline{\text { Wearable GPS Data-Logger Unit }}$}

- The use of wearable GPS data-logger is recommended for studies where track information is required every few seconds. 
- The wearable GPS data-logger, in comparison with the hand-held GPS unit, gives more accurate data in terms of positions, speeds and route selected since it consists of a more sensitive antenna. Thus, its use is recommended for studies where more detailed and more accurate data are required. For example, the wearable GPS datalogger can be used in studies where pedestrian delays along a route are required.

\section{Recommendations for Researchers}

This section discusses recommendations for researchers. The recommendations mention some of the areas where this study can be improved and topics where further research may be required.

After conducting the experiments to assess the device characteristics of the handheld GPS unit and the wearable GPS data-logger, the following recommendations were identified.

- Only one type of high-voltage power line was considered; the effect may vary with the different types or configurations of power lines. Also, the distance between the power line and the GPS devices remained essentially constant and thus the effect of distance was not considered. Thus, additional research is recommended to study the effects of other types of power lines, different voltages and varying distances to the power lines on the satellite signal availability.

- The experiment to assess the effect of dense tree cover was performed for only two types of tree cover. Thus, further research would be desirable to study the effect under different types of tree cover, such as under a dense forest or particularly dense species. 
- Additional research is recommended to ascertain the effect of terrain with high relief on the availability of satellite signal, since these factors may play an important role in affecting the data recorded by the two GPS units.

- The experiment to assess the effect of rainfall on satellite signal, involved only a single run under one particular (and unquantified) rainfall intensity. It cannot be concluded that the GPS units would perform equally well in even heavier rains or other weather circumstances such as heavy snow and thick fog. Thus, further research is recommended in performing more rigorous experiments in varying weather conditions to ascertain their effect on the utility of devices.

- Additional research is also recommended to study the interaction effect of field conditions such as effect of high-voltage power lines along with heavy precipitation on the signal availability, or under trees covered with heavy snow.

The implementation or practical use of procedures described in this study is encouraged. Further research is recommended for evaluating other technologically advanced devices which are non-intrusive, low-cost and user-friendly and can be used to collect pedestrian data. 


\section{LIST OF REFERENCES}

Adamu, Ayalew, "GPS for Travel Surveys: Innovation in Household Travel Surveys", California Transportation Journal, Volume 3, Issue 4, January-March 2003, pp. 23-25. http://www.dot.ca.gov/dist07/news/publications/journal/janmar03.pdf

Aerospace Corporation, GPS Primer A Student Guide to the Global Positioning System, 2003.

http://www.aero.org/education/primers/gps/GPS-Primer.pdf

Axhausen, K.W., Travel Diaries: An Annotated Catalogue, $2^{\text {nd }}$ Edition, Institut fur Strassenbau und Verkehrsplanung, June 1995.

http://ntl.bts.gov/lib/6000/6900/6973/td.pdf

Bassett, David R. Jr., Ainsworth, Barbara E., Leggett, Sue R., Mathien, Clara A., Main, James A., Hunter, David C. and Duncan, Glen E., "Accuracy of Five Electronic Pedometers for Measuring Distance Walked", Medicine and Science in Sports and Exercise, Vol. 28, No. 8, 1996, pp. 1071-1077.

Bullock, Darcy; Quiroga, Cesar and Schwehm, Chris, A GPS Methodology for Conducting Travel Time Studies, Louisiana Transportation Research Center, LSU Remote Sensing and Image Sensing Laboratory, 1998

http://www.rsip.lsu.edu/projects/cms/cmshome.html

Chang, Kang Tsung, Introduction to Geographic Information Systems, $1^{\text {st }}$ Edition, McGraw Hill, USA, pp. 3, September 2001.

Consumers Union of the United States, Inc. "Walking by the Numbers", Consumer Reports, October 2004, pp. 30-31.

Dishman, Rod K., Washburn, Richard A. and Schoeller, Dale A., "Measurement of Physical Activity", Quest, Volume 53, Issue No. 3, August 2001, pp. 295-309.

Draper, Stephen W., "The Hawthorne, Pygmalion, Placebo and Other Effects of Expectation: Some Notes", Department of Psychology, University of Glasgow, May 2002.

http://www.psy.gla.ac.uk/ steve/hawth.html

ESRI, GIS Dictionary

http://support.esri.com/index.cfm?fa=knowledgebase.gisDictionary.search\&searchTerm= GIS

Freedson, P.S. and Miller, K., "Objective Monitoring of Physical Activity Using Motion Sensors and Heart Rate", Research Quarterly for Exercise and Sport, Volume 71, No. 2, pp. 21-29, 2000. 
Garber, Nicholas. J., and Hoel, Lester. A., Traffic and Highway Engineering, Third Edition, Thomson Learning, USA, 2002, pp. 117-121.

Gasvoda, Dave, “Trail Traffic Counters: Update”, U.S. Department of Agriculture Forest Service, Technology and Development Program, September 1999, http://www.fhwa.dot.gov/environment/Fspubs/99232835/index.htm

De Jong, Robert and Mensonides, Wytse, "Wearable GPS Device as a Data Collection Method for Travel Research", Working Paper ITS-WP-03-02, ISSN 1440-3501, Institute of Transport Studies, Australia, February 2003.

Koike, Hirotaka, Morimoto, Akinori, Inoue, Tadashi and Kawano, Tomohiko, "Studies on the Characteristic Differences of Pedestrian Behaviors among Cities and between City Districts such as Downtown and Suburban Shopping Centers", The Fourth International Conference on Walking in the 21st Century, Portland, OR, USA, 2003

http://www.americawalks.org/walk21/pdf_key.htm

Landry, Shawn M. and Oliver, Naomi, M., "Rapid Completion of a GPS-Based Infrastructure Inventory for NPDES, GASB 34 and FEMA", Stormwater, The Journal for Surface Water Quality Professionals, Volume 3, No. 1, January-February 2002.

http://www.gradingandexcavation.com/sw_0201_rapid.html

Pierce, Sean and Lappin, Jane, "Acquisition of Traveler Information and Its Effects on Travel Choices: Evidence from a Seattle-Area Travel Diary Survey", Volpe National Transportation Systems Center, Cambridge, MA, April 2003.

http://www.itsdocs.fhwa.dot.gov/JPODOCS/REPTS_TE//13813.html

Roess, R. P., McShane, W. R., and Prassas, E. S., Traffic Engineering, Third Edition, Prentice Hall, Englewood Cliffs, NJ, USA, 1990.

Sequeira, Maria M., Rickenbach, Martin, Wietlisbach, Vincent, Tullen, Benoit and Schutz, Yves, "Physical Activity Assessment Using a Pedometer and Its Comparison with a Questionnaire in a Large Population Survey", American Journal of Epidemiology, Volume 142, No.9, 1995, pp. 989-999.

SRF Consulting Group, Inc., Bicycle and Pedestrian Detection: Final Report, Office of Traffic Engineering/ITS, Minnesota Department of Transportation, Minneapolis, MN, USA, February 2003, http://ntl.bts.gov/lib/23000/23300/23330/BikePedDetFinalReport.pdf

Stopher, Peter, Bullock, Philip and Horst, Frederic, "Exploring the Use of Passive GPS Devices to Measure Travel", Working Paper ITS-WP-02-06, ISSN 1440-3501, Institute of Transport Studies, Australia, January 2002a.

Stopher, Peter, Bullock, Philip and Jiang, Qingjian, "GPS, GIS and Personal Travel Surveys: An Exercise in Visualisation", $25^{\text {th }}$ Australasian Transport Research Forum- 
Incorporating the BTRE Transport Policy Colloquium, Canberra, Australia, October $2002 \mathrm{~b}$.

TRB, Highway Capacity Manual, National Research Council, Washington, DC, 2000

TRB Special Report 277, "Measuring Personal Travel and Goods Movement: A Review of the Bureau of Transportation Statistics' Surveys", National Research Council of the National Academies, 2004, pp.35-73.

TRB Special Report 282, "Does the Built Environment Influence Physical Activity? Examining the Evidence", Institute of Medicine of the National Academies, 2005, pp.138.

Trimble Navigation Limited, Mapping Systems General Reference, Part Number 2417700, Revision C, January 2000.

Tudor-Locke, Catrine, "Taking Steps Toward Increased Physical Activity: Using Pedometers to Measure and Motivate", Research Digest, President's Council on Physical Fitness and Sports, Series 3, No.17, June 2002, pp. 1-8.

http://www.fitness.gov/pcpfsdigestjune2002.pdf

Tudor-Locke, Catrine and Myers, Alan M., "Methodological Considerations for Researchers and Practitioners Using Pedometers to Measure Physical (Ambulatory) Activity", Research Quarterly for Exercise and Sport, Volume 72, No. 1, pp. 1-12, 2001.

U.S. Department of Transportation, "Bicycle and Pedestrian Data: Sources, Needs, and Gaps", Bureau of Transportation Statistics, Washington, DC, USA, 2000 http://www.bts.gov/publications/bicycle_and_pedestrian_data/entire.pdf

Welk, Gregory J., Differding, Jerome A., Thompson, Raymond W., Blair, Steven N., Dzuira, Jim and Hart, Peter, "The Utility of the Digi-Walker Step Counter to Assess Daily Physical Activity Patterns", Medicine and Science in Sports and Exercise, Vol. 32, No. 9, Suppl., 2000, pp. S481-S488.

Wolf, Jean, Guensler, Randall and Bachman, William, "Elimination of the Travel DiaryExperiment to Derive Trip Purpose from Global Positioning System Travel Data", Transportation Research Record 1768, 2001, pp. 125-134. 
APPENDIX A

Table of the Standard Normal (z) Distribution 
Table of the Standard Normal (z) Distribution

\begin{tabular}{|c|c|c|c|c|c|c|c|c|c|c|}
\hline Z & .00 & 0.01 & 0.02 & 0.03 & 0.04 & 0.05 & 0.06 & 0.07 & 0.08 & 0.09 \\
\hline 0.0 & 000 & 0.0040 & 0.0080 & 0.0120 & 0.0160 & 0.0190 & 0.0239 & 0.0279 & 0.0319 & 0.0359 \\
\hline & 398 & 0438 & 0.0478 & 0.0517 & 0.0557 & 0.0596 & 0.0636 & 0.0675 & 0.0714 & 0.0753 \\
\hline 0.2 & 0793 & .0832 & 0.0871 & 0.0910 & 0.0948 & 0.0987 & 0.1026 & 0.1064 & 0.1103 & 0.1141 \\
\hline & 1179 & 1217 & 0.1255 & 0.1293 & 0.1331 & 0.1368 & 0.1406 & 0.1443 & 0.1480 & 0.1517 \\
\hline 0.4 & 1554 & 0.1591 & 0.1628 & 0.1664 & 0.1700 & 0.1736 & 0.1772 & 0.1808 & 0.1844 & 0.1879 \\
\hline 5 & 1915 & 0.1950 & 0.1985 & 0.2019 & 0.2054 & 0.2088 & 0.2123 & 0.2157 & 0.2190 & 0.2224 \\
\hline 0.6 & 2257 & 0.2291 & 0.2324 & 0.2357 & 0.2389 & 0.2422 & 0.2454 & 0.2486 & 0.2517 & 0.2549 \\
\hline 0.7 & 0.2580 & 0.2611 & 0.2642 & 0.2673 & 0.2704 & 0.2734 & 0.2764 & 0.2794 & 0.2823 & 0.2852 \\
\hline & 2881 & 0.2910 & 0.2939 & 0.2969 & 0.2995 & 0.3023 & 0.3051 & 0.3078 & 0.3106 & 0.3133 \\
\hline 0.9 & 0.3159 & 0.3186 & 0.3212 & 0.3238 & 0.3264 & 0.3289 & 0.3315 & 0.3340 & 0.3365 & 0.3389 \\
\hline & 3413 & 3438 & 0.3461 & 0.3485 & 0.3508 & 0.3513 & 0.3554 & 0.3577 & 0.3529 & 0.3621 \\
\hline 1.1 & 0.3643 & 0.3665 & 0.3686 & 0.3708 & 0.3729 & 0.3749 & 0.3770 & 0.3790 & 0.3810 & 0.3830 \\
\hline & 3849 & 0.3869 & 0.3888 & 0.3907 & 0.3925 & 0.3944 & 0.3962 & 0.3980 & 0.3997 & 0.4015 \\
\hline 1.3 & 0.4032 & 0.4049 & 0.4066 & 0.4082 & 0.4099 & 0.4115 & 0.4131 & 0.4147 & 0.4162 & 0.4177 \\
\hline 1.2 & 0.4192 & 0.4207 & 0.4222 & 0.4236 & 0.4251 & 0.4265 & 0.4279 & 0.4292 & 0.4306 & 0.4319 \\
\hline 1.5 & 0.4332 & 0.4345 & 0.4357 & 0.4370 & 0.4382 & 0.4394 & 0.4406 & 0.4418 & 0.4429 & 0.4441 \\
\hline 1.6 & 0.4452 & 0.4463 & 0.4474 & 0.4484 & 0.4495 & 0.4505 & 0.4515 & 0.4525 & 0.4535 & 0.4545 \\
\hline 1.7 & 0.4554 & 0.4564 & 0.4573 & 0.4582 & 0.4591 & 0.4599 & 0.4608 & 0.4616 & 0.4625 & 0.4633 \\
\hline 1.8 & 0.4641 & 0.4649 & 0.4656 & 0.4664 & 0.4671 & 0.4678 & 0.4686 & 0.4693 & 0.4699 & 0.4706 \\
\hline 1.9 & 0.4713 & 0.4719 & 0.4726 & 0.4732 & 0.4738 & 0.4744 & 0.4750 & 0.4756 & 0.4761 & 0.4767 \\
\hline 2.0 & 0.4772 & 0.4778 & 0.4783 & 0.4788 & 0.4793 & 0.4798 & 0.4803 & 0.4808 & 0.4812 & 0.4817 \\
\hline 2.1 & 0.4821 & 0.4826 & 0.4830 & 0.4834 & 0.4838 & 0.4842 & 0.4846 & 0.4850 & 0.4854 & 0.4857 \\
\hline 2.2 & 0.4861 & 0.4864 & 0.4868 & 0.4871 & 0.4875 & 0.4878 & 0.4881 & 0.4884 & 0.4887 & 0.4890 \\
\hline 2.3 & 0.4893 & 0.4896 & 0.4898 & 0.4901 & 0.4904 & 0.4906 & 0.4909 & 0.4911 & 0.4913 & 0.4916 \\
\hline 2.4 & 0.4918 & 0.4920 & 0.4922 & 0.4925 & 0.4927 & 0.4929 & 0.4931 & 0.4932 & 0.4934 & 0.4936 \\
\hline 2.5 & 0.4938 & 0.4940 & 0.4941 & 0.4943 & 0.4945 & 0.4946 & 0.4948 & 0.4949 & 0.4951 & 0.4952 \\
\hline 2.6 & 0.4953 & 0.4955 & 0.4956 & 0.4957 & 0.4959 & 0.4960 & 0.4961 & 0.4962 & 0.4963 & 0.4964 \\
\hline 2.7 & 0.4965 & 0.4966 & 0.4967 & 0.4968 & 0.4969 & 0.4970 & 0.4971 & 0.4972 & 0.4973 & 0.4974 \\
\hline 2.8 & 0.4974 & 0.4975 & 0.4976 & 0.4977 & 0.4977 & 0.4978 & 0.4979 & 0.4979 & 0.4980 & 0.4981 \\
\hline 2.9 & 0.4981 & 0.4982 & 0.4982 & 0.4983 & 0.4984 & 0.4984 & 0.4985 & 0.4985 & 0.4986 & 0.4986 \\
\hline 3.0 & 0.4987 & 0.4987 & 0.4987 & 0.4988 & 0.4988 & 0.4989 & 0.4989 & 0.4989 & 0.4990 & 0.4990 \\
\hline 3.1 & 0.4990 & 0.4991 & 0.4991 & 0.4991 & 0.4992 & 0.4992 & 0.4992 & 0.4992 & 0.4993 & 0.4993 \\
\hline 3.2 & 0.4993 & 0.4993 & 0.4994 & 0.4994 & 0.4994 & 0.4994 & 0.4994 & 0.4995 & 0.4995 & 0.4995 \\
\hline 3.3 & 0.4995 & 0.4995 & 0.4995 & 0.4996 & 0.4996 & 0.4996 & 0.4996 & 0.4996 & 0.4996 & 0.4997 \\
\hline 3.4 & 0.4997 & 0.4997 & 0.4997 & 0.4997 & 0.4997 & 0.4997 & 0.4997 & 0.4997 & 0.4997 & 0.4998 \\
\hline
\end{tabular}

The values inside the given table represent the areas under the standard normal curve for values between 0 and the relative z-value.

(Source-http://www.isixsigma.com/library/content/zdistribution.asp) 


\section{APPENDIX B}

\section{Instruction Sheets and Data Collection Forms}

For Experiments to Evaluate Device Characteristics 


\section{Instruction Sheet for \\ Effect of Surface Type on Pedometer Steps}

\section{$\underline{\text { Background }}$}

This experiment involves determining the feasibility of using various 'wearable' devices for collecting data about pedestrian characteristics. One of the devices being studied is a pedometer, a device that basically counts steps. This device is going to be used in our experiments. These experiments are being conducted to determine the effect of different types of surfaces on the number of steps needed to cover a given distance. The step count will be made using the pedometer.

The identity of the subjects and the data collected about the subjects will be kept confidential and will be known only to the researcher. The subjects will be given a code number and any references to an individual subject will be made by their respective code number.

\section{Getting Ready for the Experiment}

In order to successfully complete the pedometer experiments, subjects will need to comply with the following:

1. Please wear sport shoes with rubber soles. Leather-sole shoes, sandals, shoes with heels, boots and slippers may affect the walking stride and cannot be allowed.

2. While doing the experiment, the subject should not be carrying large back-packs, books, hand-bags, umbrellas, laptop cases, large purses or any other significant objects as these can affect the manner of walking. 
3. The subjects are expected to wear pants, trousers or jeans, but no skirts and shorts. Only waist-length sweaters, jackets and other attire should be worn. Long overcoats, parkas, raincoats or long dresses are not appropriate.

4. To minimize the effects of external factors, subjects are not permitted to eat, drink, smoke or use cell-phones during the experiment.

\section{Experimental Procedure}

We will first determine your stride length by asking you to walk a distance of 60 feet and record the number of steps. This will be done twice and the average will be calculated. After your stride length has been recorded, you will walk a distance of 300 feet, twice (out and back) on each of the surface specified. Before starting each experiment, you will be given a pedometer which is to be worn on the waist (approximately in the center of the right leg). At the start point of each run, the pedometer will be set to zero.

You should then walk at your normal pace through the study section. Try to maintain a constant pace and walk in a straight line. You should avoid speaking to anyone (except in case of emergency) and try to focus on the task at hand. Do not talk on the cell-phone, smoke, eat or drink while walking. As soon as you cross the finish line, stop and the researcher will record the pedometer reading, and reset it to zero for the second test. The second test will be on the same section of walkway but in the opposite direction. The same procedure and rules apply. 


\section{Data Sheet for}

\section{Effect of Surface Type on Steps}

Subject:

Date:

Recorder:

Length (test section):

Weather:

Pedometer: Yamax Digi-Walker
Stride Length of Subject:

Gender of Subject: M / F

Height of Subject:

Type of Shoes:

Observations:

\begin{tabular}{|l|c|c|c|c|c|c|c|c|c|c|c|c|}
\hline $\begin{array}{l}\text { Surface } \\
\text { Type }\end{array}$ & \multicolumn{3}{|c|}{ High-Type } & \multicolumn{4}{c|}{ Grass } & \multicolumn{3}{c|}{ Unpaved } & \multicolumn{3}{c|}{$\begin{array}{c}\text { Older } \\
\text { Deteriorated }\end{array}$} \\
\hline & Rep1 & Rep2 & Avg. & Rep1 & Rep2 & Avg. & Rep1 & Rep2 & Avg. & Rep1 & Rep2 & Avg. \\
\hline $\begin{array}{l}\text { Step } \\
\text { Count }\end{array}$ & & & & & & & & & & & & \\
\hline
\end{tabular}

Comments: 


\section{Instruction Sheet for \\ Effect of Gradient on Pedometer Steps}

\section{$\underline{\text { Background }}$}

This experiment involves determining the feasibility of using various 'wearable' devices for collecting data about pedestrian characteristics. One of the devices being studied is a pedometer, a device that basically counts steps. This device is going to be used in our experiments. These experiments are being conducted to determine the effect of walkway gradient on the number of steps needed to cover a given distance. The step count will be made using the pedometer.

The identity of the subjects and the data collected about the subjects will be kept confidential and will be known only to the researcher. The subjects will be given a code number and any references to an individual subject will be made by their respective code number.

\section{Getting Ready for the Experiment}

In order to successfully complete the pedometer experiments, subjects will need to comply with the following:

1. Please wear sport shoes with rubber soles. Leather-sole shoes, sandals, shoes with heels, boots and slippers may affect the walking stride and cannot be allowed.

2. While doing the experiment, the subject should not be carrying large back-packs, books, hand-bags, umbrellas, laptop cases, large purses or any other significant objects as these can affect the manner of walking. 
3. The subjects are expected to wear pants, trousers or jeans, but no skirts and shorts. Only waist-length sweaters, jackets and other attire should be worn. Long overcoats, parkas, raincoats or long dresses are not appropriate.

4. To minimize the effects of external factors, subjects are not permitted to eat, smoke drink, or use cell-phones during the experiment.

\section{Experimental Procedure}

We will first determine your stride length by asking you to walk a distance of 60 feet and record the number of steps. This will be done twice and the average will be calculated. After your stride length has been recorded, you will walk a distance of 200 feet, twice (upgrade and downgrade) on each of the gradient specified. Before starting each experiment, you will be given a pedometer which is to be worn on the waist (approximately in the center of the right leg). At the start point of each run, the pedometer will be set to zero.

You should then walk at your normal pace through the study section. Try to maintain a constant pace and walk in a straight line. You should avoid speaking to anyone (except in case of emergency) and try to focus on the task at hand. Do not talk on the cell-phone, smoke, eat or drink while walking. As soon as you cross the finish line, stop and the researcher will record the pedometer reading, and reset it to zero for the second test. The second test will be on the same section of walkway but in the opposite direction. The same procedure and rules apply. 


\section{Data Sheet for}

\section{Effect of Gradient on Steps}

Subject:

Date:

Recorder:

Length (test section):

Weather:

Pedometer: Yamax Digi-Walker
Stride Length of Subject:

Gender of Subject: M / F

Height of Subject:

Type of Shoes:

Observations:

\begin{tabular}{|l|c|l|l|l|l|l|l|l|}
\hline Gradient & \multicolumn{2}{|c|}{$0-2 \%$} & \multicolumn{2}{c|}{$3-7 \%$} & \multicolumn{2}{c|}{$8-12 \%$} & \multicolumn{2}{c|}{$13-17 \%$} \\
\hline & Upgrade & Downgrade & Upgrade & Downgrade & Upgrade & Downgrade & Upgrade & Downgrade \\
\hline $\begin{array}{l}\text { Step } \\
\text { Count }\end{array}$ & & & & & & & & \\
\hline
\end{tabular}

Comments: 


\section{Instruction Sheet for}

\section{Effect of Social Interaction on Pedometer Steps}

\section{$\underline{\text { Background }}$}

This experiment involves determining the feasibility of using various 'wearable' devices for collecting data about pedestrian characteristics. One of the devices being studied is a pedometer, a device that basically counts steps. This device is going to be used in our experiments. The experiment is being conducted to determine the effect of social interaction on number of steps. The step count will be made using the pedometer.

The identity of the subjects and the data collected about the subjects will be kept confidential and will be known only to the researcher. The subjects will be given a code number and any references to an individual subject will be made by their respective code number.

\section{Getting Ready for the Experiment}

In order to successfully complete the pedometer experiments, subjects will need to comply with the following:

1. Please wear sport shoes with rubber soles. Leather-sole shoes, sandals, shoes with heels, boots and slippers may affect the walking stride and cannot be allowed.

2. While doing the experiment, the subject should not be carrying large back-packs, books, hand-bags, umbrellas, laptop cases, large purses or any other significant objects as these can affect the manner of walking. 
3. The subjects are expected to wear pants, trousers or jeans, but no skirts and shorts. Only waist-length sweaters, jackets and other attire should be worn. Long overcoats, parkas, raincoats or long dresses are not appropriate.

4. To minimize the effects of external factors, subjects are not permitted to eat, smoke, drink, or use cell-phones during the experiment.

\section{Experimental Procedure}

We will first determine your stride length by recording the number of steps required for you to walk a distance of 200 feet. After your stride length has been recorded, you will walk the length of test-section (200 feet), three times with the researcher, under different test conditions as specified. Since the effect of social interaction is to be determined, there will be three levels of interaction (trials) between the researcher and the subject. During the first trial, the researcher will walk side-by-side with you at a comfortable separation. There should be no conversation or eye-contact between you and the researcher, and both should walk facing straight ahead. In the next trial, you will again walk with the researcher, but this time there will be limited talking. Limited talking refers to short conversations (for example, questions asked by the researcher that can be answered with a yes or no, or merely a short reply). This is intended to be a two-way conversation. The conversation will be initiated by the researcher, so wait for his cue. In the third and final trial, you will again walk with the researcher; and this time there will be significant conversation throughout the walk. Significant conversation here refers to a two-way conversation in which both researcher and subject are almost equally participating, and is lengthier than the short conversation, i.e. more than short replies. For example, the researcher may ask questions or talk about 
current events, educational topics, sports or other similar topics of discussion. You may ask questions or talk about the same or related issues.

During the trials, you should walk at your normal pace through the study section. As soon as you cross the finish line, you should stop and get ready for the next run after a two minute rest. The same rules will apply. 


\section{Data Sheet for}

\section{Effect of Social Interaction on Steps}

\section{Subject}

Date:

Length (test section):

Weather:

Pedometer: Yamax Digi-Walker

Stride Length of Researcher:

(Length of test section)/ Researcher's steps when walking alone

Stride Length of Accompanying Subject:

(Length of test section)/ Subject's steps when walking alone

Height of Subject:

Gender of Subject: $\mathrm{M} / \mathrm{F}$

Observations:

\begin{tabular}{|l|l|l|}
\hline \multicolumn{1}{|c|}{ Test Condition } & Steps & Comments \\
\hline Researcher walking alone & & \\
\hline Subject walking alone & & \\
\hline Researcher with subject - No Talking & & \\
\hline Researcher with subject - Limited Talking & & \\
\hline Researcher with subject - Significant Conversation & & \\
\hline
\end{tabular}

Comments: 


\section{Data Sheet for}

\section{Effect of Other Pedestrian Traffic on Steps}

Date:

Location of Test section:

Weather:

Pedometer: Yamax Digi-Walker

Stride Length of Researcher: 2.4 feet
Time:

Length of test section:

Width of test section:

\begin{tabular}{|c|c|}
\hline Test Run & Number of Steps \\
\hline 1 & \\
\hline 2 & \\
\hline 3 & \\
\hline 4 & \\
\hline 5 & \\
\hline 6 & \\
\hline 7 & \\
\hline 8 & \\
\hline 9 & \\
\hline 10 & \\
\hline 11 & \\
\hline 12 & \\
\hline 13 & \\
\hline
\end{tabular}




\section{Post-Processing Data Sheet for}

\section{Effect of Other Pedestrian Traffic on Steps}

Site No.

Date:

Length of test-section:

Width of test-section:

Determination of Pedestrian LOS from Field Data

\begin{tabular}{|c|c|c|c|c|c|}
\hline Test Run & Photo Number & $\begin{array}{l}\text { Number of } \\
\text { Pedestrians }\end{array}$ & Area of Section & $\begin{array}{c}\text { Pedestrian Space } \\
\text { (sq.ft./ped.) }\end{array}$ & Level of Service \\
\hline & & & & & \\
\hline & & & & & \\
\hline & & & & & \\
\hline & & & & & \\
\hline & & & & & \\
\hline & & & & & \\
\hline & & & & & \\
\hline & & & & & \\
\hline & & & & & \\
\hline & & & & & \\
\hline & & & & & \\
\hline & & & & & \\
\hline & & & & & \\
\hline
\end{tabular}




\section{Data Sheet for}

\section{Signal Availability Under High-Voltage Power Lines and Towers}

Date:

Site:

Type of Tower:

Weather:

Recorder:

Comments:

Observations:

\section{Hand-Held GPS unit}

\begin{tabular}{|l|l|l|l|}
\hline Clock time & Location & $\begin{array}{c}\text { No. of satellite signals } \\
\text { on display screen }\end{array}$ & Accuracy in ft. \\
\hline & & & \\
\hline & & & \\
\hline & & & \\
\hline & & & \\
\hline
\end{tabular}

Findings: 


\section{Data Sheet for}

\section{Signal Availability Under Dense Tree Canopy}

Date:

Location:

Type of Vegetation: Deciduous / Coniferous

Weather:

Recorder:

Comments:

Observations:

\section{Hand-Held GPS unit}

\begin{tabular}{|l|l|l|}
\hline Clock time & $\begin{array}{c}\text { No. of satellite signals } \\
\text { on display screen }\end{array}$ & Accuracy in $\mathrm{ft.}$ \\
\hline & & \\
\hline & & \\
\hline & & \\
\hline & & \\
\hline
\end{tabular}

Findings: 


\section{Data Sheet for}

\section{Signal Availability in Heavy Rain}

Date:

Location:

Weather:

Precipitation Intensity/ Details:

Recorder:

Comments:

Observations:

\section{Hand-Held GPS unit}

\begin{tabular}{|l|l|l|}
\hline Clock time & $\begin{array}{c}\text { No. of satellite signals } \\
\text { on display screen }\end{array}$ & Accuracy in ft. \\
\hline & & \\
\hline & & \\
\hline & & \\
\hline & & \\
\hline
\end{tabular}

Findings: 


\section{Data Sheet for}

\section{Signal Availability in Vicinity of Tall Buildings}

Date:

Location:

Number of Stories in Surrounding Buildings:

Spatial Separation:

Weather:

Recorder:

Comments:

\section{Observations:}

\section{Hand-Held GPS unit}

\begin{tabular}{|l|l|l|}
\hline Clock time & $\begin{array}{c}\text { No. of satellite signals } \\
\text { on display screen }\end{array}$ & Accuracy in ft. \\
\hline & & \\
\hline & & \\
\hline & & \\
\hline & & \\
\hline
\end{tabular}

Findings: 


\begin{abstract}
APPENDIX C
Instruction Sheets and Data Collection Forms for Experiments to Evaluate Operational Considerations
\end{abstract}




\section{Instruction Sheet for the}

\section{Use of Pedometer for Collecting Non-Motorized Travel Data}

\section{$\underline{\text { Background }}$}

This study involves determining the feasibility of using various 'wearable' devices for collecting data about pedestrian travel. One of the devices being studied is a pedometer, a device that counts the number of steps taken by the person wearing it. The device is currently being used as a motivator to increase physical activity in the general public. Since the pedometer is easy-to-use and is already relatively widespread in the population, it is of interest to determine the feasibility of using pedometers to acquire pedestrian travel data. The purpose of the pedometer is simply to count steps taken by the user to cover the distance from an origin to a destination. Along with the pedometer, the subjects will also be given a travel log form where they will record information about each trip. This will be done in order to know the duration of trips as well as the origins and destinations. The researcher will analyze the data to try to determine the feasibility of using the pedometer as a travel data collection tool.

The identity of the subjects and the data collected from each subject will be kept confidential and will be known only to the researcher. The subjects will be given a number and any references to an individual subject will be made by their respective number.

\section{Getting Ready for the Experiment}

In order to successfully complete the pedometer study, subjects will be expected to comply with the following: 
- The pedometer unit should be handled and operated carefully just as you would any electronic device.

- Each time the pedometer is worn, the subject should take care that the device is clipped on the waist at the center of the leg, as demonstrated.

- The subject should close the pedometer properly whenever in use, or there may be errors in recording number of steps.

- The travel log form should be completed at the time of each motorized or nonmotorized trip.

\section{Experimental Procedure}

This experiment is being conducted to determine the feasibility of using pedometers to collect pedestrian travel data. You will be given the pedometer around mid-day and asked to use it for a period of 24 hours. During this time, you should record all walking trips made out-of-doors. The pedometer will be worn on the waist at approximately the center of the right leg, as demonstrated. The pedometer should be worn for the entire duration of the experiment. Take care to re-attach the pedometer when you change clothes and at the beginning of the day. Whenever you leave a building to go to another destination by walking, you will need to reset the pedometer to zero, once you are outside. After resetting it to zero, the pedometer needs to be closed properly, after which you can walk to your destination. Take care that the pedometer is clipped securely and does not fall off while walking or while in a rest room. Once you have reached your destination, read and record the number of steps as shown on the pedometer display. This process should be carried out for all walking trips during the specified period. 
Along with the pedometer unit, you will also be given a travel log form which should be completed for all trips you make (including other modes such as car, PRT and bus). You should clearly identify the origin/destination information in terms of a descriptive name for identification. Also note the time of departure/arrival for each location along with the mode of travel used. Again, the travel log form will be completed for all non-motorized and motorized trips, while the pedometer unit will be reset to zero only for walking trips. In case of intermodal trips, for example, the subject walks half the distance and then catches a bus to the final destination, the subject should record the origin and destination information to the point where the mode changed. The number of steps at this change point should be recorded. For the second portion of the trip (the bus), the subject should record the 'origin' (as the point where the mode changed) and the destination information, without recording any step count.

At the end of the specified 24 hours, return the pedometer and completed travel $\log$ form to the researcher. You will then be asked to complete a brief questionnaire on your experience. 


\section{Pedometer Travel Log Form}

\begin{tabular}{|c|c|}
\hline SUBJECT--- & DATE--- \\
\hline Origin- & Origin- \\
\hline Origin time - & Origin time - \\
\hline Destination- & Destination- \\
\hline Destination time- & Destination time- \\
\hline Mode- & Mode- \\
\hline Origin- & Origin- \\
\hline Origin time - & Origin time - \\
\hline Destination- & Destination- \\
\hline Destination time- & Destination time- \\
\hline Mode- & Mode- \\
\hline Origin- & Origin- \\
\hline Origin time - & Origin time - \\
\hline Destination- & Destination- \\
\hline Destination time- & Destination time- \\
\hline Mode- & Mode- \\
\hline Origin- & Origin- \\
\hline Origin time - & Origin time - \\
\hline Destination- & Destination- \\
\hline Destination time- & Destination time- \\
\hline Mode- & Mode- \\
\hline Origin- & Origin- \\
\hline Origin time - & Origin time - \\
\hline Destination- & Destination- \\
\hline Destination time- & Destination time- \\
\hline Mode- & Mode- \\
\hline
\end{tabular}




\section{Subject Post-Test Questionnaire on Using Pedometers to Collect Non-Motorized \\ Travel Data}

\section{Subject}

Date:

Date of Study:

1. Did you ever fail to take the pedometer with you any on any walking trips? If yes, about how many times?

2. Did you ever fail to reset the pedometer to zero? If yes, about how many times?

3. Did you ever forget to take the travel log form with you when leaving a building? If yes, about how many times?

4. Did you ever fail to record certain information about a walking trip when you were wearing the pedometer? If yes, about how many times?

5. Did you ever fail to record an entire trip on the travel data form? If yes, how many times?

6. Did you think it was uncomfortable/bothersome to wear the pedometer everywhere for your trips outside?

7. Did you think it was bothersome to have to carry and record all trips made, on the travel $\log$ form?

8. Did you find it difficult to read the number of steps displayed on pedometer screen at the end of each trip?

9. Did the pedometer ever slip off your waist while walking? If yes, would you say it happened - rarely occasionally frequently ? 
10. Did you feel that pedometer affected your physical/personal appearance? How?

11. Did the pedometer interfere with any movements or activities? If yes, please explain

12. Does it bother you that someone knows about all of the walking trips you made during the period of the experiment? 


\title{
Instruction Sheet for the
}

\section{Use of Hand-Held GPS Device for Collecting Non-Motorized Travel Data}

\author{
$\underline{\text { Background }}$
}

This study involves determining the feasibility of using various 'wearable' devices for collecting data about pedestrian travel. One of the devices being studied is a hand-held GPS unit, a device that connects to the satellite system to accurately provide position, velocity and time information to those with access to a GPS-enabled device. This device is going to be used in our experiment. This experiment is being conducted to collect certain travel data such as route selected, trip length, travel speed, delays en route and origins and destinations.

Our interest in this research is in determining the feasibility of using GPS technology to acquire pedestrian travel data rather than in identifying the travel patterns of individuals. The identity of the subjects and the data collected from each subject will be kept confidential and will be known only to the researcher. The subjects will be given a number and any references to an individual subject will be made by their respective number.

\section{Getting Ready for the Experiment}

In order to successfully complete the hand-held GPS device study, subjects will be expected to comply with the following:

- The GPS unit should be handled and operated carefully just as you would any electronic device.

- The GPS device, after being switched on, should be carried in the upright vertical position, whether being carried in hand or in the holster. 
- The subject should switch on the GPS unit for all walking trips.

- The travel log form should be completed at the time of each motorized or nonmotorized trip.

\section{Experimental Procedure}

This experiment is being conducted to ascertain the feasibility of the hand-held GPS device for use as a pedestrian travel data collection device. You will be given the GPS unit at noon for a period of 24 hours. During this time, you should record all walking trips made out-of-doors. Whenever you leave a building to go for another destination by walking, you will need to switch on the GPS unit by pressing the power button located on the side of the unit, once you are outside. You will need to wait about 2 minutes at the start of each trip to allow the GPS device to determine your position, after which you can walk to your destination. The unit can be carried in the hand or kept in the holster provided. Take care that the GPS unit is placed/carried in the vertical upright position, as demonstrated by the researcher. Once you have reached your destination, you can switch off the GPS unit by pressing the power button, located on the side. This process should be carried out for all walking trips during the specified period.

Along with the GPS unit, you will also be given a travel log form which should be completed for all trips you make (including other modes such as car, PRT and bus). You should clearly identify the origin/destination information in terms of a descriptive name for identification. Also note the time of departure/arrival for each location along with the mode of travel used. Again, the travel log form will be completed for all non-motorized and motorized trips, while the GPS unit will be switched on only for walking trips outdoors. 
The next day, at the end of specified 24 hour period, return the GPS unit along with completed travel log form to the researcher. You will then be asked to complete a brief questionnaire on your experience. 


\title{
Subject Post-Test Questionnaire on Using Hand-Held GPS for Collecting Non-Motorized Travel Data
}

\author{
Subject
}

Date:

Date of Study:

1. Did you ever fail to take the hand-held GPS device with you any on any walking trips? If yes, about how many times?

2. Did you ever fail to switch on the GPS unit? If yes, about how many times?

3. Did you ever forget to wait the two minutes needed for the GPS unit to start-up? If yes, about how many times?

4. Did you ever forget to take the travel log form with you when leaving a building? If yes, about how many times?

5. Did you ever fail to record information about a walking trip? If yes, about how many times?

6. Did you ever fail to record an entire trip on the travel data form? If yes, how many times?

7. Did you think it was uncomfortable/bothersome to carry the GPS data-logger everywhere for your trips outside?

8. Did you think it was bothersome to have to carry and record all trips made, on the travel $\log$ form? 
9. Did the GPS unit interfere with any movements or activities? If yes, please explain

10. Does it bother you that someone knows about all of the walking trips you made during the period of the experiment? 


\section{Use of Wearable GPS Data-Logger Unit for Collecting Non-Motorized Travel Data}

\section{$\underline{\text { Background }}$}

This study involves determining the feasibility of using various 'wearable' devices for collecting data about pedestrian travel. One of the devices being studied is a wearable GPS data-logger, a device that connects to the satellite system to accurately provide position, velocity and time information to those with access to a GPS-enabled device. This experiment is being conducted to collect certain travel data such as route selected, trip length, travel speed, delays en route and origins and destinations.

Our interest in this research is in determining the feasibility of using GPS technology to acquire pedestrian travel data rather than in identifying the specific travel patterns of individuals. The identity of the subjects and the data collected from each subject will be kept confidential and will be known only to the researcher. The subjects will be given a number and any references to an individual subject will be made by their respective number.

\section{Getting Ready for the Experiment}

In order to successfully complete the GPS data-logger study, subjects will be expected to comply with the following:

- The GPS unit should be handled and operated carefully just as you would any electronic device.

- Each time it is worn, the antenna shall be placed correctly as demonstrated in the upright position on the shoulder.

- The subject should switch on the GPS unit for all walking trips. 
- The travel log form should be completed at the time of each motorized or nonmotorized trip.

\section{Experimental Procedure}

This experiment is being conducted to ascertain the feasibility of the wearable GPS data-logger for use as a pedestrian travel data collection device. You will be given the GPS unit at noon for a period of 24 hours. During this time, you should record all walking trips made out-of-doors. Whenever you leave a building to go for another destination by walking, you will need to switch on the GPS unit by connecting the wire from the battery pack to the data-logger, once you are outside. You will need to wait about 2 minutes at the start of each trip to allow the GPS device to determine your position, after which you can walk to your destination. Take care that the receiver/antenna of the GPS unit, which is present on the shoulder strap of the kit, is placed upright on one of the shoulders, as demonstrated. Once you have reached your destination, you can switch off the GPS unit by disconnecting the wire from the data logger. This process should be carried out for all walking trips during the specified period.

Along with the GPS unit, you will also be given a travel log form which should be completed for all trips you make (including other modes such as car, PRT and bus). You should clearly identify the origin/destination information in terms of a descriptive name for identification. Also note the time of departure/arrival for each location along with the mode of travel used. Again, the travel log form will be completed for all non-motorized and motorized trips, while the GPS unit will be switched on only for walking trips. 
At the end of specified 24 hours on the next day, return the GPS unit along with completed travel log form to the researcher. You will then be asked to complete a brief questionnaire on your experience. 


\section{GPS Travel Log Form}

\begin{tabular}{|c|c|}
\hline SUBJECT--- & DATE--- \\
\hline Origin- & Origin- \\
\hline Origin time - & Origin time - \\
\hline Mode- & Mode- \\
\hline Destination- & Destination- \\
\hline Destination time- & Destination time- \\
\hline Origin- & Origin- \\
\hline Origin time - & Origin time - \\
\hline Mode- & Mode- \\
\hline Destination- & Destination- \\
\hline Destination time- & Destination time- \\
\hline Origin- & Origin- \\
\hline Origin time - & Origin time - \\
\hline Mode- & Mode- \\
\hline Destination- & Destination- \\
\hline Destination time- & Destination time- \\
\hline Origin- & Origin- \\
\hline Origin time - & Origin time - \\
\hline Mode- & Mode- \\
\hline Destination- & Destination- \\
\hline Destination time- & Destination time- \\
\hline Origin- & Origin- \\
\hline Origin time - & Origin time - \\
\hline Mode- & Mode- \\
\hline Destination- & Destination- \\
\hline Destination time- & Destination time- \\
\hline
\end{tabular}




\section{Subject Post-Test Questionnaire on Using Wearable GPS for Collecting Non-Motorized Travel Data}

Subject

Date:

Date of Study:

1. Did you ever fail to take the GPS data logger with you any on any walking trips? If yes, about how many times?

2. Did you ever fail to switch on the GPS data logger? If yes, about how many times?

3. Did you ever forget to wait the two minutes needed for data logger start-up? If yes, about how many times?

4. Did you ever forget to take the travel log form with you when leaving a building? If yes, about how many times?

5. Did you ever fail to record information about a walking trip? If yes, about how many times?

6. Did you ever fail to record an entire trip on the travel data form? If yes, how many times?

7. Did you think it was uncomfortable/bothersome to carry the GPS data-logger everywhere for your trips outside?

8. Did you think it was bothersome to have to carry and record all trips made, on the travel log form?

9. Did the GPS unit receiver ever slip off your shoulders? If yes, would you say it happened - rarely occasionally frequently ? 
10. Did you feel that GPS data-logger affected your physical/personal appearance? How?

11. Did the GPS unit interfere with any movements or activities? If yes, please explain

12. Does it bother you that someone knows about all of the walking trips you made during the period of the experiment? 